\title{
Skirmishes on the border: \\ How children experienced, influenced and enacted the boundaries of curriculum in an early childhood education centre setting
}

by

\author{
Alison Margaret Stephenson \\ A thesis \\ submitted to the Victoria University of Wellington \\ in fulfillment of the \\ requirements for the degree of \\ Doctor of Philosophy \\ in Education
}

Victoria University of Wellington

2009 



\begin{abstract}
The notion of curriculum as contested was central to this thesis. In particular, the focus was on how children (aged from 8 months to 5 years) experienced and influenced the scope of curriculum and participated in the process in defining what constituted null curriculum in one New Zealand childcare centre. Qualitative methods were used to investigate this process of setting curriculum boundaries. Participant observations over five months yielded detailed observations, and these were supplemented by conversations with children which occurred in the context of a range of research strategies; children's perspectives have been foregrounded throughout. Data generation and analysis was guided by principles of the generic inductive qualitative model.
\end{abstract}

Critical pedagogy and the sociology of childhood together provided the theoretical and methodological framework for the study, and 'strategies of dislocation' were devised to assist in seeing unfamiliar aspects in a familiar context.

The central source of curriculum boundaries was found to be the assumed demarcation between adults and children; not only did this wider social norm influence the teachers, but it was also found to be embedded within the physical structure and organisation of the centre. It is argued this generational division conflicted with teachers' commitment to implementing sociocultural practices.

The core of curriculum for children was found to be relationships with others. However, many relationships were characterised by a dialectic tension between a desire to establish relationships and be accepted within the community, and a desire to exercise control/power. It is argued that these two concerns were significant aspects of curriculum for children. Children's focus on gender and their individual interests also influenced the scope of curriculum, although children's ability to introduce interests depended upon how conducive the physical and social environment was to their expression.

Teachers' and children's interpretation of what constituted null curriculum varied. Some aspects, and particularly the body, appeared to be null curriculum for all. Children used strategies of resistance to introduce new elements into the curriculum. 
Findings from the thesis are aligned with those of other recent qualitative studies in similar New Zealand settings and implications for the early childhood profession are discussed, particularly in relation to scrutinising the image of the child that is implied in practices, and challenging assumptions about the roles of adults and children, as a first step towards dismantling expectations that currently limit the potential scope of curriculum. 


\section{ACKNOWLEDGEMENTS}

I am grateful for the invaluable critique, unfailing support and personal encouragement offered by my supervisors Associate Professor Val Podmore and Professor Helen May. I also wish to acknowledge the interest and insights my friend Lynn Wright offered, and the value of a mid-way conversation with Dr Joce Nuttall. Colleagues on the Karori campus were a never-failing source of sustaining interest and empathy. But my deepest gratitude goes to the Centre community—children, teachers and families—who so generously allowed me to share their lives.

This project was made possible by the award of research leave from Victoria University, which allowed me periods for uninterrupted data-gathering, analysis, thinking and writing. Without this support, the thesis would not have been completed.

A special thank you to my partner Bill who was a tireless source of emotional and practical support and to my mother and sons who, from a distance, took an interest in the prolonged process.

This thesis is dedicated to the memory of my very dear friend Colleen Chandrahasen, whose living, laughing, loving and dying are, for me, inextricably woven through the words. 


\section{TABLE OF CONTENTS}

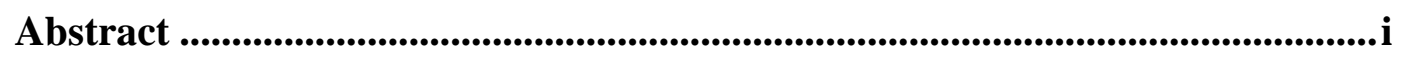

Acknowledgements ...............................................................................................................iii

Chapter 1 Introduction ......................................................................................................... 1

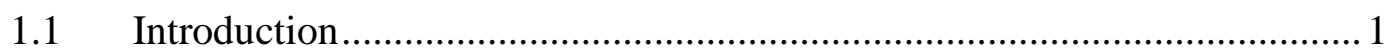

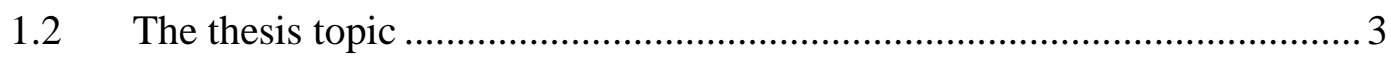

1.3 The personal journey to the research topic ...............................................

1.4 The professional and policy journey to the topic........................................

1.4.1 Changes in early childhood education in New Zealand since 1990 ......10

1.4.2 Including children's voices in assessment and evaluation.......................16

1.5 International strands of thinking and research within early childhood

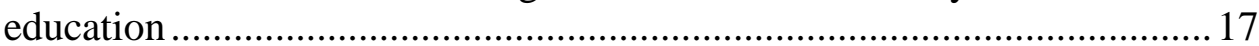

1.5.1 Reconceptualising early childhood education..........................................17

1.5.2 Including children's voices in research ....................................................20

1.6 The thesis aims, the research questions and the research setting ...............21

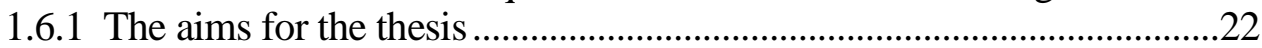

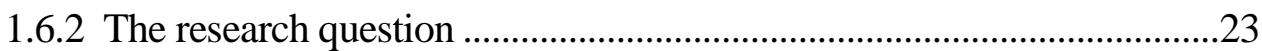

1.6.3 The research setting ……………………………………………….....24

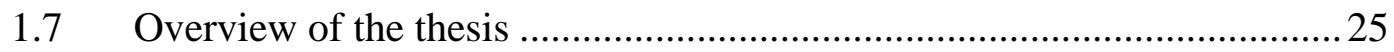

Chapter 2 The Theoretical Framework.....................................................................29

$2.1 \quad$ Introduction to critical pedagogy ………………….................................... 30

2.1.1 Critical pedagogy …………………………………………………......

2.1.2 Power 33

2.1.3 The hidden curriculum...............................................................................35

2.1.4 Defining the hidden curriculum ..............................................................36

2.1.5 The null curriculum..................................................................................

2.1.6 Defining the null curriculum ....................................................................40

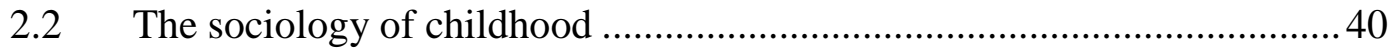

2.3 Activity theory as the source of sensitising concepts ..................................4 44

2.3.1 Introduction to activity theory .................................................................44

2.3.2 The elements from activity theory used as sensitising concepts.............46

2.3.3 Unit of analysis.....................................................................................4

2.4 The three strategies of dislocation ........................................................ 48

Chapter 3 Loose Ends and Longer Threads: The Literature Review ...........51

3.1 How might the physical environment influence the scope and boundaries

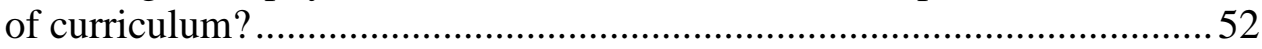

3.1.1 The physical and social isolation of centres .............................................52

3.1.2 The interior design and layout..................................................................54

3.1.3 The provision of resources ...................................................................56 
3.2 What content might be marginalised or considered null curriculum? .......59

3.2.1 'Real' tasks as curriculum? ...................................................................59

3.2.2 Are death and disaster appropriate curriculum content? ........................60

3.2.3 Are the body and sexuality appropriate curriculum content? ................61

3.2.4 Issues of social equity and justice as curriculum? ..................................62

3.2.5 Which interests are accepted as curriculum? ...........................................64

3.3 Which ways of thinking might be marginalised or considered null

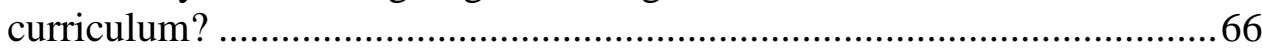

3.3.1 Do cultural assumptions influence the privileging/marginalising of ways of thinking?

3.3.2 Are there other ways of thinking that might be privileged/ marginalised?

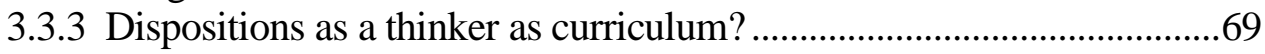

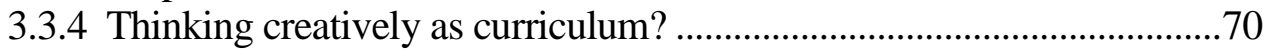

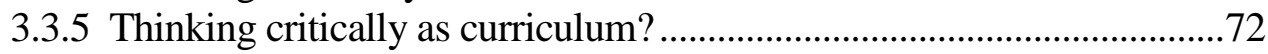

3.3.6 Children's schematic thinking as curriculum? ..........................................

3.4 What emotions might be marginalised or considered null curriculum? ....75

3.4.1 Teachers' emotional expression as curriculum? ........................................75

3.4.2 Children's emotional expression as curriculum? ....................................76

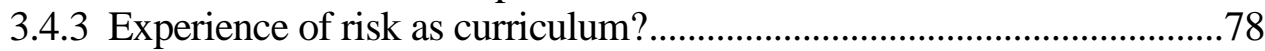

3.4.4 Religious and spiritual experiences as curriculum?...............................79

3.5 What is the role of the peer group in influencing and enacting

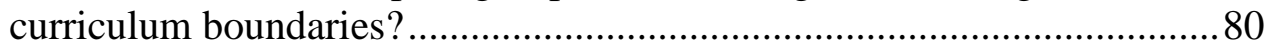

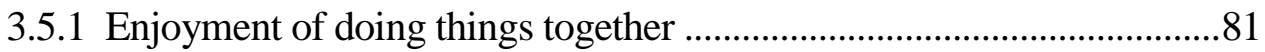

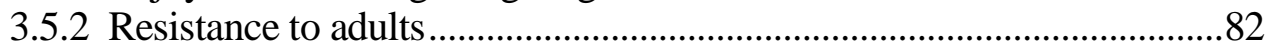

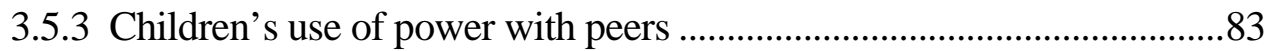

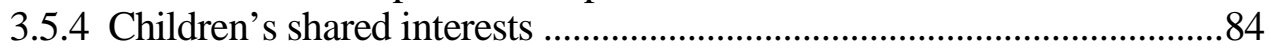

3.5.5 Gender 85

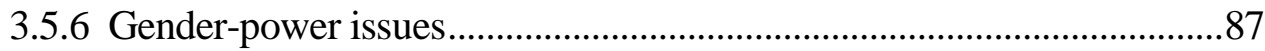

Chapter 4 Methodology

4.1 The route from constructivist grounded theory to the generic inductive

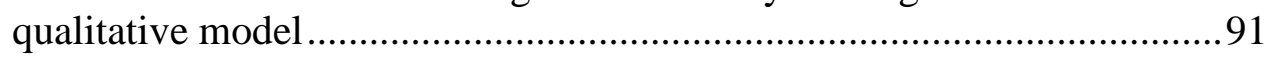

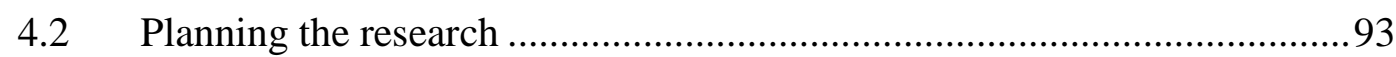

4.2.1 Using ethnographic methods............................................................93

4.2.2 Identifying potential strategies to foreground children's perspectives

4.2.3 The six focus children....................................................................100

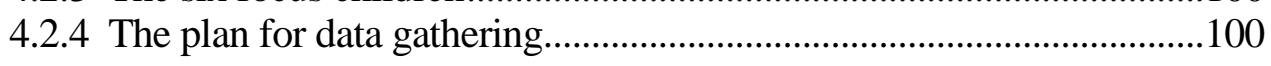

4.3 The human dimension ........................................................................ 102

4.3.1 Locating and gaining access to a centre ..............................................102

4.3.2 Establishing and maintaining ethical relationships with children.........104

4.3.3 Establishing and maintaining a research relationship with the adults .107

4.3.4 Establishing and maintaining a research relationship with the families 108

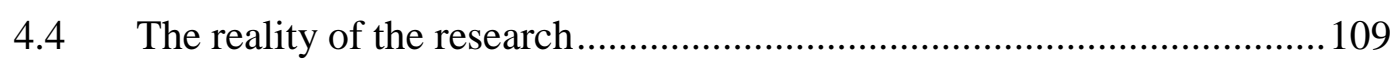

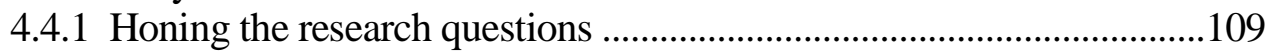

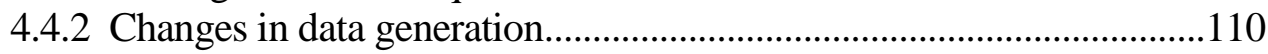

4.4.3 The development of child-focused strategies.........................................111

4.5 The management and analysis of the data............................................... 114 


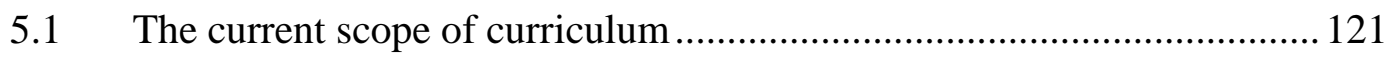

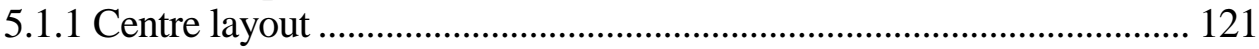

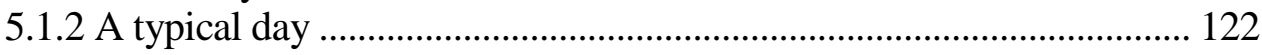

5.1.3 Planning documentation for a typical six-week period ....................... 126

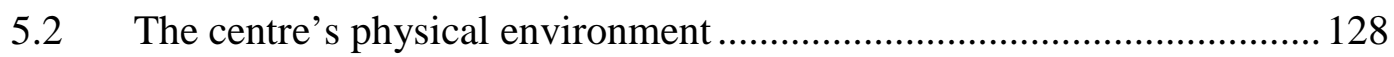

5.2.1 Implications of the adult-child demarcation embedded in the centre's location ................................................................................. 128

5.2.2 Implications of adult-child demarcation embedded in the centre's architecture ............................................................................ 130

5.2.3 Implications of the adult-child demarcation embedded in the

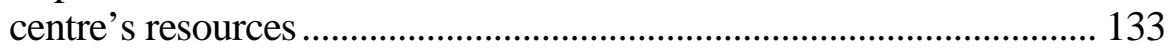

5.2.4 Children's activities: Further evidence of the adult-child demarcation ................................................................................... 140

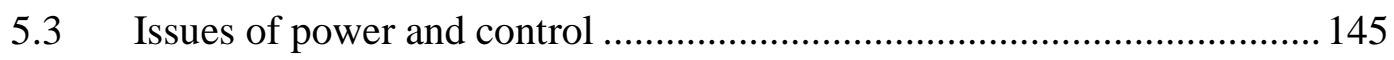

5.3.1 The centre's architecture: Implications of power/control.................... 145

5.3.2 The windows: Implications of power/control .................................... 150

5.3.3 Teachers' control of resources ............................................................. 152

5.3.4 Children's resistance to teachers' control of equipment.......................... 155

5.4 The importance of relationships and community.................................. 159

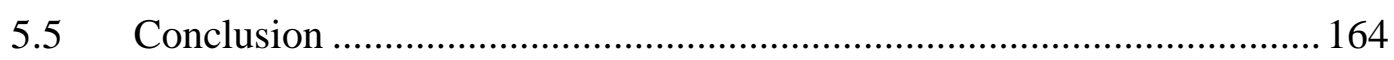

Chapter 6 How teachers' and Children's Roles Influenced the Boundaries of Curriculum ....................................................................167

6.1 Teachers' roles from the children's perspective ..................................... 168

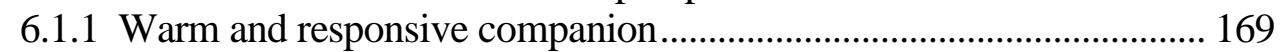

6.1.2 Day-to-day carer who looks after my physical needs and wants........ 171

6.1.3 Initiator/organiser/controller of resources, activities and events......... 171

6.1.4 Monitor of the social and emotional climate......................................... 171

6.1.5 Documenter of children's learning ...................................................... 172

6.2 The second emergent theme-participation in the community and the exercise of control/power as curriculum................................................ 175

6.2.1 Relationships and living within a community as the core of

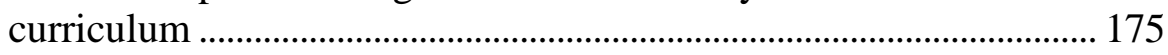

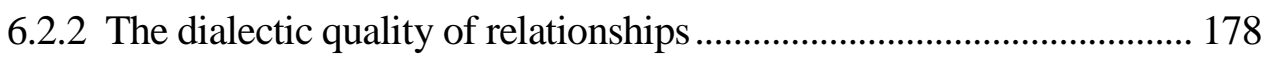

6.2.3 Relationships with others and control/power as curriculum

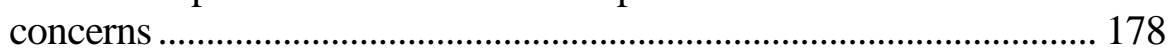

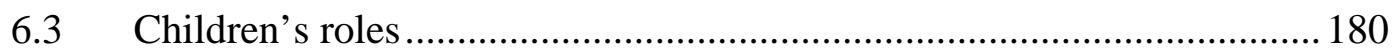

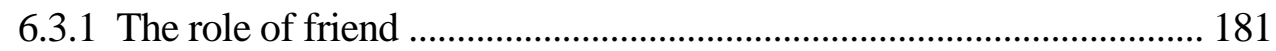

6.3.2 Gender-defined roles................................................................. 187

6.3.3 Children as peer teachers/learners........................................................ 194

6.3.4 Do children see themselves as learners?............................................... 198

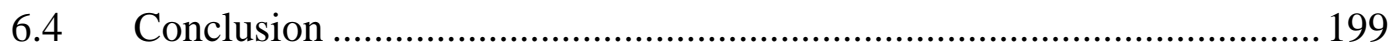




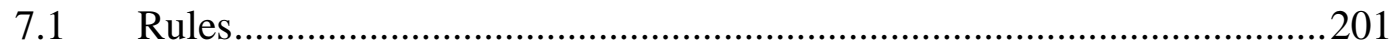

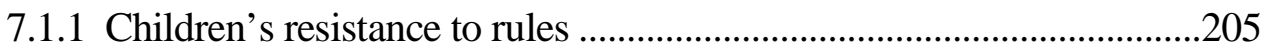

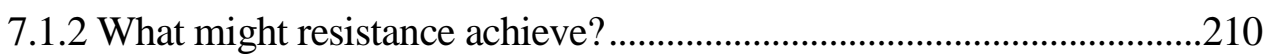

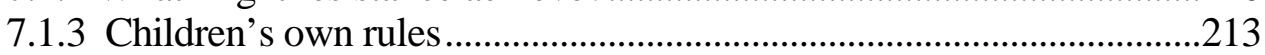

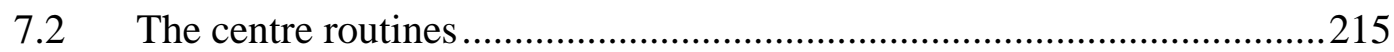

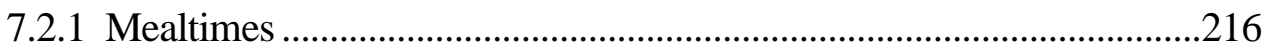

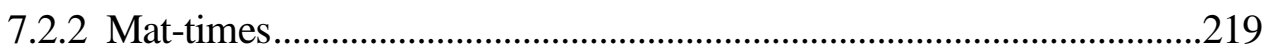

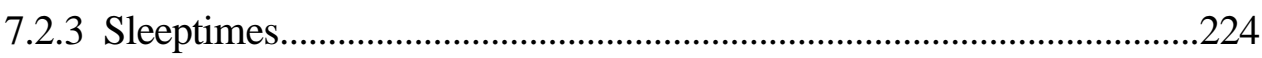

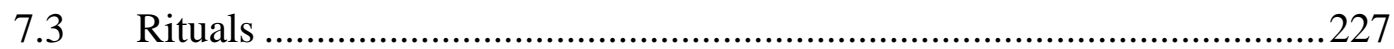

7.3.1 The fifth birthday ritual...................................................................22

Chapter 8 The Null Curriculum......................................................................2231

8.1 What was null curriculum for children?...........................................233

8.1.1 What was null curriculum for all/most children? ..............................233

8.1.2 What was null curriculum for some children? ...................................236

8.2 What children considered to be null curriculum for teachers .................238

8.3 What was null curriculum for teachers .............................................. 240

8.3.1 What was not null curriculum for teachers ...........................................240

8.3.2 Content that was null curriculum for teachers ....................................241

8.3.3 Intellectual processes that were null curriculum for teachers ..............247

8.3.4 Emotional dimensions that were null curriculum for teachers.............254

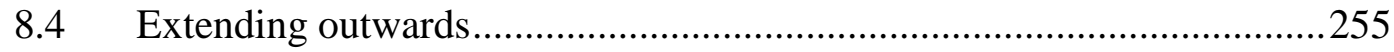

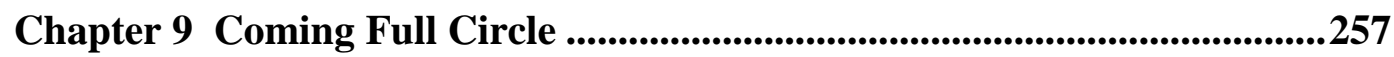

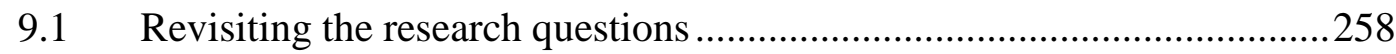

9.1.1 The adult-child demarcation as a source of curriculum boundaries ....259

9.1.2 How did the learning and teaching among children affect the

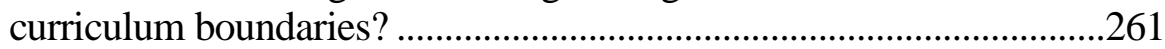

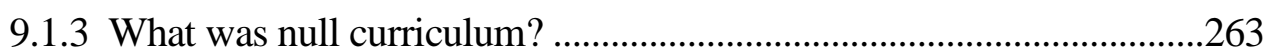

9.1.4 What are the boundaries of curriculum, and how do young children experience, influence and enact these? ....................................264

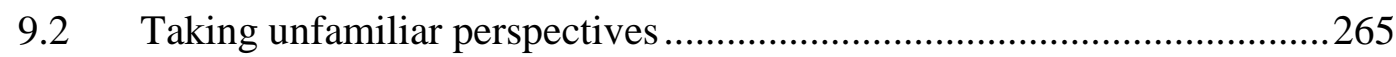

9.2.1 The theoretical framework ..............................................................265

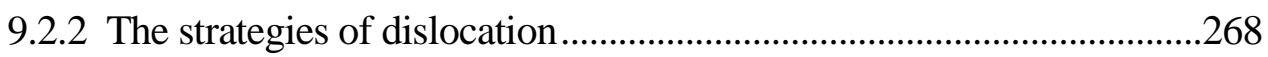

9.2.3 The focus on children's voices ............................................................268

9. 3 In the context of other recent New Zealand theses ...............................2276

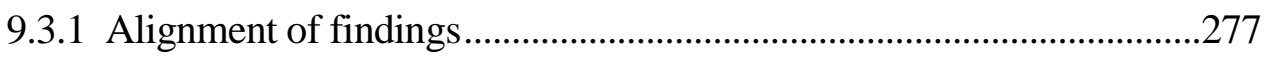

9.3.2 Alignment of methodological features .............................................281

9.4 Implications for the early childhood education profession ......................2282

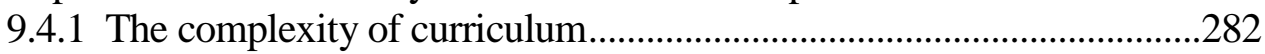

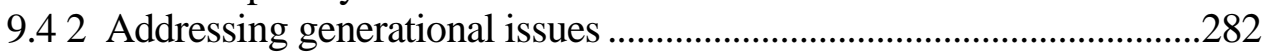

9.4.3 Recognising the complexity of children's social lives .........................287

9.4.4 Adopting an attitude of openness.........................................................28

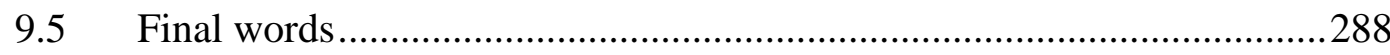


Appendices...

Appendix 1: Information sheet for centre management, teachers and families .. 326

Appendix 2: Permission letter to Centre Management....................................... 328

Appendix 3: Consent form for Centre Management .......................................... 331

Appendix 4: Consent form for adults in the Centre........................................... 333

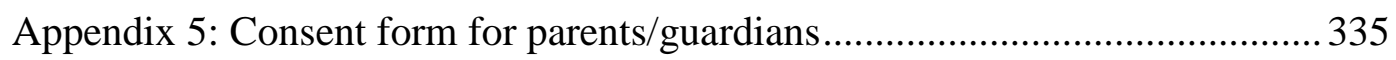

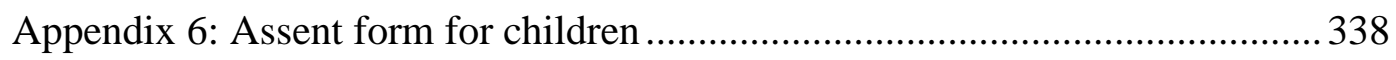

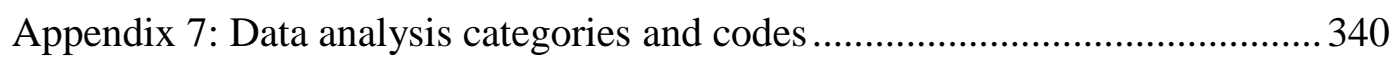

\section{Table}

Table 1: $\quad$ Planned stages of data generation 101

\section{List of Figures}

Figure 1: The three dimensions of enacted curriculum ................................... 2

Figure 2. The structure of a human activity system.......................................... 46

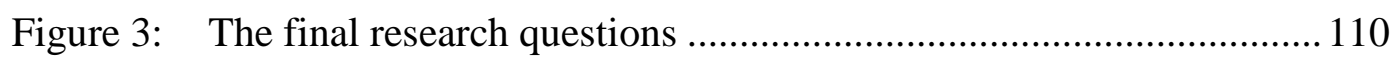

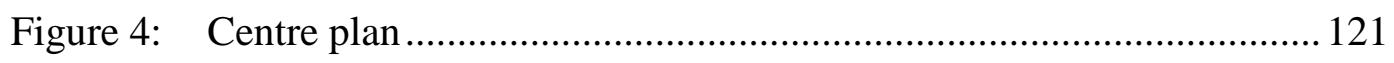

Figure 5: Adult spaces and communal spaces in the centre ............................ 131

Figure 6: Teachers' roles, as children experienced them ................................ 169

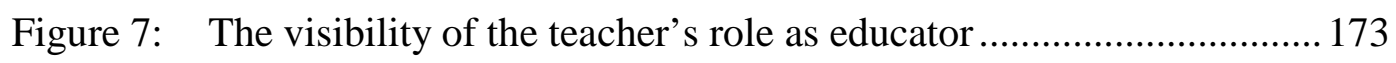

Figure 8: The roles implied for children by the teachers' roles ....................... 174

Figure 9: Factors contributing to variation in how children experienced

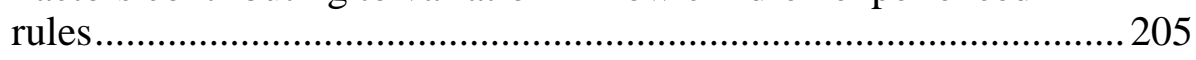

Figure 10: The differing boundaries of null curriculum for children and

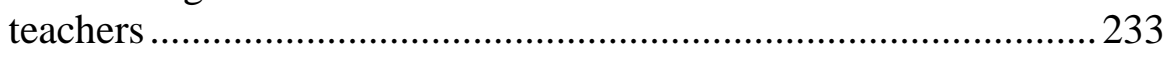




\section{CHAPTER 1}

\section{INTRODUCTION}

All my life I have fought against formula. Once you have set down a formula, you are imprisoned by the witch doctor's magic circle. (Pratt, 1948/1990, cited in Goffin \& Wilson, 2001, p.227)

\subsection{Introduction}

My elderly dictionary, published in the 1960s, defines curriculum as "a regular course of study" suggesting it is an unproblematic concept. Scholarly writing (e.g., Pinar, Reynolds, Slattery \& Taubman, 1995) indicates more careful interrogation is required and shows a much wider range of meanings has been attached to the word since that dictionary entry was published. There has been since then a period of "curricular reconceptualisation' (Pinar et al., 1995) during which ideas from critical (e.g., Giroux, 2001; McLaren, 1998), feminist and postmodern (e.g., Lather, 1991) discourses have been drawn on to challenge established conceptions of curriculum. Challenge has come both from recognition of curriculum's role in conveying and reinforcing the hegemonic patterns of society (e.g., Apple, 2004; Giroux, 1997; McLaren, 1998) and as part of the wider critique of positivism with a rejection of the notion of curriculum as a defined body of knowledge. Aligned with this, curriculum is increasingly understood as the lived reality, reflected in descriptions such as "actual lived situations" (Pinar et al., 1995, p. 424) and "it is about life itself" (Hill, 2005, p. 26). Reviewing the concept of curriculum from the first decade of the 21 st century, it seems much of the certainty implied in that dictionary definition is under threat. This thesis is written within the context of these emerging understandings.

In particular, the thesis draws on the notion of curriculum as contested, as "the site on which the generations struggle to define themselves and the world" (Pinar et al., 1995, p.848), as the place where "dominant and subordinate voices define and constrain each other, in battle and exchange, in response to the socio-cultural conditions "carried" in the institutional, textual, and lived practices" (Giroux, 1997, p.133). It will explore the enactment of curriculum in a single site-an early childhood education centre in New Zealand-and alongside the images of cohesive community, it will be shown there were elements of 'struggle' in the children's responses to limits that teachers, consciously or unconsciously, imposed on curriculum. 
An image of two concentric circles superimposed on a wider landscape (see

Figure 1) represents my conception of curriculum within the centre. The inner circle represents the direct or overt curriculum, the acknowledged and intended teaching and learning that occur, familiar territory to me as a teacher educator and a past teacher of young children. The area enclosed between the boundaries of the inner and the outer circles represents the unintended, unplanned and often unacknowledged aspects of curriculum, the indirect or covert curriculum. The outermost border represents the line of demarcation between what is deemed relevant/irrelevant, appropriate/inappropriate and so constitutes the boundaries of curriculum in the setting. The null curriculum (Eisner, 1985), the content or aspects that are, consciously or unconsciously, excluded, is the area beyond the circles. As this thesis will show, some points along that outer boundary are debated, but others are accepted unquestioningly.

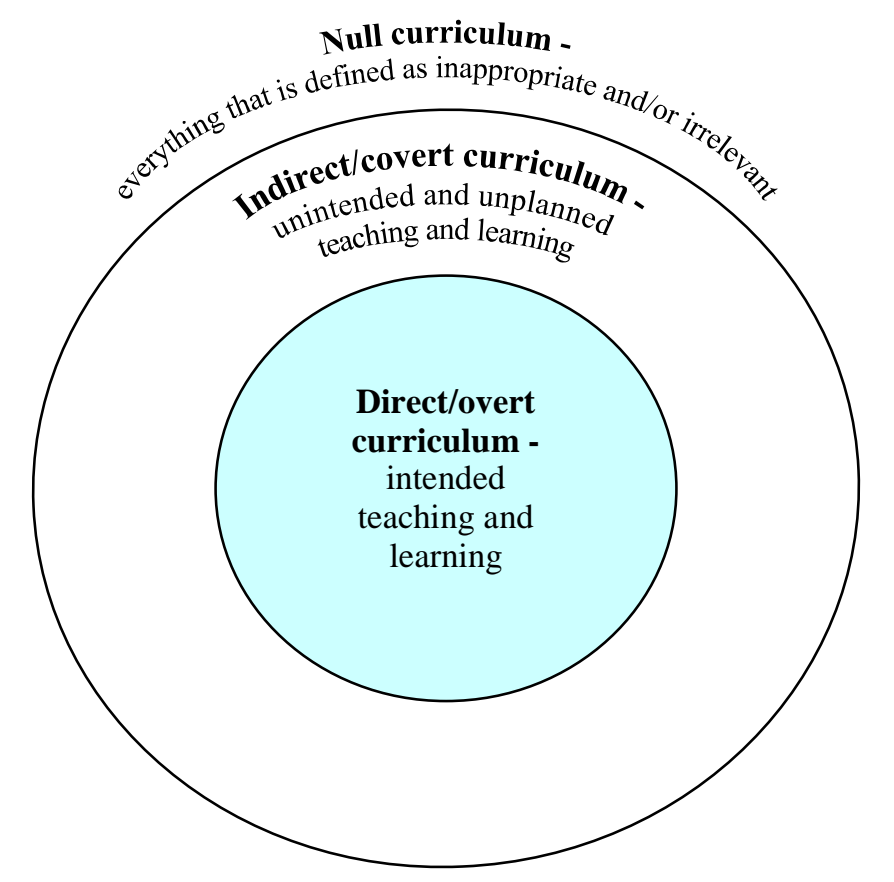

Figure 1: The three dimensions of enacted curriculum

This thesis journey began with the broad intention of exploring the full scope and boundaries of the second circle in the early childhood centre setting. Or, in other words, the initial focus was on exploring the covert curriculum, all that was unintended/unplanned and identifying the boundaries that were placed on curriculum. Later, and in keeping with principles of the generic inductive qualitative model (Hood, 2007), the thesis topic narrowed to an exploration of the outer border, the boundary between what was included/excluded with a particular focus on how children experienced, enacted and influenced that boundary. 'The 
centre' is the pseudonym for the early childhood education centre where I spent five months observing children's and teachers' lives together, and talking with children. Because a commitment was made to foreground children's perspectives throughout the thesis, teachers were not interviewed. That term, children's perspectives, has been used here to cover two elements (following Strandell, 1997, cited in Broström, 2006). The first is 'children's knowledge' (Mayall, 2000) which involves listening to, and hearing, what children say. The second involves 'taking the perspective of children' through a process of observing and interacting with them, and on that basis imagining and identifying with how they think and feel. (The methodology is described in detail in Chapter 4.)

In this chapter the thesis topic is introduced, and the route to its selection described. After acknowledging personal factors which influenced the choice, there is a review of the strands of thinking and research within the discipline of early childhood education, both nationally and internationally, that were influential in developing the topic. The research aims, research questions and the centre that participated in the research are introduced and the chapter concludes with an overview of the thesis.

\subsection{The thesis topic}

The thesis topic is grounded in the definition of curriculum used in the New Zealand curriculum guidelines for early childhood, Te whāriki: Early childhood curriculum. He whāriki mātauranga mō ngā mokopuna o Aotearoa: Early childhood curriculum (Ministry of Education, 1996). This document first appeared in draft form in 1993 (Ministry of Education, 1993b), and since 1996 has been the guiding curriculum document for all early childhood education contexts in New Zealand. Here "curriculum" is defined as:

the sum total of the experiences, activities, and events, whether direct or indirect, which occur within an environment designed to foster children's learning and development. (Ministry of Education, 1996, p.10)

There was a reference in the draft of Te whäriki (Ministry of Education, 1993b) to the 'hidden curriculum', explained as “the often unplanned experiences that arise from where and how both adults and children spend their time, the kinds of interaction between them, assessment procedures, and the physical characteristics of the environment" (p.13). While this was removed from the final version as part of the Ministry of Education's revision process, the current definition is 
nevertheless striking for its acknowledgment that the scope of what children learn extends beyond the scope of what teachers intend to teach, particularly when compared with the curriculum document for New Zealand schools, the New Zealand Curriculum Framework (Ministry of Education, 1993a) that appeared as part of the same educational reform process. A New Zealand scholar and early childhood education professional, Haggerty $(1998,2003)$, recognised that the breadth of the Te whâriki definition and its emphasis on the reality of what children learn, aligned it with the reconceptualist views of curriculum referred to in the opening paragraphs. In identifying this, Haggerty was making an early contribution to what was to develop into an ongoing conversation between New Zealand researchers, a conversation which continues to provide insight into the ways in which the new curriculum has been implemented.

Te whăriki was also striking for its lack of definition about what constitutes the contents of that "sum total". It offered a framework of principles, aims (later renamed strands) and goals which each setting could 'weave' to suit their unique context. In contrast, the new school curriculum prescribed learning principles, aims and objectives (Mutch, 2003). However, the broad scope of the definition in Te whăriki was not unique within early childhood education; internationally there were similar examples. For example, in the United Kingdom Curtis (1998) offered the following definition:

Curriculum is everything that affects the child in the learning environment, overt and covert. It covers not only the activities, both indoors and outdoors, offered to young children, but the attitudes of the staff not only towards the children but to each other, to parents and anyone else who visits. (p.21)

While such understandings of curriculum in early childhood education are not universal (MacNaughton, 2003b), the definitions above reflect a growing acceptance of the notion that curriculum for young children is an allencompassing concept including intended and unintended aspects. There is, however, a danger in using such binaries as intended/unintended and overt/covert, just as there is in using that ordered opening image of circles to depict the divisions between overt, covert and null. Such categorisations provide useful tools of analysis, but can mask the complexity of the multi-faceted, multi-layered and multi-tensioned reality of the shared lives of the children and teachers. While this thesis makes use of such categories as tools, they are used with the intent of revealing rather than masking such complexity. 
Within New Zealand research direct aspects of curriculum, the teaching/learning activities that teachers consciously engage in with children (the familiar territory of the inner circle), have frequently been the focus (e.g., Jordan, 2003; Meade, 2005, 2006, 2007; Smith, Duncan \& Marshall, 2005). Recently, however, indirect aspects of curriculum have increasingly drawn research attention, reflecting the broader notion of curriculum in Te whäriki. For example, Carr (1997a) showed the impact that children's self-selected identities such as 'being a technologist', 'being nearly five', 'being a friend' can have on their approach to experiences in the centre. Duncan's (2004) description of the impact of wider discourses on children's approach to learning and Ritchie's (2001) discussion of the impact of political ideology on practices within early childhood education are further examples. But references to the concepts of the 'hidden curriculum' (Hill, 2001, 2005; Nuttall, 2002; Podmore, Sauvao \& Mapa, 2003) and 'null curriculum' (Hedges \& Cullen, 2005) are rare. Little research or academic writing has considered the borders of curriculum or the territory beyond, the null curriculum. Among the few examples are Duncan $(1998,1999)$ and Tait $(2001)$ who discussed the effect of highly publicised sexual abuse cases on teachers' attitudes and relationships with children; and Surtees (2003) who described how exclusion of sexuality and the positioning of heterosexuality as normality leads to the exclusion of other sexual orientations. Others touch in passing on what is excluded; Hedges and Cullen (2005) suggest subject knowledge may be null curriculum, and Sanson (2006, 2007), writing about dance, describes the body as increasingly marginalised.

Another recent and growing trend within New Zealand early childhood research is the inclusion of children as participants. Smith was an early proponent, interviewing children in childcare in the 1980s (Smith \& Swain, 1988) and others (e.g., Smith et al., 2005; Te One, 2007) have experimented with a wider range of approaches since.

It is timely to introduce into this discussion the recent surge of New Zealand masters and doctoral theses that have used qualitative methods and focused, in a range of ways, on aspects of curriculum implementation in early childhood settings. Collectively, these form the conversation referred to earlier. Some of these studies reflect the two trends referred to above- the focus on indirect aspects of curriculum, and the inclusion of children as participants. For example, 
children's perspectives have been to the fore in the studies by Greenfield (2007), Brennan (2005), Alcock (2005) and Te One (in preparation), and were also included by Brennan (1999) and Hedges (2002b, 2007). Drawing the line between those theses that focus on indirect and/or direct aspects of curriculum is not always easy, given most have been broad-ranging qualitative studies, but indirect aspects of curriculum were to the fore in Brennan's (1999) study of the influence of television on children's learning, and her more recent work (2005) on children's enculturation into a centre, in Dalli's (1999) study of very young children's transition into a centre, and in Alcock's (2005) investigation of children's humour. Among those studies where more direct aspects of curriculum have been to the fore are Wright's (2003) description of curriculum in a homebased setting, Hedges' (2002b) study of the place of subject content knowledge, and her more recent (2007) exploration of the interplay between teachers' and children's funds of knowledge. Some have explored topics where teachers are the focus: Jordan (2003) and Bernstone (2007) both considered teachers' use of strategies, Nuttall (2004) explored teachers' understanding of curriculum, and Manning (2008) described how teachers' earlier careers were reflected in their practices. Finally, two studies (Gibbons, 2004; McLeod, 2002) highlight the link that exists between the construction of the child and the management practices and organisational culture of the centre. Despite the diversity in topics and approaches, there is a sense of growing interplay as later writers revisit and respond to earlier work; collectively they provide an unprecedented depth of insight into current New Zealand practices. There is also an increasing confidence to critique and question established traditions, and there is growing awareness of the role that social forces, beyond the arena of early childhood education, may play in defining what constitutes appropriate curriculum for young children. In acknowledgement of the value of this emerging collective conversation, links to this group of theses have been profiled throughout, and in the final chapter findings from this thesis are aligned with findings from others in this group.

Reviewing recent research therefore indicated gaps where this thesis might contribute. First, there was growing interest in indirect aspects of curriculum, and accumulating evidence about how these might be influencing teaching and learning. However, the distinction between what was deemed irrelevant/relevant and how that demarcation was arrived at had rarely been considered. Secondly, there was increasing interest in hearing children's thinking. It seemed therefore 
that taking a focus on how children experienced and influenced the boundaries of curriculum might provide insight into where those boundaries lay, describe how they were established and reinforced, and what part children might play in that process. It was hoped this would contribute to accumulating research knowledge about curriculum implementation in New Zealand centres, and would also provide further insight into children's thoughts and feelings about their day-to-day experiences of centre life.

In the next section, I trace the combination of factors, both personal and professional, that led me to select this topic.

\subsection{The personal journey to the research topic}

Clarifying my position in relation to the research is an important element of validity because as the sole researcher I was integral in the processes of data generation and analysis (Denscombe, 2007). Explicitly acknowledging those personal factors and experiences is a first step in that process. Some relate to my own experiences of learning and teaching, some reflect my interest in children's perspectives, and some are associated with the quality of the question itself.

I have a long history of interest in the broader reaches of learning and teaching that stems partly from belonging to a family of teachers and self-motivated learners, and partly from having worked in early childhood, primary and tertiary education. I have a particular commitment to the significance of the social context in learning/teaching which is rooted in my experiences in early childhood education with my two sons. When they were young, I gravitated to my local playcentre. These parent-led cooperatives are a significant feature of the New Zealand early childhood education scene; alongside care and education for children, they offer ongoing training for parents, and require parent commitment during sessions. The movement began during the Second World War, grew quickly, and became a source of innovation within early childhood education, particularly through the next two decades (Stover, 2003). In taking my children there, I was confident they would be learning and felt comfortable with the commitment to free play and the strongly Piagetian approach that pervaded New Zealand early childhood education at that time. With a Masters degree, and four years of primary school teaching behind me, it never occurred to me that I too might be embarking on a learning journey. While I have later questioned some of what I learnt in playcentre, much of which was embedded in a developmental framework 
(e.g., Grey, 1974), the impact of how I acquired that learning has stayed with me. To find myself in the social contexts in which teaching/learning occurs within playcentre was a humbling awakening to the potential of learning in group contexts, of the multitude of ways in which teaching/learning can occur, and of how satisfying these learning journeys can be.

My experience as a primary school teacher threw into sharp relief the openendedness and lack of content specification in the Te whäriki definition of curriculum, and the consequent freedom, challenge and responsibility that implied for teachers. As a lecturer, I revisit that definition with students every year and each year I pause at the word "indirect" and reflect on how much of our teacher education programme focuses on the "direct" experiences, activities, and events that teachers provide. I recognise how infrequently we question the choices of resources and experiences that are deemed to be appropriate provision for young children. Blocks and playdough, puzzles and dress-up clothes, drawing and collage, stories and singing on the mat, and outside a sandpit, slide, bikes and swings. These were the experiences that were provided for my sons, that I remember from my own childhood, and these are the typical experiences in centres today. Comparing the current basic equipment guidelines for centres (Ministry of Education, 2004a) with the basic equipment list for playcentres 40 years earlier (Somerset, 1967) shows remarkably few changes. These experiences made me curious about the origins of some of the rarely questioned conventions of early childhood education, and heightened my awareness of the tension between the broadness of the definition of curriculum in Te whäriki and the parameters that, in practice, seemed so often to be imposed.

A second personal impetus in selecting the topic was that I am drawn to the challenge of conveying how the world is for children. This is partly a commitment to equity, to allowing the voices of a usually silenced group (Cannella, 1997) to be heard, but the element of personal satisfaction needs to be acknowledged. Previous use of ethnographic research methods (Stephenson, 1998) with young children had been a rewarding experience that offered unexpected insight into the rich world of children's centre lives. A further incentive was that I was aware of only a handful of researchers (e.g., Corsaro, 1985, 2003; Hadley \& Nenga, 2004; Mandell, 1991; McCadden, 1998; Paley, 1986, 1990, 1995), who had spent a prolonged time in a research setting with young children. 
Finally, I recognise being drawn to topics that challenge institutional assumptions, to topics that are on the periphery of our understanding, unable to be settled by a single answer, but rather need to be opened up in order to provoke further discussion and debate. Perhaps because I have a previous academic background in sociology and English literature, and had a series of careers before finding early childhood education, I am drawn to topics that ask to be looked at from a range of perspectives. This personal background and these experiences led me to a commitment to research that focused on children's experiences and to an interest in the scope and boundaries of curriculum.

These varied threads were drawn together in thinking provoked by reading the following sentences in Carpenter (2001):

The overt or the intended curriculum is the publicly advertised fare of schools. But the learning gained in educational environments is not necessarily planned for. Unplanned-for learning makes up the 'undocumented' or 'hidden' curriculum, while some subject content that is deliberately excluded is called the 'null' curriculum. (pp.110-111)

Linking the concept of 'hidden' with the indirect curriculum, I saw that the concepts of hidden and null curricula were tools that could be used in a study of curriculum in a way which drew together my interest in the wider reaches of learning/teaching, my commitment to foreground children's perspectives, and my penchant for complex topics. The hidden and null curricula will be discussed in detail in Chapter 2.

\subsection{The professional and policy journey to the topic}

The personal journey to the topic outlined above was mirrored by a professional and policy journey, which is described in this section. Part of that journey was the process of finding the theoretical framework that would underpin the thesis; this is introduced below. The other dimension of the journey, seen clearly only in hindsight, was the way in which my thinking had been challenged by national and international developments within early childhood education.

There are two broad approaches, both of which extend beyond the field of early childhood education, that in combination informed the development of the topic, influenced the methodological design for this thesis, and provided a theoretical context. The first to have an impact was critical pedagogy. Reflecting on my role as teacher educator had led me into this area and it was within this discourse that the 
research topic and questions were framed, and it provided the theoretical framework for the thesis. As my interest in the topic grew, locating and reading research in which children's perspectives had been included led me into a very different area of research literature, that of sociology. I discovered that familiar current features of early childhood research, such as the focus on including children as actors in their own right and awareness of the concomitant ethical concerns, were reflections of a wider research arena, the sociology of childhood, which had arisen in the late 1980s (Prout \& James, 1990). The perspectives of the sociology of childhood provided a philosophical foundation for the study of children, and research ideas from this arena were valuable in designing the methodology (Clark, 2004). Finally, there was a smaller contribution from a third theoretical approach. Elements from activity theory have been used as sensitising concepts (Bowen, 2006); the constructs of the physical tools and resources, the roles of children and teachers, and the rules, routines and rituals of the setting were used as foci within data gathering and analysis. Chapter 2 presents a more detailed discussion of critical pedagogy and the sociology of childhood, outlining the reasons for their selection, and describes the individual contribution each made to the framing and implementation of the research. Activity theory is introduced in order to explain the way in which the three concepts borrowed from it were used within the thesis.

The second dimension to my professional journey to the topic relates to the professional and policy issues that have been part of the milieu of early childhood education in New Zealand through the last decade of the $20^{\text {th }}$ century, and the beginning of the $21^{\text {st }}$. The topic is located at the intersection of two current areas of focus: the questioning of what constitutes curriculum for young children and the reconsideration of how children are positioned. These are significant areas of debate nationally and internationally, which have disturbed the equilibrium of early childhood education.

\subsubsection{Changes in early childhood education in New Zealand since 1990}

This section describes how changing circumstances within early childhood education in New Zealand since 1990, largely engendered by the introduction of the new curriculum Te whâriki, have brought significant changes to the definition of what constitutes curriculum, and to conceptions of teaching and learning. As a teacher educator I experienced the hurdle of working through these changing theoretical orientations and was aware of the challenge they presented for teachers. 
In the following discussion, and through the thesis, the term early childhood education is used to denote the education and care provision for children aged from birth to 6 years (although most New Zealand children begin school when they turn 5). The term 'centre' is used generically to describe any setting that provides care and education for groups of children. 'Service' is used to refer to the different kinds of education and care centres: kindergartens (which provide Statefunded, sessional education and care typically for children aged from 3 to 6 years), education and care centres (which are either privately owned and operated or community-based, and which provide full-day care and education for children from 0 to 6 years), the parent co-operative playcentres (which provide sessional care for children 0-6 and training courses for parents), centres with a particular philosophical orientation (e.g., Steiner, Montessori), kohanga reo (Maori-language immersion centres), and Pacific Island language early childhood centres. (All these services receive a level of government funding.) 'Sector' is the collective used to describe all these varied services and individual centres, and 'the profession' refers to all early childhood education teachers and teacher educators.

The period since 1990 has been a tumultuous one for New Zealand early childhood teachers, with long-standing beliefs about curriculum and pedagogical practices fundamentally challenged. At the end of the 1980s, the early childhood sector was fragmented and "diversity reigned on all fronts: there was diversity of programmes, diversity of staff training and qualifications, and diversity of history and philosophy" (Dalli \& Te One, 2002, p.178). The idea that there could be a sector-wide curriculum was foreign. Rather, each service had its own philosophical and pedagogical approach which provided the programme guidelines and so determined the curriculum. In playcentre it centred on providing 16 basic play areas; the influence of these had flowed over into kindergartens, where play contexts such as the family corner and the carpentry table had become "the 'subjects' of preschool” (Middleton \& May, 1997, p.261). Students in teacher education programmes learned about 'play corners' and stages of play (May, 2001) as well as covering more traditional school-type content such as early mathematics and early music. However, the word 'curriculum' was rarely used and when it began to appear at the beginning of the 1990s it was seen as "threatening" (Nuttall, 2003, p.8), with its overtones of the compulsory school sector which had long operated with various national syllabuses, standards and curriculum documents. 
But at a political level there was a commitment to develop curriculum guidelines that would apply to all early childhood education contexts (Te One, 2003), and the first moves had already been taken. At a Department of Education policy development course in 1988, which brought together representatives from various services, a definition of curriculum had been honed that was to provide the genesis for the Te whâriki definition: "The curriculum is the sum total of all children's direct and indirect learning experiences in early childhood settings" (O'Rourke, 1988, p.17). In September 1990, in response to a national curriculum document for schools, the formal process of developing the new national curriculum began with the request for proposals. The contract was signed with Helen May and Margaret Carr from Waikato University, and a process of consultation and writing began that led to the release of the draft curriculum guidelines in 1993, and the revised final document in 1996.

When it was introduced, for many teachers the significance of Te whäriki was only as yet another document that needed to be understood and implemented, although extensive government investment in supporting professional development eased this. With hindsight its significance has become much clearer. It was the first genuinely bicultural New Zealand curriculum document, containing parallel but not identical versions in Māori and English. It consolidated the combination of care and education in a way that was recognised as probably unique in the western world (Cullen, 1996). It was one of the first two early childhood curricula internationally to include infants and toddlers (Haggerty, 2003). The widespread and lengthy consultation undertaken in its development, the supporting cross-service professional development, and the introduction of a shared common language of principles, strands and goals all helped to bridge long-standing divisions in the sector. Politically it was significant because, despite being initiated in a "neo-liberal/neo-conservative" political climate with government commitment to intervention in education, it managed to "maintain its liberal-progressive/socially critical discourse" (Mutch, 2003, p.123). Finally, Te whāriki was significant for the status it brought to early childhood education. Within New Zealand the curriculum was recognition of the importance of young children's learning and so, by implication, it brought recognition for their teachers. Internationally $T e$ whāriki was acknowledged for its "enormous impact on curriculum development in many countries" (Fleer, 2003b, p.243) and increasingly cited (e.g., Drummond, 2000; MacNaughton, 2003b; Wood \& 
Attfield, 2005). It was selected for showcasing as one of five early childhood curricula by the Organisation for Economic Cooperation and Development (Pramling Samuelsson, Sheridan \& Williams, 2006). However, when Te whāriki was introduced, little of this significance was recognised.

The introduction of Te whäriki, and the associated professional development, provided a catalyst for teachers to review and revise ideas about what constituted curriculum, even within that first familiar circle of teachers' planned teaching. For many this did not happen quickly. There were two reasons why teachers were slow to engage with the document's inherent challenges. First, anxiety that a curriculum would shunt the sector towards subject-focused teaching (Carr \& May, 2000) was allayed by the draft guidelines, which perhaps lulled teachers into believing the document merely captured the essence of what they were already doing.

The second reason teachers were slow to recognise the depth of change relates to Te whäriki's non-prescriptive approach. From the beginning there had been a commitment to cater to the sector's diversity as reflected in the wide-ranging consultation (Te One, 2003), in the document's bicultural and bilingual approach, and in the open-ended structure which required centres and services to 'weave' their own curriculum to suit their own context. But this open-endedness, which was a strength and a powerful force for cohesion in the sector (Cullen, 2003a, p.163), also masked the embedded shift and made it possible for teachers to engage with the curriculum by using its language to justify their unchanged practices.

The philosophical underpinnings of the new curriculum did imply a fundamental shift (Haggerty, 2003); they implied a move from an accustomed Piagetian and developmental orientation to a sociocultural approach and to a commitment to learning as socially constructed, and supported and extended through social interactions with peers and adults (Cullen, 1996). However, the inclusion of familiar developmental aspects, such as the divisions into age groupings (Cullen, 1996; Hedges, 2003) and the metaphor of 'acquiring' knowledge (Hedges, 2007), provided a sense of familiarity that potentially contributed to teachers' confusion about the implications for their role. As early as 1996, concern was expressed about the intertwining of the sociocultural and developmental perspectives and 
Cullen (1996) questioned whether teachers and teacher educators had sufficient knowledge to convey the "theoretical richness of Te Wharriki" (p.118), suggesting the new terminology might be applied to existing philosophies and practices.

In the years since its introduction, further provocation to New Zealand teachers' thinking, and to my own thinking about curriculum, has come from the growing strand of commentary and critique of Te wharriki, particularly since the turn of the century. While Cullen (1996) and McNaughton (1996) gave early notes of caution about the mix of theoretical perspectives, the predominant reaction was enthusiastic acclaim, and other critique appeared only slowly. An edited collection of critical commentary on the curriculum was not published until 2003 (Nuttall, 2003). Cullen's early comments on the intertwining of theoretical perspectives were elaborated and extended in the following years by Hedges (2003), Fleer (2003b) and Hill (2005). May and Carr had themselves identified four 'guides' in the planning_Eriksen, Piaget, Vygotsky, and Bruner (Carr \& May, 1996)—but as Cullen (2003a) noted, academic and professional debate focused on "its sociocultural and postmodern ethos" (p. 271).

Other aspects of Te whäriki that drew critique were the implied role of the teacher, and the question of children's content knowledge. In 1996, Stuart McNaughton noted that, while the new curriculum significantly redefined what constituted quality learning/teaching interactions, the implications for teachers were less clear. More recently Cullen (2003a) described the document as being "difficult to interpret as a guide to practice" (p. 271). It has also been argued that there is a need for increased subject knowledge for teachers (Hedges \& Cullen, 2005); Broström (2003) saw the absence of an "explicit discussion and formulation of aims, goals, and educational content" (p. 219) as a shortcoming and suggested Te whäriki lacked the "future-oriented content" (pp. 219-20) that is a necessary part of the process of involving citizens in democracy in order to bring about social change.

A residual caution about how effectively Te whäriki is implemented in centres continues. In 2003 Cullen wrote:

...it could still be argued that the ideals of Te Whäriki have barely touched many of our early childhood centres, and that many programmes today look remarkably like those of the 1980s and early 1990s, when the terms "free play" and "developmental" dominated the discourse of early childhood education. (2003a, p. 272) 
As discussed earlier, in-depth research studies in the $21^{\text {st }}$ century (Brennan, 2005; Hedges, 2007; Jordan, 2003; Nuttall, 2004) and commentary (Hill, 2001, 2005) confirm this anxiety and suggest that, more than a decade after the introduction of the final version, some teachers are still grappling with the implications of the sociocultural approach and the concept of curriculum contained in Te whäriki. The sense of unease about the extent to which teachers have shifted to a sociocultural framework is a theme that will re-emerge in this thesis.

Such critique of Te whäriki needs to be seen in context. First it needs to be seen within the context of continuing acclaim for the document both nationally and internationally. Writing in 2003, Cullen called it "trail-blazing" and "visionary" (2003a, p. 284) and acknowledged that wide-spread adoption of sociocultural concepts, and the thriving research culture had allayed some earlier reservations. Internationally, the tributes (e.g., Anning \& Edwards, 2006; Nutbrown, 2006a) continue.

The critique is also evidence of a developing maturity within the sector. The new curriculum contributed significantly to this by acting as "the catalyst for an emerging analysis of dominant beliefs and practices" (Cullen, 2003a, p. 269). Other government-initiated moves have enhanced this process. A governmentfunded review of research into early childhood teaching (Farquhar, 2003) provided a summary of research evidence. More significantly, a Strategic Plan (Ministry of Education, 2002) outlining a ten-year development plan for the sector (2002-2012) introduced the Centres of Innovation scheme through which early childhood settings were selected on a three-yearly cycle and funded to enable teachers and researchers to collaborate on projects that "showcase excellence and innovation in ECE" (Ministry of Education, 2002, p.8). Their reports (Meade, $2005,2006,2007)$ have contributed to an increased awareness of the complexity and challenge of early childhood education, which has been a key driver behind the government policy for fully teacher-led services. The presentations and reports from Centre of Innovation teams have also added to a growing enthusiasm for undertaking research, and an unprecedented openness to critique and debate within the sector (e.g., Hill, 2001, 2003, 2005; Keesing-Styles, 2002; Surtees, 2003). This thesis emerged from and reflects that climate. 


\subsubsection{Including children's voices in assessment and evaluation}

A reconsideration of the way children are positioned was the other area of focus that influenced the thesis topic. Reflecting international trends (described later in this chapter) there has been growing recognition within New Zealand of the need to include the children's perspectives both in assessment (e.g., Carr, 2001) and in research (e.g., Hedges, 2002a; Smith, Taylor \& Gollop, 2000).

In 1993, a New Zealand survey found less than half the centres surveyed had completed written assessments of children, and only a handful included children's perspectives (Wilks, 1993). Fifteen years later, this situation has changed dramatically. The introduction of the credit-based learning story assessment framework, which focuses on children's learning dispositions (Carr, 1998; Carr, 2001) and links them to the Te whäriki framework of goals was a fundamental shift (Carr, Hatherly, Lee \& Ramsey, 2003). This new framework promotes narrative observations (called "learning stories") in place of quantitative observation techniques and the 'impartial' running record. Learning stories provide an avenue for presenting children as learners within a sociocultural framework, with a focus on the child as competent and confident and developing the dispositions to learn. This change in assessment approach was also associated with a trend for keeping learning portfolios for each child (records, usually in photographs, words, and artwork that record significant learning moments in their centre lives). Increasingly teachers are encouraged to use children's voices and to give children an active role in contributing to their portfolios (Hill, 2001; Lawrence, 2004). In 2005 the release of Kei tua o te pae/Assessment for learning: Early childhood exemplars (Ministry of Education, 2004b) provided teachers with a rich resource of examples of learning stories. The fourth booklet in the resource focuses on the child's perspective, arguing for it in terms of its contribution to the efficacy of learning and as recognition of children as social actors in their own right.

A similar change has also taken place in evaluation, with the introduction of the succinct "child's questions", based again on the Te whāriki strands (Carr, May \& Podmore, 1999; Podmore \& May, 2003), which guide teachers to think from the perspective of the child when evaluating their own practices. The commitment to foreground children's voices through this thesis is an affirmation of the enormous shift teachers have made in their assessment and evaluation processes since the early 1990s. 


\subsection{International strands of thinking and research within early childhood education}

Internationally, a similar range of stimulations and challenges have impacted on thinking around these two intersecting areas - the defining/redefining of what constitutes curriculum for young children, and the reconceptualising of children as social actors in their own right. The writings of those in the reconceptualist movement, and the philosophical and pedagogical approach of the early childhood education centres of Reggio Emilia, Italy (Edwards, Gandini \& Forman, 1994) have both had significant impact. Both reflect a commitment to sociocultural approaches, contain elements that disturb assumptions about children and teaching, and challenge the status quo. A third area of literature, which contributed to the thesis methodology, was research which foregrounded children's perspectives.

\subsubsection{Reconceptualising early childhood education}

As part of the broad reconceptualisation movement referred to in the opening paragraphs, through the 1990s a growing cadre of early childhood scholars called for a reconceptualisation of early childhood education, challenging others to problematise their thinking about children and about how adults have traditionally taught them, and to bring "a crisis of thinking” (Dahlberg, Moss \& Pence, 1999, p.17) to the field. The underlying motivation was to create social change in order to improve the lives of children (Jipson, 2001). The majority of reconceptualist writers have been American, although Australian work has increasingly appeared (e.g., Campbell \& Smith, 2001; Hughes \& MacNaughton, 2001). New Zealand work with a reconceptualist focus has been rarer, and includes work by Ritchie (2001, 2005), Gibbons (2007) and Farquhar (Farquhar \& Fleer, 2007). The spread of ideas reflects in part the volume of writing emerging under this banner. Publishing houses Peter Lang and Routledge have both produced a series of books, and a journal (Contemporary Issues in Early Childhood) was launched in 2000. While this continues to be a largely scholarly discussion, the overlap between the themes in the reconceptualist literature and those arising in writing about Reggio Emilia (discussed in the following section) has assisted in spreading the reconceptualists' ideas.

Three themes in the reconceptualist literature are particularly relevant for this thesis. The first two, the recognition of how children have been positioned as 
'other than' adult and their voices silenced, and the understanding that "the sometimes insidious culture of early childhood education" (Jipson, 2001, p.9) can function to silence children, have become increasingly important during the process of analysis and writing. The third, discussion of how research in early childhood education might be reconceptualised, provided challenges to consider in planning the methodology. (For the sake of coherence, the three themes are discussed together here.) However, what initially was most influential for me was their willingness to challenge traditions and to step outside familiar frameworks. Their collective example bolstered my confidence in embarking on a topic that might challenge assumptions of pedagogy and practice.

The rejection of child development theories (e.g., Bloch, 1992; Cannella \& Viruru, 2004) and recognition of the blinkering effects of this theoretical framework-it is "embedded in a system of reason that shapes and fashions how educators "see," think, talk, and act toward teaching, children and schooling" (Bloch \& Popkewitz, 2000, p.7)—are a central tenet. That approach is seen to have led teachers to make decisions for children unquestioningly, "constructing the languages with which they should speak, the materials that they should use, and the ways of thinking that should control their bodies" (Cannella, 1997, p.165). Within early childhood education, the debate has focused on a rejection of developmental psychology, and subsequently of child-centred curriculum as being "an adult-dominated arena, heavily developmentally laden with inherent biases and modernist views" (Yelland \& Kilderry, 2005, p.4).

The constructions of 'child' and 'childhood' that are embedded within early childhood education practices have been challenged (Cannella, 1997; Cannella \& Viruru, 2004; Dahlberg \& Moss, 2005):

We have constructed the field of early childhood education based on the notion of "child" as psychologically and physically distinct from other human beings. Living within this construct, those who are younger have been controlled, oppressed, labeled, and limited. Their voices have been silenced under the weight of "adult" psychological, education, and policy constructions of and for them. (Cannella, 1997, p.162)

Lenz Taguchi (2006) has advocated adopting an 'ethic of resistance' which requires scrutinising "assumptions and taken-for-granted notions we bring with us (often without awareness) as we engage in our daily work with children" (p.259). Like others, Dahlberg et al. (1999) draw on the writings of Malaguzzi, first director of the Reggio Emilia centres, and suggest his alternative image of a "rich 
child, active, competent and eager to engage with the world" (cited in Dahlberg et al., 1999, p.7, italics in original) offers a way forward. While recognition of the positioning of children as dependent and incompetent is a continuing theme in reconceptualist writing, increasingly the focus has shifted from children per se, to children from diverse groups who are doubly disadvantaged by the pervasive discourses of early childhood education. It was, however, the positioning of children as other than adult that engaged me. It seemed likely that exploring what constituted the boundaries of curriculum in a centre might reveal unrecognised ways in which children were disempowered.

Research methodologies based on developmental perspectives have also been challenged by reconceptualist scholars (e.g., Bloch, 1992; Cannella, 1997; Dahlberg \& Moss, 2005; Soto \& Swadener, 2005). Among the criticisms leveled are their dependence on Western ways of thinking, their failure to accommodate human diversity, to recognise the social and cultural values implicit in research decisions, and "to consider whose interests are served by the knowledge they produce" (Jipson, 2000, p.169). Such research has been described as a form of modernist colonisation that must be deconstructed, perhaps rejected, and at least reconceptualised (Viruru \& Cannella, 2001). There is, instead, a commitment to newly evolving critical orientations within research, and to multiple ways of knowing (Soto \& Swadener, 2002). The difficulties of both envisaging and engaging in such research are acknowledged: "This conceptualization of research is not easily discussed and certainly does not fit with beliefs in models or preplanned controlled design, because it involves suspending traditional understandings" (Cannella \& Viruru, 2004, p.147). A commitment to finding methods that enable children's voices to be heard is a recurring but not constant theme within these discussions.

Reading the work of reconceptualist scholars was valuable as I framed the thesis topic because it indicated an in-depth investigation of the reality of children's experience of aspects of curriculum might add a further dimension to what was largely a theoretical discussion. My commitment to empowering children within the research process was affirmed, but my thinking about other aspects of research design was challenged, and continues to be challenged, by these writers. In particular I am aware of an unresolved tension in relation to the adults in the centre. This tension will be explored in Chapter 4. 
For teachers, the centres in Reggio Emilia, Italy (Edwards et al., 1994) have been a more direct source of inspiration through the 1990s, particularly in the repositioning of children as strong and competent and the implications this has for their practices. There was considerable interest in the United States and Sweden, but few New Zealand teachers chose to describe their settings as 'Reggio' centres; a more common response was that a pedagogy could not be transferred from one cultural context to another and that structural differences would make their practices difficult to implement (based on Bayes, 1999, pp.11-12). However, the emphasis on documentation and display of children's learning, and on the project approach, "in which children and teachers together examine topics of interest to young children in great depth and detail" (Katz, 1994, p. 36), have been influential. Like others, I drew inspiration from these Italian centres as the ideas of the founder, Loris Malaguzzi (Edwards et al., 1994; Malaguzzi, 1993; Rinaldi, 2006) became increasingly familiar and were incorporated into teacher education programmes. Perhaps their greatest significance was that, like the writings of the reconceptualists, they offered thought-provoking challenges to accepted practices which could be used to problematise our own settings. The vision they offered of an alternative model of early childhood education influenced the critical approach taken in this thesis.

\subsubsection{Including children's voices in research}

The concerns expressed by reconceptualist scholars about the role of children in research is reflected in a much wider range of international literature in which assumptions about the power differential between adults and children, the prerogative of adults to make 'right' decisions on behalf of children, and the superiority of adult knowledge are increasingly questioned. There has been a surge of literature that advises on procedures for including children in research (e.g., Clark, 2004; Christensen \& James, 2000; Einarsdottir, 2007; Lewis, Kellett, Robinson, Fraser, \& Ding, 2004; Lewis \& Lindsay, 2000). Particularly influential for me were the Australian early childhood researchers writing about the ethical and methodological challenges of research with young children (Danby \& Farrell, 2004; Fasoli, 2003; Krieg, 2003; MacNaughton, 2003a; Sorin, 2003; Sumison, 2003). Wider reading introduced me to others who were including the voices of children and/or taking the perspective of children: from Europe (e.g., Einarsdottir, 2005, 2007; Formosinho \& Araujo, 2006), from the United Kingdom (e.g., James, 2005), from the United States (e.g., Wiltz \& Klein, 2001), and from Asia (e.g., 
Hadley \& Nenga, 2004). Reading their work helped clarify my thinking about my research role with children, and offered additional ideas for methods. Finding how rare it was for researchers to include children of 2 years or younger suggested this was an area of methodology where this thesis might make a contribution.

This section has described the multiple ways in which established beliefs about childhood, early childhood education, curriculum and research have been challenged in the national and international academic literature since the start of the 1990s and the rich array of ideas and possibilities that have been introduced. Yet my experience as a teacher educator, both in teaching and in visiting students on practicum, suggested that a "current dominating language" (Dahlberg et al., 1999, p.3) still existed within much of New Zealand early childhood education, that there was continuing loyalty to familiar patterns of interaction and to familiar activities and resources (Cullen, 2003a; Hill, 2001).

It is important not to underestimate the scale of challenge confronting the profession. While reconceptualisations of the child, and of the consequent role of the adult, have become increasingly familiar in early childhood academic writing (e.g., Cannella, 1997; Dahlberg et al., 1999; Fleet, 2002; Woodrow, 1999) the task of re-examining deeply embedded assumptions, of disentangling long-held attitudes, of reworking what Smith and Taylor (2000) called the "balance between agency and dependency" (p. 4) in interacting with children, is enormous. Fleet (2002) talks of "jarring the expected formula for working in early childhood settings" (p.19) as a way of making hidden assumptions visible, and rendering the familiar 'strange'. On a personal level I knew the struggle of shifting theoretical frameworks intellectually, but had not personally experienced the additional challenge teachers face in reworking accustomed daily practices.

\subsection{The thesis aims, the research questions and the research setting}

These then were the personal, professional and policy milieu which informed the shaping of this thesis. There were, as this chapter has shown, liberating movements within early childhood academic research and writing, nationally and internationally, that supported the proposed focus and design of the study. Recent New Zealand research had indicated the value of qualitative in-depth studies in centre settings and had suggested many teachers were still grappling with the challenges of implementing Te whäriki (e.g., Brennan, 2005; Hedges, 2007; 
Jordan, 2003; Nuttall, 2004), but no research had focused on the boundaries of curriculum. It was hoped such work would contribute to the growing understanding of the reality of curriculum implementation in New Zealand centres. Focusing on children's perspectives as well as including younger children was hoped to build on and extend the methodological foundations laid by others (e.g., Carr, 1997a, 1997b; Hedges, 2002b; Greenfield, 2004). It was also hoped that it would offer further insight into children's experiences of centre life. More broadly, it also seemed important to undertake research that would contribute to the questioning of the everyday and the ordinary within early childhood education, in order to generate discussion about how it might be transformed. It was hoped this thesis might be part of a movement that assisted teachers and teacher educators to re-examine assumptions, and to envision new ways of educating young children in the $21^{\text {st }}$ century.

\subsubsection{The aims for the thesis}

The thoughts outlined above translated into a list of aims for the thesis.

\section{The aims for the thesis}

The first relate to the single research setting:

- Provide insight into where the boundaries of curriculum lie

- Describe the process of demarcation, and show how children experience and influence this process

- Within the context of the above, offer insight into how indirect/unintended aspects of curriculum are experienced, influenced and enacted by children

- Provide insight into children's thoughts and feelings about their day-today experiences of centre life.

Broader purposes were to:

- Contribute to accumulating research knowledge of curriculum implementation in New Zealand centres in the early $21^{\text {st }}$ century

- Build on and extend the foundations laid by others in exploring strategies for including young children as active participants in research

- Be a strategy of dislocation for others in assisting them to re-examine assumptions about early childhood education. 


\subsubsection{The research question}

The thesis began with a broad overarching research question:

What are the scope and boundaries of the curriculum that young children experience and enact within the early childhood education setting?

The question was later narrowed and rephrased as:

What are the boundaries of the curriculum in the early childhood setting, and how do young children experience, influence and enact these boundaries?

The original research focus had been guided by subsidiary layers of questions, directing attention to the indirect aspects of curriculum. When the scope of the overarching question was narrowed, these were also amended and became:

How do the "indirect", less noticed or unacknowledged aspects of teaching and learning that are occurring influence the boundaries of curriculum that children experience?

The following two questions contributed to answering this question:

How do the hidden aspects of the curriculum, which are implicit in the ways the setting is organised, the kinds of resources provided, and the behaviours of the adults, affect the boundaries of the curriculum that children experience?

How does the informal and less noticed or unacknowledged learning and teaching that occurs among children affect the boundaries of the curriculum that they experience?

The final subsidiary question remained unaltered:

What potential aspects of learning and teaching, i.e., the null curriculum, are being excluded from the curriculum that young children experience and enact?

A variety of approaches were used to generate data. Over the first days photographs were taken to provide a detailed record of the environment as a route to identifying aspects of the indirect/hidden curriculum implicit in the design, organisation and resourcing. As participants became comfortable with my presence, I began to use fieldnotes (and sometimes photographs and audio 
recordings) to record children's and teachers' interactions with a continuing focus on identifying both the wider, and less noticed, content of the learning/teaching that was occurring for children, and also the process by which potential aspects of learning and teaching were excluded, i.e., the null curriculum (Eisner, 1985). This allowed the boundaries between what was defined as appropriate/inappropriate, relevant/irrelevant to be identified, and the influence that children might have in this process to be explored. Over time a series of strategies was introduced and developed that supported children in sharing their perspectives. During the period of data generation, the teachers' documentation of planned teaching and learning that was occurring through the period was photographed and partially transcribed. This data source indicated the contents of the inner circle described in the opening paragraphs, the intended learning and teaching, but was less central to the research question and so to the analysis. Towards the end of the period six focus children were identified, and an interview was conducted with a parent of each. The emerging codes and categories in the ongoing analysis guided the evolving directions of the research. (Chapter 4 contains a full description of the methodology.)

\subsubsection{The research setting}

'The centre' is the pseudonym suggested by teachers at the childcare centre that generously, and bravely I think, agreed to participate in this undertaking. The centre building, single storey and purpose built, is sited in a grassed area behind a workplace complex in a suburb of tree-lined streets. Open five days a week, it is licensed for 18 over-two-year-olds and 8 under-two-year-olds. Waiting lists are long for under-two positions but older children can sometimes be placed immediately. At the beginning of the study there were 37 children on the roll, both part-time and full-time, ten of whom were under two. Many families lived in the middle class suburbs in the vicinity or to the south of the centre. Nine women worked in the centre, seven teachers, a manager and a cook. The children and the adults will be introduced in more detail in Chapter 4 .

While it was decided to complete this research study in a single setting, in taking this decision it was anticipated that this site would provide illustrations of assumptions and practices that are accepted in many other early childhood centre settings (Denscombe, 2007), and particularly those that identify as being 'mainstream'. The thesis is less likely to reflect the programmes of centres with a 
strong philosophical orientation that distinctively shapes their practices and programmes within the broader framework of Te whäriki. Examples of such centres are: ngā kohanga reo which provide immersion Māori language for Māori children; Pacific Island early childhood education centres which provide immersion in the languages of the Pacific Nations peoples living in New Zealand; and Steiner and Montessori centres which have philosophical orientations that distinguish them from mainstream early childhood education centres.

\subsection{Overview of the thesis}

This chapter has introduced the thesis topic and described the personal and professional journeys that influenced it. In particular, it has shown how events and thinking, nationally and internationally, have combined to shift established ways of thinking about curriculum, about children, and about the practices of early childhood education. The ways in which the gathering threads of these paradigm shifts contributed to the formation of this thesis topic have been described. The research questions have been defined and the way in which they evolved explained. The centre that participated in the research has been introduced.

Critical pedagogy and the sociology of childhood are introduced in Chapter 2, their contribution to the thesis is described, and relevant literature is reviewed. Within the discussion of the impact of critical pedagogy, use of the concepts of the hidden and the null curriculum in relation to early childhood education is considered, and the definitions adopted for these linked concepts in this thesis are given. The discussion of sociology of childhood includes a review of how others have included the perspectives of young children within research. Finally, activity theory is introduced, with a particular focus on the concepts which have been used as sensitising concepts in this thesis-the physical resources and environment, the roles, and the routines, rituals and rules. The role of the 'strategies of dislocation' which were devised for this thesis is introduced.

In Chapter 3 the literature and research relevant to two aspects of this thesis are reviewed. The first is a review of literature relating to the boundaries of curriculum, and here the focus is particularly on what might constitute null curriculum. The second area of literature refers to young children's peer groups and peer culture, and here the focus is particularly on ways in which children may influence the scope of curriculum. 
Chapter 4 opens with a discussion of the generic inductive qualitative model (Hood, 2007) and the decision to use that approach in guiding aspects of the inquiry, data generation and analysis. The following sections describe four sequential processes. The first covers the refining of the topic into a set of targeted questions, and the design of the methodology. The second section focuses on the practical and ethical procedures involved in identifying a setting, negotiating access to the centre and gaining ethical consent/assent from participants, and the day-to-day reality of living those ethical commitments. The third section describes the gathering of data, and the development and refinement of the research strategies used with children. The final section discusses the process of data analysis, both during and after the period of data gathering.

The next four chapters contain the findings, the first three being structured around a concept drawn from activity theory. Chapter 5 opens with a description of the current scope of curriculum in the centre, and then introduces the findings in relation to the environment and the physical resources. The first emergent theme is introduced here; this is that an assumed demarcation between adults and children was found to underpin many facets of centre life, and that this was a central source of curriculum boundaries.

In Chapter 6 analysis of the data from the perspective of teachers' and children's roles is presented. Teachers' roles, from the perspective of children, are described, and then children's roles as friends, as boys/girls, and as peer learners/teachers. The second emergent theme of the thesis is introduced here: it was found that many interactions and relationships within the centre were characterised by a dialectic quality with expressions of companionship and community in tension with expressions of control/power. It is argued that relationships were at the heart of curriculum for children, and that both these elements were central curriculum concerns for them.

In Chapter 7 the rules, routines and rituals of the community are used as the lens through which to examine the data. Here there is a focus on children's and teachers' use of rules, on three of the centre routines, and on the ritual of celebrating a fifth birthday. Collectively these show how the norms or 'rules' impacted on children's experience and reinforced the boundaries of curriculum. 
Although the two key themes of the thesis were thrown into relief through this analysis, there were also elements that offered ideas for potential future change.

In Chapter 8, the null curriculum is introduced. Here the potential aspects of curriculum that arose or might have arisen but which either teachers or children chose not to include are described. A significant finding was that children and teachers had differing interpretations of what constituted null curriculum, which provided reinforcement for the embedded demarcation between them.

The final chapter recapitulates the key findings, and reviews the list of aims set for this thesis (in section 1.6.1) in order to assess its success. The research strategies developed for talking with children are reviewed. Findings from the thesis are aligned with findings from other recent New Zealand qualitative studies in similar settings as one way of addressing concerns about the reliability of such small-scale research projects (Cullen, 2003b). With the drawing together of the final arguments, implications for the profession, for both practice and research, are outlined.

This chapter has introduced the thesis topic, and the paradigm shifts within early childhood education nationally and internationally that have helped to shape it. The next chapter will explore two wider theoretical frames which overlap and which stretch beyond the area of early childhood education, both of which have in different ways significantly influenced and informed aspects of this research. 


\section{CHAPTER 2}

\section{THE THEORETICAL FRAMEWORK}

Schools should never impose absolute certainties on students. They should stimulate the certainty of never being too certain. (Freire, in Freire \& Macedo, 1987, p.57)

What were the theoretical and philosophical approaches that were used as the context for this thesis? The answer to that question, which is the substance of this chapter, will reveal some of the ways in which a desire to "stimulate the certainty of never being too certain" (Freire, 1987, p.57) has influenced all stages of the design and process.

Critical pedagogy was central in the original impetus for the thesis. It was within this discipline that the concepts of the hidden and null curriculum had been most extensively used, and this was the context within which the research topic and questions were framed. And critical pedagogy remained crucial throughout in providing a framework in which to consider the issues of power that were found to be a recurring feature of the data at both the macro-level of the institution and at the micro-level of interpersonal relationships. Critical pedagogy is introduced in the first section of the chapter.

Approaches underpinned by the sociology of childhood came later with the decision to foreground the perspectives of children. Initially such understandings were valuable in practical terms in the design of the methodology and as a source of research strategies to use in working with children, but it became increasingly significant theoretically as generational issues emerged as a dominant aspect of the findings. The sociology of childhood, and the role it played within this thesis, is described in the second section of this chapter. While at times weaving these dual frameworks together was challenging, they complemented each other, and although it would have been possible to select either as the single framework, siting the thesis at a point of intersection between the two seemed a stronger if less straightforward option.

I briefly considered including activity theory as a third dimension to the theoretical framework, but eventually decided to use it as the source of three sensitising concepts (Bowen, 2006). The third section of this chapter includes a 
definition of sensitising concepts, and describes the three constructs that were used in this way. The unit of analysis for the thesis is introduced here.

Finally, the concept of strategies of dislocation is explained, and the three strategies that were used in this thesis are described. The first, the concepts of the hidden and null curriculum, came from critical pedagogy. The second was the foregrounding of children's perspectives. The use of sensitising concepts drawn from activity theory was the third of these strategies.

While not considering myself an expert in sociocultural theory, this approach had guided my professional thinking for the past 15 years, and I had always assumed this would provide the theoretical context for the thesis. However, I was also aware that adopting this as the theoretical frame of reference was akin to putting a fish into water; the familiarity might hamper my ability to see what was "hidden in plain sight" (Margolis, Soldatenko, Acker \& Gair, 2001, p.2). Having previously experienced the paradigm shift from Piagetian to Vygotskian thinking I was aware how such frameworks can become filters that structure thinking. Choosing a less familiar framework fitted with the intention of the thesis.

\subsection{Introduction to critical pedagogy}

As outlined above, this section serves several purposes. It introduces critical pedagogy as providing the theoretical context for the thesis in which the research questions were framed. Because issues of power were found to be central in the data, this section includes an outline of how the construct of power has been addressed within this literature. It also describes the evolution of the concepts of hidden and null curricula, and explains the definitions that were developed here.

\subsubsection{Critical pedagogy}

Critical pedagogy arose from within the broader field of critical theory, which in turn had its origins in the theoretical tradition developed by a group of scholars (Horkheimer, Adorno and Marcuse) known as the Frankfurt School who were connected to the University of Frankfurt before World War II (Kincheloe, 2004). Critical theory is not easily defined given the many and evolving versions; moreover, providing a specific definition is contrary to the central thrust of avoiding defining blueprints (Kincheloe \& McLaren, 2005). While the term is used to cover a range of groups, Kincheloe and McLaren (2005) offer a definition 
of a criticalist as one who uses their "work as a form of social or cultural criticism" (p.304); who accepts that "all thought is fundamentally mediated by power relations" (p.304); "that facts can never be isolated from the domain of values or removed from some form of ideological inscription" (p.304); "that language is central to the formation of subjectivity" (p.304); and that in any society some groups are privileged over others.

Paulo Freire, in his educational work with Brazilian peasants, is acknowledged with providing the genesis for critical pedagogy (Kincheloe, 2004). Freire recognised the inherently political nature of education, and his focus on liberation positioned learning as "inseparable from individual empowerment and social change" (Kincheloe, 2004, p.71). But Freire also recognised that the pedagogy "must be forged with, not for, the oppressed" (Freire, orig 1972/1990, p.25, italics in original) and cautioned "it is necessary to trust in the oppressed and in their ability to reason. Whoever lacks this trust will fail to bring about (or will abandon) dialogue, reflection and communication, and will fall into using slogans, communiques, monologues and instructions" (Freire, 1972/1990, p.41). Freire rejected any attempt to reduce his methods to a set of strategies, insisting that practices needed to be adapted for each individual context (Keesing-Styles, 2003).

However, it was with Giroux's writings in the last decades of the $20^{\text {th }}$ century that what is now known as critical pedagogy emerged from critical theory, with a focus on the relationship between power and knowledge, and a goal of disclosing and challenging the role that education plays in social and political life. Like critical theory, critical pedagogy is considered to be a broad and evolving framework which encompasses a myriad of approaches (Kincheloe, 2004). However, a number of themes are consistently identified:

- Every aspect of education is recognised as being politically contested.

- Knowledge is seen as never neutral, but rather as a social construction (McLaren, 2007) and the critique of positivism is seen as central (Kincheloe, 2004).

- The concept of hegemony, developed by the Italian political activist Gramsci (Apple, 2004; Kincheloe, 2004; McLaren, 2007), is central. Hegemony is "a struggle in which the powerful win the consent of those who are oppressed, with the oppressed unknowingly participating in their own oppression" (McLaren, 
2007, p. 203). Dominance is gained and maintained not through physical force or coercion, but rather through consensual social practices and the oppressed come to accept their social status as "natural, necessary, or inevitable" (Kincheloe \& McLaren, 2005, p.304). Counter hegemony is the resistance to, and struggle against, such hegemonic controls.

- Critical pedagogy is dedicated to transformation, to social and educational justice and equality (Kincheloe, 2004; Giroux, 2001). Education is seen to have potential both as a force for domination and emancipation (Giroux, 1997; McLaren, 2007). For critical pedagogues it is not sufficient to reflect critically on the world, one must also be willing to act to change it.

Critical pedagogy has not been without its critics. Critical pedagogues have been criticised for using apparently 'universal' categories and explanations (Gore, 1993), for failing to address, or include the voices and concerns of women and other groups (Burbules \& Berk, 1999; Darder, Baltodano, \& Torres, 2003; Gore, 1993), for writing theoretically and inaccessibly (Darder et al., 2003), for being "highly abstract and utopian" (Ellsworth, 1989, p. 297), for concentrating on macro-levels of power while ignoring the micro power relations within the act of instruction (Gore, 1993), and for failing to address practical issues (Gore, 1993). More fundamentally, those working in this field have been charged with perpetuating the relations of domination they have challenged through their inadequate reflection on the implicit power dynamics inherent in the concept of empowerment (Ellsworth, 1989; Gore, 1992), and with failing to recognise the regime of truth within which critical pedagogy operates (Gore, 1992). Because the findings in this thesis concern children, address both macro and micro power dynamics within the centre community, and within the apparently empowering relationships between teachers and children, some of these charges raised concerns that were pertinent to how critical pedagogy was used in the thesis.

This section has provided an introduction to critical pedagogy; for more detailed introductions see Kincheloe (2004) or Darder et al. (2003).) However, before introducing the hidden curriculum and the null curriculum, the next section describes how the construct of power has been addressed within the literature of critical pedagogy. 


\subsubsection{Power}

Issues of power are central within critical pedagogy, frequently described within a framework of domination and oppression, and often as portraying a process of hegemony (Kincheloe, 2004; McLaren, 2007). For critical pedagogues all educational institutions are seen as "sites centrally involved in the production and formation of subjectivity, agency, systems of value, regimes of truth", and as such are "deeply invested spaces for hegemonic and counterhegemonic contestation" (Janmohamed, 1994, p.247). These dimensions of power are seen often to be as invisible to the dominant group as they are to the subordinate, concealed behind apparently benign educational discourses. A pedagogy can be "totalitarian and oppressive" even when it operates "in the name of democracy and justice" (Kincheloe, 2004, p.2). Such discourses can become "a cultural encasement of meanings, a prison-house of language and ideas, that is "freely" entered into by both dominators and dominated" (McLaren, 2007, p.203).

However, critical pedagogues have also been criticised (Gore, 1992) for dichotomising empowerment and oppression, and for their use of the "dualisms of power/powerlessness, and dominant/subordinate" (Gore, 1992, p.61). In making this criticism Gore (1992) draws on the ideas of the French philosopher, Foucault, and since her criticism his ideas have increasingly been incorporated by those working within the field (e.g., Giroux, 1997; McLaren, 2007).

The writings of McLaren (2007) offer an example of how Foucauldian concepts are incorporated within critical pedagogy in order to strengthen and complicate the theoretical understanding of power, and its relationship to knowledge, and provide a portal for those ideas within this thesis. McLaren incorporates four concepts from Foucault's writings into his discussion of power-the notions of power, discourse, regimes of truth, and truth. Foucault's work is introduced with the comment that it is crucial to "understanding the socially constructed nature of truth and its inscription in knowledge/power relations" (McLaren, 2007, p.209). McLaren describes Foucault's conception of power as coming "from everywhere, from above and from below" and being "inextricably implicated in the microrelations of domination and resistance" (p.209). While not explicit, the underlying Foucauldian concept of power is not as something possessed, but rather as something which is exercised, and interwoven into relationships (Barker, 1991; Gore, 1992). The second concept introduced is the notion that "power relations 
are inscribed in what Foucault refers to as discourse or a family of concepts" (McLaren, 2007, p.209, italics in original). McLaren defines discursive practices as "the rules by which discourses are formed, rules that govern what can be said and what must remain unsaid, and who can speak with authority and who must listen" (p.209, italics in original) and clarifies that it is not simply the language, but also the practices, the patterns of behaviour that are regulated. McLaren's synopsis glosses over Foucault's recognition of the potential dangers inherent in all discourses, including those which aim to liberate. McLaren introduces the third Foucauldian notion, 'regimes of truth', describing them as the discourses produced by the dominant culture: "In a classroom setting, dominant educational discourses determine what books we may use, what classroom approaches we should employ... and what values and beliefs we should transmit to our students" (pp.209-210). The final concept from Foucault which McLaren draws on is his understanding of truth, which builds on an earlier discussion of knowledge as "socially constructed, culturally mediated, and historically situated" (McLaren, 2007, p.210). He quotes from Foucault:

Truth is a thing of this world: it is produced only by virtue of multiple forms of constraint. And it induces regular effects of power. Each society has its regime of truth, its "general politics" of truth: that is, the types of discourse which it accepts and makes function as true; the mechanisms and instances which enable one to distinguish true and false statements, the means by which each is sanctioned; the techniques and procedures accorded value in the acquisition of truth; the status of those who are charged with saying what counts as true. (Foucault, cited in McLaren, 2007, p.210)

McLaren draws out the implications for education. "Teachers need to recognize that power relations correspond to forms of school knowledge that distort understanding and produce what is commonly accepted as "truth", (McLaren, 2007, p.211, italics in original).

As well as providing an example of how understandings of the construct of power within critical pedagogy have been extended through incorporating concepts from Foucault, this summary also demonstrates the growing acknowledgement of the "multiplicity and contradiction" (Gore, 1992, p.61) of the real world, which has been seen as lacking in the field. Others have made similar charges; in 2004 Kincheloe wrote "This theme of complexity is central to any critical pedagogy that works to avoid reductionism. Critical pedagogues who take complexity seriously challenge reductionistic, bipolar, true-or-false epistemologies" (p.37) and "critical teachers come to recognize the complexity of the lived world with its 
maze of uncontrollable variables, irrationality, non-linearity, and unpredictable interaction of wholes and parts" (p.37). Kincheloe's words reaffirmed the complexity of the task undertaken in this thesis, served as a warning of the perils of simplistic conclusions and were to haunt me through the process of analysis, as I sought to sift patterns from the data.

The final task to be undertaken in this section is to introduce the paired constructs, the hidden and null curricula, describe their evolution and current use, and explain how they are defined within this thesis.

\subsubsection{The hidden curriculum}

While references to the hidden curriculum usually appear in the literature of critical pedagogy (e.g., Apple, 2004; Giroux, 2001; McLaren, 2007), it arose in a different arena. Philip Jackson (1968) is acknowledged with having developed the concept to draw attention to those aspects of schooling which were not part of the formal subject matter, but elements of socialisation. He labeled "crowds", "praise", and "power" (p. 33) as the three key elements of hidden curriculum. The crowded conditions of classrooms meant students were learning to cope with experiences of "delay, denial, interruption and social distraction" (Jackson, 1968, p.17). School life meant a student had to be accustomed to being evaluated, and sensitive to the assessments others were making. Finally, he saw the power differential between teacher and students as the "most salient feature of the social structure of the classroom" (p.29). Jackson understood that learning to adapt and respond to these three features in ways deemed appropriate was essential to a student's satisfactory progress.

Discussion of the concept of the hidden curriculum has flowed and ebbed since Jackson's work, and definitions have varied, reflecting divergence in the underpinning ideological assumptions. While a full discussion of the literature relating to the use of the concept is beyond the scope of this thesis, Skelton (1997) and Margolis et al. (2001) provide useful overviews. Giroux (2001) describes three broad approaches that have emerged in relation to the concept—-traditional, liberal, and radical—and he and Skelton (1997) both postulate the need for a new approach. Skelton (1997) outlines the value of a postmodern approach, particularly drawing on the insights of Foucault. Giroux (2001) argues for an approach which acknowledges that hidden curricula are plural and that 
contradictions open spaces for student resistance. Such an approach is reflected in the writing of resistance theorists such as Apple, Giroux, hooks and McLaren, who all draw attention to the significance of agency and resistance (Margolis et al., 2001). However Giroux (2001) warns that analyses will "gain some theoretical mileage only when they move from description to critique" (2001, p. 47) and address the question of how the hidden curriculum functions as an agency of social control. He considers the concept will "have to be both redefined and resituated as a pedagogical concern" $(2001$, p.61) if it is to be an important theoretical concept within critical pedagogy, and that emphasis needs to shift to intervention and social action. To achieve this, he suggests, requires that the concept of the hidden curriculum:

- Occupy "a central ... place in the development of curriculum theory" so that the "notion of critique" (p.61) is embedded,

- Is used "to analyze not only the social relations of the classroom and school, but also the structural "silences" and the ideological messages that shape the form and content of school knowledge" (p.61),

- Is "linked to a notion of liberation, grounded in the values of personal dignity and social justice" (2001, p.61).

However, not all who write about the notion of the hidden curriculum remain optimistic for its future usefulness. Some suggest the tide of thought may be turning again, "idealism ... may have run its course" and that writers now "give full weight to the power of institutions to manage contestation, reproduce hierarchy, and resist change" (Margolis et al., 2001, p.17).

\subsubsection{Defining the hidden curriculum}

Deciding on the definition of the hidden curriculum to be used in this thesis was not straightforward. Gair and Mullins (2001) describe the concept as problematic, because it suggests a conscious duplicity, whereas it may more correctly be "not something that we must look behind or around in order to detect; in most cases it is plainly in sight, and functions effortlessly" (Gair \& Mullins, 2001, p.23).

"Hidden in plain sight" is the phrase Margolis et al. (2001, p.2) use to capture this apparent contradiction of undetected but obvious. 
Most of the definitions offered in the literature focus on the process by which messages are generated rather than on their effect upon recipients. For example, using the term 'implicit' rather than 'hidden', Eisner (1985) describes the implicit curriculum of a school as:

...what it teaches because of the kind of place it is. And the school is that kind of place through the ancillary consequences of various approaches to teaching, by the kind of reward system that it uses, by the organizational structure it employs to sustain its existence, by the physical characteristics of the school plant, and by the furniture it uses and the surroundings it creates. (p.97)

McLaren (1998) defines the hidden curriculum as "the unintended outcomes of the schooling process" (p.186) explicitly including not only the "rules of conduct, classroom organization, and the informal pedagogical procedures used by teachers with specific groups of students" (pp.186-7) but also the "teaching and learning styles that are emphasized in the classroom, the messages that get transmitted to the student by the total physical and instructional environment, governance structures, teacher expectations, and grading procedures" (p.187). Both McLaren (1998) and Eisner (1985) acknowledge positive aspects—Eisner suggests the implicit curriculum can teach "a host of intellectual and social virtues" (p.95).

Other definitions of the hidden curriculum give more prominence to students' mediation of the messages. For example, "the hidden curriculum is what students have an opportunity to learn through everyday goings-on under the auspices of schools, although teachers and other school people do not intend those learnings" (McCutcheon, 1997, p.188). Skelton's (1997) definition underlines the complexity of the students' reception of embedded messages: "The hidden curriculum is that set of implicit messages relating to knowledge, values, norms of behaviour and attitudes that learners experience in and through educational processes. These messages may be contradictory, non-linear and punctuational and each learner mediates the message in her/his own way" (p.188).

In this thesis, an unusually broad definition of the hidden curriculum is used for two reasons. One is that I wanted a definition that mirrored the distinction between "direct or indirect" in the Te whäriki definition of curriculum, cited in Chapter 1. The other is that I wanted to include what young children learn from each other as part of the hidden curriculum. Most definitions have been written in reference to schools, and the impact of the peer group is not included. However, while older children are likely to have out-of-school contact with peers and less opportunity for interaction with them 
during school, for young children being part of their peer group is often synonymous with being in the centre and the relatively unstructured programmes encourage such interaction. It therefore seemed likely that peer-group interactions would contribute significantly to the indirect curriculum. The definition used in this thesis draws on and extends the detail in Eisner's and McLaren's, the emphasis on individual mediation of meaning in McCutcheon's, and makes explicit the role of children's contributions. The definition is:

The hidden curriculum is that set of implicit messages relating to knowledge, values, norms of behaviour and attitudes that children experience while in the centre. These messages are located in and derived from the way the setting is organised physically, temporally and socially, the kind of resources provided, the informal and formal practices of the adults, and the ongoing interactions within that community of adults and children. Both adults and children contribute to the hidden curriculum, messages may be contradictory, and each person mediates the message in her or his own way.

Defining the hidden curriculum was a first step in the process of discerning the boundaries of curriculum, both covert and overt, within the centre, and of identifying how children experienced, influenced and enacted those boundaries. In the final part of this section, the concept of the null curriculum is introduced and defined. A review of how this construct has been used by scholars of early childhood education is included in Chapter 3.

\subsubsection{The null curriculum}

Defining what constitutes the null curriculum in an early childhood education setting seems on the surface a less ephemeral task than trying to disentangle the elements of the hidden curriculum. For a start there is less literature, and more general agreement over a definition; the null curriculum is defined as what schools (or centres) do not teach (Carpenter, 2001; Eisner, 1985; McCutcheon, 1997).

However, even here all is not straightforward. The significance of decisions about what is, or is not, taught is underlined in the literature. Such decisions reflect the realities of power and influence within a culture, the voices that have legitimacy and the voices that are silenced. As Eisner (1985) notes, the significance of: 
of options one is able to consider, the alternatives one can examine, and the perspectives from which one can view a situation or problem. (p.97)

This is a reminder that curriculum is "a cultural and, ultimately, a political act, whether we are conscious of it or not" (Provenzo, 2002, p.15).

Nor is it only content that needs to be considered. Eisner (1985) believes "the intellectual processes that schools emphasize and neglect" (p.98) are an important aspect of the null curriculum. He suggests schools tend to emphasise "a restricted conception of thinking" that is "rule-abiding" (p.98), dependent on words and numbers, and so exclude many other modes of thought. A further dimension, affect, is proposed by Flinders, Noddings and Thornton (1986), who suggest it includes such elements as values, attitudes and emotions. They believe many topics are consigned "to the null curriculum because of their potential affective impact" (p.36) and that it might be "the primary and most important single dimension of the null curriculum" (p.36). It became clear that focusing on topics that are suppressed would tell only part of the story.

Reviewing the literature relating to the null curriculum suggests other reasons why exploring the concept is challenging. The null curriculum has been described as "virtually infinite" (McCutcheon, 1997, p.189), which indicates a need to decide what might be relevant from that near-infinity of possibilities. Flinders et al. (1986) solve this problem by arguing any definition only becomes meaningful if "some universe of content perceived as educationally significant" (p.37) is used as a frame of reference, although they recognise stipulating a potential curriculum universe breaches the spirit of Eisner's original concept. However, their work highlighted dilemmas for me. The curriculum document Te whāriki does not prescribe content, and any attempt I made to limit the 'virtually infinite' to a potential universe of educationally significant content would inevitably reflect my own biases.

Flinders et al. (1986) also express reservations about the use of the null curriculum in qualitative research because of the possibility that researcher convictions colour the findings, and they consider it should not be used in experimental research, concluding it is most useful "at a meta-level in that its consideration can draw the researcher's attention to his or her own values and preferences and to those of others" (p.39). They also warn that it "cannot be defined with operational precision unless we are willing to risk triviality" (p.41). 
A further difficulty indicated by the literature, is the paucity of attention the construct has received. A search of the ProQuest Education database in June 2008 revealed 42 references to the null curriculum in document texts, but only 4 when the qualifier 'young children' was added. This indicated there was not a significant body of literature on which to build.

\subsubsection{Defining the null curriculum}

The literature outlined above had alerted me to the difficulties of defining the null curriculum, but had not removed the need to establish a working definition that would provide a starting place for this thesis. Considering definitions other than 'what schools do not teach' provided some guidance. Provenzo (2002) calls it "the curriculum that does not exist" (p.21); McCutcheon (1997) defines it as "what students do not have an opportunity to learn under the auspices of schools" (p.189). The following definition of the null curriculum was selected for this thesis:

Curriculum that arose, or potentially arose, in the setting, but that children and/or teachers deemed to be inappropriate.

This definition was broad enough to include intellectual processes (Eisner, 1985) and affect (Flinders et al., 1986) as well as content, and it acknowledged both children and teachers as actors. (Because families were only a minor part of this study it was decided not to include their perspective within the definition, although a broader study would undoubtedly have shown their influence.) This definition also required that the discussion remain grounded in the data. Inevitably, however, the parameters imposed by my ability/inability to perceive curriculum that 'arose or potentially arose' will limit the discussion.

This concludes the section on critical pedagogy; the next section addresses the sociology of childhood.

\subsection{The sociology of childhood}

The sociology of childhood is the other approach which has influenced the thesis both methodologically and philosophically. This section describes my professional route into this field, provides an overview of it, and considers how it is reflected within early childhood education. While the sociology of childhood played a significant role in the philosophical approach and the design of the thesis, this is a shorter section for two reasons. First, because one of the two ways in 
which it was influential was in reinforcing the repositioning of children as competent and worthy of research; these were ideas that I had already met within the writings of the early childhood reconceptualists and were described in Chapter 1. Secondly, reading within the sociology of childhood introduced me to a wider range of potential research strategies to use with children. However, references to this literature have been included as part of an overview of research strategies used with young children in Chapter 4. The task that remains for this section is to describe how writing in the sociology of childhood underpinned the commitment to focus on children's perspectives in the thesis.

There were three reasons for the decision to foreground the perspectives of children. First, it reflected the critical approach taken in the thesis, as it focused on the least powerful participants in the centre environment. Second, in positioning children at the centre of the research focus, it reflected a personal and professional commitment to the reconceptualising of children as actors in their own right, and worthy of study. Thirdly, it was the second of the three strategies adopted to dislocate familiar adult/early childhood teacher/teacher educator ways of thinking about teaching and learning, not least the assumptions around the power dimensions in the teacher-child relationship.

Following the decision to concentrate on the perspectives of children my reading extended into the literature of the sociology of childhood (Corsaro, 1997; James \& Prout, 1990; James, Jenks \& Prout, 1998; Jenks, 1996; Prout, 2005; Waksler, 1991; Wyness, 2006). This wider reading showed that the focus on the reconceptualisation of the child as strong and competent which was familiar within early childhood writing (e.g., Anning, 2004), particularly in relation to the Italian centres of Reggio Emilia (e.g., Edwards et al., 1994; Rinaldi, 2006) and in the reconceptualist writing (e.g., Jipson \& Johnson, 2001), was a reflection of a wider arena of discourse which had underpinned the emergence of the sociology of childhood (Prout \& James, 1990). Before this new paradigm emerged at the end of the 1980s, studies which had children and childhood as the central focus had been rare (Corsaro, 1997). Contributing factors that combined to support the emergence of the new field were: growing dissatisfaction with the developmental approach which treated childhood as a universal biological feature of human groups and the concomitant disregard for social and cultural context, awareness of childhood as a changing social construction, and a growing critique of the construct of socialisation (Prout, 2005). 
However, there was resistance to the new conception of children from both researchers and teachers. Familiar developmentally based images of the child, and the construct of socialisation both operated as 'regimes of truth' (Prout \& James, 1990). These 'regimes of truth' underpinned the two adult-held biases that Waksler (1991) identified as deflecting attention away from childhood as an appropriate focus for research—“Children are unfinished, in process, not anywhere yet" (p.63), and "Children are routinely wrong, in error, and don't understand" (p.65). But despite initial resistance, by the late 1990s, childhood had been accepted as an area of sociological research (Corsaro, 1997) and a decade later the study of children and childhood was seen to be flourishing (Broström, 2006; Wyness, 2006).

What did reading in this arena contribute to the thesis? Firstly, it reaffirmed the focus on children's lives. A central philosophical tenet of the sociology of childhood is that "children's social relationships and cultures are worthy of study in their own right, independent of the perspective and concerns of adults" (Prout \& James, 1990, p.8). Proponents recognise children as independent social actors, rather than as lesser adults, and the field of childhood sociology is seen to offer "considerable promise as a framework for further study of children and childhood, including research on education and care" (Broström, 2006, p.228).

Reading in the sociology of childhood reinforced for me that the repositioning of the child that was occurring within early childhood education was part of a wider paradigm shift. Accepting children "as active in the construction and determination of their own social lives, the lives of those around them and of the societies in which they live" (Prout \& James, 1990, p.8) was identified as a key feature of the newly emerging field back in 1990. Being familiar with the language associated with the repositioning of the child within early childhood literature enabled me to identify traces of the wider paradigm shift around children and childhood. For example, the word "competent" was familiar to me from the aspiration statement for children in Te whäriki, although there it was used with an orientation to children becoming, rather than being:

To grow up as competent and confident learners and communicators, healthy in mind, body, and spirit, secure in their sense of belonging and in the knowledge that they make a valued contribution to society. (Ministry of Education, 1996, p.9)

"Competent" was familiar from the reconceptualist literature (e.g., Cannella, 1997; Dahlberg et al., 1999), and from Malaguzzi, the first director of early 
childhood education in Reggio Emilia, whose description of the child as "active, competent and eager to engage with the world" was cited earlier (Dahlberg et al., 1999, p.7). In the sociological literature I found "competent" was again a key word used in describing how images of children and childhood had altered (Broström, 2006; Wyness, 2006). Tracing this single word suggested how the lines of discourse have spread across the boundaries of academic disciplines, and underlined for me that ideas met within early childhood education were reflections of a wider paradigm shift.

Along with validating the focus on children's lives, reading in sociology also validated the methodological approach. Ethnography has been singled out as a particularly appropriate methodology because it "allows children a more direct voice and participation in the production of sociological data than is usually possible through experimental or survey styles of research" (Prout \& James, 1990, p.8). I also found additional ideas for research strategies with young children, and in particular the work of Clark $(2004,2007)$ whose "mosaic approach" was to provide inspiration for the strategies used with children in this thesis.

There were also specific ideas that were to prove useful in clarifying my thinking in the final discussion. One was the distinction Mayall (2002) makes between positioning children as actors and as agents:

The former implies that children are of the social world: beings rather than becomings. The latter takes 'action' forward and implies that children make a difference. Children have an influence; their views are taken seriously, and there is some recognition of this within the political as well as the social sphere. (p.236)

A second was the unanticipated resonance of the writings of the sociologist Prout (2005) with Kincheloe's (2004) description of "the complexity of the lived world" (p.37), quoted above. Describing how images of children and childhood may currently be evolving, Prout (2005) writes:

...towards the end of the twentieth century there developed a pervasive sense that the social order was fragmenting under the pressure of rapid economic, social and technological change.... I will argue that childhood is also affected by this destabilization. In particular the distinction between adults and children, once firmly established as a feature of modernity, seems to be blurring. Traditional ways of representing childhood in discourse and in image no longer seemed adequate to its emerging forms.... These new representations construct children as more active, knowledgeable and socially participative than older discourses allowed. They are more difficult to manage, less biddable and hence are more troublesome and troubling (Prout, 2000a). (Prout, 2005, p.7) 
These changes, he suggests, require new approaches that move beyond ideas such as 'children are actors in their own right'. He urges for recognition that childhood is "heterogeneous, complex and emergent, and because this is so, its understanding requires a broad set of intellectual resources, an interdisciplinary approach and an open-minded process of enquiry" (Prout, 2005, p.2). This recognition of the complexity of childhood underlined the depth of challenge for this thesis.

This section has described how reading in the area of sociology of childhood contextualised and affirmed concepts that I had met in other arena, and has indicated ways in which these contributed to the data-gathering and analysis phases. The next section introduces activity theory, as the source of the three sensitising concepts.

\subsection{Activity theory as the source of sensitising concepts}

Activity theory was not an element of the theoretical or philosophical framework, but it needs to be acknowledged as the source of the three sensitising concepts. These were the physical resources and environment, the roles, and the routines, rituals and rules. In this section, following a brief outline of activity theory, the role of sensitising concepts is described and, finally, the unit of analysis is also introduced.

\subsubsection{Introduction to activity theory}

In considering possible frameworks, references to activity theory in recent New Zealand work (Alcock, 2005; Brennan, 2005; McDonald, Le, Higgins \& Podmore, 2005; Ramsey, Breen, Sturm, Lee, \& Carr, 2006) aroused my interest. Reading about activity theory as a research approach showed it 'meshed' with the scope of data to be collected for this thesis. For example, the foregrounding of the role of artefacts in activity theory paralleled the planned, but narrower, focus on the implicit messages conveyed by the resources and the physical environment. Foregrounding the constructs of roles and rules would be a way of revealing some of the hidden dimensions implicit in the ways the setting is organised, and the behaviours of the adults, and could suggest how these were affecting the boundaries of the curriculum. The decision was therefore made to adopt the nodes of the activity system model—artefacts (but only the physical resources and environment), roles, and rules_-as sensitising concepts in the thesis. 
It might be asked why activity theory was not adopted as the central theoretical framework? From the beginning it had been envisaged that an open-ended approach was most appropriate given the broad scope of the study, and the paucity of previous relevant research. While the structure of activity theory was appealing, and the option of using it was briefly considered, it seemed that adopting it as the overarching framework might impose parameters on the analysis which ran counter to the exploratory thrust. Therefore, it was decided instead, bricoleur fashion (Kincheloe \& Berry, 2004), to adopt elements from it as sensitising concepts. Using material artefacts, roles, and routines, rituals and rules_aspects of early childhood education that had rarely the focus of research-provided the third strategy of dislocation in the thesis.

Because activity theory was used only as the source of sensitising concepts in this thesis, the following discussion is intended to provide a context for understanding that use. For a fuller introduction to activity theory see Russell (2004).

Although activity theory has its origins in the work of Vygotsky, it was largely unknown outside the Soviet Union before the mid 1980s when it was taken up by Scandinavian researchers, most notably Engeström $(1987,1999)$ who extended and clarified Vygotsky's classic mediational triangle. Engeström's expansion (see Figure 2) involved adding the elements of community, rules and division of labour, which were shown as nodes relating to each other and to the other parts of the activity system. The term 'activity system' refers to a group of people who share both a common object/objective and access to a range of artefacts with which to act on that object (Russell, 2004). The rules refer to the norms and regulations that guide practices, and the division of labour describes how tasks are divided between members. The activity rather than the individual is the unit of analysis (Engeström 1987). Activity theory has been used in studying a wide range of contexts, and is particularly valued as a framework within which to consider the interplay of a range of factors. Proponents of activity theory describe it as a broad conceptual approach (Kaptelinin \& Nardi, 2000) and as "a powerful and clarifying descriptive tool rather than a strongly predictive theory" (Nardi, 1996, p.7). 


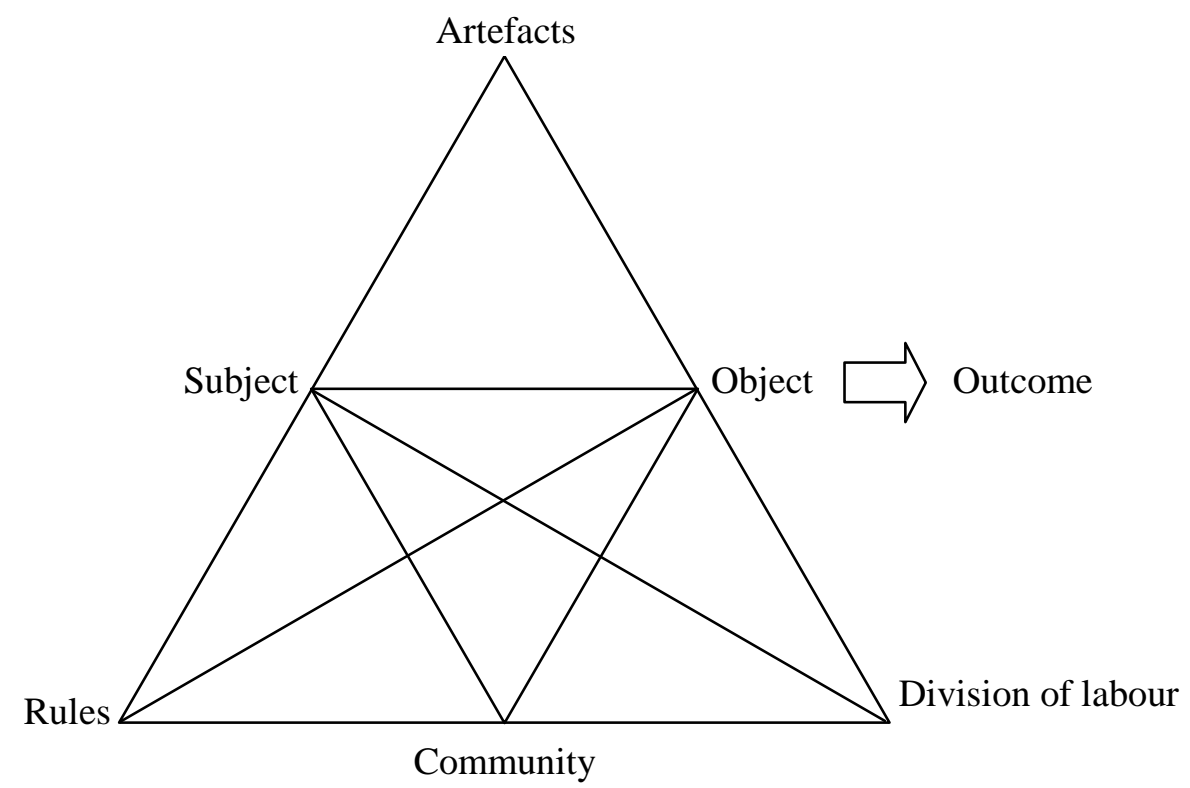

Figure 2.The structure of a human activity system (based on Engeström, 1987, p.78)

\subsubsection{The elements from activity theory used as sensitising concepts}

What are 'sensitising concepts'? Bowen (2006) provides a useful overview of the origin and use of sensitising concepts. The concept originated with Blumer and they are now used most frequently either as an interpretive device or as a starting point which reflects a researcher's first tentative analytic ideas. In this thesis they have been used in a somewhat different way; here they are used not "simply to lay the foundation for the analysis of research data" (Bowen, 2006, p.3), but also to provide thought-provoking perspectives on the data.

There are seen to be potential dangers in the use of sensitising concepts. They can become desensitising (Patton, 2002), may direct attention away from other significant details (Bowen, 2006), and should be used as "points of departure from which to study data" (Charmaz, 2003, p.259) but not as "ending points for evading it” (p.259).

\section{The first sensitising concept: The environment and the physical resources}

It is a central tenet of activity theory that activity cannot be understood without understanding the role of artefacts. Human activity is understood to be mediated by artefacts (Kaptelinin \& Nardi, 2000); artefacts reflect the experience of others who have used and perhaps modified them in the past; this experience is accumulated both in the structural properties of the artefact and in the knowledge of how to use it. Artefacts therefore shape how individuals interact with reality in 
ways that can be both enabling and limiting. They influence both external behaviour and the mental functioning of individuals, and these two processes are seen to be inseparably intertwined (Engeström 1987). Within activity theory artefacts are considered to include a wide range of physical and psychological constructs. However, within this thesis it was decided to focus only on the material artefacts - the physical resources and the environment. Disquieting references in the literature suggesting they warrant greater scrutiny provided one motivation for taking this narrow perspective. Hill (2001) describes centres as "cluttered with the little known resources and artifacts from past learning opportunities and other people's learning stories" (p.12). The following comment by Cullen (2003a) shows others share this concern:

The resources and equipment in our early childhood centres can be viewed as cultural tools that convey messages about society's expectations for young children. The same resources that have formed the "core curriculum" in New Zealand's early childhood centres for several decades can be viewed as the often unacknowledged historical dimension of Vygotsky's socio-cultural theory (Cole, 1995); that is, an expression of longstanding cultural beliefs about the nature of early education. These curriculum resources may also represent what Jordan (2002) has described as a "congealed" view of how children learn and play. (Cullen, 2003a, pp.280-281, italics in original)

A second motivation was that, although resources have rarely been the focus for research, a small-scale study by McDonald et al. (2005) had shown the insights to be gained from taking this approach. Finally, taking this focus married with the notion of the hidden curriculum, and in particular with the implicit messages that may be conveyed by the physical environment (e.g., Eisner, 1985; Jackson, 1968). Chapter 5 contains the analysis and discussion that resulted from taking this perspective.

\section{The second sensitising concept: The division of labour}

The second construct used as a sensitising concept was the division of labour, or the roles that members of the community take. Within activity theory it is recognised that roles can be explicit or implicit, may change over time, and may involve hierarchical status relationships (Russell, 2004). Within the centre, the most obvious division of labour, both in tasks and in status, was between adults and children, but over time other divisions became apparent. Adopting this as a sensitising concept through the process of analysis was to reveal unexpected aspects of both teachers' and children's roles, as the discussion in Chapter 6 will show. 


\section{The third sensitising concept: Rules, routines and rituals}

The final element from activity theory adopted as a sensitising concept within the thesis was that of rules. Norms, routines, schedules, rituals, habits, values and assumptions are all examples of rules. Here it was used to refer to rules, routines and rituals. In activity theory rules define how things should be done, and can be formal and explicit, or informal and tacit, and can alter (Russell, 2004). As Chapter 7 will show, focusing on centre routines and rituals revealed norms and values, both explicit and implicit, which influenced the boundaries of curriculum. Identifying the rules used within the centre helped to identify points of demarcation between what was designated appropriate and inappropriate curriculum.

\subsubsection{Unit of analysis}

Activity theory made one further contribution to the study; it was the source of the unit of analysis. This had been a difficulty because the intended focus of the data included both the community and the individual/s. Activity theory offered a way out of this dilemma:

The solution offered by activity theory is that a minimal meaningful context for individual actions must be included in the basic unit of analysis. This unit is called an activity. Because the context is included in the unit of analysis, the object of our research is always essentially collective even if our main interest is in individual actions. (Kutti, 1996, p.26)

Matusov (2007) warns of the danger of adopting an unmanageably large unit of analysis, and suggests Rogoff's 'planes of analysis' approach as enabling the researcher to foreground one plane while keeping the rest of the unit of analysis in the background (Matusov, 2007, p.324). In an analogous way, here the three sensitising concepts provided perspectives of analysis, while never obscuring the overall focus on the collective process of experiencing, negotiating and enacting the curriculum boundaries.

\subsection{The three strategies of dislocation}

Having introduced the sensitising concepts, the role of the three 'strategies of dislocation', which has been referred to in passing, can now be described. These were a set of unfamiliar, and sometimes uncomfortable, perspectives which I adopted in order to jar my certainties, to dislocate my assumptions and expectations, as I observed the daily activities in what was a generically very familiar context. These strategies were: 
- Focusing on what might constitute the hidden and the null curricula

- Foregrounding the perspectives of children, not only by talking with and listening to children, but also by identifying with them and thinking about how they might be experiencing the setting

- Taking a series of unfamiliar perspectives - the environment and the physical resources, the roles of teachers and children, and the routines, rituals and rules-in considering the research questions.

An underlying theme within this thesis, captured in the words of Freire (1987) quoted at the start of this chapter, is to "stimulate the certainty of never being too certain" (p.57). The discussion in this chapter has shown how that commitment to shaking my own sense of certainty influenced the choice of theoretical approach, and led to the decision to devise what I came to call strategies of dislocation. Another way in which my certainties about early childhood education might be shaken was through reading what others have written about the boundaries of curriculum, and what might constitute null curriculum. The next chapter reviews this range of literature. 


\section{CHAPTER 3}

\section{LOOSE ENDS AND LONGER THREADS: THE LITERATURE REVIEW}

Boundaries cannot be transgressed until they are made visible. (McArdle, 2005, p.90)

This literature review puts me in mind of a pulled rug, the kind where you draw lengths of wool through, knot them, and leave them as loose ends. Some parts of the review are like that, loose ends that may lead nowhere. In other places the lengths of wool are longer and wind on through the findings and discussion chapters. As I return to this chapter in the final revision stages, the question is whether to trim away those loose ends? It is tempting to do so in order to keep this chapter coherent, but on balance it seems that a responsibility of undertaking a first review of a topic is to leave those loose ends for others to peruse. Hence the title of this chapter; this review contains both the loose ends and the longer threads.

The chapter's structure is aligned with the thesis topic-how the boundaries of curriculum were experienced, influenced and enacted by children in the centre. The thread of 'curriculum experienced' opens with a discussion of how a centre's physical environment, and the embedded messages it conveys, may influence and/or limit children's curriculum experience. A range of critiques of early childhood education are introduced which are to be central to the thesis arguments.

Children's experience of curriculum is explored further in a review of literature around what constitutes appropriate/inappropriate curriculum. Literature searches showed there was no body of writing on the topic of what constitutes null curriculum for young children. It was therefore necessary to tackle the more challenging task of gathering together isolated points of discussion that broach the topic. Consequently this section of the review draws on a disparate range of professional and research literature, and includes specific challenges to curriculum detail as well as more fundamental critique of central philosophical tenets. Where it is available, New Zealand literature has been highlighted as reflecting the context in which the centre operates. Given the dearth of literature in this area, the aim has been to put a first stake in the ground in exploring where curriculum 
boundaries lie, to be indicative rather than comprehensive. The discussion is structured around the three dimensions of null curriculum proposed in the literature: content, intellectual processes (Eisner, 1985), and affect (Flinders et al., 1986); these form the second, third and fourth sections of this chapter. Outlined here this division appears clearcut, but imposing these categories on such a diverse range of writings was not always easy. In places where a topic flowed across the imposed boundaries, for the sake of coherence the material has been gathered in one place.

The chapter's final section looks at how children might influence and enact the boundaries of curriculum; here the more well-traversed literature on children's peer groups and peer culture is reviewed with a particular focus on those aspects found to be significant in this thesis.

\subsection{How might the physical environment influence the scope and boundaries of curriculum?}

Relatively little professional early childhood literature addresses issues of centre architecture and interior layout (e.g., Greenman, 2005; Olds, 2001) and even less literature contains critical reflection on these factors, yet in this thesis these were found to have significant implications for the scope of curriculum. In this section writing which raises issues in relation to the three aspects of centre location, the interior layout, and the resources provided for children, is reviewed.

\subsubsection{The physical and social isolation of centres}

The isolation of centres from the wider community is one aspect of design that has been questioned: "it is so taken for granted that children are set apart and excluded from the adult world. This setting apart occurs at community level,- - just look at the walls around old day care centres_-as well as at a theoretical level" (Singer, 1996, p.30). Writing of a New Zealand centre, Brennan (2005) concludes that "accepting the current cultural and structural arrangement at the theoretical level means that we lose sight of the fact that young children want to be part of adults' lives and included in their communities" (p.ii). As both Singer and Brennan indicate, the physical isolation of centres reflects assumptions about the place of children in adults' lives, which resonates with sociologists' recognition that children today are typically "sited, insulated and distanced" (James et al., 1998, p.37) from adults' social space. Underlying this is a belief that children learn, 
think and feel in ways that are different to adults (Singer, 1996). Singer, like Cannella and Viruru (2004), links the separation of children from adults' lives to the 'child-centred' approach of early childhood education. On the one hand this directs:

...attention to the specific requirements and needs of children, which is a good thing. But, this way of thinking also legitimises the exclusion of children from the adult world: they are not allowed to be part of it, they must be child-like; they cannot yet think logically; their judgement is 'cute and charming', but cannot be taken seriously. This impedes their learning and is belittling them. (Singer, 1996, p.30)

Singer (1996) sees "social emptiness" (p.31) and monotony as perils of such separation and notes it contradicts the self-proclaimed sociocultural focus of early childhood education which envisages children learning through participating in the activities of those who are more skilled. Brennan (2005) suggests "the physical structure results in a rather contrived social situation that teaches children how to survive childcare but may have little to do with other aspects of their cultural life" (p.213).

Descriptions of other models of early childhood education, both internationally and culturally, that are founded on different visions of adult-child relationships, offer other possibilities. For example, the centres of Reggio Emilia in Italy are "situated in highly visible settings within the city to permit children and teachers to contribute to the daily activity of the community, and to allow the schools to be appreciated as vital, dynamic aspects of city life" (Goffin \& Wilson, 2001, p.235); community participation is encouraged and the boundary of the learning environment is seen to extend well into the community (Fleer, 2003a). Wright's (2003) description of home-based environments extending out from the home and into the community is a similar New Zealand example. Writing philosophically rather than architecturally, Dahlberg and Moss (2005) describe their vision for early childhood as "environments where the coming together of children and adults, the being and thinking beside each other, offers many possibilitiescultural and social, but also economic, political, ethical, aesthetic, physical" (p. 28). This line of commentary, stemming from the physical location of centre buildings, was the starting point for a central theme within the thesis, which would both reinforce and extend the critique outlined above. 


\subsubsection{The interior design and layout}

Teachers create environments that reflect their beliefs about children and education. Thirty years ago, in the United Kingdom, King (1978) wrote "Children were defined as being naturally interested in things: in consequence the classroom was set up to evoke their interest. Children were defined as having individual propensities for learning: in consequence a wide range of educational material was provided" (p.18). More recently Skinner, Bryant, Coffman and Campbell (1998) report North American teachers' classroom environments reflect their belief that children are capable of self control.

However, the ways in which a centre's interior design can influence children's experience of curriculum has received limited attention. For example, the open layout in a single room, typical of purpose-built centres in New Zealand, is seen to allow children's freedom of movement (Anning, 1991), and freedom to choose activities and resources (Walsh, 1996) thus promoting children's independence (Olds, 2001; Walsh, 1996), although it is recognised such space needs to be subdivided so children do not run around "aimlessly" (Walsh, 1996, p.18). But others question how much freedom of movement children truly have, suggesting the apparent freedom open layouts convey is illusory. Rather, children have to learn the rules governing the use and possibilities of that space (Bernstein, 1975/1997; Cannella \& Viruru, 2002). Cannella and Viruru (2002) suggest the appearance of freedom is a strategy designed to control; Lofdahl (2002) links it to the hidden power structures Foucault described. This notion of illusory freedom will be returned to in discussing children's access to spaces and resources within the centre.

The provision of private spaces for children is an aspect of design that has opened up philosophical issues around supervision and surveillance. Children are seen to need access to withdrawal spaces (Greenman, 2007; Olds, 2001; Prescott, 2008). Where children are cared for in a single large room, which is often the case in New Zealand, limited withdrawal spaces mean children must find other ways of retreating (Test, 2006); Greenman (2007) suggests children do this by learning to filter out contact. However, in some European settings children are not only able to withdraw, but they can also be apart from adults; there are descriptions of Icelandic children playing alone in rooms with the door closed (Einarsdottir, 2006), of Swedish toddlers playing alone in a room (Test, 2006), and of children playing out-of-sight of teachers in forest areas (Maynard, 2007). 
The lack of provision for children's withdrawal is linked to assumptions about supervision: "unfortunately in the United States we have almost entirely defined supervision as surveillance, rather than a combination of carefully planned environments, and established norms and expectations for behavior. Privacy and seclusion are considered not only unnecessary, but inappropriate" (Greenman, 2007, p.23, italics in original). Nimmo (2008) suggests the discourse of risk provides added legitimation for children's surveillance, making even trips difficult because of the consuming concerns about safety. Such dwindling access to, and participation in, real life beyond the centre has been seen as symptomatic of the growing boundary between the worlds of adults and children (Singer, 1996).

Assumptions around the supervision of children are challenged, particularly by reconceptualist scholars. Cannella and Viruru (2004) argue that adults' surveillance of children is legitimised by theories of child development and education. The supremacy of the teacher, holding a powerful position of protection in a carefully planned, safe environment, is constructed on the image of the child as innocent, an image which Woodrow and Brennan (2001) suggest underpins the traditions of early childhood pedagogy. Cross-cultural comparisons indicate such perceptions of children are a particular feature of Western culture; for example, Dockett and Fleer (1999) describe Australian Aboriginal communities allowing children far more control in their lives.

However, teachers themselves are also under observation. The New Zealand requirement that every centre have an abuse prevention policy which ensures adults are visible to other adults whenever they are with children came in the aftermath of a high-profile sexual abuse case (Duncan, 1999). While these guidelines were promoted as keeping both children and adults 'safe', Duncan (1999) links the changed attitudes and behaviours of teachers to Foucault's notion of surveillance, and comments that teachers unwittingly "created a surveillance regime that almost replaces the law in effectiveness" (p.251).

Although only a limited pool of literature was located that considers how centre architecture might influence the scope of curriculum, it nevertheless raises disquieting questions. Would a review of literature about the provisioning of centres reveal similar concerns? 


\subsubsection{The provision of resources}

Much professional literature on the topic of resources focuses on preparing environments for children, reflecting a belief that children learn by doing (Anning, 1991). However, positioning the teacher as "the magic facilitator, preparing the right environment through which development will unfold" (Woodrow \& Brennan, 2001, p.30) is linked to the image of the child as embryo adult, moving from irrationality to rationality following a preordained sequence (Woodrow \& Brennan, 2001). Despite New Zealand teachers' professed commitment to the sociocultural approach of Te whäriki, Hill (2003) notes there is still a "prevalence of adult preoccupation with the environment, the programme and selected resources (covertly tied to Piagetian theory) at the expense of interaction with children" (p.25). Two aspects of resourcing are of particular interest. The type of resources opens up for discussion the assumptions embedded in the statement that play is the child's work. The quantity of resources and the way in which they are displayed leads into discussion of the balance between children's freedom of choice and teachers' control.

The often unquestioning commitment to the traditional resources of early childhood education, such as blocks and playdough, is an area of discussion both in New Zealand (Cullen, 2003a; Hill, 2001) and internationally. Cuffaro (1995) urges American teachers to reflect more deeply on their choice of resources; if they are selected ""because they are part of the early childhood curriculum" or simply "because children enjoy the activity," then thinking remains on the surface, on acceptance of conventions that remain unexamined" (p.33-34). Australian scholar Fleer (2003a) sees reassessing traditional play areas as a step towards reconceptualising early childhood education. While it is beyond the scope of this thesis to describe the origins of the traditional resources within early childhood education, these are well documented (Williams \& Fromberg, 1992; Roopnarine \& Johnson, 2005). The current New Zealand list of recommended equipment (Ministry of Education, 2004a) demonstrates the continued commitment to such provision.

Descriptions of resources used in other models of early childhood education highlight the assumptions about adults' and children's roles that are implicit in definitions of appropriate/inappropriate. Children in an English Forest School use adult-size bow-saws and loppers, and cook over an open fire (Maynard, 2007). 
Israeli children play in junkyards stocked with cast-off farm machinery and building equipment (Haas, 1996). In some New Zealand centres children use handsaws and battery-driven drills in constructing gates and furniture (Carr, 1998). In the centres of Reggio Emilia children can use 'adult' resources (Fleer, 2003a; Gandini, Hill, Cadwell \& Schwall, 2005).

Cannella and Viruru (2004) suggest the concepts of the child as player and of play as the child's route to learning underpin the provision of traditional play resources. These concepts have long been central tenets of early childhood education both in New Zealand (Grey, 1974) and internationally (Paley, 2004; Wood, 2004). But these tenets have recently been challenged. Defining play as the normal behaviour of childhood and as necessary for growth and development is challenged by Cannella and Viruru (2004) using historical and cultural examples. Assuming the normality of play is seen to privilege learning by doing (Cannella, 1997; Fleer 2003a) and to imply that success depends on having enough money to provide play resources (Cannella, 1997). Even the apparent freedom of play is seen to be false, with adults defining what constitutes 'good' and 'bad' play (Cannella, 1997). Most significantly for this thesis, the discourse of play has also been challenged as reinforcing "the marginalization and exclusion of children from society, and thus from influence in real life" (Strandell, 2000, p.147). Positioning play as opposite to work is seen to trivialise play and ignore the reality of the social and power relations within it (Ailwood, 2003). Ailwood (2003) suggests play has become so central to early childhood education that it "governs the behaviour of both adults and young children" (p. 286). These challenges to the centrality of play have been reiterated by the New Zealand scholar Gibbons (2007). Has the discourse of play become a regime of truth, unrecognised but influential, that limits the ways in which we envisage early childhood education? This thesis, grounded in the day-to-day activities of a centre, was to provide some corroboration for these philosophical critiques.

A number of points of discussion impinge on the question of how resources influence children's experience of curriculum, and draw particular attention to the (often implicit) dimensions of teacher control. The quantity of accessible resources has drawn comment (Skinner et al., 1998) with words such as "enormous" (King, 1978, p.17) used to describe the range. On the one hand it is suggested that a vast array may be overwhelming for children (Anning, 1991), but 
on the other environments are valued for the wealth of opportunities they offer (Laevers, 2005). Test's (2006) comparison of infant toddler centres in the United States and Sweden provides insight into the cultural dimensions of provisioning.

Earlier writers stressed the level of teacher control over the provision, definition and use of resources (Apple \& King, 1977; King, 1978), and the need for children to distinguish between resources for work and play (Apple \& King, 1977; King, 1978). Apple and King (1977) suggested such learning was a first step in assuming the role of student. More recent accounts reveal a similar level of teacher control of resources (McCadden, 1998; Skinner et al., 1998) which often extends to a prohibition on children bringing resources from home (Corsaro, 1985, 2003).

While accessibility of equipment is typically linked to children's freedom and autonomy (Walsh, 1996), the promotion of these qualities is challenged by Alloway (1997) on the grounds that they represent cultural and gendered ideas of what it means to be emotionally mature. It is also argued that the freedom implied by accessible equipment is in fact illusory, that "adults actually control the choices that surround children" (Cannella \& Viruru, 1997, p.121) and children are governed through that very pretence of freedom. Teachers' control of resources offers another avenue by which they control the definition of acceptable play; King (1978) cites the familiar example of a prohibition against making guns.

Considering this range of literature on the resources provided for children suggests this is an area that requires further scrutiny, particularly in relation to covert messages that may be implied in the kind of resources provided, and the ways in which children may use them. The most far-reaching challenges (Ailwood, 2003; Alloway, 1997; Cannella, 1997; Cannella \& Viruru, 2004) have been raised in theoretical writing. This thesis will show that similar issues surfaced in observing the day-to-day use of resources in a centre. The review of these three areas of literature relating to the physical location of the centre, the interior layout and the resources for children provides the context for Chapter 5, in which the impact of the centre's environment on children's experience of curriculum is described. 
In the following three sections literature relevant to the concept of the null curriculum is reviewed. References to null curriculum in research literature are rare, and while the phrase has been used occasionally in New Zealand work (Hedges \& Cullen, 2005; Podmore, Sauvao \& Mapa, 2003) it has not been the focus of research. This might indicate acceptance of the status quo but, as the following review will show, a number of current areas of debate impinge on the topic. The demarcation between appropriate and inappropriate curriculum is considered in relation to content first, and then to intellectual processes (Eisner, 1985), and finally affect (Flinders et al., 1986).

\subsection{What content might be marginalised or considered null curriculum?}

The question of curriculum content within early childhood education has not been a recent focus; Goffin and Wilson (2001) suggest "deliberations regarding what knowledge is of most worth" (p.195) have been sidelined in favour of comparing curriculum models. However, children's unprecedented access to information and the consequent blurring of traditional distinctions between adult knowledge and child knowledge (Kincheloe, 2002) may alter this. Recognising such a change is occurring underlines the timeliness of this thesis.

Where debates about the scope of curriculum content do occur, they tend to concern single issues, points on the border between what is deemed appropriate/inappropriate. Some scholars challenge the exclusion of particular content, others describe successful inclusion of content; both approaches indicate an area under debate. What are the points of debate? Are there common themes? These are the topics for the following sections.

\subsection{1 'Real' tasks as curriculum?}

Children's exclusion from work, from 'real' tasks has been challenged as being a reflection of the work/play dichotomy which Cannella and Viruru (2004) believe perpetuates the separation of child and adult, and positions children as players. Nimmo (2008) links the absence of real jobs to children's exclusion from the world of adults, and to the concept of children as incomplete. Descriptions of young children engaging in work in other cultures (Rogoff, 2003) and descriptions of children doing real tasks in centres (Drummond, 1999; Nimmo, 2008) such as shovelling snow and digging gardens (Jones, 2005) further unsettle assumptions. 
Debates about the place of ICT (Information and Communication Technology) in curriculum can be considered, in part, as an extension of the debate about the place of real tasks. While most contemporary professional literature is broadly supportive of young children's use of ICT (Bolstad, 2004; Stephen \& Plowman, 2002) negative arguments have been mounted particularly concerning computers.

In the New Zealand context, alongside cautions about their use with young children (Brownlee, 2000), there are descriptions of children using ICT extensively and successfully (Ramsey, Sturm, Breen, Lee \& Carr, 2007). Bolstad (2004), having reviewed both New Zealand and international literature, concludes ICT “can be a useful tool for supporting children's learning and development" (p.72). Yet in many New Zealand centres (Oldridge, 2009) teachers have access to a computer but children do not. Are decisions about who uses a centre's computer symptomatic of deeper philosophical assumptions? Considering what real tasks were available for children, and what 'adult' equipment they could access proved to be useful leads to follow in data generation in the centre.

\subsubsection{Are death and disaster appropriate curriculum content?}

Some topics have conventionally been labelled inappropriate for children (Cannella, 1999). Death (King, 1978; Silin, 1995; Woodrow, 1999), AIDS (Silin, 1995) and major tragedies such as the Twin Towers September 11 event (Connerton \& Patterson, 2006) have all been identified as traditionally avoided topics, reflecting the positioning of children as innocent and needing protection. However, shielding children from such topics has been challenged as denying children the chance to explore issues that impact on them (New, Mardell, \& Robinson, 2005; Silin, 1995, 1997). Silin (1995) argues teachers have a pedagogical responsibility to address such topics and place children's "questions and concerns at the center of the curriculum" (p. 46). The underlying concepts of child and teacher implicit in such exclusions have been questioned. Silin (1995) challenges the role of teacher-as-protector, pointing out how closely aligned innocence is to ignorance. Roberston (2006) asks teachers to consider whether their image of children really sees them as "grappling with and thinking about difficult and uncomfortable topics" (p.44).

In North America the graphic television footage of the Twin Towers event catapulted many teachers into confronting issues of death and disaster with children, and descriptions of how they handled this (Connerton \& Patterson, 2006; 
Gross \& Clemens, 2002), and other episodes of death (New et al., 2005), underline how rarely such topics are part of curriculum. However, those descriptions also suggest children's readiness to engage: "The children knew we had tapped into something really powerful, something that school and adults don't often let us tap into, a way of exploring our fears and the things we don't understand" (Paley, 2004, pp.108-9). Teachers' avoidance of death is further challenged by the fact that dying is a frequent theme in children's play (Corsaro, 1985, 2003; Paley, 1984). In the New Zealand context, Brennan (2005) found children "repeatedly asked teachers about significant life events such as death, dying, illness and tragedy" (p.185). This accumulated evidence suggested death might be a contested curriculum topic.

\subsubsection{Are the body and sexuality appropriate curriculum content?}

The sociologist Prout (2005) suggests the body has come to be excluded from social science, despite children's intense interest in their own bodies. Five aspects are seen to have particular significance for children-height, shape, appearance, gender and performance (James et al., 1998, p. 155). American scholar, hooks (1994), argues that the accepted notion of mind-body dualism, and the concern of education with the mind, has led to the erasing of the body from educational contexts. Has the body also been excluded from young children's curriculum? Sanson $(2006,2007)$, writing about dance in New Zealand centres, suggests the body has been increasingly excluded, and this thesis will confirm that view.

More writers address the allied topic of sexuality within early childhood (Blaise \& Andrew, 2005; Robinson, 2005). Although Tobin (1997) considers the exclusion of sexuality has emerged since the 1950s, Isaacs (1933) was addressing similar issues in the 1930s in England. Generational assumptions have been linked to the debate: Robinson (2005) sees the silencing of sexuality as yet another expression of the adult-child binary. She argues it is the intersection of the discourses of childhood and sexuality, together with the psychological discourses of child development, that have led to sexuality being constructed as taboo. Rejecting definitions of sexuality as physical sexual acts, Robinson calls for a broader understanding of it as being about aspects of everyday life such as "relationships, life choices and practices, dispositions, pleasures, desires and fantasies" (p.22). A particular aspect of the debate relates to the portrayed models of sexuality. Defining heterosexuality as the norm and not reflecting diverse identities and 
family types is challenged (Robinson, 2005; Ryan \& Grieshaber, 2004; Skattebol $\&$ Ferfolja, 2007). Robinson (2005) argues teachers' unthinking acceptance of activities such as mother-and-father play is part of the normalisation of heterosexual desire, and that the discourse of childhood and the tenets of developmental theory act to conceal this process. New Zealand scholarly literature on the topic of sexuality as curriculum reflects elements of the wider international discussion. Surtees (2003) labels the lack of acknowledgement of sexuality in $T e$ whäriki a form of censorship, and the implicit acceptance of heterosexuality as the norm is challenged (Gunn, 2006; Lee, 2005; Surtees, 2003).

There are calls for change; for allowing topics such as the body and sexuality to surface (Blaise \& Andrew, 2005). Rinaldi (2006) acknowledges it requires confronting "our taboos, silences, hypocrisies, fears, about our real feelings and emotions regarding children—our children—and ourselves" (p. 93) but sees change as necessary if teachers are to help children accept and appreciate their own bodies. However, it is acknowledged parents may consider sexuality, perhaps along with spiritual, religious and cultural values, to be topics which lie outside the teacher's role (Alloway, 1997).

The conventional exclusion of these topics — death, disaster, sexuality-from the curriculum reflects traditionally accepted boundaries between adults' and children's knowledges. But there are other topics, less clearly defined, which also impact on children's lives, and which teachers have typically avoided as 'too hard' for children. These are topics around issues of social equity and justice including gender.

\subsubsection{Issues of social equity and justice as curriculum?}

Calls for teachers to take a proactive stance with children on issues of social equity and social justice (MacNaughton, 2003b; Rhedding-Jones, 2005;

Roberston, 2006; Ryan \& Ochsner, 1999; Silin, 1995; Woodrow, 2003) indicate this is an area of curriculum debate. "Ignoring the hard stuff and only engaging in the fluff and fun from curriculum choices is to keep underground issues of social justice and to further silence and compound the inequity" (Robertson, 2006, p.45). Children are seen as competent to reflect on such issues and to understand their own role in enacting or confronting social injustice (MacNaughton, 2003b). Silin (1995) argues when teachers fail to take a proactive stance they are in danger of 
reinforcing the stereotypes, but acknowledges the challenge: "the difficulty of fostering open dialogues, acknowledging uncertainty, and respecting multiple perspectives cannot be minimized" (p. 128). However, there are also warnings. While Rhedding-Jones (2005) calls for teachers to "prepare both children and adults to become change agents via challenging stereotypes and norms of behavior" (p.144), she warns that the label of "diversity is 'loaded' with complexities, innuendoes and omissions" (p.144). For example, PaciniKetchabaw (2007) shows how the apparently benign discourses of multiculturalism have been used unintentionally to disadvantage immigrant children.

Confronting children's gendered behaviours and thinking draws particular comment (Fleer, 1998; Hughes \& MacNaughton, 2001; Keddie, 2003;

MacNaughton, 2000, 2001). Scholars identify how discourses of early childhood can act to frame and limit teachers' practices around gender. In particular, if teachers' practices are underpinned by a discourse of children as innocent, they are unlikely to confront gendered issues of violence and aggression (Grieshaber, 2001; Keddie, 2003). Strategies such as modelling anti-bias behaviours (Sims, 2000) or providing equal opportunities are seen to be insufficient responses (Browne, 2004; MacNaughton, 2001) and, unwittingly, teachers may even contribute to gender stereotypes (Martin, 1998). Rather, teachers are encouraged to adopt proactive strategies such as discussing gender issues with children, exposing them to different models of masculinity/femininity, and challenging their stereotyped responses (MacNaughton, 2000). Australian scholars (e.g., Fleer, 1998; MacNaughton, 2001; Keddie, 2003) and American scholars (e.g., Grieshaber, 2001; Ryan \& Ochsner, 1999; Silin, 1995) have been to the fore in raising these issues. There has been less focus on issues of social justice by New Zealand scholars and researchers (Ritchie, 2005), and less recent focus on gender issues (Norris, 2001), so there was little to suggest how teachers in the centre might respond to issues of social justice and gender stereotyping if and when they arose.

The discussion so far has considered potential curriculum topics teachers may exclude as inappropriate. But there is also literature that describes content children may introduce which teachers may contest. 


\subsubsection{Which interests are accepted as curriculum?}

King (1978) described English teachers giving educational status to some children's interests and ignoring others, such as popular songs, comics, television programmes, and "anything to do with fighting, death or wars, unless these were safely set in a historical context” (p.39). Reports of teachers' differential responses to children's interests, or pre-existing funds of knowledge, continue to appear. Hedges (2007) suggests that New Zealand teachers' marginalising of some interests may reflect limited teacher-family communication, plus the dominance of some children in interactions. Nuttall (2004) found the demands of routines and maintaining children's compliance led to children's interests being silenced. In an Australian setting it was suggested teachers' values influenced who they interacted with (Fleer \& Robbins, 2007); the interests of children whose thinking was similar to theirs, who spoke English, and who played quietly were more likely to be privileged.

Can children's desire to have friendships and to be accepted by peers be considered an interest? This question foreshadows the discussion of children's enjoyment of peer interactions in section 3.5.1 below. The more specific question to be addressed here is do teachers recognise children's peer relationships as curriculum? Commonsense suggests they do, indeed there is a wide range of literature on children's social skill development in professional journals. Nevertheless, the teaching of social skills has been identified as an excluded curriculum area. Graue (2005) found teachers rarely taught social skills, but rather tended to reiterate rules and repeat the mantra 'use your words'. She hypothesises this may reflect a belief that social development is a pattern of maturation but argues that "in a powerful inversion of the normative model, eligibility for participation is limited to those who already have the desired attributes" (p.48). Graue's comments resonate with McCadden's (1998) conclusion that it is inappropriate to leave children to cope with the challenges of learning social interaction skills: "over time, defeats for the children who had trouble fitting in or advocating for themselves grew larger and larger, bringing with them their own negative intersubjective understanding" (p.89). His conclusions led him to introduce a teaching programme about friendships. It seemed likely that I would find friendships were an interest for children, but the role teachers might take in supporting these seemed less certain. 
There is also acknowledgement in the professional literature that teachers need to make choices amongst children's interests (Edwards et al., 1994; Fraser \& Gestwicki, 2000). For example, Jones and Nimmo (1994) indicate much of children's peer culture "lies outside the limits that teachers impose for their classrooms-spitting, swearing, fighting, and maybe Barbies and war toys" (p.45).

The reference to Barbies and war toys raises an issue that has been called "complex, contested and contradictory" (Giugni, 2006, p. 222), the place of children's popular culture in early childhood education. Popular culture is frequently cited as an area of children's knowledge that is excluded (Fleet, 2002; Giugni, 2006; Jones \& Nimmo, 1994; King, 1978; Ryan \& Grieshaber, 2004) with varying reasons offered. For example, there is a perception that children playing with the products and concepts of popular culture are not learning, concern about the stereotyped images portrayed (Arthur, 2001), and about the impact of commercialisation on children (Aird, 2004; Nimmo, 2008). Despite teachers' reservations, children saturate classrooms with images of popular culture on their clothing and personal possessions (Hadley \& Nenga, 2004), and there is evidence of children's depth of knowledge around such concepts and products, and their use of this knowledge in their play (Arthur, 2001; Hadley \& Nenga, 2004), which New Zealand scholars reiterate (Brennan, 2001, 2002; Watson, 2005). Children's use of images, characters and plots from popular culture has also been seen as a way in which they separate themselves from adults (Thompson, 2003). Excluding such play has been called an issue of social justice because popular culture provides children with access to a common script and therefore allows all to participate on an equal footing regardless of social, economic, racial or language background (Arthur, 2001; Ashton, 2005). (The role of popular culture in children's gendered discourses is referred to in section 3.5.5 below). While the many references to children using elements of popular culture in their play, and to teachers' rejection of these, suggested this might also be a contested curriculum topic in the centre, this was not to be the case.

There are other debated areas of curriculum content, such as the place of academic subjects (Katz, 2007; Laevers, 2005) and the question of whether teachers have adequate content knowledge (Cullen, 1999; Garbett, 2004; Hedges \& Cullen, 2005). However, these are not areas of content that children influence; rather the 
debate tends to be one that occurs between adults, between teachers, parents, teacher educators, researchers and policy-makers, so these are not included.

Reviewing these areas of literature was fruitful in suggesting possible leads. While initially the review appeared to cover a disparate array of topics, a theme of generational assumptions and a positioning of children as less competent underpinned many of the debates. These were also to be significant in my own analysis.

The following section considers ways in which aspects of thinking may be privileged/marginalised within early childhood education.

\subsection{Which ways of thinking might be marginalised or considered null curriculum?}

Eisner (1985) was the first to label intellectual processes an aspect of null curriculum, and he suggested schools emphasised logical thinking that was dependent on words and numbers. In consulting the literature that addresses young children's intellectual processes, the particular focus has been on identifying those aspects which scholars suggest may be either marginalised or privileged within early childhood education.

The call to reconceptualise children as thoughtful people (Cannella, 1997; Laevers, 2005; Rinaldi, 2006) provides the starting point. Laevers (2005) writes that we need to "take children seriously and see them as partners, as active coconstructers and promoters of their own learning" (p.22). The impassioned tone of writing on this topic by Laevers and others suggests positioning children as thoughtful and competent is an ongoing debate. As was suggested in Chapter 1, although New Zealand children have increasingly been included in assessment and in research, there is evidence (Bernstone, 2007; Jordan, 2003, 2004; Nuttall, 2004) suggesting teachers may still be grappling with the reconceptualisation of children as competent and thoughtful.

\subsubsection{Do cultural assumptions influence the privileging/marginalising of ways of thinking?}

Reconceptualist scholars draw attention to ways in which cultural assumptions within early childhood education may privilege/marginalise some children. The assumed dominance of western/individualist perspectives within early childhood 
education is increasingly questioned (e.g., Fleer, 2003a; Yelland \& Kilderry, 2005). New Zealand scholars Ritchie (2001) and Lee (2005) both acknowledge the tension for teachers. Lee writes that they "walk a fine line between the expectations on them as educators: promoting individual accomplishment from a eurocentric ... success orientated perspective on one hand, and demonstrating respect for a more collectivist, group focused approach on the other" ( p.61). Ritchie (2001) believes a shift is needed from a traditional emphasis on "fostering independence and autonomy" (p.139), and that teachers should also foster "children's social skills and collective endeavour" (p.137). Writing more recently, in reference to both Australia and New Zealand, Farquhar and Fleer (2007) indicate such fundamental changes are slow to occur:

We have not as yet seriously disrupted the western developmental perspectives as the main and only view of early childhood education. We have also not taken account of the linguistic and interactional patterns which feature among many cultures outside of the western world. (p.42)

A number of particular culturally based practices are seen to impinge on the kinds of thinking and learning promoted. These are the privileging of language as the dominant means of communication (Fleer \& Robbins, 2004; Viruru, 2001), the subsequent marginalising of other ways of learning such as observation (Fleer, 2003; Rogoff, 2003; Rosenthal, 2003) and the privileging of teacher questioning (Fleer, 2003a, 2006).

Professional literature addressing multiculturalism, and the more specific notion that every child's culture and language should be reflected in the environment (e.g., Gonzalez-Mena, 1997), is also relevant to the kinds of thinking which may be privileged/marginalised. There is recognition multiculturalism can be, at worst, superficial, uncritical and reinforce assimilation (McLaren, 1994). Similar understandings are evident in early childhood literature. For example, teachers' professed commitment to acknowledging a child's home language may not challenge assumptions around use of the dominant language (Viruru, 2001) or dominant ways of thinking. There are, on the other hand, descriptions of what cultural diversity may offer (e.g., Laevers, 2005) and a New Zealand account records how a sense of belonging for Samoan children was nurtured through bilingual and bicultural practices, and that the acknowledgement of different cultural values enhanced learning for all children (Simmons, Schimanski, McGarva, Haworth \& Cullen, 2007). 
Within New Zealand, biculturalism provides a special dimension to this discussion of privileged/marginalised ways of thinking and learning. The commitment to biculturalism derives from the Treaty of Waitangi, signed in 1840, which established the notion of partnership between the Crown and the indigenous Māori. A commitment to biculturalism is integral to Te whāriki and widely accepted through the sector (Ritchie, 2003). However, there are also indications that the vision of the curriculum document may not be reflected in practice. For example, Lee (2005) reports "much of te reo Māori used is only in the form of commands. This means that children learn, not that te reo Māori is a vital and rich language, but that it is a language in which you are told what to do" (p.59). More fundamentally Ritchie (2005) challenges non-Māori teachers to confront the power relations that have simultaneously historically positioned them as curriculum experts and marginalised indigenous cultural knowledge, and warns of the danger of reinforcing culturally bound positions. These concerns, combined with the more general concerns raised in the previous paragraph, suggest that even in New Zealand, where there is commitment to a bicultural curriculum document, observations in a centre may show that the English language, and western world, individualistic ways of thinking are promoted.

\subsubsection{Are there other ways of thinking that might be privileged/marginalised?}

Children's thinking has attracted recent research attention both internationally (Epstein, 2003; Robson, 2006; Robson \& Hargreaves, 2005), and in New Zealand (e.g., Bernstone, 2007; Hedges, 2007). A central conclusion Siegler and Alibali (2004) reach, on the basis of an extensive review of research, is that young children "have a much richer cognitive life than was suspected until recently" (p.435) and that differences between adults' and children's thinking are not as large as previously imagined. Given this finding, how can one identify which ways of thinking might be null curriculum? The 16 habits of mind identified by Costa and Kallick (2000) as being significant ways of thinking in intelligent behaviour provide a touchstone for this discussion. While the authors caution that other habits may be added, this list provides a useful framework against which to consider the points made in the early childhood literature:

- Persisting

- Managing impulsivity

- Listening with understanding and empathy

- Thinking flexibly

- Thinking about thinking (metacognition)

- Striving for accuracy 
- Questioning and posing problems

- Applying past knowledge to new situations

- Thinking and communicating with clarity and precision

- Gathering data through all senses

- Creating, imagining, innovating

- Responding with wonderment and awe

- Taking responsible risks

- Finding humour

- Thinking interdependently

- Remaining open to continuous learning

(Costa \& Kallick, 2000, p.xiii)

Studies suggest adults tend to underestimate the range and subtlety of young children's thinking (Siegler \& Alibali, 2004), and that teachers are also culpable. For example, in a small-scale English study, teachers talked about strategies for supporting children's thinking as "'teaching thinking', 'teaching thinking skills', or even 'teaching children to think', as if, somehow, they were not doing so already" (Robson, 2006, p.161). There are also indications that teachers do not consider in detail how they might most effectively support children's thinking (Robson \& Hargreaves, 2005), and tend to conflate thinking skills with problem-solving (Robson, 2006; Robson \& Hargreaves, 2005). Would the findings in this thesis suggest the situation in New Zealand is different?

Suggestions of what may currently be overlooked aspects of young children's thinking indicate three areas of interest. The first relates to the disposition one has as a thinker (Claxton, 1999; Robson, 2006). The other two relate to potentially marginalised ways of thinking: thinking creatively (Claxton, 1999; Robson, 2006; Robson \& Hargreaves, 2005) and thinking critically (Robson, 2006). Robson (2006) indicates creative thinking combines originality and imagination, and is characterised by openness to challenge, risk and uncertainty, and enjoyment of complexity. Critical thinking combines learning how, when and what to question, and how and when to reason. However, creative and critical thinking are seen to be interdependent — children use both as they play, and problem-solving requires both (Robson, 2006).

\subsubsection{Dispositions as a thinker as curriculum?}

While learning dispositions are not synonymous with the disposition to be a thinker, these provide a useful starting point. Learning dispositions are the primary focus of the learning stories which are the currently promoted assessment approach within New Zealand early childhood education (Carr, 2001; Ministry of Education, 2004b). 
Learning dispositions have been described as "situated learning strategies plus motivation-participation repertoires from which a learner recognises, selects, edits, responds to, resists, searches for and constructs learning opportunities" (Carr, 2001, p.21). The five dispositions identified within New Zealand assessment processes are taking an interest, being involved, persisting with difficulty, expressing an idea or a feeling, and taking responsibility or taking another point of view (Carr, 2001). A focus on dispositions in assessment of young children is not restricted to New Zealand; Laevers (2005) reports a range of dispositions, such as creativity, selforganisation, self confidence and resilience, have been getting more attention internationally in early childhood curricula. Resourcefulness (Claxton, 2002) and playfulness and reciprocity (Carr \& Claxton, 2004) have also been proposed. Recently, a New Zealand study has used resilience, reciprocity and imagination as three "domains of learning dispositions" (Duncan, Jones \& Carr, 2008).

The notion of learning dispositions has been critiqued as a discourse that seeks to govern the child, just as the discourse of child development did, as reinforcing traditional notions of the child as able to be 'read' by the adult, and as assuming there is universal agreement on what is normal and desirable (Campbell, 1999). However, for this review what is more pertinent is how such a framework prioritises or diverts attention from different ways of thinking. While it is clear that skills and knowledge are level one outcomes within the four-level conceptual hierarchy of increasing complexity inherent in learning dispositions (Carr, 2001), the question remains of whether New Zealand teachers are aware of, and address in their teaching, a wide conception of thinking processes such as those listed by Costa and Kallick (2000). No literature was found addressing that issue. The rest of this section, drawing on a range of literature, will go only a little way towards answering that question.

\subsubsection{Thinking creatively as curriculum?}

Thinking creatively (Robson, 2006), imagination and creativity (Robson \& Hargreaves, 2005) are all identified as potentially overlooked aspects of thinking in early childhood education. There is a range of aspects to thinking creatively. For example, divergent thinking (Crook, 2004) — the ability to think of new ways to use a familiar object, to improvise — and humour (Loizou, 2005) are both seen as aspects of thinking creatively. My expectation was that these ways of thinking were well supported in New Zealand centres, but this thesis is about ignoring expectations, and so I read further. 
Both the environment and the pedagogical approach are seen as important in supporting children's creative thinking. Crook (2004) notes divergent thinking is supported by the provision of open-ended materials, improvised play materials, and adults who encourage children playing around with ideas and materials and value exploring as much as problem-solving. Laevers (2005) indicates two qualities of the environment need to be considered. One is the 'richness' and potential for challenge, the second is the scope the environment offers children for re-presenting. A rich environment is "a context that challenges children and offers a myriad of opportunities to be active, explore and create" (p.22), and that is sufficiently complex to allow all children the "joy of discovery" (p.22). The atelier or workshop of Reggio Emilia is recognised as just such a rich environment, where children are encouraged to invent with a wide variety of materials, often in unexpected ways, and where adults adopt an attitude of freedom towards children's work (Schwall, 2005). The impact of this model on embedded ways of thinking about early childhood environments has been noted (Fleer, 2003a).

The second aspect of the environment Laevers (2005) comments on is how well it supports children's processes of representation. It is the process of representing, Laevers suggests, that leads to the full experience of meaning, and recognising that "helps us to understand why the Reggio approach is so powerful" (p.24). In those centres art, in whatever media, is seen as a language for communication, and a way in which children can record their responses and in the process deepen their thinking (Gandini, 2005). The priority given to it is reflected in the provision of the atelier, the studio, and in the presence of the atelierista, who supports children in using the materials.

Laevers' (2005) insightful comments into the significance of the environment in relation to children's creative thinking suggest areas to consider in observing a centre environment; in particular his reference to the 'depth' of the environment, the potential for discovery, and the potential for children to re-present experiences and thinking. Would there be potential for discovery for children who had been at the centre for a long time? Art materials would be available, but would children be encouraged to use them in such purposeful ways? Or would some of these aspects be null curriculum? 
There is a range of other literature relating to the question of how effectively teachers support children's creative thinking. For example, children's dramatic play is understood to support their imaginative and creative thinking (e.g., Bodrova \& Leong, 2003), and yet scholars internationally have to argue for its continued inclusion in early childhood education (Paley, 2004) suggesting this may be a curriculum area under threat. However, this is not the case in New Zealand, where children's dramatic play is a near-universal feature of mainstream centre settings.

The teacher's role in supporting children's artwork is another area of tension that impinges on children's creativity. On the one hand there are recommendations to allow children to explore with encouragement but not intervention (e.g., Brownlee, 2004) and on the other, calls for teachers to demonstrate skills to children (e.g., Anderson, 2000). Familiarity with the philosophical approach of Reggio Emilia has fuelled the debate and led New Zealand teachers to increasingly question the accustomed practice of standing back from children's creative work (Farrant \& Wright, 2007). A final area of children's creative thinking that has received comment is children's humorous and playful exchanges. Alcock (2007), studying New Zealand children's imaginative verbal interchanges, found teachers rarely participated in such play, and so missed opportunities to extend it.

This résumé of points scholars had raised about children's creative thinking, increased my uncertainties about its positioning within a New Zealand early childhood centre.

\subsubsection{Thinking critically as curriculum?}

Critical thinking is the other aspect of young children's thinking that has been identified as marginalised (Robson, 2006). Again, both the physical environment and the pedagogical practices are seen as important. For example, Davis-Seaver (2000) describes changes she made to encourage children's critical thinking; she increased the range and accessibility of materials, added resources that would appeal to each of the senses, and removed labels from shelves so that children needed to sort out categories for themselves when tidying. Davis-Seaver also changed her teaching strategies. She elaborated her questioning when reading books to encourage children not only to predict, but also to identify grounds for their prediction. She allowed 
children to settle their own grievances, rather than imposing her decision, which encouraged them to consider other perspectives and evaluate alternative solutions. She talked with children about their thinking, followed up when she noticed confusions, and externalised her own thinking processes. Her account was useful in indicating the range of ways in which critical thinking might be promoted.

Three areas of potential curriculum that are relevant to critical thinking have drawn particular attention. These are: children as participants in democratic decision-making, children as resolvers of personal conflict issues, and children as philosophers and judges of moral issues. Scholars have argued for children's participation in decision-making as a way of understanding their democratic role (New et al., 2005; Nimmo, 2008) and as part of their cultural heritage (Pramling Samuelsson, 2000). A fundamental strand within the philosophy of Reggio Emilia is encouraging children to think and act for themselves, which is a reaction to the experience of Fascism and awareness of the dangers of obedience and conformity (Rinaldi 2006). Young Danish and Swedish children are introduced to democratic principles as part of their early childhood education; allowing children influence is seen as a human right and a prerequisite for effective learning, as well as meeting the requirement that teachers establish a democracy (Emilson, 2007). Examples include children having input into such decisions as which topic to study, what equipment to purchase, or on how a special occasion might be celebrated (Pramling Samuelsson, 2000). However, Emilson (2007) warns of "sham democracy" (p.35) when children are offered only limited alternatives. Calls for children's participation in democratic decision-making align with the literature on children's rights both internationally (Brandtzæg, 2006; Håøy, 2005) and in New Zealand (Dalli \& Te One, 2002; Te One, 2004, 2005). However, within New Zealand, only Te One (in preparation) has explored how children's rights are enacted within centre contexts, and no descriptions of New Zealand children taking a role in centre decision-making was located. This thesis will show that most centre decision-making lay outside the boundaries of the children's curriculum.

Children's role in resolving personal conflicts relates not only to their critical thinking but also to their ability to take other perspectives. Questioning the efficacy of teacher-led solutions has been a theme in recent research and professional literature. Reports suggest teachers tend to intervene with their own 
solutions rather than facilitating a child-focused resolution process (e.g., Rivalland, 2007), yet there is evidence that children's proficiency in resolving disputes increases when adults stand back (Corsaro, 2003; Singer, 2003). There is little evidence of the role that New Zealand teachers take, but Bernstone's (2007) finding, although not referring to conflict situations, that teachers rarely adopt the strategy of negotiation and allow the child equal power, suggests teachers' use of a facilitating role might also be rare.

Should children be introduced to philosophy? Among suggested potential benefits are that it "improves their ability to think carefully about what they and others claim to be right and true" (MacNaughton \& Williams, 2004, p.293), helps them to acknowledge multiple perspectives, and requires abstract thought. There are guidelines offered for facilitating philosophical discussions with young children (MacNaughton \& Williams, 2004). One particular aspect of philosophy-engaging children in assessments of moral rightness and wrongness-is also promoted (e.g., Knight, 2007), and there is supportive evidence for taking such an approach (Batycky, 2008; Knight, 2007; McCadden, 1998). No literature was located describing New Zealand teachers including philosophy or moral discussions as part of curriculum. Would this be an aspect of the null curriculum in this centre?

\subsubsection{Children's schematic thinking as curriculum?}

Scholars in both New Zealand (Harper, 2004; Meade \& Cubey, 2008) and the United Kingdom (Athey, 1999; Nutbrown, 2006b) advocate a focus on children's schematic thinking. Athey (1990) describes a schema as "a pattern of repeatable behaviour into which experiences are assimilated" (p.37). Having identified a child's schematic interest, such as rotation, teachers can then engage, sustain and extend that pattern of thinking by providing experiences and interactions (Athey, 1990; Nutbrown, 2006b). While literature around children's schema has been available for 20 years, in 2007 many New Zealand teachers were still unaware of schema although others were using the concept intensively (van Wijk \& Wilton Playcentre community, 2007). There has been little critical debate about schema, but it seems likely that the same criticisms levelled at a dispositional framework (in section 3.3.3 above) would also apply to schema. Reviewing this area of literature indicated it was uncertain whether children's schematic thinking would be considered as part of the curriculum. 
This review of literature describing how thinking may be privileged/marginalised within early childhood education has raised more questions than it has answered. Returning to the touchstone of the proposed list of habits of mind (Costa \& Kallick, 2000) shows there are many aspects of thinking that have not been covered. "Responding with wonderment and awe" (p.xiii) will be discussed in relation to children's spirituality in the next section, but what of the others? This thesis will suggest that while children's social learning was strongly promoted, aspects of children's critical thinking received less focus.

\subsection{What emotions might be marginalised or considered null curriculum?}

There is a small and disparate range of literature addressing questions of affect within early childhood education. While the original proposal from Flinders et al. (1986) included values and attitudes, along with emotions, as potential aspects of null curriculum, here the focus is on emotions as those other aspects are largely covered elsewhere within this chapter. This section opens with suggestions of how teachers' and children's expression of emotions may be supported/constrained within centre settings, and then considers three particular types of emotional experience within the early childhood curriculum: experiences of risk, and religious and spiritual experiences.

\subsubsection{Teachers' emotional expression as curriculum?}

Perhaps because it is widely accepted that teachers will be warm and responsive, references to the repertoire of emotions they display are rare. In the 1970s, King described English teachers as having "bright, smiling faces, eyes stretched open wide" (1978, p.71); he wrote of their "professional pleasantness", and their "affection" and "equanimity" in talking with children (pp.71-2). Brennan's (2005) description of New Zealand teachers suggests an equally positive but perhaps less studied persona. She reports there was "a culture of tenderness in this centre which was the outcome of teachers sensitively responding to children" (p.124). When children were distressed, teachers "created an affectiveintersubjectivity, mirroring then sharing the emotional state of the child" (p. 120). However, Brennan also notes "social and cultural restraints" prevented teachers from engaging in "emotionally charged affective displays" (p.118). Their "interactions tended to be positive and they did not engage in the full range of affective displays particularly in relation to expressing negative emotional states" 
(p. 204); when they needed to establish boundaries they often used strategies such as fun and humour to communicate important messages and soften demands.

Teachers' traditionally positive manner, that Brennan (2005) and King (1978) describe, has been critiqued. Scutt (1992) suggests it may not be "good for children to be reared, surrounded by female 'role models' who exemplify the perpetually unselfish, determinedly cheerful, self-abnegating icon" (p. 43) or for them "to grow up in a world where 'women's role' is seen as necessarily one of subordination" (p.43). More recently Woodrow and Fasoli (1998) identify "nurturing, empathy and an ethic of care" (p.41) as central values within early childhood, but again ask, (referring to Scutt) whether it is beneficial for children to be surrounded by such role models. The concept of the child as active learner is seen to reinforce the positioning of the teacher in this more passive and reactive role (Woodrow \& Fasoli, 1998).

Teachers' avoidance of disagreement in interpersonal professional relationships is a more frequently described example of their emotional constraint. Norberg (2006) describes teachers' reluctance to criticise peers, and identifies peace, harmony and consensus as core values. Nuttall (2004) found New Zealand teachers sidestepped misalignment in their perspectives on curriculum by refocusing discussion on day-to-day realities, thus avoiding conceptual debates. The daily requirement to work as a team meant maintaining harmonious relationships was seen as a priority. However, the fact that teachers in Reggio Emilia value "doubt, uncertainty and feelings of crisis" as "requirements for creating new thinking and perspectives" (Dahlberg \& Moss, 2005, p.18) indicates a cultural dimension to teachers' emotional expression. Questions about teachers' emotions are not often raised, but these comments collectively suggest expression of some emotions by teachers might be null curriculum.

\subsubsection{Children's emotional expression as curriculum?}

There are also intriguing suggestions about what aspects of children's emotional expression might be defined as inappropriate. In a more fundamental way than teachers, children are also seen to learn to moderate their emotional reactions in centre settings and to act in 'socially appropriate ways' (Bergen, Reid \& Torelli, 2001).

When children enter daycare as infants and toddlers, "all leaky bodies and unregulated desire, fluids pouring out of orifices insufficiently closed to the world," they are subjected to multiple methods designed to teach them 
control, to move them from "unbridled expressions of bodily pleasure to socially sanctioned forms of play, from excessive pleasure to good clean fun” (Tobin, 1997, p.19). (Cannella \& Viruru, 2002, p. 206)

The same notion is reflected, in more measured words, in the goal in Te whäriki for children to "gain confidence in and control of their bodies" (Ministry of Education, 1996, p.86).

A part of learning to moderate their emotions is the need for children to learn the particular norms of the setting (Apple \& King, 1977; McCadden, 1998). The importance teachers assign to harmonious group relationships, and the impact this has on children, has received particular comment. The commonly made comparison is between collectivist cultures which tend to prioritise harmonious group relationships and cultures with an individualistic focus which are more likely to encourage the expression of feelings (e.g., Gonzalez-Mena, 1997) but other research suggests maintaining harmony within the group may also be a deep-seated value within early childhood education. In recent Australian research (Rivalland, 2007) teachers were found to attribute "more importance to maintenance of group cohesion than to care and education" (pp.33-34). "I observed that this community belief led to constant redirection of children and indicated tension between the deep core beliefs and other articulated beliefs of childcare professionals, such as freedom of choice and respect for children's rights" (Rivalland, 2007, p.34). Would New Zealand research identify a similar commitment to group cohesion? There was little to indicate this beyond Brennan's (2005) description of teachers using teacher-led rituals to develop a strong sense of community.

One further aspect of children's emotional expression is the disapproval of physicality and noisiness. 'Quiet voices' is a consistently reported rule (Apple \& King, 1977; King, 1978; McCadden, 1998) and teachers are seen to respond more positively to children who play quietly (Fleer \& Robbins, 2007). Yet there are arguments in favour of children's rough physical play. Hughes (2003) cites research linking rough-and-tumble play to frontal lobe development, and suggesting stifling it may be related to the development of Attention-deficit Hyperactivity Disorder (ADHD). Jarvis (2007) suggests such play has value because it "puts children into real situations where they can practice spontaneous and autonomous competitive and co-operative interactions simultaneously, developing complex social skills that 
fundamentally underpin primate adult life" (p.185). (Teacher attitudes to superhero play are considered below in section 3.5.6.)

Teachers' focus on group cohesion and avoidance of dissension may also underpin their typical reaction to physical conflict, which is to eliminate it as quickly as possible (Danby, 1997). Gonzalez-Mena (1997) recognises "Keeping one child from hurting another, even slightly, is an important value for the typical white, European-American, early childhood practitioner" (p.86). Cultural differences in teachers' reaction to physical conflict can affect how children express themselves; for example Japanese teachers consciously provide children with fewer resources during the year so that children have the chance to learn to handle conflicts (Gonzalez- Mena, 1997). Jordan, Cowan and Roberts (1995) suggest that prohibiting physical violence forces children to develop other ways of expressing emotion in conflict situations.

Although there is little that is definitive in this array of thoughts about children's and teachers' emotional expression, they indicated these might be interesting aspects of curriculum to consider further, particularly because no recent New Zealand research had addressed these questions. The final three topics are more obvious areas of curriculum debate.

\subsubsection{Experience of risk as curriculum?}

Keeping children safe from physical harm is a central tenet of early childhood practice, arising from the positioning of children as vulnerable (Nimmo, 2008). However, it leads adults to limit children's experiences, and in particular their outdoor play, and recent writers ask what children may be missing through lacking opportunities to experience and overcome a feeling of potential risk (Maynard, 2007; Stephenson, 2003; Tovey, 2008). Descriptions of Scandinavian forest kindergartens where teachers value sites more highly for the potential excitement and challenge they offer than for being hazard-free (Lysklett, 2006) provide an alternative vision that is influencing teachers in other countries (Maynard, 2007). Would experiences of physical risk be null curriculum in this setting? Or would this be an aspect of curriculum contested by children and teachers? 


\subsubsection{Religious and spiritual experiences as curriculum?}

The place of spiritual and religious experiences in curriculum has received little comment until recently, which is perhaps surprising given the traditional emphasis on holistic approaches within early childhood education (Ryan \& Stower, 1998). Within New Zealand spirituality has particular prominence because the Te whäriki aspiration statement includes the words "to grow up... healthy in mind, body and spirit" (Ministry of Education, 1996, p.9). In contrast, there is less likely to be New Zealand literature about religious experiences within early childhood education, given the legal commitment to secular public education. In fact, one notable public debate was about potential encroachment of religion into curriculum, when a state-funded kindergarten gave children Easter buns with crosses marked on them.

Recent writers have argued for the inclusion of both spirituality and religion within early childhood curriculum, stressing both can be part of day-to-day life. The New Zealanders, Bone, Cullen and Loveridge (2007), advocating for a spiritual dimension in early childhood curriculum, introduce the idea of "everyday spirituality" (p.344), an appreciation of the potential spirituality in everyday events and interactions. While acknowledging that for some including spirituality may remain controversial, they detail the ways in which it is embedded within the concepts, both Māori and English, of Te whäriki. In a practical vein, the American scholar Greenman (1998) offers suggestions for providing experiences of awe and wonder.

Even less reference is made to young children's religious experiences in the general early childhood literature, although there is more within religiously oriented journals. Ryan and Stower (1998), drawing on Robinson's (1977) exploration of adults' memories of childhood religious experiences, describe them as "mostly part of ordinary, everyday living and irrespective of contact with any particular religious tradition or group" (p.3). They suggest teachers help children find the language to express their experiences and provide environments and personal care that "respect, encourage and protect" (p.3) these. The paucity of literature in this area, and the fact that it is focused on including religious and spiritual experiences, suggests these may be areas of null curriculum. 
This completes the review of literature addressing the question of what constitutes null curriculum in early childhood education. In the following section of this review, attention turns to writing on children's peer groups and peer culture, and in particular to the question of how children themselves may influence and enact the boundaries of curriculum.

\subsection{What is the role of the peer group in influencing and enacting curriculum boundaries?}

The final section addresses the question of how might children influence and enact curriculum boundaries, by considering the literature on peer groups and peer culture. This means a shift to more certain territory; to return to the opening metaphor of the pulled rug, here there are far fewer loose ends because this is an area that has attracted more research and writing. Therefore only those aspects of literature that proved to be relevant in this thesis are reviewed. These are children's collective enjoyment of each other, their resistance to adults, the exercising of power amongst themselves, and their shared interests (with a particular focus on gender). Given the dearth of research on children's peer groups within New Zealand (Alcock, 2005), these are all areas where it is anticipated this thesis may offer insights that are relevant within New Zealand, and perhaps also internationally.

Two writers have been influential in highlighting the wealth of curriculum derived from, and shared among, young children. Corsaro's seminal works (1985, 1997, 2003; Corsaro \& Nelson, 2003) explore peer culture in American and Italian settings and Paley (e.g., 1984, 1986, 1995, 2001, 2004) draws unparalleled attention to young children's peer culture in North American classrooms. Although Corsaro (1985) indicates the notion of a shared childhood culture is "conceptually problematic" (p. 172) because most elements of peer culture originate from children's reactions to or against aspects of the adult world rather than being passed child to child, it is nevertheless pertinent to review what aspects of peer culture have been identified in other settings. Corsaro $(1997,2003)$ identifies two central themes in children's peer culture. These are: "Children make persistent attempts to gain control of their lives and ... they always attempt to share that control with each other" (1997, p.118, italics in original). These are similar to the two elements of relational morality identified by McCadden (1998): the desire to make connections with others and to be accepted, and the need to be able to access people and resources. The significance of connecting with others is reiterated in Paley's (1986) theme of friendship, which 
along with fairness and fantasy are the elements she sees as dominating children's thinking. Kyratzis (2004), in a review of wider research on peer cultures, lists a range of more specific themes including constructing a gender identity, resisting adult culture and issues of inclusion and power in the peer group. The analysis within this thesis offers a somewhat different interpretation of the central themes of children's peer culture.

\subsubsection{Enjoyment of doing things together}

The pleasure children gain from being in each other's company is a common theme; Corsaro (1997) writes "children immensely enjoy simply doing things together" (p.140) and considers social participation an "overriding" (p.119) concern. Singer (2003) believes young children are focused on their social world, are inherently motivated to form and maintain social relationships, and that "children's interest in one another and the value of these contacts are often underestimated" (Singer, 1996, p.36). New Zealand studies also underline children's interest in being part of the peer group. 'Being a friend' was one of six discourses used by 4-year-old children in kindergarten (Carr, 1997a, 1997b); children's 'longing to belong' emerges as a theme in Brennan's (2005) study; Alcock (2007) identifies 'peer togetherness' as being an object for most children.

However, the challenge for children in learning to get along with others is also recognised. Making friends, gaining access to groups, and maintaining interaction are demanding tasks (Corsaro, 2003; Singer, 1996). Corsaro (2003) suggests "the social ecology of most preschools increases the fragility of peer interaction. A preschool play area is a multiparty setting much like a cocktail party with lots of clusters of kids playing together" (p.40). But getting on with others is valued as a "major step in children's acquisition of social knowledge" (Corsaro, 1985, p.121), and enables children to learn skills like cooperating and sharing, empathy, perspective-taking and conversing (Wiltz \& Klein, 2001). (Literature on the role of teachers in supporting children's peer interactions was referred to in section 3.2.5 above.)

Children use a range of strategies to communicate, engage others and form alliances, and there is particular detail on their verbal alliance-building strategies. Kyratzis (2004) lists repetition, singsong intonation in jokes, songs, and sound play as examples. Corsaro (1985) includes children's sharing of jokes and riddles, and gives accounts of group glee, which involved laughter, screams, giggles and jumping up and down and often occurred during teacher-directed activities. 
Scatological references are used by children to simultaneously establish a sense of group and resist adult norms (Alcock, 2007; Kyratzis, 2004), although Corsaro (1985) found these could also be used to tease, insult or threaten peers. Alcock (2007) describes New Zealand children's playful chanting, and draws attention to a wider range of strategies, verbal and non-verbal, which they use to engage each other and communicate.

\subsubsection{Resistance to adults}

Resisting adult cultures and values has been seen as mechanism for strengthening group identity (Alcock, 2005, 2007; Brennan, 2005; Corsaro, 2003; Kyratzis, 2004), and is used by children as young as two (Rutanen, 2006). Cobb, Danby and Farrell (2005) suggest such resistance is children's reaction to increasing levels of adult-imposed governance and that play provides "a vehicle through which they can experience the adult power they observe around them" (p.137).

In particular, children's resistance to adults' rules is widely reported and such subversion is seen to contribute to shared group identity (Corsaro, 1985, 1997, 2003; Jordan et al., 1995; Kyratizis, 2004). McCadden (1998) found every child resisted in some way, but boys and those who were most culturally different from the teacher demonstrated the greatest resistance. Corsaro $(1985,2003)$ uses Goffman's notion of secondary adjustments in describing children's reaction to rules. Secondary adjustments "involve using legitimate resources in devious ways to get around rules and achieve personal or private needs or wants" (Corsaro, 1997, p.133). The rules children most energetically evaded were rules regarding play areas and materials, such as the behaviours for indoor and outdoor play, rules defining use of resources, and against guns and shooting, and rules around cleaning up. Children also resisted the prohibition against bringing things from home, and Corsaro (2003) recognises the subsequent covert sharing of possessions as important peer episodes. Brennan (2005) found New Zealand children also use a range of secondary adjustments. Along with openly challenging rules and practices, they adapted rules to their own purposes, followed their own rules rather than the official one, and distracted the gatekeepers or made use of others to avoid participation. Using a sociocultural lens she identifies such resistance as a way in which novices challenge more experienced cultural members to review and readjust practices and norms, a process which allows the culture to evolve. Alcock (2007) highlights the playful 
quality in episodes of New Zealand children's playing around with and challenging adult rules. Waksler's (1991) reminder of the embedded assumption that adult rules will always be right, and children's reasons for rule-breaking will always tend to be wrong, was a valuable caution. Because children's resistance was found to be a way in which they challenged teachers' definitions of curriculum boundaries, this thesis will contribute further to the insights from other recent New Zealand research (Alcock, 2005; Brennan, 2005).

\subsubsection{Children's use of power with peers}

Alongside resistance to adult-imposed rules and requirements, the wider issue of children's use of power amongst themselves is relevant given the research focus on teaching and learning amongst children.

Exercising of power is inherent in protection of their interactive space (Corsaro, 1997, 2003) and is an issue with which Paley (1992), as a teacher, tussled. A range of power-oriented strategies used amongst children is described in the literature. For example, they may express themselves vocally by threatening violence, name-calling, blaming, and issuing threats, using degrading or hurtful language, loud and domineering voices, and also by using non-verbal vocalisations such as shouts, grunts and screams (Gruss, Jackson, Grimson \& Hedgcock, 1998). They may invoke adult-derived rules in order to impose their will on peers (Cobb et al., 2005; Jordan et al., 1995). They may use poweroriented strategies to include or exclude others (Dockett \& Fleer, 1999), which can lead to children creating inclusive/exclusive membership categories (Cobb et al., 2005) or taking up discourses that reproduce inequitable social relationships (Wohlwend, 2007). It is these aspects that lead MacNaughton (2003b) to label play as "dangerous":

... through play children can construct play worlds in which they practice [sic] and learn to be unfair, to compete for power and to fear the social diversity in their daily world. They can create and experience racism, sexism, homophobia and classism ....They practice what they learn from the adult world and each other about gender, sexuality, violence, love, hate, power, friendship, exclusion and inclusion. Because of this play can be a dangerous place to be for many children. (MacNaughton, 2003, p.58)

Insights such as this led the New Zealander Ritchie (2001) to comment that adults need to intervene in oppressive play rather than "allowing their uncritiqued pedagogic discourses to render them powerless" (Ritchie, 2001, p.144). (Children's use of power within their gendered roles is discussed in section 3.5.5 below.) 
Exercising power-oriented strategies with peers can lead to conflict, which Corsaro (2003) sees as "a central feature of kids' peer culture" (p.193). Some researchers report conflict rarely escalates into physical violence. For example, an Australian study found that although children were very physical, violence was almost absent and was ruthlessly sanctioned which led children to learn to use other strategies (Jordan et al., 1995). However, other Australian researchers give a different picture. In a survey of preschools (Gruss et al., 1998), in almost every setting children were said to react to others in a physical way, with a 7:1 ratio of male to female. Other research confirms a higher level of aggression among boys (Jordan, 2002; Sims, 2000), and Hadley and Nenga (2004) report when girls did fight, it was in the context of a television-derived script. The topics of children's use of power, both physical and social, against their peers were areas where there was no recent New Zealand research literature, and so was a topic where this thesis might offer insight.

This section has suggested some strategies children may use in enacting boundaries of curriculum; the following section considers how children may influence the scope of curriculum by considering the interests young children share with their peers.

\subsubsection{Children's shared interests}

A number of interests have been linked to young children's peer cultures.

Children's interest in popular culture, described above as a contested curriculum topic, is seen as drawing children together, particularly in light of teachers' relative ignorance (Hadley \& Nenga, 2004). A commitment to participation and friendship with peers (discussed in 3.5.1) and an interest in gender have both been seen as key interests for young children. But before addressing these in detail, there are a number of other more specific interests that have been described.

A concern with size is recognised (Corsaro, 1997, 2003; McCadden, 1998); "being a bigger kid is better than being a smaller kid, since bigger kids can do more adult-like things" (McCadden, 1998, p.75). Carr (1997a) notes the significance of "being big" and "being nearly five" (p.228) among New Zealand children. Corsaro links the concern with size to children's predilection for high places and Paley (1986) links it to their fascination with birthdays and with being older. An interest in teachers' personal lives was seen by Brennan (2005) who 
also found children used other children's parents as a source of information about other families. A concern for the physical welfare of others has been noted (Brennan, 2005; Corsaro, 1985); Brennan describes children's almost obsessive interest when an ambulance was called for an injured teacher. Finally, dramatic play has been recognised as a central aspect of young children's culture (Corsaro, 2003; Paley, 2004). Corsaro (2003) sees such play provides children with a context in which they can explore shared concerns, and deal with issues such as danger and death. Power is a recurrent motif in children's dramatic play (Corsaro, 2003; Dockett \& Fleer, 1999), and Lofdahl (2002) suggests the way children handle power in play offers insight into their understanding of "how to deal with authorities, and how to gain authority" (p.45). (The particular case of superhero play is discussed in the following section.)

\subsubsection{Gender}

Kindergarten is a triumph of sexual self-stereotyping. No amount of adult subterfuge or propaganda deflects the five-year-old's passion for segregation by sex. (Paley, 1984, p. ix)

Young children's growing awareness of gender differences and the construction of their own gender identity is a central theme of peer culture (Kyratzis, 2004), particularly for older children in a centre (Corsaro, 1997, 2003; Fleer, 1998), although children as young as three show gendered patterns in their play (Fabes, Martin, \& Hanish, 2004). Six months of observations in a kindergarten led Blaise (2005) to recognise "the hard and important work [children] ...do constructing gender" (p.114). It is also recognised that children take up these roles "in ways not necessarily compatible with the ways teachers and parents ... [are] telling them gender should be done" (Davies, 1993, p.xvii), and adult attempts to introduce equity considerations may only "affect the minor detail of this difference, and then only if that detail ... [has] not become a key signifier of masculinity or femininity" (p.xvii).

The significance of clothes, possessions, cultural knowledge and physical movement in gender discourses is recognised (Giugni, 2006; Hadley \& Nenga, 2004). Popular culture products are central (Giugni, 2006; Hadley \& Nenga, 2004), and are "key signifiers of power, knowledge and authenticity" (Giugni, 2006, p.212). Children use products to create their own performance scripts (Giugni, 2006), to display knowledge, as a resource to draw on in play (Hadley \& Nenga, 2004) and as grounds for including or excluding others (Giugni, 2006). While over 
the years the referenced products have changed-Darth Vader, Batman and Star Wars (Paley, 1984), Spice Girls, Batman and Barbie (Brennan, 2002), Batman and Barbie (Giugni, 2006) — their significance appears to remain unabated.

Girls' gendered discourses identified by Blaise (2005) were: wearing femininity, body movements, makeup, beauty, and fashion talk but she found physical presentation was the most obvious way in which girls practised gender. Some adopted the model of "girly girls" (p.107) and wore clothes that were pink, ruffled, cute. Others were the "cool girls" (p.107) and aimed to be sophisticated in their dress. Girls made use of dress-up clothes to transform themselves into beautiful princesses. In a similar way, boys have been seen to construct identity through wearing superhero insignia (Giugni, 2006), through their style of play (Blaise, 2005) and by dissociating themselves from girls' gendered knowledge (Brennan, 2002).

Given the energy children invest in defining gender differences, it is unsurprising that differences between boys' and girls' groups have been noted. In girls' groups dominance hierarchies tend to be more fluid, cooperation and verbal interaction are often emphasised, girls tend to promote group harmony, and are more likely to support adult-structured activities that are governed by strict social rules (Fabes, Martin, \& Hanish, 2004). Girls are more concerned to appear caring (Jarvis, 2007). Their dramatic play is about relationships between characters, their scenes last longer, and there is more communication between characters and more shared activities (Kristensen, 2006). MacNaughton (2000) records girls typically play in areas away from the boys, in "decorative, gentle, passive and domestic areas" (p.112). Watson (2005) comments little concern has been expressed about the typically passive, quiet teacher-pleasing quality of girls' play, which perhaps reflects Paley's (1984) conclusion that she has drawn her cues from the girls, and her "curriculum has suited girls better than boys" (p.105).

Fabes et al. (2004), summarising research, report boys' groups tend be larger and have a well-established hierarchy, boys play in more public places, their play is rougher and more often involves physical contact, fighting and taunting. Boys' play often includes the roles of antagonist and protagonist, and has a quality of instability which contributes to the conflict and tension (Kristensen, 2006). Boys tend to be more concerned with portraying toughness (Jarvis, 2007). 
Older boys and girls are seen to play less frequently together (Corsaro, 1997; Hyun \& Dong, 2004), and when they do play together their activities often take the form of borderwork, activities which heighten awareness of gender differences, and so strengthen the demarcation (Corsaro, 1997). It is usually girls who instigate mixed gender games (Corsaro, 1997; Jarvis, 2007) and boys tend to respond with feigned threatening behaviour (Jarvis, 2007).

\subsubsection{Gender-power issues}

There is a range of literature describing the complex interweaving of power and gender issues (e.g., Dockett \& Fleer, 1999; MacNaughton, 2000), and boys' use of power has received particular focus. Davies (1989) describes boys' power being demonstrated in their "domination in public spaces, particularly of females but also of smaller boys" (p.91), and notes the importance of gun play in this process. Groups of boys, sometimes earning the label of 'the bad boys', have been described in the literature:

There was a gang of macho boys at each of the places I studied. These were the boys who had, as far as one could see, successfully achieved the hegemonic form of masculinity.... Generally they roved in packs, were dedicated users of guns and were often aggressive towards girls and towards younger or weaker boys. They were undoubtedly the lords or superheroes of the playground, and always made their presence felt. (Davies, 1989, p.122)

Jordan (2002) uses the label "fighting boys" (p.32) to describe the boys who resist, who hit out frequently and get punished frequently. Their presence creates difficulty for other boys who experience an inevitable tension in relation to their masculinity (Davies, 1989; Jordan, 2002).

The most frequently described boys' play episodes are the fast-paced, chasing and shooting play based on media-derived themes, which are labelled superhero play. In such play boys are seen not only to be defining themselves in relation to the girls (Jones, 2002; Paley, 1981), but also in opposition to teachers (Watson, 2005). At times teachers feel intimidated by such play (Browne, 2004). Scholars highlight the far-reaching implications of how children position themselves in relation to gender (Davies, 1989; Grieshaber, 2001; MacNaughton, 2000). Keddie (2003) points out: "the social dynamics of peer culture are potent in constructing and regulating restrictive understandings of masculinity mobilised around aggression, violence and physical domination and underpinned by the constitution of females and femininity as the negative 'other'” (p.300) and that research tells 
us “young children's understandings and behaviours are far from innocent, harmless, natural or inevitable" (p.301). These thoughts resonate with MacNaughton's (2003b) comment quoted above that play is "dangerous". Superhero play is seen to highlight the conflict boys face between the teacherpromoted value of non-violence and media-derived images of masculinity linked to violence, power and autonomy, which requires them to confront issues of gender identity much earlier than girls (Jordan, 2002).

How teachers should respond to boys' superhero play is a point of debate (Carlsson-Paige \& Levin, 1990; Watson, 2005), with many teachers ambivalent in their reactions (Browne, 2004) or even banning it (Jordan et al., 1995). Rejection of superhero play is usually not linked to its origins in the mass media, but rather to the violence it can promote (Watson, 2005). Browne (2004) suggests teachers' ambivalence may also reflect their realisation that such play "is essentially a display of hegemonic masculinity" (p.92). However, there are also arguments offered for working with children's superhero play. For example, it can be a route to exploring media images more thoughtfully (Carlsson-Paige \& Levin, 1990); and not supporting it may damage a child's self esteem (Watson, 2005). While only Watson's work refers to New Zealand contexts, the collective evidence suggests superhero play would be a feature of the boys' play and that teachers might be ambivalent about it. Discussion in Chapter 6 will show that teachers in the centre did indeed share this ambivalence.

While boys' exercise of power is more frequently discussed, girls' use of power has also received comment. Firstly, the point is made that through boys' experiments with exercising power, girls (and female teachers) are experiencing being dominated (Browne, 2004). When girls do instigate interactions with boys' groups (Corsaro, 2003; Jarvis, 2007), the resulting run-and-chase play (Corsaro, 2003) can be seen as an expression of this dominant-submissive relationship. However, there are ways in which girls themselves exercise power. When boys move into an area, girls will tend to retreat to a space they can dominate (MacNaughton, 2000), and they tend to exercise their control of an area through language rather than physical aggression (MacNaughton, 2000). Like boys, they use cultural products to signify knowledge, authority, and power amongst themselves, with peers included and excluded based on possession and knowledge of such products (Giugni, 2006). There was little recent New Zealand evidence, 
beyond a passing comment by Watson (2005), to suggest how girls might exercise power among their peers. This will be an area where the findings from this thesis provide evidence.

It is important to acknowledge the issues children who choose not to adhere to gender demarcations promoted by their peers may face. Aligning oneself with the gendered definitions of what it is to be girl or boy is recognised as "high-status" (Davies 1989, p.126) and an indicator of popularity. However, some children refuse to adhere to the promoted gender roles and behave in ways that compromise their own, and their peers' understanding of what it means to be a boy or a girl (Davies, 1989; MacNaughton, 2004). Davies reports that for some children, their assigned gender role is "a straitjacket they have a lot of trouble wearing" (p.128). Other children will often act collectively to reinforce gender when an individual deviates (Dockett \& Fleer, 1999).

Interestingly, given the depth of research on gender-related issues discussed above, very little recent New Zealand research has taken a gender focus (Norris, 2001). This made it likely that gender-related issues would be an area where this thesis might contribute useful new perspectives.

This section completes the review of literature suggesting ways in which children may influence or enact the boundaries of curriculum in a centre. As the researcher, I was left with a long list of leads to consider once observing began. Although relatively few writers had considered the impact centre architecture and design might have on children's curriculum experience, there were tantalizing leads to follow particularly relating to the site, the interior layout and the provision of withdrawal spaces. Writing on resources suggested these warranted further scrutiny in terms of the range, accessibility and teacher control. In reviewing others' suggestions for what aspects of curriculum might be marginalised or considered null curriculum, the framework of content, intellectual processes and affect was used. A range of content areas had been identified-'real' tasks, use of ICT, the body, sexuality, death, disaster, issues of social equity and social justice - and it was noted that generational assumptions and a positioning of children as less competent underpinned many of these. Children's shared interests in friendship and popular culture had been offered as ways in which they might enlarge the scope of curriculum. Reviewing literature that touched on what might 
be marginalised ways of thinking and learning in early childhood education indicated two areas for particular focus: firstly, being sensitive to the impact of culturally privileged ways of learning/teaching and, secondly, exploring in detail the kinds of thinking and learning that were encouraged and supported in the setting. In the relatively small pool of writing considering how emotional dimensions might be marginalised, there were descriptions of both teachers and children avoiding expressions of some emotions. Other possible areas of marginalised curriculum were religious and spiritual experiences, and risk-taking. The final section of the review considered the role of the peer group in influencing and enacting curriculum boundaries. Four areas of interest were identified: children's enjoyment of doing things together, their resistance to adults, the exercising of power amongst themselves, and their shared interests, particularly gender.

Just as I, the researcher, was left with a long list of possible leads, so you as reader, are left with an array of potential ways in which this thesis may contribute to understandings of curriculum, and the process by which curriculum boundaries are established. The answers will not come in the next chapter-there the methodology used in the thesis is outlined. However, in the following four chapters, where the findings from the thesis are presented, many of the questions raised in this chapter will be resolved. 


\section{CHAPTER 4}

\section{METHODOLOGY}

By giving children a voice and listening to them, we may counterbalance our adult tendency for routines, taken-for-granted knowledge and orthodoxy. (Singer, 2005, p. 618)

This chapter describes the planned methodology and explains how those plans transpired. The first section introduces the generic inductive qualitative model (Hood, 2007) which guided data generation and analysis and the second outlines the planned methodology. The human dimension is covered in the third sectionthe practical and ethical processes of negotiating access to a centre, gaining ethical consent/assent from participants, and the reality of living those ethical commitments. The fourth section describes changes that occurred in implementing the methodology, and introduces the research strategies used with children. The final section outlines the process of analysis, both during and after the time in the centre.

\subsection{The route from constructivist grounded theory to the generic inductive qualitative model}

I had used principles of grounded theory to guide the process of data generation and analysis in earlier research (Stephenson, 1998), and planned to do the same here. Revisiting the literature (Bryant \& Charmaz, 2007) confirmed this approach was suited to the broad, open-ended scope of the topic. The intertwining of analysis and data collection, with each informing the other, had proved fruitful in the past, although I had lingering doubts about whether the final output would be theory. Constructivist grounded theory (Charmaz, 2003, 2006), in particular, seemed the appropriate model to use. Unlike the objectivist approach, constructivist grounded theory acknowledges the social context, the researcher's influence, and the interaction between researcher and participants (Charmaz, 2006). Methods play a less central role and the aim is "to show the complexities of particular worlds, views, and actions" (Charmaz, 2006, p.132). Knowing resultant conclusions were considered "plausible accounts" rather than "verified knowledge" (Charmaz, 2006, p. 132) was also reassuring. 
However, Hood's (2007) incisive discussion of the parallels and differences between grounded theory (GT) and the generic inductive qualitative method (GIQM) forced me to acknowledge that, like "most other researchers claiming to be doing GT" (p.164), I was not meeting the criteria. In outlining what constitutes the generic qualitative model, Hood refers to Maxwell's (2005) description of the key aspects. Hood's summary is quoted in full because it provides the context for the following discussion of data collection and analysis:

(1) Questions get at processes rather than 'variance.' Process questions ask 'how' rather than 'whether or not,' or 'how much' (Maxwell, 2005: 74-75).

(2) Researchers normally move back and forth among data collection, data, analysis, study design and focus, and emerging theory. However the GIQM allows for the use of existing theory in developing one's question as well as in interpreting results (Maxwell, 2005: 43-47).

(3) Samples are purposeful ones that allow theoretical (cross population) generalizations (Maxwell, 2005: 115-116).

(4) Analysis of data begins with the first observation and continues as additional data are collected.

(5) Researchers write copious memos of many sorts (concept maps, interpretive memos, research process memos) from the start of the project.

(6) Coding focuses on themes and sometimes theoretical categories.

(7) Data collection stops when additional cases no longer add new information. (Hood, 2007, p.153)

Hood (2007) identifies the essentials of grounded theory as "theoretical sampling", "constant comparison of data to theoretical categories", and "focus on the development of theory via theoretical saturation of categories rather than substantive verifiable findings" (p.163). While these may be implemented in varying ways, Hood argues they are essential elements, that the fundamental processes of grounded theory have been misunderstood, and that the distinction between grounded theory and the generic inductive model has been blurred.

Reading Hood (2007), and other definitions of grounded theory (Bryant \& Charmaz, 2007), convinced me these prerequisites were not met in this thesis. The criteria for theoretical sampling were not met because the selection of six focus children towards the end of the data generation was partly guided by demographic detail (including boys/girls, younger/older). The requirements of constant comparative analysis were not met because the focus was on emerging themes rather than theoretical categories. Finally, the analysis did not produce theory, but rather met the goal of the generic inductive model for "interpretation of rich data" 
(Hood, 2007, p.156). While these were disquieting conclusions, Hood (2007) affirms the potential of the generic inductive qualitative approach. The following sections of the chapter outline the planned methodology, the reality of implementing it, and describe how the generic inductive approach underpinned this. The next section introduces the ethnographic approach which provided the central data-generation strategy, outlines the plans for child-focused research strategies, and explains the selection of six focus children towards the end of data collection.

\subsection{Planning the research}

\subsubsection{Using ethnographic methods}

Decisions about methodology were intertwined with the initial refinement of the topic, and occurred in two stages. The choice of an ethnographic approach came first, and subsequently it was decided to combine this with strategies foregrounding children's perspectives. Several factors influenced the decision to use ethnographic methods. First, this approach 'fitted the context'; the research strategies of observing, writing notes and taking photographs are familiar teacher behaviours and therefore less likely to disrupt centre life. I already had experience of using ethnographic methods (Stephenson, 1998), and they had also been used effectively by others investigating curriculum issues in early childhood and early school settings (e.g., Brennan, 2005; McCadden, 1998; Millei, 2005; Skinner et al., 1998) and in studies of young children's peer culture (Corsaro, 1985, 2003; Corsaro \& Molinari, 2000; Hadley \& Nenga, 2004; Taylor \& Richardson, 2005). Within early childhood education there are calls for more ethnographic research which "can paint in the fine-grained reality" of children's centre lives (SirajBlatchford \& Siraj-Blatchford, 2001, p.194).

While there is continuing debate over the categories and parameters of ethnography (Denzin \& Lincoln, 2002; Jenks, 2000), definitions are typically broad and accommodating. For example, Hammersley and Atkinson (1995) interpret ethnography “in a liberal way" (p.1) as:

the ethnographer participating, overtly or covertly, in people's daily lives for an extended period of time, watching what happens, listening to what is said, asking questions - in fact, collecting whatever data are available to throw light on the issues that are the focus of the research. (p.1) 
In adopting any research approach, it pays to be aware of the critiques, in order to avoid the pitfalls. Critiques of ethnography fall into three categories: the domination of the researcher, the robustness and rigour of the research (validity and reliability), and the claim to generalisability. The steps taken to address these are outlined below.

There are two aspects to potential researcher domination. The narrower aspect refers to researchers failing to address issues of researcher bias. Denzin's (1997) description of ethnography as "that form of inquiry and writing that produces descriptions and accounts about the ways of life of the writer and those written about" (p.xi) is a reminder of how strongly the researcher lens can colour a project. Given that, at heart, ethnography depends on the interpretation of the researcher, and that "detachment and author objectivity are barriers to quality, not insurance of having achieved it" (Lincoln, 2002, p.334), the following steps were taken to keep my lens visible. The first was the description in Chapter 1 of the mix of factors that led me to the topic. Before beginning fieldwork, following Carspecken (1996), I also undertook the task of interrogating myself to identify assumptions and prejudices and shared this document with colleagues, whose comments deepened my awareness of habitual positionings. An ongoing journal provided a place for reflecting on the complexities in my researcher role. During the analysis, records were kept of coding decisions as a trail of evidence showing the categorisations and inferences, and the logic of the steps taken. Lastly, the three strategies of dislocation, introduced in Chapter 2, were adopted as a way of disrupting habitual patterns of thinking.

The broader aspect of researcher dominance relates to issues of power and colonialism (Cannella \& Viruru, 2004). It is the researcher who "maintains the power to decide who and what to study and how to represent their voices" and takes the role of Expert gathering information from the Other (Kincheloe \& McLaren, 2005). The apparently benevolent decision to foreground children's perspectives also meant I was positioning children as Other and therefore implying the centrality of the adult (Alldred, 1998). Children did not choose the topic, devise the methods, or consider what the data might mean (MacNaughton, Smith \& Davis, 2007). How can this critique be answered? 
Addressing the power imbalance between researcher and children is a central feature of the methodology. Foregrounding children's perspectives was intended to shift the power balance in their direction. Developing other research strategies in the context was an attempt to find ways that would enable these children to share their thoughts. The commitment was to respectful interaction, empathetic listening and engagement, and although the commitment to foreground children's perspectives was most significant during data generation it acted as a touchstone at all stages. Finally, it might be argued that the critical pedagogical framework for this thesis aligns it with Cannella and Viruru's (2004) proposals for decolonialist research. They ask, "How does one co-construct a new kind of research with children that reflects their perspectives?" (p.150). This thesis, in a small way, attempts to move in that direction.

The second category of criticisms of ethnography concerns its claims to validity and reliability, and its robustness and rigour as a research approach. There are suggestions for strengthening validity and reliability (Carspecken, 1996) and proposals to use alternative criteria (Pole \& Morrison, 2003). Pole and Morrison provide a useful amalgam of strategies suggested in the literature:

1. Checking with informants, but also adopting a critical attitude towards what informants say (p.102),

2. Seeking alternative explanations (p.103),

3. Checking ethnographer effects (p. 103),

4. Representing the range of voices in the field (p.103).

Each of these was drawn on to increase the rigour of this thesis.

Strategies to enhance the rigour of fieldwork included the use of multiple recording devices (note-taking, tape-recording, photography) as part of observing (Carspecken, 1996); "prolonged engagement" (p.88) in the field; using lowinference language in fieldnotes and recording speech verbatim whenever possible; and recording actions and expressions factually (Carspecken, 1996). Children had multiple opportunities to respond which allowed for further triangulation. Artefacts in the centre-documents, photographs, resourcesformed part of the data set which allowed for elements of triangulation of data used in the analysis. Parent interviews and the informal comments of teachers provided further triangulation through the fieldwork. 
Once coding of data began, some of the initial categories used were manifest (Berg, 2004); for example, all data relating to a child were coded to that child. Other initial categories were broadly analytical (Berg, 2004); for example, some data were coded "null curriculum" reflecting the focus of one research question. At this stage in particular, thoughts about possible future lines of analysis were recorded as memos (Hood, 2007). In identifying potential patterns and themes (Graue \& Walsh, 1998; Hood, 2007), rather than highlighting the exceptional, the focus was on patterns of everyday occurrence. In line with the generic inductive qualitative method, the focus in the themes was on identifying areas of "substantive richness" (Hood, 2007, p.161) rather than on developing theoretical categories. Where a pattern was identified, alternative explanations and negative cases were sought (Carspecken \& MacGillivray, 1998). Where a pattern was claimed as typical, notes were kept of how frequently it occurred (Hammersley \& Atkinson, 1995). As fieldwork progressed and themes emerged, coding became increasingly focused; this progressive funneling of analysis (Hammersley \& Atkinson, 1995; Hood, 2007) continued post fieldwork through the writing and revision stages. Records of coding decisions were kept (Carspecken, 1996) throughout the process.

While the quantity of data gathered made full-scale informant checking impractical, when chapters were finally drafted teachers had the opportunity to give feedback. In two cases, where particular children featured significantly, material relating to them was given to the family with the suggestion they share it with the child, and feedback was invited. The robustness of interpretations was strengthened by teachers' feedback, by peer checking for biases in interpretation with supervisors and academic colleagues, and by considering the interpretations within the context of similar recent New Zealand research (see Chapter 9, section 9.3).

Finally, this discussion of rigour needs to consider postmodern critique which questions the possibility of capturing the 'truth' in the interpretation. This critique recognises that the 'reality' presented can only ever be "a construction, one of many possible slices or images of reality" (Denzin, 1997, p.42). While there are both positivist and postmodern ethnographers (Pole \& Morrison, 2003), there are also ethnographers who draw elements from the postmodern critique, such as attention to multiple perspectives, and the avoidance of meta-narratives (Davies, 1999), but do not relinquish belief in the value of the approach (Pole \& Morrison, 2003). The challenge in this thesis was to find ways to capture the ambiguity and complexity. 
Using a range of children's voices was not only a way of giving credibility (Pole \& Morrison, 2003), but also helped to convey some of that complexity.

The third criticism of ethnographic research—whether a study of one context can be generalised to others- has been "hotly debated" (Hammersley \& Atkinson, 1995). The argument for generalisability or (more appropriately) transferability, in this thesis derives from the intention to highlight common practices and assumptions, rather than details unique to the context. My professional experience in the sector helped in making this distinction.

Because critical pedagogy contributed to the theoretical framework for the thesis, the tenets of critical ethnography warrant attention. The description of a critical ethnographer as one who studies culture for the purpose of changing it (Thomas, 1993) made me reluctant to adopt the label. Undertaking research with a foregone commitment to change not only suggests a presumptiveness about what will be found, but also may underestimate the difficulties of engendering change (Thomas, 1993). Here the intention was more modest. It was not to initiate change, but rather to encourage debate both in the immediate setting, and across the sector through professional networking, writing and teaching (Hammersley \& Atkinson, 1995). This thesis was critical in the sense of having the aim of "digging below mundane surface appearances ... to display a multiplicity of alternate meanings" (Thomas, 1993, p.5).

In the next section, discussion of the plan to develop strategies to use with children is set in the context of the strategies others had used in foregrounding children's perspectives.

\subsubsection{Identifying potential strategies to foreground children's perspectives}

Reviewing the literature to discover strategies others had tried revealed two recurrent themes: the need to address power differentials in research with children (e.g., Flewitt, 2005; Krieg, 2003; Sumison, 2003), and the right of children to respect (e.g., Birbeck \& Drummond, 2005; Hedges, 2002a; Sumison, 2003). More specifically, there is useful information on a number of strategies that have been used with young children, although few studies refer to research with children aged 3 (Clark, 2004, 2007; Einarsdottir, 2007; Farrell, Tayler, \& Tennent, 2002; Flewitt, 2005; Howard, 2002) and even fewer include children aged 2 or younger 
(Clark \& Moss, 2001; Einarsdottir, 2007). The informal interview, either for individual or small group, is a well-established approach. Discussions in the literature include the effectiveness of interviews with single children (Alldred, 1998), pairs and small groups (Carr, 2000; Mayall, 2000; Smith et al., 2005), and provide thoughts on the potential benefits of group interviews (Einarsdottir, 2007; Graue \& Walsh, 1998; Lewis, 2001; Te One, 2007) as well as their challenges (Dockrell, Lewis \& Lindsay, 2000; Hedges, 2002a). There are comments on the settings for talking with children, and the benefits of using a separate room (Dockrell et al., 2000; Nespor, 1998; Smith, Duncan, \& Marshall, 2005) or of talking with children while they are engaged in activities (Connolly, 1998; Te One, 2007). Drawing has also been used as a way of expressing ideas (Einarsdottir, 2007; Kennedy \& Ridgway, 2005; Wiltz \& Klein, 2001) in interviews.

Examples of the questions asked in interviews provided useful starting points for my own thinking. These include questions about what children like and dislike about their classrooms (Einarsdottir, 2007; Lewis, 2001); what they like doing best (Farrell et al., 2002; Wiltz \& Klein, 2001); why they come to the centre/school (Farrell et al., 2002); and about rules, and 'good' and 'bad' behaviour (Formosinho \& Araujo, 2004). In exploring children's engagement in learning Smith et al. (2005) have asked questions about why they were involved in an activity and whether the activity challenged them.

A variety of tools have been used by researchers. A partially completed book (Carr, 2000), a persona doll (Te One, 2007) and short scenarios (Te One, 2007) have been used as stimuli for discussion. Questionnaires have been used (Einarsdottir, 2007) and ingenious rating scales have been devised which involve children in activities such as rolling a piece of fabric across a scale (Dockrell et al., 2000).

Photographs have been widely used. Photographs of recent activities have been used as a focus in interviews (Smith et al., 2005; Wiltz \& Klein, 2001). Children have taken their own photographs of favourite places or activities in the setting (Clark, 2004, 2007; Clark \& Moss, 2001; Cremin \& Slatter, 2004; Einarsdottir, 2005, 2007; Greenfield, 2004) and the relative merits of disposable and digital cameras have been discussed (Einarsdottir, 2005).Video (e.g., Murfin \& 
Butterworth, 1999) and tape recorders (e.g., Wiltz \& Klein, 2001) have been used by adults, and children have worn microphones (e.g., Flewitt, 2005) and camcorders (E. J. White, personal communication, November 11, 2008).

As a result of the reading, and past experience, the following list of strategies to be trialed was developed:

- observing children, at times when they agree to it

- talking with children, either singly or in groups, as it happens within the flow of the session, or at times they nominate

- giving children the opportunity to take photographs within the centre as a way of identifying what for them are significant areas/equipment/people

- using photographs (taken by me, or by children) as prompts for conversations

- completing a questionnaire with individual children using smiling and frowning faces as a way of exploring what they consider they are learning in the centre

- audio-recording children's conversations

- child-led tours of the centre.

It was anticipated that a set of strategies that 'fitted' the children and the context would emerge.

In an effort to strengthen the commitment to foregrounding children's perspectives, and to 'interrupt' deep-seated patterns of thinking about the role of children in research, a set of checking questions was constructed before data generation commenced. These were referred to during the five months.

- Have I begun with children's thoughts?

- How can the ideas of children be included at this stage?

- What are the assumptions?

- What questions might a child ask?

- How can children engage with this topic in a way that interests them?

- How can I avoid children giving me the answer they think I want?

- How can I ensure children's own agenda is minimally disrupted?

- What are the power dynamics in this data-generating situation?

- How open am I to following the children's lead? 


\subsubsection{The six focus children}

Selecting to focus on particular children towards the end of the research period was a strategy suggested by a thesis supervisor based on the premise that some children would choose to participate more actively in the research activities, and that it would be useful to collect more detailed data on them. Initially, I referred to these as 'case studies', but having read in more detail about what defines a case study (Bassey, 2003; Burton, 2000) I opted to use the phrase focus children instead. It was decided to interview a parent of each of these children in order to gain insight into parent perspectives, to discover what children took from their centre lives into their home lives, what their out-of-centre interests were, and to provide a further level of triangulation. The decision about the number of focus children was left open.

\subsubsection{The plan for data gathering}

The planned combination of ethnographic observations, strategies focused on children's perspectives, a more in-depth focus on particular children and interviews with their parents- the mixed approach outlined above reflects the broad and relatively uncharted nature of the topic. While a plan of the stages of data gathering was prepared, this was considered to be flexible to allow for unfolding events in the centre or in the research to be accommodated. (See Table 1 for the plan of data generation.) In adopting this flexible mixed-method approach to data generation, in which methodological strategies would be used as they seemed appropriate, there was again an alignment with the concept of bricolage, of "using a variety of tools as they become necessary in the research process, rather than passively receiving the "correct" tools" (Kincheloe \& McLaren, 2005, p.316) The commitment here was to use the planned methodology as a guide, and in any situation to attempt to find the strategy that best fitted that particular context and child/children. 


\section{Plan for data generation}

\section{Pre data gathering}

Initial visits to the centre to allow children and families to meet me, as they decide whether they wish to participate in the research.

\section{Stage one}

During the first days gather information about the planned teaching/learning currently occurring through document analysis of:

- children's portfolios (records of children's learning kept by teachers, and available to children and their families),

- teachers' planning documentation,

- centre newsletters.

(It was anticipated that data from these sources would show the planned teaching/learning currently happening within the setting, and provide a framework for the rest of the research.)

Gather detailed information on the centre environment and the resources for children.

(Beginning with a focus on the environment was seen as a non-threatening way to establish the researcher role and to build rapport with children.)

\section{Stage two}

As rapport is established, the collection of detailed ethnographic fieldnotes will begin, and alongside this the other strategies for talking with children will be introduced and developed.

\section{Stage three}

Towards the end of data collection, identify focus children who have been particularly interested in interacting with the researcher. This will be done with the knowledge of the teachers and parents, and will use the research approaches developed in stage two. A semi-structured interview with a parent of each of these children will be arranged to gather additional information. 


\subsection{The human dimension}

This section outlines the process of identifying and negotiating access to the centre, establishing ethically based relationships with children and adults, and the day-to-day challenges of maintaining those relationships.

\subsubsection{Locating and gaining access to a centre}

Once approval was gained from the ethics committee of the School of Education, Victoria University in April 2005, the process of selecting a centre began. A list of criteria had been drawn up:

- A mixed-age childcare centre that caters for children from 2 to 5 years, although younger children could also be on the roll

- Full-day licence

- A recent history of low staff turnover

- No recent history of management difficulties

- Community-based (i.e. not privately owned)

- Relatively experienced staff

- Within $15 \mathrm{~km}$ of my home.

Following the lead of Nuttall (2004), two colleagues with professional knowledge of the sector were asked to suggest centres meeting those criteria. Reports from the Education Review Office (the organisation monitoring the quality of all New Zealand schools and early childhood centres) were consulted for centres named by both colleagues, and evidence that high standards of quality and care were offered was key in deciding which centre to approach. An informal approach to the supervisor was greeted with enthusiasm, and the formal process of negotiating access commenced. Once the permission was granted by the management committee, consent forms were given to adults working in the centre, then introductory visits to the centre began in late July and consent/assent forms for the children and their families were distributed. Each family and teacher received an information sheet about the study (see Appendix 1), a letter giving information about confidentiality and the right to withdraw, and a form to complete indicating willingness to participate. Each family also received an assent form for each child with the request they help their child/children complete the form if they judged their child capable of making the decision (see Appendices 2-6 for permission letters and consent forms). Initially consent/assent forms were only supplied for children aged 
2 years and over, but at the request of families, they were provided for all children. With hindsight I recognise the original decision was based on age-related assumptions about competence which were at odds with the tenor of the research.

At the start of data generation there were 37 children on the roll, both part-time and full-time, and 36 children participated in the study. One child's assent form refused participation, one family's consent form was not returned but a 3-year old girl who started at the centre during the process joined the cohort. One very young child who started late in the process was not invited to join. Care was taken that no one should be aware of who was not part of the research; they were always included if they chose to be but no information was recorded.

Of the 36 children in the research, 16 were girls and 20 were boys, and there were four pairs of siblings. Ten children aged under 2 were included, five of whom turned 2 during the data-generation period. There was a core group of 20 who attended either four or five days a week. This included six of the nine boys aged 3 or 4 , and three of the eight girls aged 3 or 4 . Six of the children under 2 attended five days a week. Two children came only once a week. The composition of the group changed during the period. Three girls turned 5 and left for school, two boys left shortly after the research began, two brothers left when their parents shifted, and two other boys (one aged 2 and one aged 3 ) left towards the end of the period. The days/hours of attending for some children also altered. The fact that when one teacher left during the research, her position was covered by the other teachers increasing their hours contributed to the cohesiveness of the community. The children were predominantly European New Zealanders, but their cultural backgrounds included Māori, Samoan, Tongan, Tokelauan, Fijian, Chinese, South African, and Greek.

The decision potentially to include every child meshed with the commitment to children in the research. While researchers often choose to work with a selection of children (e.g., Clark, 2004; Greenfield, 2004, 2007) such a decision would not only have raised equity issues, but would have been at odds with the complexity assumed to be inherent in the research questions. Although six children were identified towards the end of data generation in order to provide a depth of data about particular individuals, all children still remained important. The most significant difference was that a parent of each of those six children was interviewed. 
The six focus children, with their ages at the start of the data generation, were:

Aidy, male, European NZ, 3 years 6 months

Cassidy, male, European NZ, 2 years 7 months

Evie, female, European NZ, 3 years 10 months

Fleur, female, European NZ, 2 years

Rex, male, Māori/Tokelauan/European NZ, 4 years 1 month

Robert T, male, Māori, 2 years 4 months

The nine women working in the centre-seven teachers, a manager and a cookall participated in the research. Two teachers held Bachelor of Education (Teaching) Early Childhood Education degrees, one had a Bachelor of Education in primary teaching, two had Diplomas of Teaching (Early Childhood) and two were completing that Diploma's final year. The staff ratios were 1:4 for children under 2, and 1:5 for those over 2. The teaching supervisor had worked in the centre for six years, five teachers had worked there for at least three years, and the seventh teacher had joined the team earlier that year. One teacher left and her position was covered by the others increasing their hours. The manager and cook had both worked there for a number of years. The cook's interactions with children were limited to mealtimes but the manager's role brought her into the play areas more frequently, and occasionally, when a teacher was absent, she filled in.

In light of the focus on children's perspectives, it was decided not to name individual teachers but it must be acknowledged that the diversity and richness of the teaching team, and the unique pedagogical and personal style of each teacher, are concealed as a result of that decision. However, experience suggested naming the teachers and differentiating between them, would inevitably allow the focus to slide to the adults. Therefore, any adult interacting with a child has been called 'teacher', the term preferred by children. The cook and manager have been referred to as 'an adult'.

\subsubsection{Establishing and maintaining ethical relationships with children}

Discussion concerning ethical dilemmas of research with young children (e.g., Cullen, Hedges \& Bone, 2005) highlights the need for informed consent before and during the research and the need for protection during and beyond the research (James et al., 1998). My focus on respecting children's rights and 
maximising their control made it essential they were part of the consent process (Fasoli, 2003; Nespor, 1998). Following suggestions in the literature (Bone, 2005; Hedges, 2002a), an assent form for children was designed. Twenty-one children completed assent forms, including one who chose not to participate.

The children's assent form and the letter to parents both stressed children could withdraw at any stage. This was taken to mean:

- any indications, verbal or non-verbal, that they do not wish to be observed or to participate will be respected,

- the families and teachers will inform me if any child /children shows unease about the research or about my presence in the centre.

While it is easy to assert that children are entitled to the same degree of protection, confidentiality and anonymity as adults, in practice this is not simple. Grappling with the tensions between the rights of children to confidentiality and the rights of families and teachers was challenging. It was decided that transcripts of conversations with individual children would only be made available to teachers and families if children agreed. The families of children who were considered too young to give informed assent would be able to see all the information relating to their child on request. Copies of photographs of children would be made available to teachers and families if children agreed, or if children initiated this process. Photographs would not be included in the thesis or in presentations arising from it as this would breach children's confidentiality.

Another ethical issue related to how to respond to examples of prejudice, and to avoid compounding injustices (James, 2005). MacNaughton (2003a) warns of the danger of researchers unwittingly colluding in children's marginalisation, particularly in relation to race, class and gender, and argues that "Bringing forth children's voices is indefensible if those voices merely serve to silence some, marginalize others, or reinforce unjust ways of becoming” (p. 42). Her list of questions to be asked before including children's voices in research challenged me through the thesis process:

- which children's voices will come out of it?

- what will be the consequence for each child who participates?

- how might one child's voice silence that of another? 
- what can and should I do when the voices, I and others hear, are racist or sexist?

- how might intervening in one child's voicing of their knowledge enable another child to speak?

- how will I honour those children whose voices struggle to be heard? (MacNaughton, 2003, p.41)

Once data collection began, inevitably tensions emerged between the research role I planned to take, the ethical commitments I had made, and the realities of day-today centre life. Although accounts by other researchers (Corsaro, 1985, 2003; Hadley \& Nenga, 2004) were valuable in defining the role to take with children, I found in reality my roles were multiple and shifting, and that a number of factors contributed to the ambiguities. One was that I felt impelled to support the teachers. Sometimes this meant tidying a room, but at other times it influenced my interactions with children. For example, when a child chose to sit on my knee for mat-time I would "shh" them quietly if they talked. More contentiously, I realised during the first weeks of data gathering that I found it ethically challenging not to react when I was the only adult who saw children physically hurt each other. MacNaughton's (2003) challenge to consider how one should respond to inequitable practices sat with me, and I began to react with a look or brief comment in these situations, but I still find this aspect of the role problematic.

My starting point for engaging with children was respect. I asked children if I could watch them, and invited children to participate in activities with me as and when they wanted. I tried to be sensitive in timing requests to observe children, and no child refused. I asked children's permission to observe or tape-record their play, and when occasionally the changing situation meant I recorded children without their permission, I later explained their voices had been taped, let them listen, and asked their permission to type it up. I tried to be sensitive to children on the sidelines, and invited them to participate, and I carried two notebooks and pens so there was a spare book and pen; these were particularly attractive to younger ones. With the teachers' knowledge, occasionally I invited non-sleeping children to spend the 'quiet time' after lunch with me in a side room off the main playroom. This allowed me to have uninterrupted time with up to three children on several occasions.

Alongside the efforts to establish rapport with children, there was also a need to maintain detachment. This was not only inherent in the methodology (Pole \& 
Morrison, 2003) but I wanted to ensure I did not become too significant a figure for any child, given my limited time in the setting. While the power relationship between researcher and children is frequently described, only a few (Nespor, 2003; Smith et al., 2005) describe children's wielding of power within these interactions. Such references were reassuring as my interactions with two of the older girls, Maxine and Grace, were occasionally characterised by power. They took my pencil saying they would not give it back, defined me as the 'baddie' when they made swords, and when I wore black announced it was "yucky". These sound insignificant but in the moment I was aware of the latent power they were wielding; yet on many other occasions they invited me to sit with them, or moved to join me in the centre. These experiences were a useful reminder of how their peers might feel when such comments were directed at them.

\subsubsection{Establishing and maintaining a research relationship with the adults}

Although no data were to be gathered formally from the nine adults, I understood the potential impact of a researcher in the centre. My role with the cook was limited, but the manager had a pivotal role in the centre, and therefore there was more contact with her. My role with the teachers was a complex relationship characterised by a series of tensions. The first tension was the challenge of juggling the ongoing balance between research and reciprocity. I was deeply indebted to these women who had allowed me into their workplace. When I was aware teachers were under pressure, I tried to help out. I also sometimes brought food for morning tea, attended social events and joined in the Saturday morning spring-cleaning. I tried to be sensitive to their perspective, and to fit my research agenda around their routines. A second tension arose from my awareness of the support they provided for me. They understood I was avoiding discipline situations, and at times I knew this led to their intervening on my behalf. While I modified the stance on non-intervention, even towards the end of the five months there were still occasions when teachers stepped in. A third tension was that teachers did not have a voice. While the methodology foregrounded children's perspectives, teachers were also under the spotlight, which placed them in an invidious position. I warned teachers at the beginning that I would always be in 'observe' mode, and encouraged them to clarify when a conversation was 'off the record'; this was rarely requested although there were many conversations, both casual and professional. A final tension has arisen as I have begun to present findings from the research at professional gatherings. Among the early responses 
there have been some critiques of the centre. While no centre is above criticism, to focus on this single setting is to misunderstand the broad intent of the research. McCadden (1998), describing a similar tension between critiquing the setting and acknowledging the teacher, wrote "The resolution of this tension permeates my narrative" (p. xviii). If what I write is seen merely as a comment on the practices of these teachers I will have failed in my intent to reflect on assumptions and practices that are sector-wide. As well as informal expressions of gratitude through the data gathering each of the nine adults in the centre received a personal letter at the end of this period, and I also chose to give the centre a colour printer to complement the digital camera they had purchased.

\subsubsection{Establishing and maintaining a research relationship with the families}

Making myself available in the centre during the first few days meant I established early links with many families, and teachers were proactive in introducing me to others during the following weeks. Attending centre social events such as the fish-and-chip evening provided further opportunities for communication. Each family received a series of newsletters describing progress in the research during and after the completion of data generation, and children received their own versions. Once the final draft of the thesis was complete, a written summary of findings was given to families who had requested it. Each child was given a folder containing a few coloured prints of them that they could take home; children who left the centre during the research period received a farewell letter, and others received one at the end of the period.

Towards the end of the research period, a parent of each of the six focus children was asked if they were willing to be interviewed, and all agreed. Interviews were semi-structured, focusing on their child's interests, and how they talked about the centre at home. All parents interviewed were mothers, and all chose to have the interview at the centre, one chose to talk in the staff room, but the others chose to talk in the play areas, which allowed their child to contribute to the conversation if they wished. A teacher chose to participate during part of the interview in the staff room. 


\subsection{The reality of the research}

This section describes the changes made in the methodology as it was implemented. Described retrospectively, the account seems straightforward, but the lived process had many moments of quandary.

\subsubsection{Honing the research questions}

As explained in Chapter 1, the original research question was refined. This occurred after data collection was complete, and a first round of analysis and writing had been undertaken. The process had shown how very different each child's experience of curriculum might be, and therefore the enormity of attempting to convey the scope of every child's experience. The breadth of the question had produced an unwieldy spread of data and wide-ranging rather than deep analysis. The dilemma was to find a way to tighten the topic so a more cohesive and focused story could be told within the constraints of the thesis. To choose to focus on just a few children was rejected because it ran counter to the commitment to include all children and would have concealed much of the complexity of their collective experiences. The other option was to narrow the research question. To consider only the boundaries of curriculum would still allow both overt and covert aspects to be included, and would retain the focus on the perspectives and experience of all the children. Reassuringly, analysis showed that the central themes that had emerged in relation to the original question were replicated within this narrower analysis. The central research question was therefore narrowed and rephrased as:

What are the boundaries of the curriculum in the early childhood setting, and how do young children experience, influence and enact these boundaries?

The subsidiary layers of questions were also amended (see Figure 3 for the final research questions) and a second process of simultaneous re-analysis and rewriting was undertaken. 


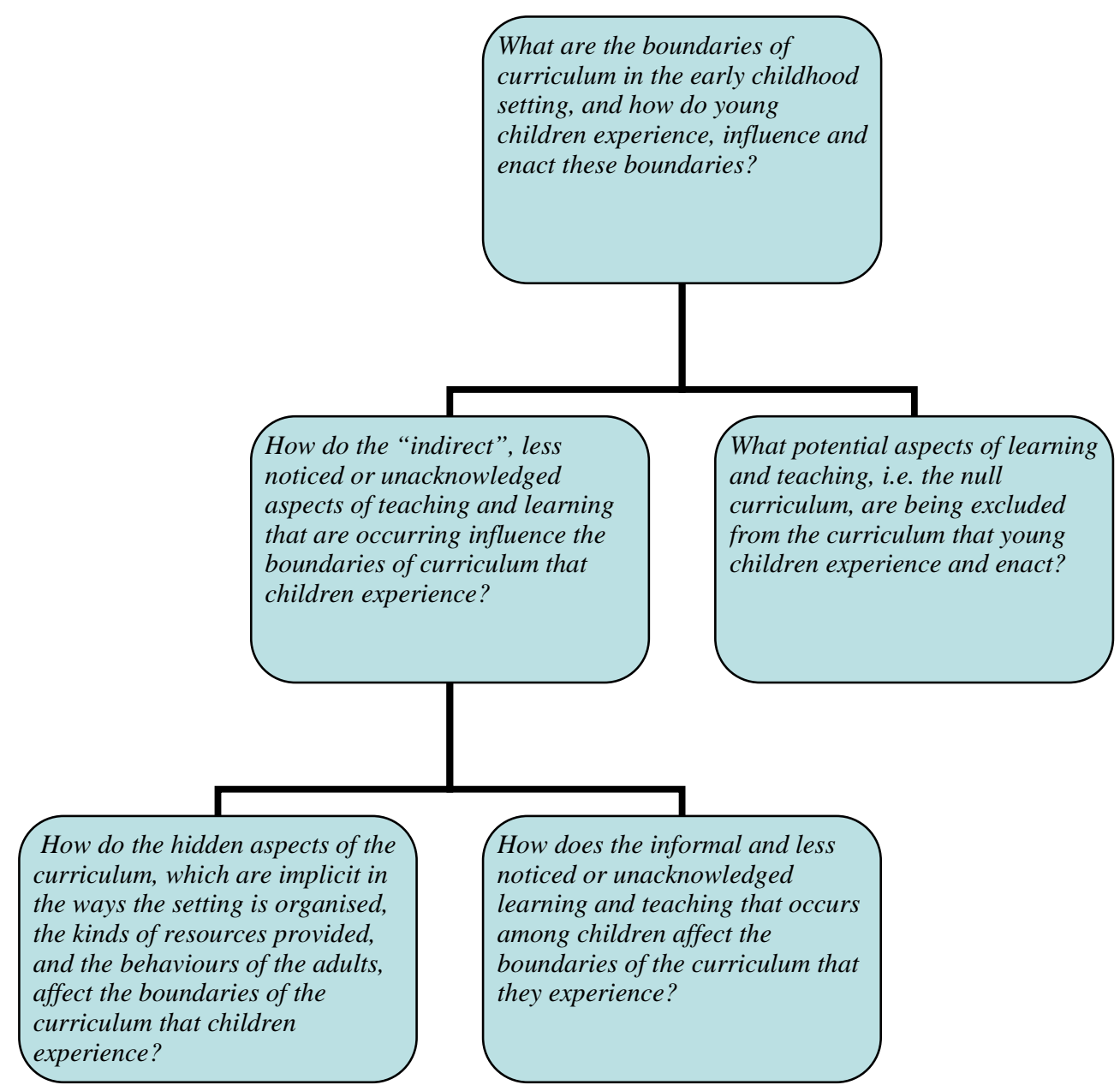

Figure 3: The final research questions

\subsubsection{Changes in data generation}

While there was a clear plan for data generation (outlined in section 4.2.4), the reality of the process was less straightforward. This section describes the reality of data generation and the development of the strategies that were used with children. The first visit was in mid-July, and the final visit was in mid-December. There were 16 weeks of data gathering with three periods of a week or more when I was not available, which provided opportunity for reflection and refocusing.

Altogether 50 visits were made.

There were some changes to the data-collection plan. While it was intended to transcribe a range of documents during the first weeks, in fact this happened later. Once the consent/assent forms were returned building relationships with children seemed a greater priority. However information on the environment was still collected in this first stage. A second change related to the selection of focus children. The original intention had been to select children who had developed a particular rapport with the researcher, but the final selection was guided more by 
emerging themes, and by ensuring there was a representative balance of younger/older, male/female, and ethnic background; this concern with "demographic "representativeness"” (Hood, 2007, p.154) is seen as a feature of the generic inductive qualitative method. A further change was that the notebooks of the six focus children were transcribed. These books, which provided a communication link between teachers and families, contained learning stories, and comments written by teachers and occasionally parents. (They were phased out towards the end of the study as part of a review of programme planning procedures.) Other additional documents transcribed were: the notices written by teachers that were displayed on the walls, and the daily whiteboard messages for parents. Other displayed material, such as Ministry of Education notices, were noted but not transcribed in full. Other aspects of data generation occurred as planned.

\subsubsection{The development of child-focused strategies}

Throughout the study, alongside ongoing observations, a range of strategies was developed focusing on talking with children about their centre experiences. Many children were keen to spend time with an interested adult and so it became a search for ways of talking with them that they would find engaging, and that would allow them to articulate their ideas. This study reiterated the usefulness of offering children a range of ways of expressing their ideas (Clark, 2004, 2007). Not only was it possible to design a process that was sensitive to the context, but it was also possible for each child to select within this range.

\section{Strategy 1: What children call this place?}

Using a folder of photographs of sites within the centre as a stimulus for conversations was the first strategy used. It was initially intended as a focus for conversations as part of the process of getting to know children and finding the names they used for places, but proved useful in other ways. The photographs included adult spaces like the office and kitchen, as well as indoor and outdoor play areas. Conversations with children showed which areas were familiar, the names used for each area, and suggested what might be important to them about the different spaces. 


\section{Strategy 2: Child-led photo tour of the centre}

The strategy of children taking photographs using a digital camera was planned as a way of exploring children's thoughts about the environment, but the interactions plus the photographs generated a far wider range of data. As with all the strategies, the process evolved. The format was that a child was invited, or volunteered, to show me the places they liked around the centre. They could either take the photographs, or be photographed in places and with people they nominated. Having noticed that the first children chose to move outside to take their initial photographs, the camera was always given to the child indoors, and tours were only done when both indoor and outdoor environments were accessible.

\section{Strategy 3: Photos and stickers > photo chart and counters}

Photographs of activities in the centre were used to explore children's reactions to activities/events in centre life. These included events such as mat-time, lunchtime and sleeping, as well activities such as puzzles, blocks and sand play. At first these were presented as a set of photos, but they proved cumbersome so they were converted into a single chart of 30 small photos. Initially children were given five coloured dots to stick on those they liked, but this limit was clearly unsatisfactory for them. However, when the number of dots was increased, they were put on every image. When the chart was developed, coloured counters were used, 20 with happy faces drawn on them, and five with sad faces, which required children to make choices. This research strategy was enjoyed by a wide range of age groups. While it generated a lot of data and was the setting for interesting conversations, the way some children undertook the activity (wanting to cover every photo) led me to doubt its usefulness in defining liked/disliked events and activities.

\section{Strategy 4: Picture questionnaire}

The questionnaire allowed children to draw answers to questions as a way of increasing their control. Because the questionnaire included relatively sensitive questions, for example naming children they did not like to play with, this was only used with individuals or pairs, but it generated interesting conversations. This was the research strategy I felt most tentative using, aware of the dilemmas of exploring children's friendships (James, 2005), and perhaps not wanting to allow children to openly express rejection of peers, despite the fact that observations showed me exclusion and rejection did occur. 


\section{Strategy 5: Sharing learning portfolios}

Children were invited to show me their learning portfolios. This was initiated because I wanted their permission to look at these documents, to see what aspects of learning had been included, and to find what particularly interested them. The first children asked were uncharacteristically reluctant and so a strategy of taking photographs of the pages each child saw as significant was introduced. A large number of children, including some as young as 2, chose to show their portfolios. While this strategy did not generate the conversations about learning that had been anticipated, the interactions proved valuable.

\section{Strategy 6: The New Kid book}

In order to explore children's ideas about learning and teaching in the setting a 'New Kid' book was developed. The storyline was that a new baby came to the centre, watched the other children, and asked children and teachers to teach/show him/her how to do things, so he/she could be like them. This was used with a number of children between 2 and 4 years. Although this strategy did not produce many examples of children using 'teaching' or 'showing', discussing the book led to thought-provoking conversations.

\section{Strategy 7: The Naughty book}

The 'Naughty book' was developed to explore children's ideas about what constituted inappropriate behaviour in the setting. The storyline was that a child spent the day at the centre doing naughty things, which the children were asked to specify. (The next morning the child woke up covered with spots which the mother saw in hindsight as an explanation of the behaviour.) Unexpectedly, many children seemed to find it difficult to offer examples of inappropriate behaviour, so this strategy was not often used.

\section{Strategy 8: Informal conversations}

While informal conversation lacks the novelty of some of the methods outlined above, conversations with children within the flow of centre life contributed significantly to my awareness of children's perspectives. Informal conversations were a constant feature of being with children and ran through all the above strategies. At times children initiated conversations and at times I did. Mealtimes provided opportunities for group conversations; play contexts were more often the setting for one-to-one interactions. On occasions, conversations with both groups and 
individuals were used to ask 'research' questions. For example, on several occasions during informal conversation I asked children about why they came to the centre.

\section{Strategy 9: Observations}

Observations were the matrix within which the other strategies occurred.

Sometimes this was observation as it is traditionally known-writing notes, taperecording and taking occasional photographs. Sometimes it was playing with children, but taking mental notes which were recorded as soon as practical. Sometimes it was sitting in on routines of centre life-mat-time, meals, tidying up, sleeptime. In these situations, brief notes were made at the time, and detailed records typed up later. Initial observations were broad, with no particular focus, but later observations focused on events, on particular children, on activities, and on emerging themes. The luxury of being present with children, with no teaching duties, contributed to the depth of rapport established. The bulk of the data came from these last two strategies, and the understanding of children's perspectives gained here frequently illuminated data obtained through the other strategies, and justified the decision to spend a prolonged period in the centre. (See section 9.2.3 for a discussion of data generated with each strategy.)

These strategies have been described in detail as part of the process of including the researcher lens/eye within the scope of the research. While each was designed for a purpose and to fit the context, each also reflected assumptions/expectations on my part.

\subsection{The management and analysis of the data}

This final section describes the data collection and analysis. That process of analysis began with the choice of research topic, theoretical perspectives and methodology and with the framing of research questions. Analysis continued in the reading I chose to do, and the details I recorded from that reading. While not often described by researchers, Pole (2003) uses the phrase anticipatory data reduction to describe this part of the process.

Once data generation commenced, analysis of the information gathered about the environment began immediately, but other coding did not begin for several weeks. As data were collected written notes and audio-tapes were transcribed before the next visit. All written data were typed in a format suitable for entering into the 
qualitative data analysis programme QSR N6. Data gathered through observations and interactions with children were identified by date, e.g., [18/2/05]; data that came from centre planning documentation were identified [Pr...], and material taken from individual children's notebooks was identified [Nobk...]. Transcribing data provided an opportunity for reflection, and initial coding thoughts were recorded as memos at the end of each transcription. This had the advantage of linking them to the context in which they arose. As part of this process, a focus was set for the next visit, although these were not always realised. The concomitant process of ongoing reflection and analysis, of "moving back and forth among questions, data gathering, and data analysis" (Hood, 2007, p.156) which is characteristic of the generic inductive qualitative model, generated leads. By the start of 2006 all data were transcribed and had an initial coding. (See appendix 7 for coding categories.)

Photography was used to record the planning documentation (six-weekly planning, children's notebooks, portfolios) which meant documents were not removed from the centre. Transcribing documents from these photographs was laborious and done more slowly. Photographs of the environment, activities, and events were taken as an adjunct to fieldnotes, and were drawn on in typing up each day's data. These were then filed electronically by date making it easy to revisit them.

Having used piles of cut-up paper in analysing previous research, it was liberating to use a computer programme for coding and the first stages of analysis. The flexibility of the programme made it easy to change coding decisions, to code data in multiple ways, and to search the data. It was also possible to keep records of coding at different stages. While the robustness of the analysis still rested on my coding decisions, the programme made data-handling very easy. However, the final and most detailed levels of analysis occurred alongside and as part of the writing and revision process. While the coded material provided an excellent platform for this final stage, I found that I resorted back to using pen and paper at times. For example, I tallied children's choices of favourite activities, from strategy number 3 , but did not code these data.

The process of analysis was a layered one. When coding was commenced, initial categories used were "manifest" (Berg, 2004, p. 270), close to the reality of the 
research in the centre. For example, data on each participant were entered under their name, and data generated by each research strategy were entered under that label. As data analysis proceeded, the intent was to identify themes rather than to develop theoretical categories leading to a formal theory (which a Grounded Theory approach would have required (Hood, 2007)). As possible themes began to emerge the initial coding for them was intentionally broad and derived from the research questions to avoid the danger of too quickly defining the pattern of analysis. For example, a code of 'Null curriculum' was used. While 'being powerful' and 'being part of the community', and the 'adult-child division' emerged very early as significant aspects of the data, I continued to search for other patterns. As these themes continued to strengthen, I consciously broadened my scope and looked more widely, for connections between patterns (Pole \& Morrison, 2003), for incidents that shed doubt on my research hunches, for negative cases in the data, as well as for the similarities in what I was seeing and hearing. Coding became increasingly focused during this process. For example, as the concept of children 'being powerful' emerged, a set of codes was developed that explored the ways in which this was displayed: children being powerful, boys' power, older and younger, children's use of rules, being powerful with adults, being powerful with me. A similar but more extensive set of codes was used for exploring how teachers expressed and reacted to expressions of power. Even when episodes seemed to be replays of familiar material, I recorded and transcribed them recognising that one aspect of rigour is indicating frequency of occurrence (Pole \& Morrison, 2003). Towards the end of the five months, the process of coding and analysis took increasing precedence and continued long after visits were completed. The full data set was revisited multiple times through the progressive process of analysis. Both taking the perspectives of children and considering what the implicit messages might be (two of my three strategies of dislocation) proved useful in uncovering assumptions.

Once data-collection was completed, a first round of simultaneous writing and analysis was undertaken, and the findings chapters were drafted. It was only at the completion of this period of sustained writing that the decision was made to refine the research question, and to focus only on the boundaries rather than attempting to consider both the scope and the boundaries of the curriculum. While the broad analysis decisions that had been made up to this point were still relevant, the changed focus meant detailed re-visiting of the data, and substantial revision of 
the drafted findings. The final layers in the process of analysis came with this rewriting, and coding decisions at this final stage were not added retrospectively into N6. The successive drafts of findings chapters (up to 15) record evolving analysis, and the 400 plus recorded text searches of data are indicative of this process.

Through the writing stages, the concepts from activity theory also became important. During the process of data generation they had, in their role as sensitising concepts, guided my attention at times; for example, awareness of the concept of rules, routines and rituals heightened my interest in these aspects of centre life. However, they had not been allowed to limit the evolving focus of the observations. During the writing process, structuring the final aspects of analysis around these three concepts, using them as successive windows through which to view the data, provided a last strategy for disrupting my assumptions. Once the final analysis was completed and the chapters were drafted, teachers were invited to comment on these, and families received a summary of findings with an opportunity to comment. Emerging thoughts and interpretations from the work were discussed with academic colleagues, with supervisors and others (Stephenson, 2008). Feedback from all these sources enhanced the interpretive process.

This chapter has reviewed the planned methodology, and described the reality of implementing those plans. The next four chapters present the findings. 


\section{CHAPTER 5}

\section{HOW THE PHYSICAL ENVIRONMENT INFLUENCED THE BOUNDARIES OF CURRICULUM}

The hidden curriculum is not something that we must look behind or around in order to detect; in most cases it is plainly in sight, and functions effortlessly. (Gair \& Mullins, 2001, p.23)

The first section of this chapter contains a description of the centre layout, the daily schedule and the detail of a cycle of planning in order to provide an indication of the current scope of curriculum at the start of data generation. This establishes a context against which to consider the findings presented in the remainder of this chapter and the following three chapters.

The sensitising concept which framed the analysis in the following sections of this chapter was the centre's physical environment and resources. The aim in foregrounding these less-questioned aspects of early childhood education was to consider how they influenced/constrained children's experience of curriculum, and how children reacted to those constraints. This chapter, plus the following two, build the answers to the following aspects of the research question:

- How do the hidden aspects of the curriculum, which are implicit in the ways the setting is organised, the kinds of resources provided, and the behaviours of the adults, affect the boundaries of the curriculum that children experience?

- What potential aspects of learning and teaching, i.e., the null curriculum, are being excluded from the curriculum that young children experience and enact?

The first of the two emergent themes of the thesis will be introduced. I will argue that the the physical location, architecture, and resources of the centre embodied and embedded a demarcation between children and adults which was the fundamental source of boundaries to children's curriculum experiences. The categorisation into adult and child seems brazenly self-evident, but as others have shown the obvious and everyday is often potent. Gair and Mullins (2001) describe the hidden curriculum as "not something that we must look behind or around in order to detect; in most cases it is plainly in sight, and functions effortlessly" (p.23). Here it seemed this categorisation was "plainly in sight" but as the layers of associated assumptions and implications were uncovered, it increasingly 
seemed this demarcation was a central source of curriculum boundaries. As the literature review in Chapter 3 showed, recognition of the adult-child demarcation is not novel (Brennan, 2005; Singer, 1996). However, the analysis in this chapter will contribute new insights in revealing how typical features of a centre environment can embody and embed that exclusion even within the setting.

While this analysis pertains to a single centre, personal experience as a teacher educator frequently visiting centres suggests this finding would be replicated in many centres, which indicates the demarcation between adult and child constitutes a 'regime of truth' which limits the ways we 'do' early childhood education, and the possibilities we envisage. Although the sociocultural approach of Te whäriki and the commitment to learning as socially constructed have challenged aspects of this regime of truth, the findings in this setting indicate the continuing strength of those familiar older traditions.

Mounting such an argument feels like a step onto treacherous ground because it could be read as risking much that early childhood education values, and particularly the commitment to play. At worst it could be read as a denial of the distinction between adults and children and as a first step back towards the evils of child labour. However, the intention here is not to deny the differences between 1-year-olds and 21-year-olds. Rather the aim is to argue for a blurring of the categories of 'adult' and 'child' and a re-examination of what Smith and Taylor (2000) call the "balance between agency and dependency" (p. 4) in the work of early childhood education.

The way in which the centre's architecture and resources embodied an adult-child demarcation is described in section 5.2. Two further concepts that emerged are introduced in sections 5.3 and 5.4: the significance of power/control and the importance of relationships and being part of a community. These two threads will be brought together in a second emergent theme to be introduced in Chapter 6 .

The data for this chapter included teachers' planning documentation, fieldnotes, photographs, semi-structured interviews with six parents, and children's conversations both informal, and in the context of three of the research strategies. These were the folder of photographs of centre places (Research strategy 1), childled photo tours (Research strategy 2), and the identification of liked/disliked activities (Research strategy 3). (See section 4.4.3 for descriptions of these 
strategies and section 9.2.3 for a discussion of their use. Parent interviews are described in section 4.3.4.) Coding references are given for longer data excerpts; to avoid making reading cumbersome, passing references to data in the flow of the text are not referenced. Where the source of the data is not fieldnotes of observations, the data source is noted in the text. Coding references 'Pr...' indicate centre programme planning documents, 'Nobk...' refers to children's notebooks.

\subsection{The current scope of curriculum}

In order to indicate the current scope of curriculum experienced, and enjoyed, by children and teachers in the centre, following a description of the centre layout, the format of a typical day is described, and the planning documentation for the initial six-week period is summarised.

\subsubsection{Centre layout}

Children and parents entered the centre building (see Figure 4) through the front door which led first to the foyer, and then to the locker room.

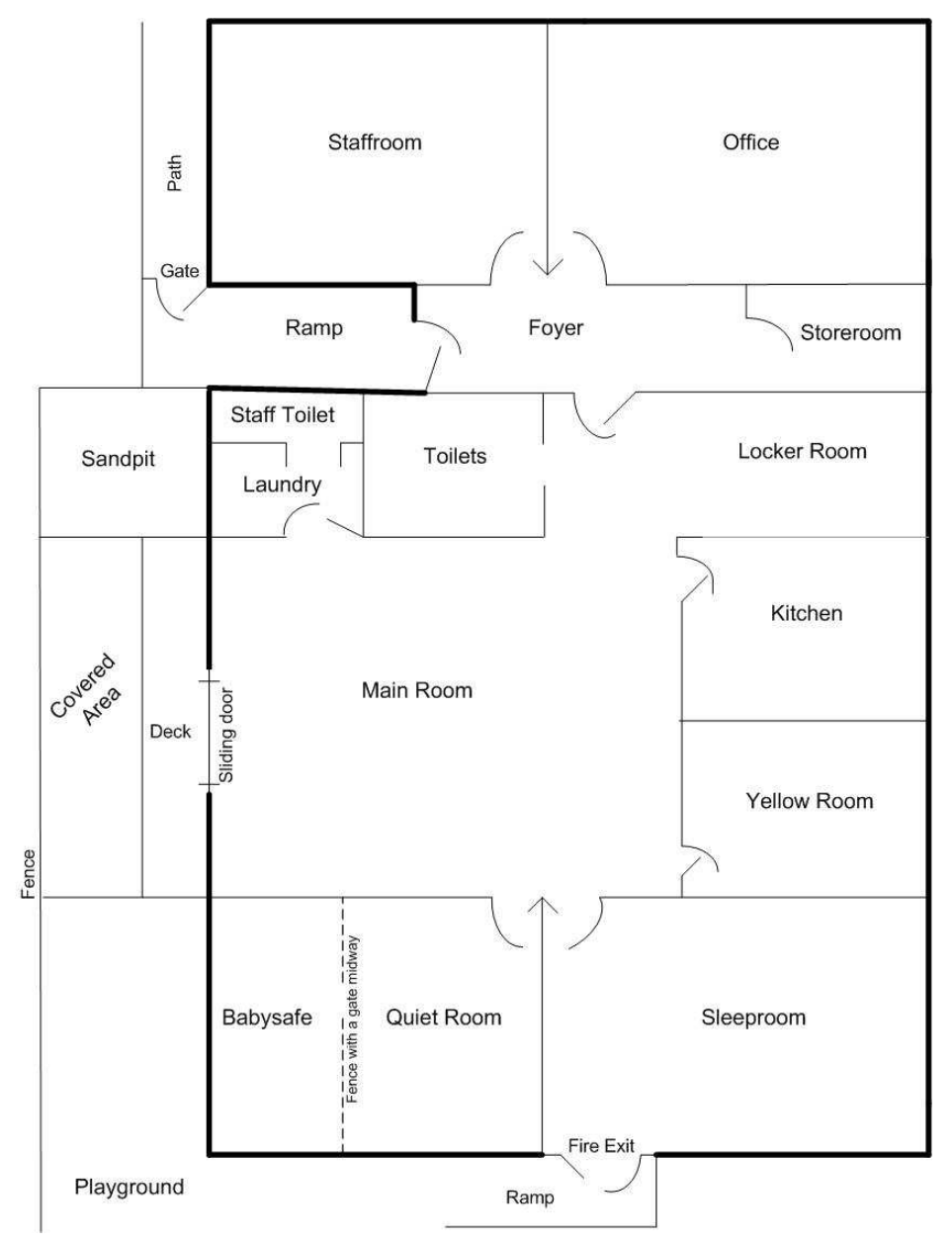

Figure 4: Centre plan 
Three other doors from the foyer led into the staffroom, the office and the storeroom. The children's bathroom and the main playroom were accessed from the locker room. The main playroom was the hub of the centre and often abuzz with activity. Leading off it were: the kitchen, the small yellow playroom, the sleeproom, the 'quiet' playroom, and the laundry which accessed the adult toilet. (Section 5.2.3 includes a list of the resources for children in each area.) The quiet room was divided by a low picket fence which made one end into the 'Babysafe', an enclosed area for the youngest children, which contained a range of toys and cushions. A gate allowed children access to this area.

The deck and surrounding roofed outdoor area, which contained the sandpit, were reached from the main playroom. On wet days, a heavy plastic wall was unrolled to enclose this area, but when the weather was fine children were free to use the whole playground which gave them access to the swings, the large wooden climbing structure (the fort), the two-child car set in the area surfaced with bark chips, and the areas of concrete and safety surfacing which were used for activities such as challenge courses, bike-riding and waterplay.

\subsubsection{A typical day}

What follows is a description, drawn from many days of observations, that captures the feel of a typical centre day. When the first children arrive at 8 am, the main room is welcoming; the three round tables are set up_-perhaps with purple sparkling playdough and rolling pins on one, coloured pencils and paper on the second, and puzzles on the third. The open mat area is inviting, edged with low shelves containing the musical instruments, wooden blocks, and crates containing Duplo and the wooden railway. The fish tank and nature table in the corner provide another point of interest. Displayed artworks and the teacher-made and commercial posters contribute to the colourfulness of the environment; when there is a new poster - a transport poster, a teacher-made poster of children gardening - groups cluster. Often a teacher is reading stories to children who are settling into their day. Arriving parents and children are greeted warmly, and there is a strong sense of rapport in the easy-going child-parent-teacher interactions. Sometimes children have something to show teachers-Charlie shows photographs of their new baby.

As more children and teachers arrive, other spaces are opened. By 8.15 am the door to the quiet room may be open; the yellow room door might open a little later. The 
weather, children's wishes, and the staff roster all influence when the doors to the outside are opened. Children often seem keen to be outdoors:

James is roaming — making noises, carrying two pieces of Duplo. [9/8/05]

He listens while a teacher reads a story, but says "Yeah!" when she finishes and quickly lines up at the door with others waiting for it to be opened. In cold/wet weather access to the enclosed deck area may be delayed, but in summer children may have access to the full playground from 8 am while equipment is still being set up. Along with regular provision of balls, ride-ons, bikes, swings, climbing equipment and sandplay, teachers often create spaces that invite imaginative playan enclosure built of tyres against the wall of the ramp with fabric stretched above to form an awning, a small tent pitched on the grass, the old kitchen equipment set up at one end of the sandpit.

By 9 am most children have arrived; latecomers may have little chance to play before the morning tea routine begins. Morning tea is often announced outside by a child calling from the deck. Teachers repeat the call in Māori, encouraging children to come in. Sometimes there is a mat-time with singing and/or listening to a story, and sometimes children go straight to handwashing. Older children sit at tables, and younger children are placed in high chairs. After a chanted karakia (grace in Māori) children share pieces of fruit and toast, and have a drink. Once most children are finished they are free to put their cups on the trolley and leave, and in fine weather many are eager to return outside as soon as the rostered teacher is available.

(Section 7.2.1 contains a detailed description of mealtimes.)

After morning tea, the range of activities typically alters and expands. Inside, dough may be replaced with clay, a collage activity set up, a nook created with cushions around the musical instruments, a tape (for example, Bad Jelly the Witch) may be played. Outside changes tend to be more conspicuous. New spaces may be created; one day the corner of the deck is set up like an office with desks, computer keyboard and telephones; on other days cushions, rugs, and puzzles, books or musical instruments are placed there. Changes in the wider playground included: a tent pitched on top of a large tyre, the house-frame covered with black paper, a 'car wash' area created, a bike-riding course developed with cones and ramps. Older children sometimes create their own spaces. Grace and Maxine took possession of the canvas tunnel by shifting resources in there; older boys 
barricaded the high platform of the fort. Additional activities or resources are introduced; easel painting is often set up, water play (in one or two troughs) may be provided, perhaps carpentry or chalk drawing on the concrete. When novel resources are introduced - the large yellow truck, the digger, the defunct switchboard - the high level of interest they engender requires teachers to supervise closely.

Indoors children come and go, playing with Duplo and the railway on the mat, dressing up, doing a puzzle, using dough or art materials. This can be an opportunity for solitary concentration:

Mooloo sits alone at the collage table, with a white piece of paper on which she has carefully sellotaped several pictures ... and is writing [her name] with decorations of curls. She decorates every available space using red and pink and orange and yellow. Shows it to me, and then to a teacher who comes to the table — and then puts it into her locker. [8/8/05]

It can be a chance to spend relaxed time with a peer or teacher. When Fleur and Mulan go into the locker room, a teacher follows:
Fleur asks: “Where's dummy?" [She brings a dummy to use at sleeptime]
Teacher: “I don't know. Did you put it in your bag?"
Fleur: "Yeah"

Teacher: "Did you, there you go, it will be in your bag then" ...

[Fleur looks in her bag saying] "Where's dummy? Where's dummy?" Then she says [what sounds like] "Me got it".

Teacher: "You've got it"

Fleur: [sounds like] "Me got it"

Teacher: "Have you?"

Fleur: "No me got it, me got it"

Teacher: "Me got it" (thoughtfully) - then suddenly "Oh mummy's got it!"

Fleur: "In the car"

Teacher: "Oh mummy's got it in the car. Has she?

The peaceful atmosphere, with most children outside, is conducive to Fleur conveying her message, and the teacher comprehending it. 
Outside there are usually small groups in the sandpit, on the swings, using the challenge course, playing in and around the fort and riding bikes. Some play independently:

Angus was ... running on to the ramp from the side, landing on the single plank that runs crossways, and leaping off the far side.... he repeated the action again and again. [25/8/05]

A teacher pushing a succession of children on the swings watches activities while she talks with them. When the water trough is filled it attracts many children. Teachers circulate, responding to children's interests. When she notices Robert T. is engaged in pouring, the teacher provides him with a plastic jar and funnel. As the jar fills she directs his attention to the overflowing water:

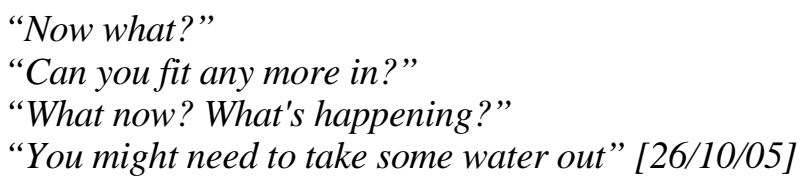

Watching his actions she comments: "When you put your hand in, water comes out".

Teachers sometimes take a leading role in facilitating activities. When the carwash tunnel was built (using a curved metal frame covered with blue cloth) a teacher stood at one end taking 'money' and giving 'change' before children rode through. Another day a teacher led an energetic game of lion hunting. There were two longer-term outdoor projects which occurred during the data-gathering period; the on-going maintenance of the garden and the construction of the house-frame.

For younger children, nappy changes are woven through the day, offering moments of contact and conversation with a teacher. As a teacher is changing Emjay she notices he is looking at a tortoise in the bathroom mural; she shows him the deaf sign for 'tortoise' and together they look for other tortoises on the walls.

A call to tidy precedes the pre-lunch mat-time. Teachers take turns in leading mattimes, which typically include a variety of stories, songs and activities. (Section 7.2.2 contains a discussion of mat-times.) Four days a week children are served a cooked lunch; on the fifth children are able to serve themselves from a smorgasbord. On the designated school-lunch day, 4-year-olds sit together eating their own packed lunches. (Section 7.2.1 contains a discussion of mealtimes.) As children finish, those scheduled to sleep are encouraged to strip to their 
underclothes before going to the sleeproom where they are settled by teachers. (Section 7.2.3 contains a discussion of sleeptime.)

Non-sleepers join a teacher for 'quiet time' in the quiet room. Very young children who have already slept play alongside in the Babysafe. The agenda for quiet time varies; a teacher might read stories, organise a group activity such as a memory game, lead a discussion or let children choose to do puzzles or look at books independently. These non-sleepers are then released into the main playroom to find a new range of resources set up-typically resources rarely seen at other times, e.g., a variety of construction sets, floor puzzles, face paints, painting with cottonbuds, etc. (Section 5.2.3 contains a more detailed discussion of this period.) As children wake, and teachers return from lunchbreaks, the doors to the outside are opened. When interest dwindles in the indoor activities they are packed away leaving the more familiar resources.

The remainder of the afternoon's activities are typically a relaxed reversal of the morning, punctuated by afternoon tea (sometimes preceded by a mat-time), and then by the gradual departure of children and teachers. The first child regularly leaves at 2.15 , and by $5.30 \mathrm{pm}$ only five or six children are left. As numbers dwindle, and areas are tidied, doors to the quiet room, the yellow room and the outdoors are shut. Teachers understand children's tiredness and often lead a peaceful activity_-singing along to the guitar, or reading a story with a puppet accessory. Occasionally a late-afternoon snack is served. The last child is picked up at $5.50 \mathrm{pm}$.

\subsubsection{Planning documentation for a typical six-week period}

Teachers worked to a six-week planning cycle, drawing on observed interests during the previous six weeks to develop a new collective focus. Simultaneously they also developed individual objectives for a group of children, working on a rotating cycle. The collective focus identified for the period August-midSeptember was:

For children to develop and take pleasure extending their interest and knowledge of the wider world focusing on relevant happenings and family events. [Prbbc21]

Teachers' ideas for how to sustain/extend this collective interest included: books on people at work, on diggers, trucks and trains; walks to the construction site and 
around the neighbourhood; letter boxes and posting letters; receiving visitors, e.g., police, dog control officer, school teacher, fire department; diggers and trucks in the sandpit and mulch; dramatic play—supermarket, restaurant, office, hairdresser, doctor, school.

During these six weeks teachers recognised an on-going interest in construction, took a group to the nearby construction site, purchased a truck and digger plus related books and posters, and used "correct names and lots of descriptive language" [Prbbc21]. Older children also went on a walk to the local school. An interest in transporting was noted, even among the youngest, both inside and outside.

Other 'spontaneous everyday experiences' and interests recorded by teachers over the period were:

Digger, new trucks, cones on mulch area

Trough with mulch, pulley, diggers, buckets, scoops

Guttering attached to fort, hard hats

Digging holes and transporting mulch

Mulch from fort down pipes - viscosity, force, direction, weight, volume

Transporting loads and passengers on green and red trucks

Delivery of new rubbish bin - group of children went to entrance way to watch bin being hoisted off truck

Water - transporting water between troughs, washing babies, tipping and pouring

Blowing bubbles

Car wash with bikes and trolleys

Ramps

Carpentry

Tree climbing

Playing musical instruments

Drumming boxes, tapes, drums

Dramatic play - animals from the zoo, dogs, dragons

Hairdressers and face paint

Baking pizza for fun lunch.

As these notes indicate, teachers were consistently recognising and responding to children's interests, and introduced many of the strategies they had earlier 
identified. They revisited these noted interests and experiences when they planned the collective focus for the succeeding six weeks:

For children to develop and take pleasure in extending their curiosity and knowledge of the centre environment and their wider world. [Progpl4]

Teachers also reviewed learning stories for a group of children, and set individual objectives. While learning stories for these younger children (who were the focus group for the period) showed many of the interests listed above, the objectives set tended to reflect teachers' commitment to ensuring children's individual and collective feelings of well-being and belonging. Anakin (20 months), Fleur (2 years 1 month) and Jordan (2 years 4 months) shared the same objective: "to develop concepts of self in the context of the centre and at home" using a range of strategies such as "routine times, looking in mirror, sitting at table, etc; language such as you, I, yours, self, etc; links with home, photos, language, names, etc; turn taking, -- ‘s turn, etc" [Prbbc21]. For Sunshine (11 months), who appeared nervous without an adult nearby, the objective was for her to develop her gross motor skills (and confidence) while in close proximity with others. The objectives for both Thomas (1 year 11 months) and Amanda (3 years) focused on their expression of caring and considerate attitudes to others. However, for Sina (3 years 10 months), the oldest child in this group, the objective was to build on her interest in caring for dolls/babies by exploring other care routines such as bathing, feeding. This emphasis on social dimensions in planning for individual children will be revisited in 8.3.3.

This section has explained the centre layout and described a typical day, and a sample of six-week planning in order to give an indication of the current scope of planned curriculum which constitutes the content of that central circle in Figure 1. For the remainder of the chapter the focus is on how the physical environment influenced the boundaries of curriculum.

\subsection{The centre's physical environment}

\subsubsection{Implications of the adult-child demarcation embedded in the centre's location}

The centre is physically isolated, sited at the back of a workplace complex, adjoining the back boundary fence. Here a rectangle has been fenced off for the centre building and playground, with a high concrete wall concealing adjoining residences. Along the other two sides of the playground high wire-mesh fencing 
looks onto a perspective of grass and hills in one direction and across the driveway and parking lot, towards the workplace complex in the other.

On one level, this physical separation from adjoining residences and the workplace could be interpreted as reflecting the value given to young children and their education. It was a peaceful oasis with only the sound of an occasional car, or a delivery truck to the nearby construction site to interrupt the medley of voices and activity. On another level, the physical separation of the building could be seen, as Singer (1996) and Brennan (2005) point out, to reflect the separation of children from the ongoing activity of the community, from the world of adults, and more fundamentally as positioning children as 'other' than adult (Cannella, 1997; Singer, 1996). The impressions of isolation were reinforced by the centre's high fences and tall gate.

Children showed they might not value the isolation as much as adults. They often interrupted their activities for a plane or passing car; when a large truck brought equipment for the building site, almost every child lined up to watch:

I noticed Emjay standing by the fence, looking out. The very tall crane over at the building site was moving, and he was keen to tell me about this. "Doo da" he said, and pointed through the fence towards it. He 'talked' about it with me, and I responded to his interest and talked about the crane, and how it was moving. [19/8/05]

The proximity of the building where some parents were employed further underlined the separation of children from their parents' working lives. When Maxine was talking about her photographs of favourite places in the centre, she added "I do like the studio where my mum works". However, visiting the workplace complex was not an event that occurred during the research period; indeed, trips out of the centre were rare. Detail on the centre's walk chart suggested some reasons for this:

\footnotetext{
Ratios: 1 adult to 3 children mixed ages (Maximum 2 children under 2 years) 1 adult to 8 children 2.6 - 5 years

Trained teacher

First aid kit [Progpl4]
}

These first impressions, linked to the centre's location, reaffirmed what others had written about the physical isolation of centres. The next paragraphs explain how, unexpectedly, the theme of the adult-child demarcation, and the associated exclusion of children from the adult world re-surfaced in the centre's physical environment. 


\subsubsection{Implications of adult-child demarcation embedded in the centre's architecture}

Conversations with children, focused on the folder of photographs of places in the centre (Research strategy 1), showed which areas were familiar to them, and suggested what might be important to them about different spaces, as well as helping me identify the names they used. It was children's comments here that first drew my attention to the strong demarcations about who could go where in the centre and provided insight into the limits on their movement. There were some places, such as the Babysafe, the deck and the children's bathroom that everyone knew and named. There were other areas that all the children recognised but did not have a shared label for. The small room, off the main playroom that was painted yellow, and where the dress-ups and dolls were kept, was labelled by one child as the "yellow room" or "dress-up room", another added "yellow group" (reflecting occasions when small group mat-times were held there I suspect). Jeff named it the "girl room" but after a moment's thought amended this to "the girl room and boy room and the pretend babies". Children pointed to show they recognised another of the adjoining rooms that was regularly used for play; one child called it the "quiet room", which reflected its use as the area where nonsleeping children gathered after lunch.

Children's responses showed there were some areas of the centre with which they were much less familiar. Most of the older children recognised and named the kitchen which was separated from the main playroom by a mesh door but not all the younger children knew it. Only some children recognised the door in the foyer that led to the office; one child labelled it as "where you go home", another suggested it was the door to the storeroom. Yet this was a door they passed as they came and went, and through its glass panel they could see the manager at her desk as they moved between the locker room and bathroom. Only one child recognised and named the staffroom. As an adult moving freely through these spaces, I had overlooked how strong the demarcations were for children, and had not anticipated how unknown some areas were to them. The strongest analogy was with my own memories of the school staffroom on those rare occasions when I glimpsed inside it. Similarly I had overlooked how little children knew of the adult tasks that took place in those less familiar places. Children did not identify the laundry with the task of washing clothes, a regular task but one with which they had no contact. Instead it was named as "where they make the dough", 
"where the buckets are". Drinking coffee and eating lunch were not talked of in reference to the staffroom.

Initially I defined places as 'adult spaces' and 'child spaces' but realised it should be 'adult spaces' and 'communal spaces' (see Figure 5). Teachers could freely go into any communal spaces but not vice versa. There were only two tiny places in the centre that might be defined as children's spaces, places where teachers did not go; these were inside their locker spaces and into the child-sized kitchen cupboard, although teachers rarely went under the lower parts of the fort or through the fort's wooden tunnel.

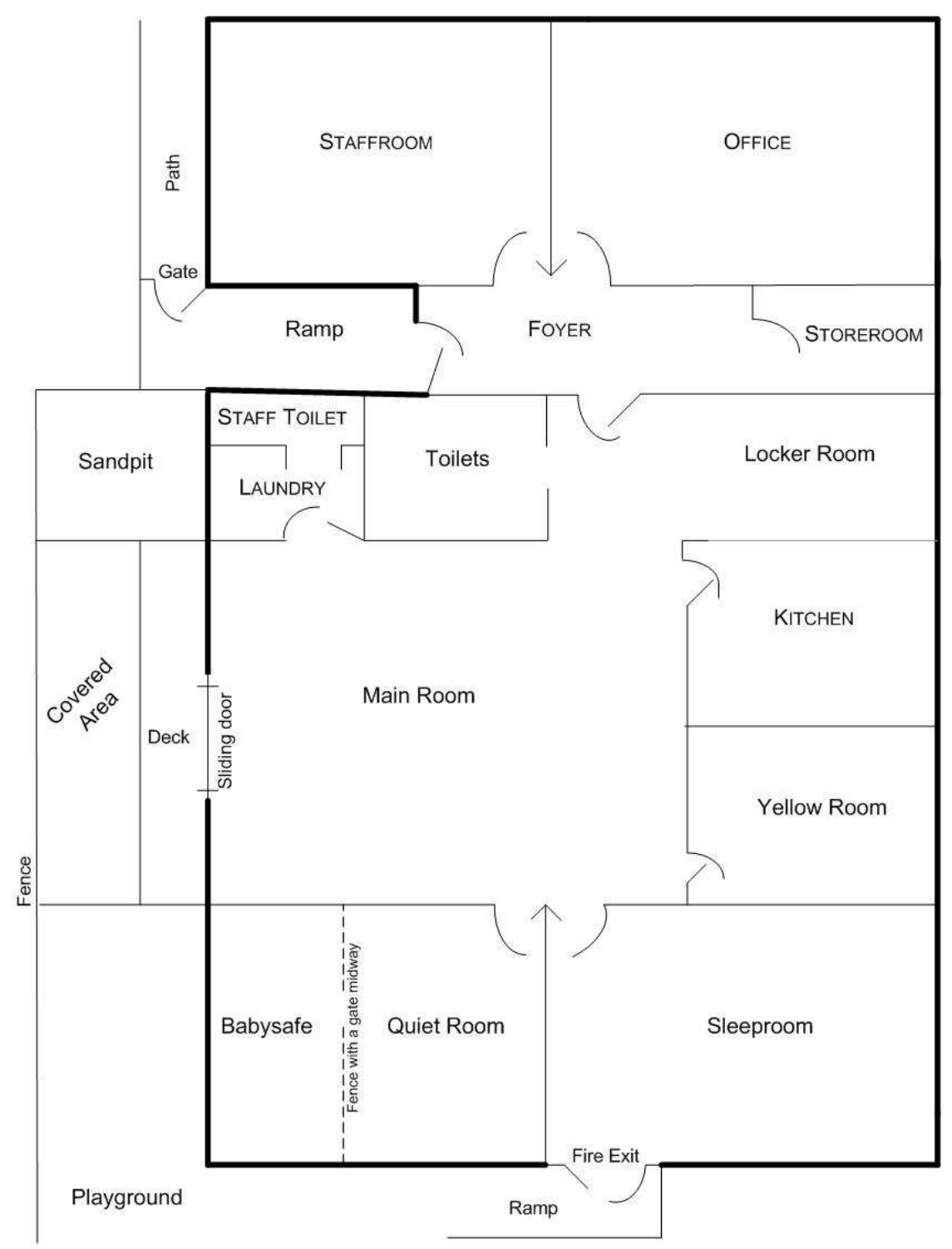

Figure 5: Adult spaces and communal spaces in the centre (Capital letters indicate areas defined as adult spaces) 
Once children had alerted me to the distinction between the spaces, I recognised that the doors reinforced this distinction. Adult spaces had full-height doors. Some of these doors still allowed children to see through; children could look through the gauze of the kitchen door, and taller or carried children could see through the window in the office door, but the roster sellotaped to the laundry-door window restricted visibility. Handles on all three doors were at adult-height reiterating these were adult spaces. Other doors implied a sharper demarcation. The door into the sleeproom had a smaller adult-height window and was latched with a high hook. (While the sleeproom was theoretically a communal space, it was not a space children could freely enter. Evie was reprimanded when she climbed on a chair and undid the latch.) There were no windows in the doors to the staffroom or storeroom, and their handles were at adult height.

Communal spaces, in contrast, either had no door or had a sectioned door, with two independent parts. The two smaller playrooms and the doorway between the locker room and foyer all had these sectioned doors; the lower door was used during the day, while the higher section was typically latched back. Reflecting on the doors, I wrote:

The [lower] door ... is at the same height as the tray on the high chairs. The smaller children would not be able to see over it. It has a handle on the outside to push it with-but it also has a bolt on the inside that you need to lean over and undo when it is shut. The open door means the area is inviting, especially because as an older child I can see over the door. The bolt says this is an adult space. I can ask for equipment-but that does not give me access to this quiet retreat space. [20/8/05]

There was no door in the doorway to the bathroom or between the main room and the locker room.

There were innumerable features in the design and décor of the communal areas that conveyed to children these were places designed for them. The child-size fittings and furniture, the low doorways and windows, the low handbasin and toilets in the bathroom area, and the low lockers all conveyed this message. The youngest children were catered for with the Babysafe, and with cots, highchairs, the changing table and the potties. The valuing of children was reflected in the effort that had gone into, and continued to go into, making the play areas vibrant and colourful. An underwater mural across the bathroom walls and ceiling, and large New Zealand bush murals in the main playroom and across the playground's 
concrete wall contributed to this impression. Only the sleeproom, with drawn curtains, dimmed light, and little on the walls, was an exception.

Adult spaces looked different. They were decorated in muted colours, the surfaces were darker, and there was little on the walls; the contrast with the vibrancy of the communal play areas was striking. There was a tidy, functional air about them; these were places of work. Only the staffroom with its sofa and easy chairs, its crowded noticeboards and coffee-making corner had a slightly more relaxed feel.

In practice this demarcation between adult and communal spaces was occasionally breached. A child might visit the office as they came and went with a parent, children went into the kitchen and were then gently shepherded out. The most striking incursion of a child into an adult-only space occurred when Aidy disappeared into the adult toilet while his mother was in the centre, preparing to take him for an appointment. The astonished laughs among the teachers suggested the perceived audacity of his action but, probably because his mother was present, no comment was made when he re-emerged. This demarcation between adult and communal spaces both embodied and embedded the categorisation of children and adults as inherently different. It seemed that even within the centre children were "sited, insulated and distanced" (James et al., 1998, p.37) from the world of adults. By and large the architectural demarcation between adult-only and communal spaces was reflected in practices, and so provided moment by moment reconfirmation of the underlying categories of adult and child, and reinforced the distancing of children from the world of adults even within the centre. This was an unanticipated insight, although Dahlberg and Moss's (2005) vision of children and adults "being and thinking beside each other" (p.28) implied other possibilities.

\subsubsection{Implications of the adult-child demarcation embedded in the centre's} resources

A focus on physical resources reinforced and extended this theme of adult-child demarcation; it was reflected in the range of resources and expectations around children's use of them. The description of the resources for children below shows that the range largely replicated what might be found in any well-resourced mainstream New Zealand centre. 


\section{Resources for children indoors}

\section{Main playroom}

The resources in the main playroom were accessible to children except during mattimes, mealtimes, and the quiet period after lunch.

\section{Around the mat:}

Low shelves holding containers of Duplo, the wooden railway set and wooden unit blocks, musical instruments

Selection of 10 to 15 books, and often a few puzzles kept on top of the shelves

(These shelves were sometimes covered with a fabric throw-over for mat-times.)

\section{Collage area}

Large circular table

Low shelves containing collage materials, Sellotape, scissors, paper and felt pens Container of cardboard boxes, cylinders, etc.

\section{Playdough area}

Large circular table

Child-sized kitchen units (sink, stove, dishwasher, microwave, cupboards) Playdough and kitchen/cooking equipment

\section{Nature table area}

Small nature table and fish tank plus natural resources

Large circular table, often with books and/or puzzles

\section{Quiet room}

Storage units containing a much wider range of books and puzzles, plus soft toys.

Children's portfolios were kept on top of one unit

Large cushions

The fenced-off Babysafe (an enclosed area for the youngest children)

Cushions

Low shelves containing a selection of toys such as a posting box, soft toys, rattles, plastic blocks and an activity centre 


\section{Yellow room}

Open wardrobe containing dress-up clothes and shoes

Coat-stand holding bags and hats

Dolls' beds, dolls, dolls' clothes, small pushchairs and highchair

Child-size bed

Computer keyboards and cell phones

\section{Resources for children outdoors}

\section{Covered area around the deck}

Sandpit plus wide range of equipment

Deck

Often a corner was set up with cushions and a rug, and perhaps musical instruments, puzzles, books or a tape to listen to

\section{Concrete-surfaced area}

Activities such as painting, waterplay sometimes set up, more rarely carpentry, clay, fingerpaint

\section{Playground}

\section{Bark chip surfaced area}

Swings

Two-child stationary car

Fort - two high platforms accessed by stairs, and two vertical ladders. The two platforms were connected by a wooden tunnel. One platform led to a slide, a zoom slide was occasionally attached to the other. An enclosed space under one of the platforms had an open window and doorway.

\section{Safety matting surfaced area}

Challenge course equipment, bikes and ride-ons, and balls were almost always available 
The presence of books with a Māori theme, and/or using Māori language, tapes of Māori music, and puzzles and posters displaying Māori images reflected the commitment to biculturalism inherent in Te whäriki, and now required in every New Zealand centre. In many other ways the resources provided would be similar to those teachers might remember from their own New Zealand childhoods. While it is not within the scope of this work to trace the evolution of traditional early childhood equipment, it is thought-provoking to consider how little these resources have changed in the last 50 years (Somerset, 1967), how distant their origins are from New Zealand in the 21st century (Roopnarine \& Johnson, 2005), and how rarely these traditions of provisioning are questioned (Cullen, 2003a; Hill, 2001).

The centre was perhaps atypical in the quality and quantity of the resources provided for children. There was an unexpectedly vast array of equipment in the indoor storeroom. In comparison, for most of the day the range of resources in the centre seemed relatively sparse. The profusion of resources in centres has often attracted comment (Anning, 1991; King, 1978) but my inventories showed there were usually only around 40 books available and between 10 and 15 puzzles, although these were regularly rotated. A teacher picking out a new selection of puzzles listed factors that were influencing her:

Some simple ... some old favourites, some of the recent ones that have been very popular... some insect and animal ones, some with a moral message [18/10/05]

Teachers worked hard to maintain the environment, and at staff meetings were reminded about keeping areas tidy. Consequently, unlike classrooms King (1978) describes where every surface was covered, this environment had a sense of wellmaintained order. This impression was enhanced by the fact that broken equipment was removed until it could be mended or replaced. Children were therefore accustomed to working in a very attractive and orderly environment with a range of well-maintained resources.

Teachers brought in additional resources, many of which were permanent additions. Some were free resources, such as natural materials, and clothes converted into dress-ups, but many were paid for personally, and very generously, by one of the teachers. Among the bought resources that appeared were: books, additions to the railway set, a timer, animal dress-ups, a bike, sit-on diggers, rattles and wooden puzzles. I was often aware from attending staff meetings that teachers were responding to children's identified interests with equipment they introduced. 
There was a clear distinction between equipment for adults and for children. Even in the communal areas there were spaces and equipment that were defined as adult-only. In the sleeproom there was a high shelf for the CD player, and in the main room there were shelves used for storing finger puppets, $\mathrm{CD} /$ tape player, CDs and tapes, and the teachers' camera and notebooks. Children's portfolios were stored out of reach in the quiet room, but they could ask to look at them. Most children could not reach their family's fabric 'wall-pocket' in the locker room, although they enjoyed looking at the family photographs that adorned them. Children typically did not have access to resources defined (by teachers) as teachers'. They did not use the CD player, they had no access to the computer or photocopier in the office, or to the centre camera. While such restrictions are professionally and practically reasonable, the fact that teachers have since shifted the portfolios so children can reach them shows that such decisions are not immutable.

The distinction between adults' and children's resources was occasionally breached. It was breached when teachers brought in personal possessions; one teacher brought two books about horses and a book of earth-moving machines to share with particular children, another shared her photographs of a family trip to Disneyland during quiet time. It was breached when Mooloo was given the teachers' felt pens to make a birthday card and when children were given kitchen knives to make sandwiches for afternoon tea. It was breached when teachers used children's resources for their own purposes - a teacher used pastels to draw a landscape while a group clustered around her watching and commenting; a teacher moulded a car shape in playdough as a trial for a birthday cake she was to make. The sense of adult-child community these moments engendered was striking, as it was in those episodes when children and teachers participated with a shared agenda, using children's resources. Such a blurring occurred when two teachers and a group of children worked together drawing and colouring in pictures of sea creatures which they stuck to a blue cellophane 'sea' on the window. It occurred when a group of girls and a teacher planned a 'barbecue'; the girls loaded a tray up with plastic crockery while the teacher wrote down their suggestions for food they would need:

\section{"Right" she says "is that everything? I will give the list to [the centre manager] and let her know". [7/10/05]}

I noted: The line between pretend and reality is satisfyingly blurry. 
My introduction of a camera for children to use on photo tours also breached the demarcation, and children's reactions suggested the significance of this. My abiding impression was that using the camera took priority over thoughtful selection of places for almost every child; having access to a digital camera was not only a novelty, but it gave children access to a tool defined as 'adult' (Nimmo, 2008) which was rare, perhaps not only in this setting. When I told Aidy I would hold the camera while he had a swing he replied "My mum said I can swing with the camera" but acknowledged he did not use hers. The opportunity to be in charge of the camera was a significant attraction for 4-year-old Rex who became adept at using a range of camera functions. The hammers, small handsaws, and pliers available when carpentry equipment was out, and the battery-powered drill which was introduced during the building of the child-size house-frame, were other examples of adult equipment children used. The drill was the resource that most significantly challenged assumed parameters of appropriate/inappropriate equipment for children in a New Zealand centre setting. However, it was a resource used by a teacher for a single project.

Teachers' care in preparing the environment for children was most obvious in the period of indoor play which followed the post-lunch quiet period. At times it seemed teachers vied in their creativity in setting up for this period:
After quiet time when we come out [the teacher] has set up the room with two tents-a red one and a blue and white striped one on the mat, with dress-ups and the curvy mirror from the Babysafe beside the tents. There is also an array of necklaces set up on a bench-the area is more divided up than it usually is with a bench beside the mat, and another bench down by the glass doors with plastic woodwork tools-two drills, a sander, a level, a circular saw. ...The dough table has balls of green dough with sparkle in $i t$, and a basket of equipment in the centre which includes shells, as well as rollers. ...The drawing table has black pieces of paper, and coloured chalks in egg cartons set around at each place. [The teacher] does face painting, and she allows children to do their own if they want. [17/8/05]

Of this array, it was facepainting that attracted most children until the outside doors were re-opened; then those still waiting, and Sina and Evie who painted their own faces, stayed longer. While the environment looked enticing and unfamiliar, many aspects did not seem to engage the children. For example, I noted:

Most of the children out are the older children, and these [carpentry tools] seem to lack purpose. Children roam using them-Mooloo has a drill and bangs it up and down on her chalk drawing, Rex walks around the drawing table hitting it with the drill rhythmically. 
Next afternoon pebble tiles, plastic dinosaurs and spiders were provided on the dough table, and pink, white and mauve Lego blocks were available on the nature table. On the mat there was a plastic construction set, a box containing wooden triangles, and a plastic road map and Duplo cars. I noted there was a surge of activity particularly around the dinosaur table, the wooden triangles and the construction set, but then sword-making at the collage table became the prime focus. It was not clear who initiated this, although Rex's was the first sword I saw and it was clearly child-made. Soon children's enthusiasm for swords led a teacher to take over the bulk of the making. Once children moved away, the novel aspects of the environment were soon dismantled; the scheduled duties indicated that activities not being used were to be put away by $2 \mathrm{pm}$.

It seemed there were inherent tensions for teachers here. There was a potential tension between the teacher supervising quiet time and the teacher setting up, just as there was sometimes a tension between the teacher setting up outside, and the teachers with children inside. This was also a period when teachers might cater for the older children, but the youngest children's sleep patterns meant they were often present. Teachers also considered providing indoor activities that some of the boys would find absorbing was a challenge, given their orientation to physical outdoor play. Children missing the companionship of their sleeping peers might contribute to these difficulties:

Rex seemed to be at a loose end ... he wanted to know what I was doing. Also, he was reading the sleep chart-and James, Aidy, Alex and Angus were all sleeping. [30/9/05]

The underlying constraints for teachers were that children needed to be kept inside and relatively quiet until there were sufficient teachers available to cover supervision both inside and outside - the provision of novel equipment imaginatively presented was an understandable response. However, reflecting on the novelty of provision, I wondered whether "all this setting up leads to an environment of entertainment rather than engagement" particularly in light of the short time for which it was available. Such a wealth of provision might indicate children were positioned as players (Cannella \& Viruru, 2004), a notion perhaps underlined by the plastic carpentry resources introduced here, in contrast to the real drill that was later used with children. This notion will be returned to in Chapter 6. 


\subsubsection{Children's activities: Further evidence of the adult-child demarcation}

Other implications for children's curriculum experience also emerged from the analysis of the adult-child demarcation. The environment encouraged busyness but there were also implications regarding the length and quality of children's resource-based activities which indicated underpinning assumptions about the quality of children's activity.

The variety and quantity of resources, the fact tables were regularly set up with activities, and through the day new resources were produced indicated the value placed on children being busy. The environment suggested children would find something to interest and absorb them, and children's level of activity and engagement indicated they did indeed find the environment stimulating. Evie shared the photograph of the giant robot she had made; Mulan and Fleur, painting at the easel, squealed with delight when they looked at each other; Sunshine was absorbed in rolling the drum (that contained a construction set); Aidy was "delirious with joy, dancing, singing, clapping, stamping" when the CD of The Lion King was played. Sometimes it was a single activity/event that attracted children; one morning the playground seemed deserted because children had gathered for an indoor baking activity. More often children were engaged in a range of activities. The wide range of positive responses children gave in identifying activities they liked/disliked reinforced the impression of their enjoyment-collectively children made 169 positive selections and only 14 negative. In comparison to what might be a typical New Zealand home setting, the range of resources, the relative freedom in using these, plus teachers' tolerance for mess, noise and exuberance potentially made this a very attractive environment for children.

However, aspects of the temporal and physical organisation were not conducive to longer-term resource-based activities. Mealtime routines meant tables needed to be cleared three times a day; keeping the environment attractive meant indoor and outdoor spaces were regularly tidied. Occasionally children's constructions were protected, but there were limited spaces to store ongoing projects. When Mooloo and a companion were working on a large block construction:

... cones were placed around it to keep it safe through morning tea. [12/8/05] 
Sometimes a window ledge or the top of the block unit were used for temporary storage:

Thomas is asked to go and wash his hands, but seems to be finding it hard to leave the little Thomas engines on the mat. The teacher tells him that she will put his linked engines up high so they will be safe until after morning tea. [19/10/05]

Only the racks for drying paintings and collage provided regular storage. I noted one afternoon that a few collages were the only tangible evidence of what had happened in the centre during the morning. There were only two longer-term resource-based projects for children. One was the two small garden plots, one for flowers and one for vegetables, which had already been planted, but which required on-going weeding and watering, and eventually the harvesting of the vegetables. The other was the construction of the house-frame which was facilitated by one teacher and lasted over several weeks. In contrast teachers had ongoing tasks that were often worked on over several days, and stored, in the adult-only areaspreparing displays documenting children's learning, posters for a parent-education display, children's portfolios—which highlighted assumptions embedded within the demarcation between adults and children.

Others have drawn attention to children's dwindling access to, and participation in, the lives of adults (Brennan, 2005; Singer, 1996; Strandell, 2000) and this has been linked to their marginalisation from 'real' tasks (Nimmo, 2008). Children's participation in a few regular tasks associated with routines will be described in Chapter 7. Other resource-based tasks that were real in the sense of contributing to the life of the community were infrequent: the baking of a birthday cake for the centre manager, making a birthday card and birthday hat, spreading bread for afternoon tea, helping with feeding younger children, harvesting vegetables, and constructing the house-frame. However, one needs to be wary in defining what children may regard as 'real' tasks. Perhaps the distinction between expendable and non-expendable materials is relevant here; Mooloo's careful collage work which she put in her locker to take home may have felt more like a real task than Jeff's sandcastle which was inevitably destroyed. Older children may have considered the occasional 'writing' sessions, during which they practised letter formation, to be a real task. Teachers may have been discouraged by children's frequent reluctance to engage in the only regular 'real' task-tidying (to be described in section 7.1.1). There were, however, some occasions when children appeared to enjoy demonstrating their tidying prowess: 
The children who had played in the dramatic room ... were asked to come and help tidy it. ... Maxine volunteered to make the bed, and made an excellent job of it, tucking under the sheet neatly at the corners, and putting throw cushions on the top. [10/8/05]

A teacher (who was present during part of a parent interview), described Evie's competence with the drill to her mother and suggested the significance of that experience for Evie:

"For her the ideal is having that special time with a teacher to do something that is meaningful ... She doesn't want to just bang nails in wood, she wants to make something that has meaning." [18/10/05]

Other observations provided corroboration for this interpretation, but also indicated the difficulties that might arise if 'adult' tasks were delegated. When a teacher was opening the shed:

Evie is very keen to have the keys and to undo the padlocks holding the door on either side.

She ignores offers of help, perseveres and unlocks both with the teacher's encouragement.

The teacher asks for the keys so she can put them in her pocket. Evie says she will put them in her own pocket. The teacher explains they need them when they come to lock up.

Teacher: "Go inside and give them to [a teacher] or give them to meyou've got a choice". [18/10/05]

Delegated 'adult' tasks could also require intense teacher input.

Robert T. needed considerable support when he made his own sandwich for afternoon tea. A teacher saw he was licking the knife and warned him:

"Robert, if you put your knife in your mouth, you could cut your tongue. You need to leave it on the table".

She goes to get him a clean knife, and he puts his knife back in his mouth within a second of her leaving. [6/10/05]

He continued to dip his knife in the margarine and lick it, and his knife was replaced several times. Eventually, the teacher guided his hand through the motion of spreading the margarine, encouraged him to add lettuce, and then fold the bread over so he could eat it. Such moments underline the challenge facing teachers as they balance the desirability of offering children such experiences, with the reality of teacher time and energy that may be absorbed in supporting a few children. 
Two of the six parents who were interviewed volunteered that assisting an adult in a real task was a significant theme of their child's home activities.

[Aidy] likes to help $G$ [her partner] ... for instance last night he helped mending the garage door. He gets very upset if he is in bed and there is some mending or fixing going on. [18/10/05]

This brings to mind the advice Singer's young friend offered her (Singer, 1996): "When looking after children you should do something that you yourself enjoy or value. Then children come to you by themselves to watch, to ask something or to help" (p.34). This question of who did real tasks, and when and where they were done will be revisited in Chapter 6.

Considering children's interests and what was/was not present in the centre offers another aligned perspective on the matter. Children's interest in people, and particularly in others' families, was constantly sustained as family members came and went. When a teacher's two school-age sons visited the centre, I noted:

It is interesting how the others appear where they are. When they sit at the dough table, Thomas and Alex and Aidy appear there. [27/9/05]

Children's interest in nurturing was supported through the provision of dolls and associated resources, but it was also supported by the presence of very young children in the centre. Amanda hugged Caitlin (1 year 7 months); James encouraged Macauley (1 year 1 month) to ride on a truck like his. A visit of police dogs fed children's ongoing interest in animals, and this was supplemented with animal-themed books, animal costumes and displayed photographs of wild animals.

Not all interests were so consistently supported. A teacher created a horse scrapbook for Cassidy, including his drawings and photographs and stories of her own horse. Sharing it showed how familiar he was with the content; he recited the caption it "ate too much spring grass and got sick". But when Cassidy shared his portfolio he explained "There's only one horse" and showed me a drawing which he said his mother had done. Cassidy usually had access to plastic horses in the centre, but comments from teachers suggested they felt a tension between sustaining Cassidy's interest in horses, and extending his interests into other areas.

Maxine's selection of favourite activities began "I like drawing, cooking, playing, Duplo, reading the books" and when I later asked what she had learnt to do while 
she was at the centre, she volunteered "cooking". Maxine and Grace repeatedly 'baked' with playdough, and typically appeared absorbed in their domesticthemed dramatic play. Towards the end of Grace's photo tour she led Maxine and me to the sandpit, where she began:

... "making a cake", picked out a roasting pan from the sandpit toys, and started to put handfuls of sand in it. ... Maxine became absorbed in gathering up dried sand from all around the perimeter and sprinkling it onto Grace's cake. [4/8/05]

Opportunities for real cooking appeared rare; I observed two episodes (cakebaking and preparation of afternoon tea), and noted references to pizza making and Christmas baking in planning documentation for the data-generation period.

Rex's mother described him as passionate about electronic gadgets:

He is into everything electronic. There are 3 remotes in the house-for Saturn, TV, DVD. When the grandparents are there and want to watch the news, they ask Rex and he sets it up for them.

He loves using the digital camera, the phone that takes photos. He doesn't play around with her phone -it won't take photos. [2/11/05]

But in the centre he only had regular access to detached keyboards, old cellphones and occasional access to my digital camera. However, conversations with parents, including Rex's mother, indicated families supported the centre policy of not providing children with computer access. Evie's mother explained:

I don't want them to see the computer as an activity. We don't want them to lose their imagination. [18/10/05]

Fleer (2003a) describes the boundary of the learning environments in Reggio Emilia as extending out into the community; here the boundaries of the learning environment seemed to be closely aligned to the centre's physical boundaries, and philosophically aligned to traditional tenets of early childhood education. The pressures and parameters within which the teachers worked made it very difficult for this to be otherwise. As Brennan (2005) concluded in another New Zealand centre setting:

Socio-cultural approaches promote cultural, social and historical embeddedness yet in applying such approaches to early childhood practice and research we separate rather than embed children and their teachers, at the personal, community, and institutional levels (Wenger, 1998). My concern is that we are setting teachers an impossible task when asking them to enculturate children into society using a theoretical base that promotes community connectedness and involvement, yet the act of group care itself separates teachers from their own communities. (Brennan, 2005, p.213) 
The findings in this setting reiterate her concerns. It seemed that the adult-child demarcation seen here was a reflection of a much more widely accepted regime of truth which acted constantly to deflect teachers from a conception of children as competent partners and back to a developmental 'gaze' which positioned children as other. Further implications of the adult-child demarcation which arose in considering teachers' and children's roles will be discussed in Chapter 6 .

\subsection{Issues of power and control}

Reviewing the literature on the messages implicit in the environment and resources (Chapter 3, section 3.1) highlighted the adult-child demarcation, and also showed that teacher control, wielded often unwittingly, is a recurrent theme, particularly within the writing of reconceptualist scholars. Walkerdine (1992) suggests that such domination is concealed within the role of the benevolent and maternal teacher. This notion of concealment is reiterated by Cannella and Viruru (2004) who argue that the power and control adults hold, which enables them to exert both physical and intellectual domination over children, is typically exercised in covert ways. Within the New Zealand context, Boyce (2001) has reflected as a teacher on how power is exercised in relation to physical space, routines, and access and use of resources. Unquestioning acceptance of assumptions about the balance of power between children and adults suggests it is a further dimension of the regime of truth which defines adults and children as inherently different. Understanding the ways in which centre teachers exercised control in relation to the physical environment and children resisted that control, extended my understanding of how the adult-child demarcation was enacted in this setting.

\subsubsection{The centre's architecture: Implications of power/control}

There were implications in the architecture which echoed critical pedagogy's focus on the role of power in educational contexts and the ways in which such power is often concealed behind apparently benign educational discourses. More particularly, attending to the architecture drew my attention back to Cannella and Viruru's (2002) assertions that the freedom of movement implied by open layouts is illusory, that adults control children's choices and that children are governed through the very pretence of freedom. 
While children almost invariably appeared delighted to arrive at the centre, greeted teachers warmly, and were quickly absorbed into ongoing activities, the lack of child-appeal in the centre's external approach (in contrast to MacDonalds, for example) underlined the fact that children were not expected to be part of decisions about coming to the centre. The high fences, inaccessible 'child-proof' gate and the high front-door knob all indicated this was not a place where children could freely come and go. Observations and children's comments provided some confirmation for these perceptions. Children stood and waited for adult assistance beside both the gate and front door, indicating they did not believe they could open either, and on the very rare occasions when a child was unhappy, leaving was not an attempted option. When Angus was unhappy about having to sleep, he squeezed into his locker, one of the few child-only spaces in the centre.

Conversations suggested children saw mothers as controlling decisions about attendance. While children seemed to find it difficult to answer the question of why they came to the centre, three of the four children who did respond made reference to mothers. Mooloo told me "My mother decides what school I go to". Observations provided some further confirmation. Rex regularly negotiated with his mother about whether he had to stay at the centre, and when Mele (aged 2) was unhappy and wanted to leave, it was her mother she wanted.

Within the building, open doorways and low doors suggested children could move freely through communal areas. Teachers regularly asked "What would you like to do?", "Where do you want to go now?" which underlined this impression. However, beneath this apparent freedom there were considerable controls. While there were restrictions on teachers' movement dictated by the roster and regulatory requirements, the relative freedom with which teachers moved through the centre, together with the fact they controlled children's access to some communal spaces, conveyed messages about children's and teachers' relative levels of power.

Children understood teachers defined the shifting parameters of their freedom, and usually accepted these, albeit grudgingly at times. There were times of the day, such as early morning, mat-time, mealtimes, and the post-lunch quiet time, when children were usually required to be inside. 
Two or three times boys went and tried to open, or opened the sliding doors. ... Eventually a teacher said "We are going to need an outside teacher I think" and she went out and unrolled and secured the end of the deck tarpaulin [it was raining] before she let them out. [28/7/05]

One afternoon two of the 1-year-olds expressed their desire to be outside:

When [the teacher] went outside, Sunshine and Macauley were over at the door in a trice, and when she came back in, they were both still waiting there. Macauley shouted his annoyance and when the door was finally opened ... he was out like a shot, only to be picked up by a teacher saying "It's ok, you can go out" but putting his trousers on-he had been wandering around in nappies. [29/9/05]

Teachers typically defined rain as preventing children from going outside, as is normal practice in New Zealand settings, although here they were still able to use the outside covered area. However, the example of Norwegian forest kindergartens where children are outside, winter and summer, regardless of weather (Sandester, 2006) shows how culturally defined such judgments are.

Teachers also controlled which indoor communal spaces children could access during the day. Older children were not supposed to enter the Babysafe, and were encouraged to leave if they were found there. Younger children were occasionally restricted to the Babysafe:

A teacher brought Macauley through and put him in the Babysafe. He was vocal but accepted it-I assume he had finished his morning tea by then. Eventually a teacher opened the gate and said "Now you can go wherever you want". The other children were starting to wander and the doors were opened soon after. [18/10/05]

Interestingly, given it was an area of restricted access, the Babysafe was the most frequent positive choice in children's selection of photographs of liked/disliked activities/events/areas.

As an adult, I understood why teachers restricted children's access to the hard-tosupervise locker room and bathroom and regularly reminded children: "You need to come back into the play room". The bathroom could be the site for unplanned water play:

Fleur and Mulan [both just two] are found playing in the water in the toilet and they are supervised in having their hands thoroughly washed and then sent out. They move to the lockers and Fleur says "My bag here". The teacher tells them "We don't play in the locker room" and Mulan takes Fleur's hand and they move through. The teacher says "Good listening girls". [15/8/05]

It could be the site for peaceful companionship but also for unobserved conflict: 
James says "I need to go pooh". Alex says "I need to go pooh too" and they head off to the bathroom.

A few minutes later, I heard James say to Alex:

"You have to wash your hands, you're dirty", and then Alex is lying on the floor full length crying, and James finishes washing his hands, leaps over Alex and leaves. [9/8/05]

As the end of the day approached, children's access to communal spaces often diminished:

The doors to the outside are closed at $5 \mathrm{pm}$, and the quiet room and the yellow room are both shut off and tidied. [27/9/05]

Another day, at afternoon-tea-time I noted:

Choices are now very restricted - the main room, the bathroom, the locker room, and the deck-sandpit area (because it has started raining). [20/7/05]

There appeared to be unresolved tensions for teachers between maintaining the environment, smoothing transition times, and yet providing children with freedom of choice in play areas. Teachers were reminded at staff meetings both of the importance of an attractive tidy environment and that smaller play rooms should be kept open as much as possible. Sometimes the pressure of completing routines and maintaining the environment meant doors were shut once a room was tidy. This was particularly so towards the end of the day; the reality of such pressures is captured in this list of tasks teachers needed to fit in alongside their work with children:

Just a reminder about the duties that are required to be done before you leave each day...

3.00pm: Ensure washing is off the line. Mop bathroom floor, clean loos etc. Remove any items from top of breakfast bar

5pm: Fill kettle in staff room with water, wipe down bench top and table, replace cups if necessary.

5.30 pm: Empty dishwasher and refill if necessary. Any leftovers in fridge to be thrown away, etc.

6pm: Ensure windows and doors are locked (including fire exit door in sleeproom). Check baby monitor is turned off, etc. These are also to be in conjunction with the jobs as per roster duties.

Outside: At approximately 4pm start to pack away the equipment in the outside environment and lock the garage. Sandpit area swept clean or hosed down and on Fridays hose off completely, etc.

Inside: Collage trolley filled for next day - boxes, etc and paper restocked.

Blocks, etc picked up from under shelves and off floor, etc

Babies: Put bottom cloths on for washing, pack away beds and put out beds for washing, etc. [9/10/05] 
Children's resistance to teachers' rules forms part of Chapter 7, but it is relevant to note here that while some children appeared to accept the limits of accessible space, others resisted in a range of ways. Occasionally children unbolted a door:

At the end of morning tea I notice Sina lean over the yellow room door and pull the bolt so she can get in to the dress-ups-no one stops her. [9/8/05]

That taller children could open these catches, but rarely did so, suggests they knew and abided by the rule. Children were also reminded of who held the decision-making power:

Evie and [another child] are in the quiet room, and suddenly I hear a teacher call out "Shut the door. Who let Macauley out? Shut the door". A teacher goes in there, and Evie comes out of the Babysafe, the gate to the Babysafe is shut, and Macauley is back inside. [17/8/05]

Children's typical acceptance of these rules echoes Cannella and Viruru's (1997) statement that "Self-regulation is used as more effective than overt control, which may actually be more easily resisted" (p.134). Observing such features was partly what led me to write in my journal, within a few weeks of starting:

Power is a useful thought-how everyone seems to be exerting power over others. [15/8/05]

Other implications for children's curriculum experience flowed from teachers' control of spaces. Closing off areas made it more difficult for children to access withdrawal spaces such as the wooden tunnel or a cushion in the quiet room and it often reduced children's access to 'soft' environments. When the carpet and cushions of the quiet room, and the carpet and bedding of the yellow room were not available, the mat in the main room, and the carpeted floor and bench seat in the locker room might be the only accessible 'soft' areas. A further implication was that noise levels were sometimes high:

At $5.30 \mathrm{pm}$ I am sitting with Aidy on my knee reading to him, and find it difficult to make myself heard over the noise in the main room. [27/9/05]

While the characteristic sound was a cheerful medley of voices, Grebennikov (2006) has warned that centre noise levels can be harmful. The noise level could be seen to have implications for children's concentration and perhaps their ability to withdraw by filtering out contact, as Greenman (2007) describes, and it was yet another indication of the embedded assumptions about environments for children vis à vis adults. On several occasions, late in the afternoon, Fleur stood beside the block unit and rolled trains along the top; was this a strategy of withdrawal that allowed her uninterrupted solitary play? In contrast to children, teachers had 
regular opportunities to withdraw through the day. All the adult spaces were oases of quiet and calm, and the staffroom was a 'soft' retreat space with carpets and a comfortable lounge suite. Moreover, no staff member was regularly present from 8 am until $5.50 \mathrm{pm}$, but several children regularly attended for close to the full day. Mayall (2002) has noted "social assumptions allow for children's working conditions to be poor (by comparison with those legally enforced for adults)" (p.15). While physical conditions for both children and adults were of high quality in this setting, those differences in social assumptions Mayall refers to were reflected.

\subsubsection{The windows: Implications of power/control}

Just as doors were the cue to the distinction between communal and adult-only areas, the windows were the cue to messages about one particular aspect of teacher control, the supervision/surveillance of children. The large internal windows between playrooms, and overlooking the playground conveyed a message about teachers' need/right to watch children (Cannella \& Viruru, 1997; Greenman, 2007). Only the sleeproom where all the windows were curtained was an apparent exception, but it was fitted with a child monitor, and children were checked through the high window in the door if no teacher was in the room. In most other communal areas the provision of internal and external windows, along with the open doorways and latched back doors, made it easy for teachers to see what was happening through the centre. When I was in the quiet room looking at a portfolio with Jeff, a teacher who had been watching from the main room came in and asked Evie to stop taking pages out of her portfolio. The requirement that all furniture be fixed (to minimise earthquake hazard) restricted the use of storage units to partition areas within the main room, which contributed to the ease of supervision.

The internal windows also meant, unusually for New Zealand, that children might be in a room without a teacher, although they were rarely allowed to close the door, as they are in some Scandinavian centres (Einarsdottir, 2006; Test, 2006). When Evie went into the quiet room with other children and shut the door, a teacher quickly intervened, opened the door and talked with her. Teachers sometimes made use of the internal window to observe young but mobile children who had been placed in the Babysafe as a way of containing/protecting them: 
While we had lunch Macauley and David were in the quiet room, with the door shut, in the Babysafe. Had presumably had lunch earlier. Seemed quite happy, but Macauley was banging at the window once children were down from the tables. [22/9/05]

The lack of internal windows into the locker room and the children's bathroom made these areas more difficult to supervise, although the absence of doors and the low dividers between toilets helped teachers to visually check and aurally monitor what was happening. The only rooms in the centre that offered total privacy were adult-only spaces: the staffroom, the storeroom, and the adult toilet.

Supervision was also a key focus for teachers outside. The playground was Lshaped, and the number of teachers outside governed how they positioned themselves. Once there were two, they could separate out with one near the deck/sandpit and the other in the open area near the fort. While the fort was the most challenging area to supervise, some features such as the gaps between the fence palings, and the window and doorway into the space under the fort helped:

There is an altercation under the fort, and [the teacher] moves quickly across and climbs through the window to sort it out. [22/9/05]

Teachers' documentation reinforced their monitoring role. In the document headed General information, instructions included "SCAN SCAN SCAN", and teachers were punctilious in meeting this requirement. For example, no child was ever outside without an adult. There was no indication teachers questioned the assumed need for supervision. Indeed, some of the children's interactions reinforced the feeling that it was essential. At times, however, the supervision requirements appeared to constrain teachers. Did James T. understand why, when he asked for a swing, he was told he would have to wait until another teacher came outside? A fuller explanation was given to Cassidy when he asked for horses from the storeroom; the teacher needed to wait for another teacher to come back from her break before she left the room.

These ways in which the structural detail of the building allowed teachers to monitor children were anticipated in the literature on centre architecture (Walsh, 1996), and also in comment on philosophical traditions that permission surveillance of children (Cannella \& Viruru, 1997, 2004; Greenman, 2007). The implications of this high level of teachers' monitoring for children's curriculum experience will be revisited in the following chapter. But before that, the ways in 
which these same aspects of architecture and layout simultaneously promoted a sense of community will be addressed in section 5.4.

While this discussion has focused on children's experience, it is important to acknowledge the wider contexts. A further implication of the unimpeded sightlines, of which some children were aware, was that teachers could see when a colleague needed support. Comments by the management team in staff meetings also showed that observation was an inherent part of their role. And teachers collectively were open to scrutiny by a range of others: parents, Management Committee, visiting lecturers, health and safety officers, and officials from the Education Review Office and Ministry of Education. They were also constrained by regulatory requirements defining minimum ratios and supervision requirements.

\subsubsection{Teachers' control of resources}

Teachers' control of the potential range and use of resources was found to set parameters around children's curriculum experience. However, children often reciprocated with their own exercise of power, in both overt and covert ways, to redefine these parameters. Chapter 7 contains a more general discussion of children's resistance.

Unlike research in some settings overseas, where children must distinguish between resources for work and play (Apple \& King, 1977; Howard, 2002; King, 1978), here the majority of children's time was spent in exuberant free-flowing play. The few regularly required activities were in the context of the routines of eating, sleeping, toileting, mat-times and tidying. Although Alex probably felt it was work when a teacher insisted he complete a puzzle before he left the quiet room, for the most part children appeared free to direct their use of resources, and so apparently exerted considerable control. Evie and Walter took plastic pipes to the sandpit and filled them with sand, Sina applied her own facepaint, Evie, Aidy, Dora, Angus and Walter carried containers of water under the fort, Aidy made 'mud' by tipping water from the trough into the mulch, James discovered he could fit inside the new 'kitchen' cupboards. However, it was also clear that beneath children's freedom to choose and use resources there lay some perhaps less acknowledged messages about the level of teacher control. Observations showed teachers largely controlled what came into the play environment, which in turn 
influenced/restricted children's curriculum experience. Recognising this reinforced awareness of the ways in which power was embedded within the apparently benign discourse of choice and that freedom of choice was strongly bounded by teacher-defined parameters.

It was largely a teacher decision about what activities were set up. For the start of the day this involved providing access to familiar indoor areas, with books, blocks and Duplo, drawing, collage and playdough typically available in the main room. At other times of the day, and outside (as described in section 5.1.1), there was often more variation. Sometimes children asked for an activity, or their opinions were sought; during quiet time a teacher came to the door and asked what activities they wanted:

Maxine wants painting.

Mooloo wants drawing - she asks for colouring-in books as a second thought and [the teacher] says she is sorry she hasn't got any. Rex says "I want to come out" but [the teacher says she] is not ready yet. [10/8/05]

The closing off of spaces resulted in privileging/marginalising some play experiences. Resources that were kept in the main room were most frequently accessible. In contrast, the dress-up clothes, dolls and dolls' clothes were only accessible when the yellow room was open or if children asked for them. Similarly, the soft toys, cushions, the wider range of puzzles and books, and the Babysafe area and equipment were only accessible when the quiet room was open, although teachers typically brought a selection of books and puzzles through, and cushions were often set up on the deck. Outdoors, the siting of the sandpit in the covered area meant sandplay was privileged over experiences using equipment in the wider playground, such as swings, bikes, challenge course and fort, where access depended on the weather.

Teachers also defined how and where equipment could be used. Dolls and pushchairs could move freely indoors and out, but bike-riding and balls were defined as outside activities. While puzzles, books and collage were usually restricted to inside, teachers could, and often did, set up an activity using them on the deck. Younger children, and children new to the centre, needed to learn the ways in which it was defined resources could be used. (Rules will be discussed in Chapter 7.) Younger children were reminded that the Duplo stayed inside. When Fleur made a clay creation at the dough table, she showed it to a teacher: 
... and then heads towards the outside door with it. "Take it back to the table Fleur" says the teacher-and Fleur does. [15/8/05]

On other occasions the clay table was set up outside. At times the large plastic construction blocks were a source of contention. One day, after James and Aidy had carried several up onto the high platform of the fort, James was encouraged to go to morning tea, and while he was inside the teacher carried them down. When James returned:

He says very loudly "OOH, you shouldn't do that ". And then "We made that". Levi echoes "Yeah we made that". James jumps on a flat piece of waffle block and roars loudly. [8/8/05]

The following day James, Levi and Angus:

... are all carrying crates, working together and around each other. The lid comes off one that James is carrying up the steps. "Oh man" he says, and the others repeat "Oh man" several times. When they are all up there, they climb on to the boxes and shout. [9/8/05]

They also carried up a ladder, ignoring the ruling "No ladders"; a second teacher stepped in and the blocks and ladder were brought down. Here the dilemma for teachers was balancing the boys' enjoyment with concerns for their safety; with the situation complicated by the boys' determination to assert themselves.

Along with safety issues, inevitably there were other considerations that created potential tensions for teachers. Definitions such as 'the dough stays on the dough table' and 'up the stairs and down the slide' can always be justified, using rationales such as practicality, safety, finance and philosophy. Nevertheless such rules, typically decided and enforced by teachers, not only underline the teacherchild power imbalance, but also create areas of null curriculum. Keeping dough on the dough table means it is not explored as a contact adhesive in collage or carpentry, as a textural addition to a painting, or in combination with sand or bark chips. On the other hand, it is a rule that is professionally justifiable in terms of maintenance of the environment, economy, and conservation. Defining the stairs as the route upwards means children cannot experience the physical challenge of slide-climbing or compare the friction of bare feet and shoes on the surface. The underlying tension for teachers is that their support of children's open-ended exploration/discovery often conflicts with other aspects of their role, and that pressures and constraints inexorably lead to compromises. 
While it became clear that teachers exercised considerable control over the resources for children and how they could be used, again it is important to acknowledge teachers themselves were subject to constraints. Decisions made at centre level could also restrict what was available. An obvious example was the previously made decision not to have a computer for children's use which might be seen as restricting the potential scope of curriculum. There were also financial restrictions which limited what could be bought; the fundraising chart in the foyer showing funds needed for a new water trough was an explicit reminder of this.

\subsubsection{Children's resistance to teachers' control of equipment}

Children's actions and comments largely showed they understood teachers' definitions of how and when resources could be used but there were nevertheless continuing minor infringements. When Angus rode his bike through the line of cones used to define the deck-area as bike-free, another child called out to tell the teacher. Children only occasionally touched equipment defined as teachers'. On one of the first days observing, I noticed Aidy:

...standing on the second shelf of the block unit, looking at the tape/CD player - watching or listening intently. I stood alongside him and he gestured to the two levers that were down, and indicated that he could/would push them up. However he also made a comment that included the word "teacher" and I understood that he felt he was not allowed tobut that he wanted to. [27/7/05]

Later observations indicated it was a rule:

Sina says "They touched the stereo thing, that's why they're being naughty". [5/8/05]

Children made use of my inexperience; Rex asked me to retrieve something from his family wall-pocket:

I pull it out, a purple box labeled "Spyco" ... and I say "You had better ask a teacher-they know the rules". [6/10/05]

The teacher who was changing a child in the bathroom decided it would not come out.

Rules about not playing with resources during mat-time were both less adhered to, and less enforced. Children often played with pieces of Duplo, even when the shelves had been temporarily covered with a fabric throw-over.

I let Emjay draw in my book, and then felt guilty because other children were also doing things, and this was defined as a disruption-Robert A. and Cassidy were both playing with blocks on the shelves. [30/8/05] 
The music unit was sometimes turned to face the wall so the instruments were inaccessible. The water-cooler in the main room was apparently a source of continuing tension; for almost the full period of data generation it was covered by a towel which was hooked to the wall on either side. Teachers and older children were able to unhook it when they wanted to fill a cup.

Children responded to and resisted teacher-defined boundaries of curriculum relating to physical resources in three other ways: they asked for resources, reinterpreted the meaning and use of resources, and brought in their own. Children were able to ask for equipment that was not available. Aidy had music preferences:

I heard Aidy complaining when Hi 5 was put on for the other children, and after a track, he was there, still on the shelf, with a silver CD in his hand, saying "It's finished". The teacher said that they would have a few tracks before they changed it. [27/7/05]

The indoor storeroom was in adult territory and not visited by children so they were unaware of the full range of contents; one of the teachers considered making a folder of photographs of equipment to overcome this difficulty. Nevertheless, as children became familiar with the resources they asked for particular items; Cassidy knew the plastic horses were kept there. Outdoors, children were not supposed to help themselves to resources from the shed but were able to look in and see what was available when it was open, and sometimes went in with an adult:

Angus asked for the green bike ("the big bike") and got it, and Evie asked for the cart which the teacher thought about-and explained her thinking -in view of all the other vehicles out. In the end it did come out and was very popular. [11/8/05]

Although children could and did ask for equipment, both indoors and out, and teachers usually acceded unless it cut across the routines of mealtimes or tidying for the end of the day, it was nevertheless clear to children that the teachers held the power to make these decisions. As Cassidy commented to me one afternoon “They got no horses out". However, the teachers' responses to Aidy's request for particular music, and to Evie's desire to have the cart, are examples of the complexity of decision-making which constantly confronts teachers as they assess the individual's request within the context of their awareness of the wider group. For Aidy and Evie these were examples from a succession of tiny episodes through which they learned about the constraints of living within a community. 
Children redefined how resources were used. Often these momentary redefinitions were not commented on by teachers. Aidy crawled, dragging a length of green closed-cell foam in his mouth calling it "pizza". Other children, after watching him, looked for similar pieces [17/8/05]. James brought a coat hanger outside: ... which he holds with the wide end to his body, and the hook sticking out. He pulls the wide part—and then releases it (like a bow). [9/8/05]

Soon Rex was also carrying one.

At times children's redefinition of resources ran counter to teachers' definitions. For example, while there was a rule against making guns, children occasionally created objects with Duplo that seemed in the way they used them, to be serving the function of weapons. The sandpit shovels were regularly used by the older boys in free-flowing dramatic play across the playground.

Levi and James are using plastic shovels as guns- "bang bang" in and around the fort. [8/8/05]

Teachers observed but did not challenge this use. When an art activity with discs of paint, brushes and a jar of water was set up, Evie:

... was busy using the discs themselves on the paper, and involved in her own version of messy play. A teacher stopped her eventually and explained that the activity today was painting with brushes, but said ... that maybe we could have finger paint later in the afternoon. [30/8/05]

Often the way in which children redefined resources was affirmed by teachers:

Down the far end of the playground a new game has started using the plastic cart, which has been filled with medium and large rubber balls. Each ball is taken out, sat in the open space in the handle, and then James kicks it strongly and it flies up into the sky. James roars with each kick. Levi is sitting on ... the challenge course watching, he begins to join in with the roar of acclaim.... When the teacher returns her voice joins in too. [22/8/05]

Later, the teacher brought a wooden catapult out of the shed so more than one child could participate.

There was uneasy tension around who might bring new play materials into the centre. Notices on the whiteboard reminded parents:

PLEASE: as stated on previous occasions, children are not to bring toys from home to centre. It causes children to become upset unnecessarily if they have to be removed or get broken. [9/8/05] 
Children did bring items from home, and were often discreet about them, as Corsaro (2003) reports. I noticed Luke had a little orange truck that kept appearing in his hand and then returning to his pocket. Later I saw a teacher telling him to put it in his pocket. She commented perhaps she should have taken it and put it in his family's wall-pocket, but she felt that went against the goal of building links with home. She continued:

"If children can bring things, and have to work out that they need to share them, or to keep them safely hidden away, then this is useful learning." [17/8/05]

A more familiar reaction was when a teacher took possession of a dinosaur when she found it came from home. It was older boys who most frequently were seen showing secreted items. These included coins, a whistle, a plastic dragon, a fighting figure, a fire engine, a car and caps for a cap gun. There was a fascination with items children showed in secrecy to others, which often seemed rewarding and empowering to the owner, and so a way of gaining status with peers, as Corsaro (2003) reports.

A further tension was that some children's resources were accepted as legitimate. Fleur had a small piece of cloth she sometimes carried around with her even when she played, Jeff carried his own doll. Children also found ways to bring in possessions overtly. Rex wore his new watch. Maxine wore a silver tiara, and when I asked about the rule of not bringing things from home she said:

"Yes you are as long as you are careful and look after them"-so much for this week's notice to parents in the front hall. But maybe it counted as a headband rather than as a crown. [10/8/05]

Older boys also often wore clothing with emblems of Batman or Spiderman. There was also provision for children to bring items to show at mat-time on Wednesdays, and for a period there was a box for them in the foyer:

Rex and his mother arrive. He has a pair of plastic handcuffs. He runs out and shows me, and then a group of children, and then [the teacher]. His mother later tells me that he has brought them for news. And that they are "in the news box". [18/10/05]

The most exciting object that came from home was a replica of a crocodile's head. James's excitement in sharing at mat-time was infectious:

The teacher, [with James standing beside her], talks about it, how James's mother brought it home from Australia, and how "when I first saw it I thought it was a real crocodile", touching it. Unconsciously James lifts one leg up and down like a horse pawing the ground, while he holds it. [7/10/05] 
The teacher focused mat-time around it, sang a crocodile song and allowed every child the chance to touch it if they dared. Such a public event seems likely to have brought James even more recognition than privately sharing a secreted item.

The prohibition on children bringing items from home could be considered to be in tension with teachers' professed commitment to supporting children's interests. Implied within the ruling was a prioritising of adult identification of children's interests, over children's own judgement. Rex's interest in computers and his wish that he could use one in the centre provided one clear example of adults' decisions taking priority. However, while Rex may not have succeeded in expanding the boundaries of curriculum to include his particular passion, this discussion has begun to show the active role children took in renegotiating the teacher-assumed scope of curriculum.

It must be acknowledged children also sometimes exercised control over their peers' use of resources. Alongside the many examples of children being inclusive and accommodating, there were occasions when a child defined how equipment could be used or who it could be used by:

James runs up to Maxine who is on a bike, and kicks the wheel of her bike. $\ldots$

\section{Maxine climbs off and James climbs on. [22/8/05]}

Sina said Fleur was too little to wear the elephant costume, and Aidy was told Thomas the Tank engine was "a baby's toy". Children sometimes also limited others' access to spaces. Cassidy and Evie rejected Robert. T.'s attempts to join them in the little tent. Aidy, standing on the steps of the fort, said to Jeff:

"Get away from that or I'll get mad" and stands menacingly across the steps blocking Jeff's way, and with a fierce expression on his face. "Get away" [15/8/05]

He is persuaded to let Jeff up but announces: "No more big kids, I'll lock the door".

\subsection{The importance of relationships and community}

Observations showed that while windows were a mechanism of surveillance, they also served another quite different purpose; they enhanced the sense of community, of children and children, and children and adults, co-existing together. The only windows that standing/walking children were unable to look out of were the high windows in the locker room and bathroom, and in the laundry 
and sleeproom doors. From every other communal space, children were able to watch others, in other areas of the centre, from behind the security of the glass. Even in the sleeproom Aidy, waking up, sat on his mattress and raised the curtain to watch children outside in the playground before he chose to emerge. Being able to observe others, without necessarily engaging with them, seemed particularly important for younger/newer children. Younger boys watched older boys in the quiet room, observing without needing to be involved. Aidy (aged 3), still experimenting with how to use the camera, chose to photograph children outside through the window. When a teacher wanted James to help her pat children's backs in the sleeproom, she lifted the net curtain and beckoned to him. Younger children could retreat to the Babysafe yet still watch what others were doing from behind the fence. I noted it was:

A space that is theirs but not separated from the others. Still able to see. That theme of 'being able to see' as well as 'being able to be seen' is very strong everywhere. [21/7/09]

Older children could observe younger ones in the Babysafe. At a quiet time I noted:

Walter seems bored by the stories, but he finds the babies interesting entertainment. [1/9/05]

These same features which allowed children's activities to be constantly monitored also enhanced the sense of community; this second function was not one I met in the literature.

There were other features of the environment, particularly the displays of communal artworks, the posters recording centre events such as the walk to the construction site, and the family photographs on the fabric wall-pockets which also enhanced the feeling of community. Children were aware of these; when Robert A. found his photograph was no longer displayed in the quiet room:

He says "It's my last day but I need to be there". The teacher explains that they took the photo down so that it could go into his portfolio. [16/12/05]

The way teachers set up the environment enhanced the feeling of community. Equipment, such as the round tables, two-person art easel, large water trough, large tyre swing, and wagon encouraged children's collaboration. Multiple chairs were supplied at each table, multiple bikes and ride-ons allowed children to ride as a pack: 
I really got a sense of the joy of riding fast-they sped after each other, turning fast, sometimes following close, sometimes taking individual routes, but weaving an individual dance in and around the cones and over the ramp.... I could sense the exhilaration of speed, of skillful riding, of pleasure in their collective swooping and turning. [11/8/05]

Large supplies of sandpit and waterplay equipment promoted group play. Unlike Montessori settings where tasks convey the message of solitary work, here almost every feature of the resourcing encouraged children to engage with others. The open layout, with furniture restrained to the walls, supported awareness of and conversations with others in the same room. When James was at the table with foam blocks he called "Let's make a city" to another child, across the room, repeating it four times. When one teacher removed a piece of chalk from Macauley's mouth another who was watching recalled:

... an incident at a centre party in the dark where she was given a sweet, which she put in her mouth — thought how strange, and then realized it was a piece of chalk.

I noted the "sense of community" with:

... interactions ebbing and flowing backwards and forwards across the room. [27/9/05]

These aspects of the architecture, the layout, and the furnishing which allowed for surveillance, were also the aspects that promoted a sense of community across the group. Yet they were also features that made it more likely that a child be disrupted in a self-appointed task. When Evie noticed the plastic blocks were not properly connected she went across to fix them but Charlie was adamant she did not want help.

Observations suggested the importance of increasing children's awareness of each other. I found children who attended on the same day did not necessarily know each other's names:

Jeff and I are talking in the playground. He looks across at the truck that Evie is sitting on, and tells me "That girl's having it". I ask him "Do you know that girl's name?" and he says "No". I tell him it is Evie. [15/8/05]

Having observed Fleur through a day of delighted companionable play, I heard a teacher tell James's mother his had been "an o.k. day". This led me to reflect on how rarely he had appeared in my observations of Fleur. Early in the morning, he and Aidy had come briefly to the table where she was doing a puzzle, but she had apparently remained focused on her own task. Once the outside doors were opened she, like James, had moved outdoors. There she and Mulan had played as 
a largely self-absorbed pair moving together around the playground. Later, after having her nappy changed, when Fleur carried her doll across to the swings, I had noted:

There are a group of boys in an enclosure made from the plastic crates, and with a sheet draped over it. They are shouting loudly but invisible.

The boys are bursting out of the crate house with much shouting and I hear "We're going to get the girls". Several of them-including James-leap onto the crates until they are broken apart and lie scattered on the ground.

Fleur appears unconcerned by/unaware of the noise and puts the doll in the swing. "'Wing” she says as she pushes her. Mulan runs out and joins Fleur. [15/8/05]

At lunch Fleur had sat at a table and James had eaten school lunch on the mat. After lunch she had slept, James had not. Her only close contact with him had been at afternoon tea, when she had sat at a table between him and Jeff and he had handed around the food. However, her attention had largely been on Jeff's mother. Through the rest of the afternoon their paths had not crossed again, and James had left before she was picked up at $5.50 \mathrm{pm}$. Although I had been aware of James at times, these observations suggested how peripheral he had been to Fleur's activities, and underlined how very different children's experience of a single day might be.

Children's responses in the context of the research strategies reiterated the significance of people. Despite my opening prompt to photograph favourite/special places in children's photo tours (Research strategy 2), many children chose to photograph people. In total 24 photographs were close-ups of a person/people, 55 were pictures of people in a centre context, and only 35 photographs were of places or resources with no people or only distant/peripheral people. Individual children's photo tours often showed a similar focus on people. In Rex's first photo tour his first six photos included two of Dora, two of a teacher, and one of Cassidy. Charlie (who was inside because she did not have a sunhat) took photographs of my feet while she worked out how to use the camera, and then:

... photographs of Evie, of Dora, and Mulan.... She also took a photo of Cassidy who was heading out the door, [and] of me.[1/11/05]

Her final photographs were of books, a puzzle, the playground through the window, and then she walked outside and took a photograph looking under the fort. 
One possible explanation for the focus on people is children's familiarity with the concept of photographing people, although the fact my other resources largely used photographs without people might have worked to counteract this. A more likely explanation is that the open-ended process allowed children to photograph what they saw as significant, and their results showed this included people. Einarsdottir (2005) reports Icelandic children, instructed to photograph important things in the playschool, also included others, particularly children. Here both teachers and children were included. The significance of other people, and of your relationship with them, echoes others' recognition of the centrality of people. Paley (1986) identified friendship and Corsaro (1997) social participation as central concerns for young children. Within more recent New Zealand research Brennan's (2005) ‘children's longing to belong' and Alcock's (2007) 'peer togetherness' reflect a similar orientation. The significance of people and of being part of a community in this setting will be expanded on in the next chapter.

Because each photo tour was an open-ended process where my research agenda and the child's own agenda were both 'on the table', some tours took on their own character with the unfolding process providing more insights than a tally of places or people. For instance, Grace's photo tour was an expression of being Maxine's friend. She chose, and checked that she was choosing, the same sites to take her photographs. In a reciprocal way, when I returned Maxine's photographs to her and asked: "I wonder what is special about this place/this one" she responded using "we":

"We hide from [teacher's name] monster there." "We can make food with the mulch." [4/8/05]

This finer level of analysis has not been reported by others using photo tours.

In selecting photographs for the like/dislike activity (Research strategy 3) I had largely avoided showing people. Yet frequently children's comments drew my attention to people. Some, like Cassidy asked "where's me?" when they first looked at the photographs. Evie used it as an opportunity to talk about her family:

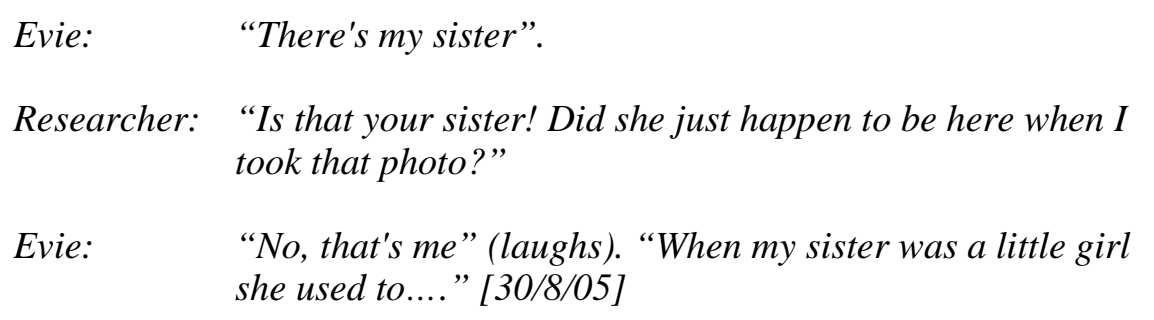


As I transcribed Evie's responses to the photographs, I noted "the significance of people-this is what she is commenting on". Robertson (2006) describes a similar realisation when she was showing children photographs of their experiences with musical instruments:

In a flash, rather like a small movie, I recalled all those other times I have sat with children with photos and patiently sat through the 'this is me; that's you; where are you?' comments, just letting them pass through this stage and waiting for the real thinking to emerge. As I re-viewed this 'movie', I realised that what I had thought was a preface, was in fact central to the entire movie. (p.154)

Children demonstrated the same focus in this research, even when my prompts and the photographs I supplied directed their attention away from people. This focus on the importance of relationships and of being part of a community will form part of the second emergent theme.

\subsection{Conclusion}

The opening section of this chapter contained a description of the current scope of curriculum in the centre. The primary focus of the following three sections has been to describe ways in which an adult-child demarcation was reflected in the architecture and resourcing of the centre, and so to introduce the first argument of the thesis, which is that this demarcation was a fundamental source of boundaries to children's curriculum experience. This discussion has identified some of the tensions that led teachers to define and control the parameters of the environment, and how children both acceded to, and resisted, teachers' controls. The demarcation between adults and children underpins many of the assumptions touched on through this discussion: the distinction between adult and communal places and between adults' and children's resources, the scarcity of 'real' tools, the assumptions around children's tasks, and the relative levels of power exercised. On occasions when teachers and children worked together-to plan for the 'barbecue', to make an underwater mural—or when teachers used children's resources for their own purposes, the adult-child demarcation was blurred. Those moments were like jolts in the data for the sense of adult-child community that each engendered. Broström (2006) repeats warnings against over-adherence to an approach which allows children to follow their own leads and inspirations and advises: "To avoid such an isolation from the adult's world, the teacher must consciously reflect on the relationship between a child's perspective and an adult perspective, and consequently create a curriculum that integrates them both" 
(p.239). Those moments when teachers and children collaborated suggested the possibilities of such an approach.

The notions of control and community have emerged through this chapter, and will be addressed in more detail in the following chapter which takes the lens of roles and considers the roles of both teachers and children in the centre. 


\title{
CHAPTER 6
}

\section{HOW TEACHERS' AND CHILDREN'S ROLES INFLUENCED THE BOUNDARIES OF CURRICULUM}

\author{
Power creeps in on little cats' feet. (Kincheloe, 2004, p.7)
}

The notion of roles, the sensitising concept borrowed from activity theory, was the perspective that framed the analysis for this chapter. The division of labour in a centre community may seem an unusual starting point for a discussion of curriculum, but taking this vantage point provided further insight into how children experienced and enacted the boundaries of curriculum.

The influence of the adult-child demarcation on curriculum boundaries, introduced in Chapter 5, is a continuing theme. In the first section of this chapter the teachers' roles, as children experienced them, are described and it is shown there was potential for these both to embed and blur that demarcation. In section two the second emergent theme of the thesis is introduced, and the focus shifts to the importance of becoming an accepted part of the community and to the contingent notion of exercising/experiencing control/power. It will be argued that establishing relationships with others was the central concern for children, but that this was frequently associated with expressions of control/power over others, and that these two linked concerns formed part of every child's curriculum experience. The third section describes roles children took as friends, as girls/boys, and as peer learners/teachers. Examples show the breadth of ways in which children enlarged the scope of curriculum, but also demonstrate how the dual concerns of relationships and control/power were evident in many peer interactions. Finally, it is argued that teacher-child demarcation in roles had unanticipated effects on older children's conception of themselves as learners.

In delineating the teacher roles, the data came primarily from the first of the two elements of children's perspectives described in section 1.1; the categories were derived from analysis of interactions between children and teachers over the five months, and were 'taking the perspectives of children' based on the researcher's interpretation of how it might be for a child in this setting. Children were not asked to comment on teachers. 
The additional research strategies referred to in this chapter, beyond those described in Chapter 5, are the picture questionnaire (Research strategy 4), children sharing their learning portfolios (Research strategy 5) and the New Kid book (Research strategy 6). (See Chapter 4, section 4.4.3 for descriptions of these.)

\subsection{Teachers' roles from the children's perspective}

Children were aware of rostered roles teachers took: who was outside, who was on nappy-changing. However, analysis of observational data of children's interactions with teachers suggested different categories (see Figure 6). There were five clusters of ways in which children experienced teachers:

- the warm and responsive companion

- the day-to-day carer who looks after me physically

- the initiator and controller of resources, activities and events

- the monitor of the social and emotional dimension of the community

- the documenter of children's learning.

These categories are useful as signposts indicating patterns of behaviours but in reality, teachers shifted seamlessly between these. This section outlines these roles, and suggests implications they had for children's roles, and for the scope of curriculum. It also provides examples that will be used in introducing the second emergent theme. Others have categorised pedagogical strategies teachers use (e.g., MacNaughton \& Williams, 2004) and textbooks for early childhood teacher education students offer overviews of teachers' tasks. What this analysis of teachers' roles contributes is a consideration of how children experienced them. While the analysis would have been strengthened if children had been asked for their thoughts, only Einarsdottir (2007) refers to undertaking a similar line of analysis, but without reporting findings. 


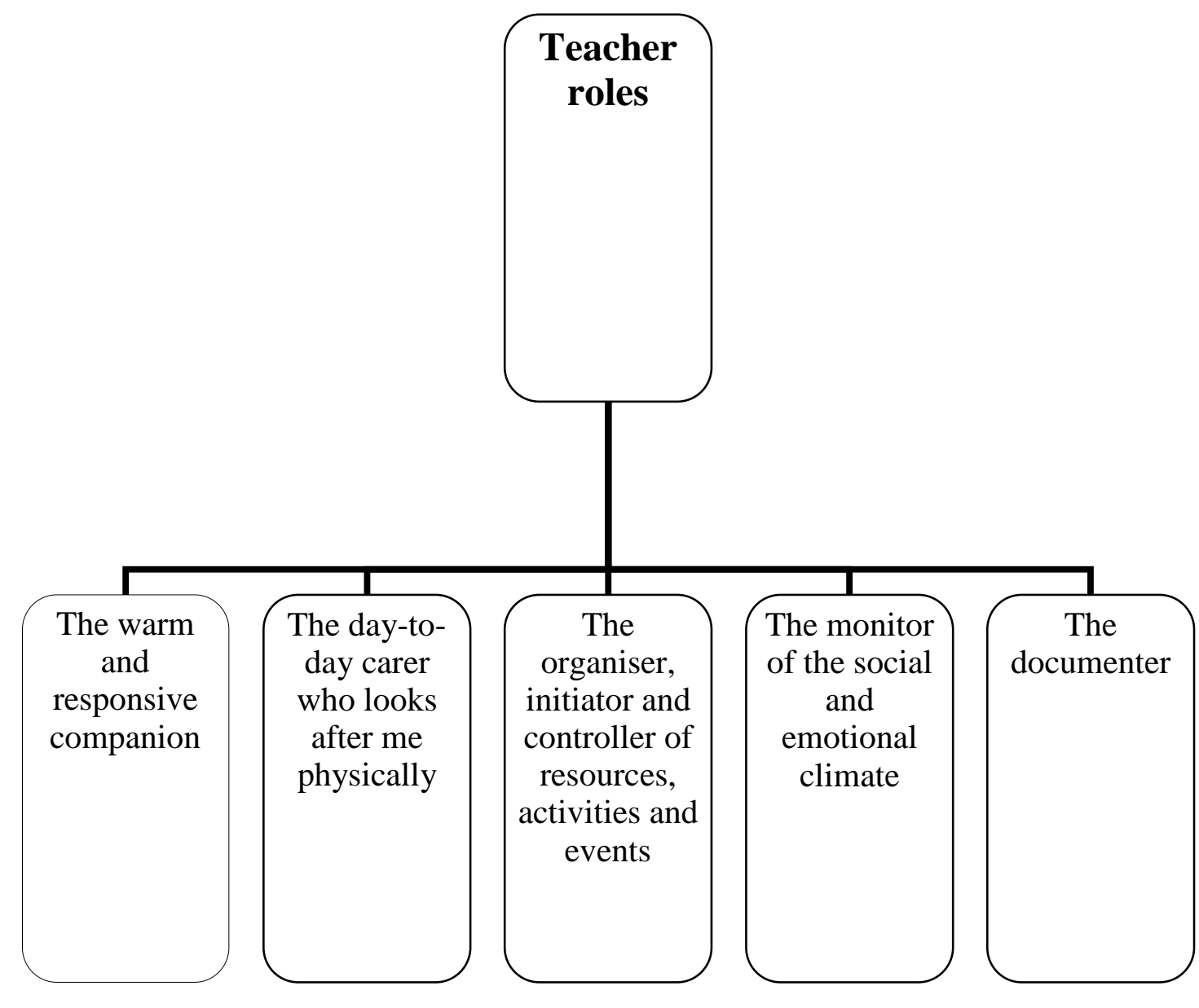

Figure 6: Teachers' roles, as children experienced them

\subsubsection{Warm and responsive companion}

Teachers' habitual modus operandi, in all roles, was to speak positively and enthusiastically, and this was most marked in their role as warm and responsive companions. Whenever children chose to approach teachers, they did so appearing sure they would be responded to warmly:

Fleur says: "I've got a sore knee".

Teacher: "Yeah, where's your sore? Can I see your sore? Did you want me to help you?" [as Fleur pulls up her trousers]. [26//9/05]

Children used teachers' first names, hugged them, sat on their knees, indicating they understood they were enjoyed and valued. They invited teachers to join them, and teachers in turn approached children at times and in ways they judged appropriate. Sometimes it was to engage a child:

James was wandering at a loose end and the teacher invited him to sit down in front of her for a massage. Others joined in, and she soon had everyone but Cassidy in a line stretching in front and behind her. [20/10/05] 
Often it was to deepen children's interest in their play. When children were blowing bubbles in the water, the teacher said:

"I like the way they all join together. If you look at them, can you see the colours that are moving on top?" [22/8/05]

Children responded when teachers asked questions or contributed new information and seemed to accept these as a seamless part of teachers' responsive companionship. When there was a need for teachers to coerce children, and this role shifted towards the role of monitor, teachers' typical first response was to intervene with humour and playfulness, as Brennan (2005) also noted.

Children usually delighted in teachers' forays into their activities. A few seconds of engagement could spark off new directions in the play. When Robert T. and others were riding bikes, the teacher:

...steps in front of the bikes in turn, with her legs spread wide, and then pulls back onto the grass at the last moment....Robert laughs a lot. [28/9/05]

Later he stands with his legs spread, stepping back from the bikes just as she had done. There were occasional moments in the busy environment when the shared interactions of a child and teacher radiated their mutual delight. These were moments of loving intimacy:

Macauley [just one year] and [a teacher] play with a tractor in the centre of the room. He holds it, she names it, she runs it through his hair, he holds his hand out for it, then runs it through his own hair. He sits snuggled in against her looking very comfortable. Later they play a game of alternately crawling towards and away from each other. He looks filled with delight. [19/8/05]

Several children photographed teachers as part of their record of favourite 'places' in the centre, which reiterated their significance. Brennan (2005) also found that "teachers devoted a significant amount of time and energy to establishing and taking care of their relationships with children" (p.110).

This role offered the greatest potential for blurring the adult-child demarcation. It allowed teachers to work alongside children, as when teacher and children created a sand mountain together. It also implied a reciprocal role for children as teachers' companions; Fleur found a teacher's shoes and carried them inside for her.

However, teachers' unwavering acceptance was potentially also a source of power for some children, and their positive attitudes invoked Scutt's (1992) question of 
how beneficial it is for children to grow up with perpetually unselfish, cheerful female role models?

\subsubsection{Day-to-day carer who looks after my physical needs and wants}

Analysis of data led to the (unfashionable) separation of the care and education aspects of the teachers' role, because it seemed children rarely associated routines with learning. The youth and dependency of some children, and the assumptions around older children's competencies and roles, meant caring took up a large proportion of teachers' time. Children's role within routines is described in more detail in Chapter 7.

This teacher role carried potentially ambiguous messages for children about their own role in the community (Singer, 1996). The fact that the teachers and the cook did most of the tasks associated with routines unassisted carried a double-edged message. As was foreshadowed in Chapter 5, it could be read as disempowering for children because it implied 'real' tasks were adults' work, and so discounted their role as contributing members. However, it could also imply that teachers were here to do the work for children, thus privileging children. Occasionally an undercurrent of power in children's reactions suggested this latter interpretation:

The food comes around, and James takes charge of handing it around. James shouts out "Where's the scrap bowl" and a teacher comes over with one. [22/8/05]

\subsubsection{Initiator/organiser/controller of resources, activities and events}

The multi-faceted role of teachers controlling the environment was described in Chapter 5. Here, that role is extended to include their role as initiators/ organisers/controllers of activities and events. These included both familiar events like mat-time, and more atypical ones such as walks outside the centre. This role is described in detail in Chapter 7.

\subsubsection{Monitor of the social and emotional climate}

The role of overseeing the social and emotional life of the community had high priority for teachers. It was reflected in teachers' frequent references to children's social skills in planning documentation, but a larger part of the role focused on managing children's behaviour, particularly children's conflicts. Observations 
indicated this was a role in which teachers felt less confident. The word 'monitor' was chosen to convey the sense of overseeing but not necessarily controlling.

Teachers were understood to be the source of comfort; children turned to teachers to protect them from physical and emotional threats and when they were sad or hurt. However, there was also recognition that teachers could not provide total protection. Sometimes a teacher was not present or was unable to stop the aggression immediately. It seemed children might have come to accept that a level of aggression among children was the norm.

Teachers consistently provided models of peaceful interaction, coached children in how to respond to confrontation, and supported them in potentially challenging interactions. The many observations of children using such strategies showed the effectiveness of this modelling. However, teachers' efforts were particularly challenged by children's aggression. It is tempting to gloss over this but a continuing level of conflict among children, particularly among older boys, was a reality at this time-it was reflected in teachers' discussions, in what children and parents told me, and in observations. At times teachers seemed powerless to prevent such episodes, and were left to comfort the victim, and remonstrate with the aggressor after the event. Handling children's conflicts was a central issue at this time for teachers.

\subsubsection{Documenter of children's learning}

Teacher as documenter of children's activities was the least obvious of the roles, and was one where the demarcation between adults' and children's roles was most marked. Children knew teachers watched them, wrote notes and photographed them, but this appeared as only a minor activity in their busy week. Yet, although teacher as documenter was the least visible of the five roles described, in turn it was the most visible aspect of a broader role of teacher as educator (see Figure 7). From my work as a lecturer, I knew this aspect of being a teacher was pre-eminent within teacher education, yet here much of this role appeared to be hidden from children. Discussions of children's interests and learning happened in the staffroom, often as part of the monthly planning meeting, which was also where centre programme decisions were made. Individual teachers set goals for children's learning, and these were shared amongst the team. However, none of this information was routinely shared with children. Even updating portfolios and 
making posters of centre events were never observed being done with children, although children were sometimes asked if they wanted an item added to their portfolio. Just as children did not know about washing happening in the laundry because it was at a time and in a place where they were not present, so much of the role associated with documenting their learning was invisible to them.

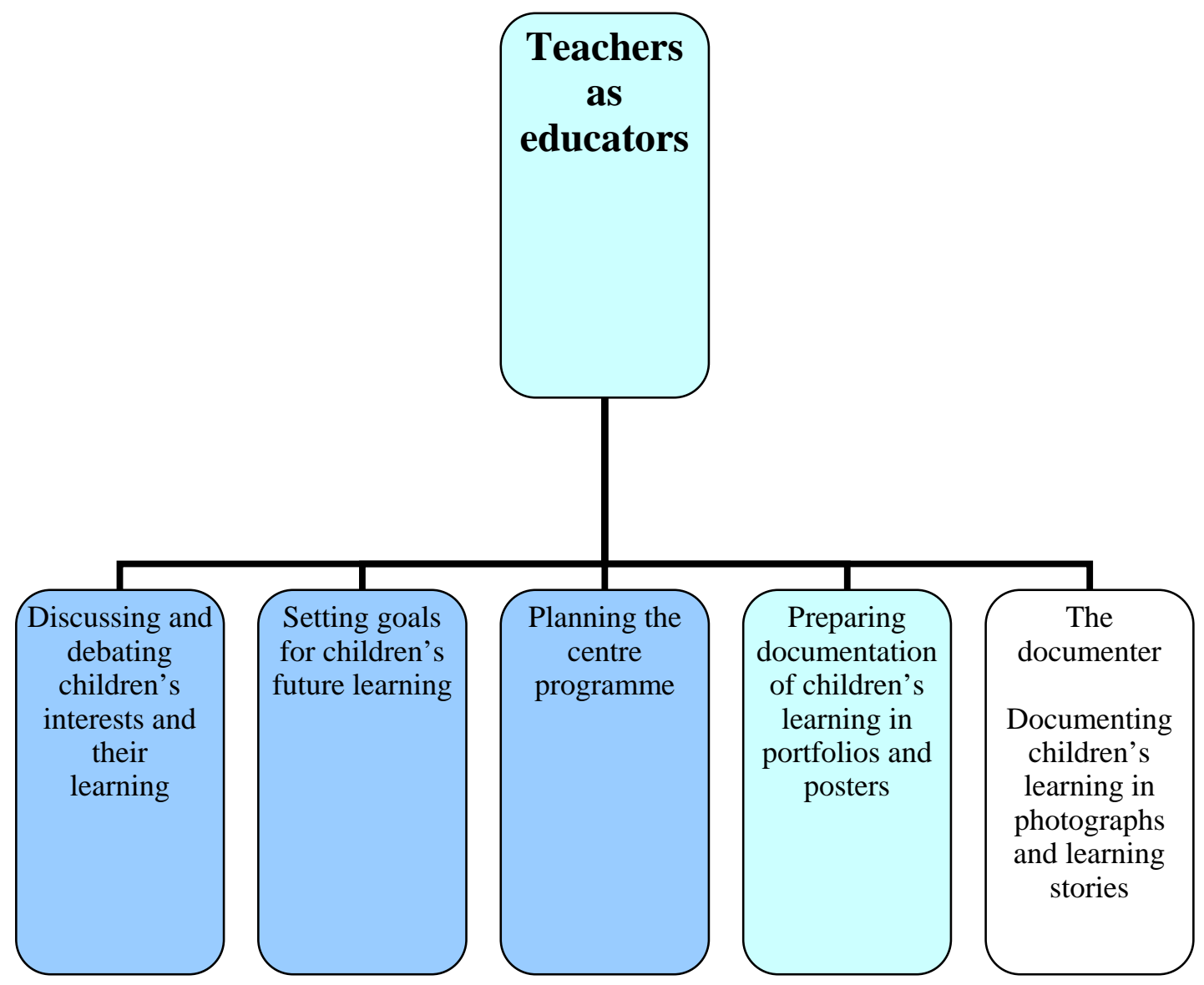

Figure 7: The visibility of the teacher's role as educator (Shading indicates the visibility of the role to children; heavily shaded aspects of the role were less visible.)

The most obvious consequence of this disjunction between children's perceptions and teacher purpose was that children were unlikely to know what had been identified as their interest/s and what it was intended they would learn. This diminished opportunities for children to be partners in their own learning. However, there also seemed to be implications for older children's perceptions of themselves as learners. This topic is revisited in the final section of this chapter.

How did these teacher roles influence the boundaries of children's curriculum? Most obviously, they implied contingent roles for children, and only for the warm and responsive companion role was there broad overlap between the teacher and 
child roles (see Figure 8). The other four roles all implied teachers held control and the reciprocal child role was a less powerful one as the one-cared-for, the onewho-participates, the one-monitored, and the one-who-is-documented. Identifying teachers' roles and implied children's roles underlined how the adult-child demarcation permeated assumptions about the division of labour. In reality, the adult-child demarcation was blurred at times in all these roles, although the balance of power still clearly rested with the teachers.

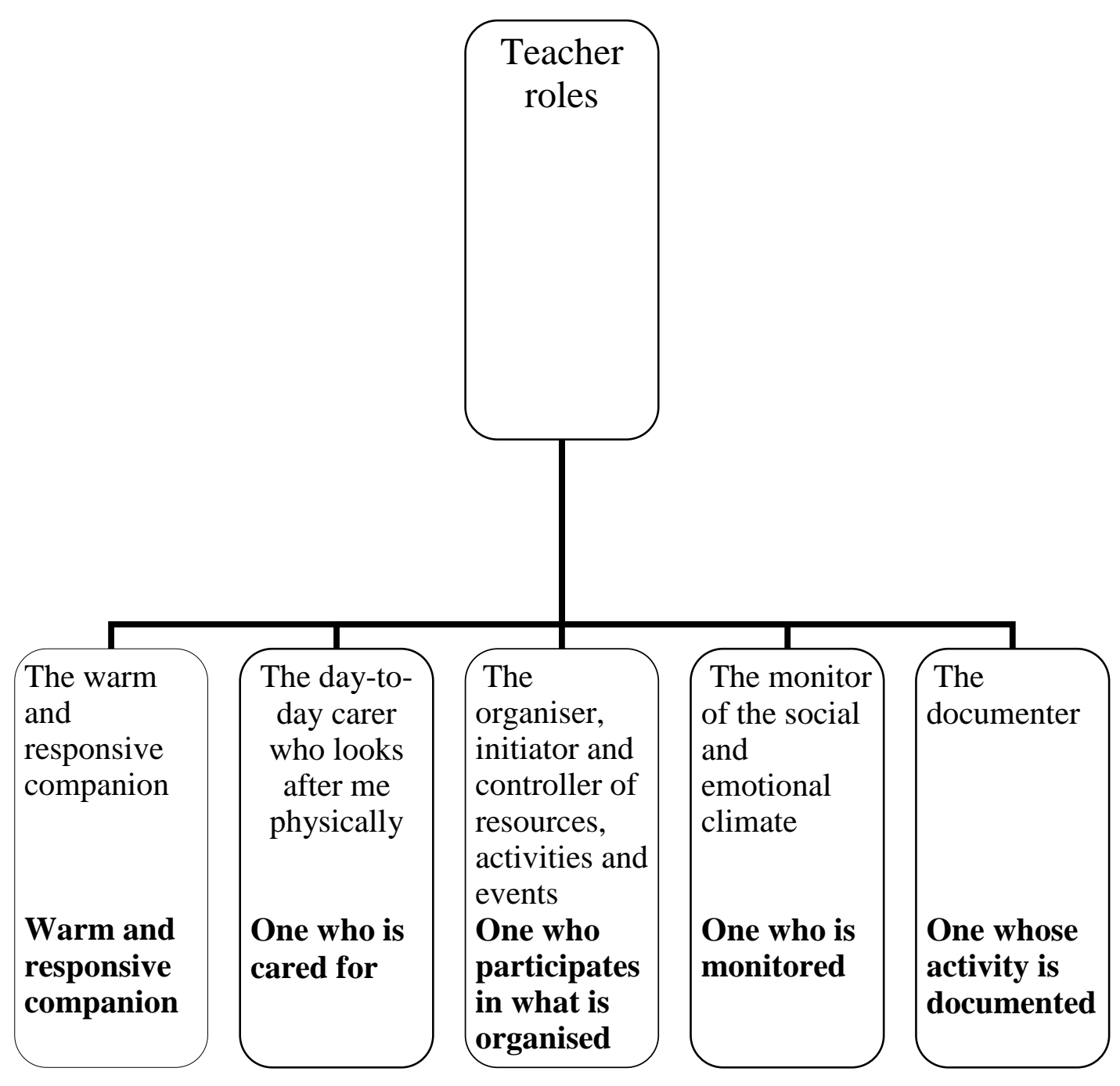

Figure 8: The roles implied for children by the teachers' roles

The predominant focus for teachers through these roles, although less so in their role as documenter, was on maintaining a smooth-functioning and harmonious community, as Rivalland (2007) found in an Australian study. This focus led teachers to adopt two modes of behaviour that sat in an uneasy juxtaposition. The notion of the dialectic (Goulart \& Roth, 2006) is used here to capture the struggle to mediate these contradictory but co-existing elements within the teacher's role. 
On the one hand teachers promoted and demonstrated empathetic companionship and participation within the harmonious community, and on the other hand they exercised control/power in order to ensure the ongoing functioning of that community. The high proportion of time teachers spent in the role of warm responsive companion suggested this was their preference, but children's words and actions repeatedly prompted them to step out of this role. Typically this was done in a 'light' way through distraction or with a playful comment, and when teachers did use more overtly powerful strategies, their actions and words often suggested their discomfort. A teacher shared her embarrassment after she spoke loudly and angrily to a child disrupting mat-time [19/8/05]. Her reaction indicated the uneasiness with which the two dimensions of maintaining a harmonious community, and the exercise of power/control in order to achieve this, co-existed, and suggested the complexities mediation of this dialectic might involve. These notions were to be central to the second emergent theme of the thesis, which is introduced next.

\subsection{The second emergent theme-participation in the community and the exercise of control/power as curriculum}

Two threads consistently emerging through data analysis were the notions of participation within the centre community, and the exercising of control/power. In this section it is argued that because both were concerns of children, and constructs they were using to establish/define their identities within the centre, they were aspects of curriculum. There were three steps to recognising this. The first was recognising the significance of relationships for the children, the second was identifying that the dialectic quality that characterised the teachers' roles was also a characteristic of many of children's peer interactions, and the third step was understanding that the two seemingly juxtaposed impulses of establishing positive relationships and exercising control/power were central curriculum concerns for children. These three steps are detailed below.

\subsubsection{Relationships and living within a community as the core of curriculum}

Analyses of four data sources converged to suggest that establishing relationships with others and becoming an accepted member of the centre community was the core of curriculum for children. These sources were conversations with children, with parents, observational data, and teachers' planning documentation. 
As described in Chapter 5, children's responses in the context of the photo tours and the like/dislike activity (Research strategies 2 and 3) indicated the significance of people, and conversations in the context of other research strategies reinforced this. When Rex shared his portfolio and selected pages to photograph (Research strategy 5), I noted:

What he stopped to look at was the photographs of people. [10/10/08]

Like most others, Amanda paused at the photographs; she identified children before adults, but did name those as well. Children also commented on and named people no longer in the centre. What interested Aidy particularly was his portfolio from an earlier centre:

He could name some of the children in the picture, and talked of them as friends. [12/12/05]

Children were also interested in photographs of themselves when younger. For Walter:

The first photo he took was of him very young climbing on a challenge course. He described it as "when I was a baby". [10/10/05]

Children did comment on details other than photographs of people, but only for Mooloo did the artworks seem to be the most significant aspect. These experiences suggested the importance of others, both children and adults. Some slight further confirmation came from the picture questionnaires (Research strategy 4). Although only four children completed this, all identified children they liked to play with, and two named children who were disliked as play companions.

Interviews with the six mothers offered confirmation of the importance of relationships; all readily identified children their child talked about. Evie's mother volunteered "She talks about James a lot". Fleur's mother listed children Fleur talked about, then added:

"She's pretty keen on [Teacher A]. She's always asking after Teacher A ... and there was Teacher $B$ who used to work here, and Teacher $B$ was one of the ones that used to look after Fleur and she still talks about Teacher B". [26/10/05]

Observations reaffirmed the significance of relationships. Many children spent a considerable proportion of time playing with a particular child or children, but also interacted with a wider range through the day. While a child might choose to play alone, this was typically a brief interlude in a companionable day. When 
children did play alone their actions frequently showed how aware they were of others around them. Descriptions later in the chapter of children's role as friend will provide further substantiation.

Finally, analysis of teachers' planning documentation also suggested the centrality of relationships. As explained in 5.1.3, teachers drew on children's identified interests in defining the collective focus for each 6-week period, but planning documentation for individual children often showed a quite different emphasis. I had assumed planning would relate to children's interests, and was initially surprised that many goals for individuals were focused on social behaviours, such as turn-taking and developing relationships:

OBJECTIVE: To explore social concepts and relationships further with peers

STRATEGIES: A variety of experiences that encourage her to be actively involved e.g., playdough, stories, construction, puzzles. Discussion about certain rules for certain situations [Progpl12]

References to individual interests were much rarer:

Provide more props for dramatic play (new and interesting) supporting her interest in school, school uniform. [Nobk921d]

Analysis of individual children's planning documentation showed a predominant focus on children learning to live as part of the community, and on their relationships with others. Apple and King (1977) and Jackson (1968) describe children as being socialised into the student role; here it seemed the planning focus was on children learning to be members of the community. Ritchie (2001) argues teachers' goals "should include a focus ... on fostering children's social skills and collective endeavour" (p.137). This planning focus reflected a shift in that direction.

Collectively, these lines of analysis indicated learning to meet the challenges, strictures and rewards of living as part of a group was the core of children's experience and learning in this site. For me this had been such a taken-for-granted aspect it had become transparent. Taking a child's perspective helped me to recognise the significance of one's relationships with peers and teachers and also to understand the challenge involved in moving from the nuclear family into this crowded centre community. Early in the data generation I reflected:

There is huge complexity in social interaction going on here, in working out who you are, and how you operate in relation to others in this place. And yet we call this "care", as though they were passive beings within the situation. [9/8/05] 
It may be that when a centre community is harmonious and relationships are largely positive this level of social learning is buried. The written planning could then indeed focus on individual interests. But here, as this chapter will begin to show, I had the privilege of observing a setting where the underlying dissension and struggle among community members was visible. This brought to the surface tensions that are perhaps always present but not often so obvious. Here, children's learning to live as part of a community, to ride the tension between individuality and interdependence was overtly displayed. On the page, this looks simple. These were after all children under five and professionally trained teachers. In reality my presence at staff meetings meant I knew it was a complex, demanding situation which drew on teachers' professional and personal skills. For many children it was also complex and demanding, requiring them to absorb and then adjust their behaviours to reflect the often ambiguous and contradictory messages they received from peers and teachers.

\subsubsection{The dialectic quality of relationships}

The second step to understanding the theme relates to the quality of the relationships within the community. As the description of teacher roles showed there was a dialectic quality engendered by their struggle to mediate between the apparently irreconcilable impetuses of establishing sensitive and empathetic relationships and maintaining a harmonious community on the one hand, and the exercise of control and power in order to achieve this on the other. This dialectic quality was found to also characterise many of the interactions and relationships among children. Episodes demonstrating this are described in the third section of this chapter.

\subsubsection{Relationships with others and control/power as curriculum concerns}

The final step in recognising the significance of the theme came with understanding that not only did these two apparently contradictory dimensionsestablishing relationships with others in the community, and the exercise of control/power over others - characterise many relationships, but that these were constructs children were using in establishing their identities in the centre and so were central curriculum concerns for children. Forming relationships was the predominant focus for children and teachers. While teachers' concern appeared to be the functioning community, children's focus was on the moment-by-moment reality of relationships with others. This was the heart of their curriculum 
experience. The significance of relationships with others is not a new thought (Corsaro, 2003; McCadden, 1998; Paley, 1986). Within New Zealand, both Brennan (2005) and Alcock had noted it, and Lee (2005) calls early social sciences learning, which includes learning about one's role in groups, "the most crucial area of the early childhood curriculum" (p. 54).

While teachers' exercise of control was a subsidiary aspect of their relationships with children, and of children's relationships with each other and with teachers, nevertheless children's exercising of control/power made it a significant aspect of curriculum. This was not universal. Peer interactions were mostly positive and companionable and a few children were never observed attempting to exercise control over others in ways that transgressed centre norms, but every child was a recipient of others' exercise of control and everyone witnessed peers exercising control/power over both peers and teachers.

The label 'control/power' was chosen to indicate the range of behaviours observed. Children and teachers exerted control, using both verbal and non-verbal strategies, to maintain/gain control of resources, and to control the actions of others. The word power was added because sometimes children's strategies appeared to be an expression of exclusion not aimed at achieving any tangible goal, and occasionally they seemed an overt expression of dominance. In one circumstance, described in Chapter 7, teachers' controlling strategies also bordered on physical domination.

Identifying the exercise of control/power as a concern for children is not new. Corsaro (2003) names young children's second overriding concern, along with social participation, as "challenging and gaining control over adult authority" (p.118), a theme reiterated by Kyratzis (2004). Corsaro (2003) also notes two central themes that consistently appear in descriptions of young children's peer cultures: "(1) Children make persistent attempts to gain control of their lives and (2) they always attempt to share that control with each other" (p.118, italics in original). The analysis in this thesis follows Corsaro, therefore, in linking the notions of participation and control. Where this analysis differs is that Corsaro focused primarily on children's resistance to adults, and saw children's rejection of others as largely protection of their own play. McCadden (1998) makes a similar point by identifying children's need to be able to access people and 
resources as a central aspect of their relational morality. While Corsaro (1997)

does also discuss inclusion/exclusion, these are not central aspects of his argument. This analysis also differs from Corsaro in arguing that while the exercising of control/power was not apparently a concern of every child, it affected the scope of curriculum for all.

Critical pedagogy had alerted me to the potential power imbalance in the teacherchild relationship; but not to finding that the wielding of power would be a feature of peer interactions. It was only McLaren's (2007) description of the Foucauldian notion of power coming "from everywhere, from above and from below" and being "inextricably implicated in the micro-relations of domination and resistance" (p.209) that reflected the way power appeared to be woven into so many of the relationships. The descriptions here therefore present a less benevolent picture of children's interactions than has been described in other ethnographic accounts both in New Zealand (Alcock, 2005; Brennan, 2005) and overseas (Corsaro, 1985; McCadden, 1998). However, Australian scholars have drawn attention to such undercurrents of power in children's interactions (e.g., Dockett \& Fleer, 1999; MacNaughton, 2003b).

Having introduced the second theme of the thesis and explained the steps which led to its recognition, the final section describes how children's exploration of relationships, and the exercising of power/control in the roles they took, influenced the boundaries of curriculum.

\subsection{Children's roles}

This section examines ways in which the roles children accepted or defined for themselves influenced their individual and collective curriculum experience. Some of these reflected age/gender: older boys, school-lunch children, nonsleepers, crawlers. There were established friendships and there were looser and briefer alliances. Because relationships were central, people themselves were the most significant resources and collectively they constituted an inherent curriculum boundary in this site. As people came and went that boundary shifted; for example, the departure of the three oldest girls significantly altered the community. 
Individuals also selected roles: Evie was a self-appointed rebel, Cassidy was a horse expert. Sometimes children were allotted roles by others: Alex was often defined an 'outsider' by James, Robert T. was excluded by a wider group. The data constantly demonstrated the individuality of each child's curriculum experience; every child's experience was shaped by the role/s they had chosen, or been positioned into within the peer group. It will be shown that for some children this had a significant impact.

The decision to focus on children's roles as friends, as boys/girls and as peer teachers/learners was made because these were roles every child took, and because these roles demonstrated different ways in which children influenced the scope of curriculum. The first two roles frequently reflect the dual intertwining threads of the second theme. With the description of the third role the focus returns to the first theme, the adult-child demarcation.

\subsubsection{The role of friend}

Establishing relationships within the community was a predominant concern. For those who were older and/or long-standing attenders, the focus was on their peers. For younger, newer or less-frequent attenders, relationships with teachers seemed equally important. However, the underlying impetus seemed to be the same.

My initial impression of peer relationships was of a kaleidoscope of smoothly changing patterns, as groups ebbed and flowed through the day. Children mixed widely across age and gender boundaries, a process encouraged by teachers, and assisted by the fact children came and went at different times. There was a myriad of examples every day: Cassidy joined Maxine and Grace in one of their constructed 'spaces', Alex followed Maxine in a jumping game. There were also more enduring relationships which significantly defined some children's roles in the centre.

Conversations with parents and children confirmed observational data suggesting how challenging the social intricacies of relationships with others might be (Corsaro, 2003). Children were learning about living as part of a community, about having and being friends, and were often also learning about how power might be wielded within relationships. Parent interviews reinforced the impression that peer relationships could be challenging. Only Evie's mother said her child never talked about peers being difficult. Three of the other mothers named one 
child, and the other two named two and three children respectively. Their responses confirmed physical aggression was a feature of some interactions.

Three examples show three very different ways in which children's relationships demonstrated that dialectical quality as individuals mediated the complexities of establishing and maintaining positive relationships yet also ensuring their influence and ascendancy.

\section{A model friendship}

Maxine and Grace (both 4 years) provided the strongest model of friendship.

Grace attended two days a week, Maxine came three days, and both had been at the centre since they were babies. When they were both present, they were almost always together, and their families arranged for both to leave on the same day although one was starting school several weeks later.

Within their relationship, they presented a microcosm of the tensions being played out across the wider social group. Observations suggested they were exploring the tensions between meeting their individual desires, resisting the other, and yet not destroying the fabric of their friendship. This appeared to be personal learning, not often shared with others; what was apparent to others was a robust friendship. A conversation with Maxine indicated the friendship was not always easy. After she had completed the like/dislike research activity, on a day Grace was not there, I asked if there was anything she did not like about the centre:

"I don't like anyone laughing at me."

When I asked: "Do some people laugh at you?" she responded:

"Grace, Grace's a friend but she laughs at me."

Researcher: "She sometimes laughs at you?"

Maxine: "No she always laughs at me."

Researcher: "Does she?”

Maxine: "Yes all day long, all day long, all day long." [17/8/05]

A later observation while they were dressing up showed the kind of power tussle they engaged in, and their care to protect their friendship. Grace wanted a veil Maxine was using and when she was refused, she threatened:

"I'm not your friend and I won't play with you." 
She offered two substitutes, one after the other, but Maxine refused both.

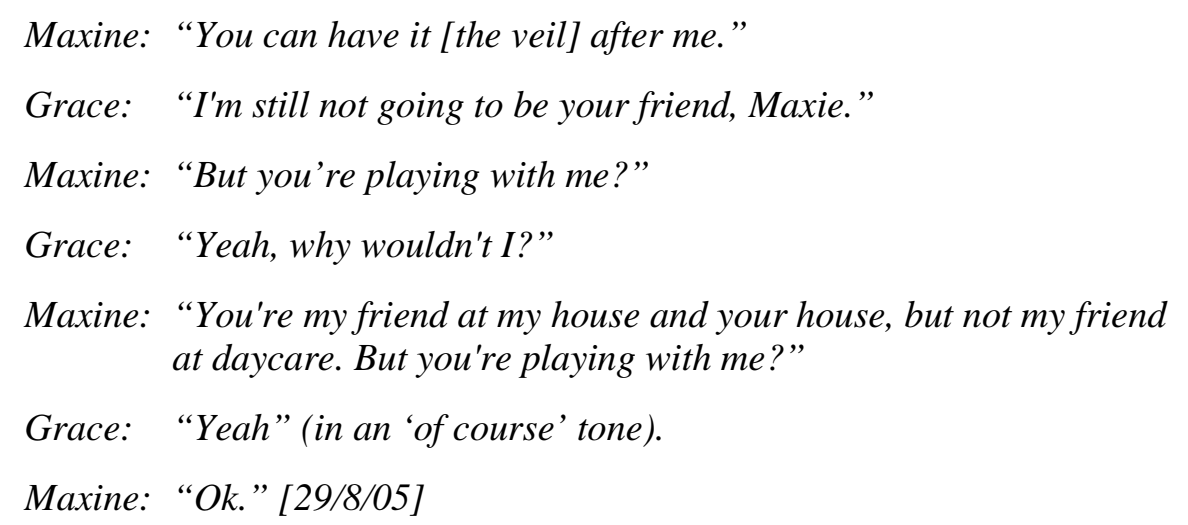

Often others were incorporated into their play, they willingly sat with others at meals, and superficial observations suggested they interacted harmoniously with everyone. However, conversations and observations showed there were preferred and avoided children. When completing picture questionnaires (Research strategy 4), they conferred quietly, and then Grace whispered that Sina, Evie and James were children she did not like to play with. Their lowered voices suggested they might see this as an inappropriate admission to be making. At times they rejected others in overt ways, but more often they expressed rejection in ways that were nuanced and apparently unnoticed. One day they were dressing up in animal costumes along with Sina, Amanda and Charlie. After Sina and Charlie left, Grace said:

"We go in the zoo because we're friends, eh?"

Maxine: "We're all going to have wings on."

Grace: "So we can all fly together."

Then Maxine says more quietly to Grace: "But Amanda doesn't have wings."

Grace: "So she can be in Sina's and Charlie's team." (Amanda is still in the room.)

Maxine and Grace put on the little net capes that serve as 'wings' and one of them says: "Bye, we're going to the zoo now."

They run out followed by Amanda. [8/8/05]

Some of my interactions with them, described in Chapter 4, had allowed me to feel at first-hand the experience of being 'othered' by them and this heightened my empathy for those children they had identified as disliked. On their last day in the centre, when they were adorned in glamorous dress-ups and with Barbie facepaints, I joined them on the mat to give them their farewell letters. Sina, who was Māori, came to sit on my knee as I talked. Maxine peeled a sticker off her letter, tried to stick it back, dropped it, and Sina picked it up and crumpled it, engendering Maxine's verbal wrath. I reflected: 
Knowing how Grace and Maxine feel about Sina made me sensitive for her-these two who already are parading the glamour of the Barbie-type white female, and she was in her ordinary old clothes, being screamed at by Maxine, and probably well aware they don't like her. [1/9/05]

This was one of two occasions when I wondered if there was a racial dimension within the interaction.

This friendship showed the complexity of balancing individual desires within the relationship. It also revealed the nuanced way in which they exercised power in their relationships with others. Each incident seemed slight, but I suspected the accumulated effect of the nuanced rejections could feel significant for Sina.

\title{
Playing with James
}

James (4 years) was a dominant figure, often the centre of running-chasingshooting games, and a significant focus for Aidy who spent a large part of his time in the centre aware of, and responding to, events James instigated. These three incidents convey the dislocated emotional quality of Aidy's interactions with James; there appeared to be no certainty in the kind of response an initiation from him would receive.

\begin{abstract}
Aidy has a blue cup in his hand and James has a green one. The water [in the trough] is blue and bubbly, so I assume it has detergent or soap in it. Aidy is drinking the water out of his cup. James is pretending to sip the water from his, but Aidy really drinks. A teacher, from nearby says "Don't drink it, you will get sick". James and Aidy both take a real sip from their mugs - James first and then Aidy. James continues pretending to drink, but Aidy takes several more real mouthfuls. His attention is on James, not the teacher. I sense that in drinking it, his focus is on James's approval.
\end{abstract} [19/8/05]
James has several waffle boxes on the high platform and is keen to take up the rest .... James carries the boxes up one by one. Aidy approaches with the big ball and says "I got a ball". James shouts "No". Aidy says "Hey James" and James says loudly "NO, No we don't need it". [8/8/05]
Aidy is on the digger. James pushes Aidy off the digger and he sprawls in the mulch crying. James kicks Aidy as he lies there. A teacher intervenes and asks what happened. [9/8/05]

Such observations suggested how gratifying having James as a play companion must be for Aidy. Rejections and physical violence only deterred him briefly. In contrast to the nuanced way in which Maxine and Grace exercised control/power, here the strategies were frequently overt and flagrant, simultaneously challenging both children and teacher. 
While the episodes with James showed how children could knowingly choose to ignore teacher-defined norms, Robert T. seemed to be still discovering the differing definitions of acceptable behaviour.

\section{On the cusp of being an outsider}

Robert T. (2 years) came two days a week. He was big for his age, was less articulate than many of his 2-year-old peers, and his clothing set him apart; he regularly wore elasticised boots and a red woollen hat pulled well down. He was a child who seemed at that time poised on the cusp of being defined as an outsider. The following episode suggests how difficult it was for him to decipher the different definitions of acceptability.

Robert had been involved in a sustained game on bikes with a girl and he had expressed his pleasure in smiles and laughter. Later he moved to the challenge course where Fleur was climbing:

Robert goes up the ladder, and pushes his face right into hers, leaning into her, while she is on the red triangle. Fleur leans forward and bites his arm. It does not appear to hurt. [28/9/05]

No-one seemed to notice, but a teacher called out to remind him which way to go and he retreated. He hit Fleur's bottom a few moments later when she was climbing, but there was no reaction or intervention. The girl with whom Robert had been riding joined them on the challenge course.

When she is half-way over Robert bites her. [The girl] looks across at me, but I don't intervene. She tries to climb down ... but Robert is holding her hand tightly and it is a few seconds before she can get away. When she is free, she stands, glaring [at him].

Robert climbed down and appeared to be going to join her, but she ran inside and he climbed on a bike.

Rex and Alex are playing with balls and Rex kicks a ball that hits Robert as he rides his bike .... Rex and Alex laugh and Robert looks emotionless, but reverses his bike away from them. He rides off around the circuit, and a moment later [a teacher] is saying in a loud and angry voice "NO, you are not to do that to people, that is not ok. You are not to do that to people. I am going to put this bike away." [They are the only people in the vicinity, and I assume he either hit or bit her.] She lifts him off it, and roughly puts the bike over on the grass against the fence. She tells him that he can't have it for five minutes. She seems really angry, at least initially.

Later he was allowed back on his bike. 
Robert $\mathrm{T}$ was a touchstone in revealing how difficult it might be for children learning how to develop positive relationships. He received conflicting messages, and was still deciphering what was appropriate behaviour with teachers and children, how to initiate positive interactions with others, and how to interpret messages he received. He attempted to initiate interactions, using models he saw others use or had experienced, but because many of his efforts were judged inappropriate, his deeper motivation of engagement with others was rarely responded to here. The pair with the ball consolidated their own sense of "we" by laughing. Corsaro (2003) describes children's attempts to exclude others from their interactions as "an attempt to keep control of their play, to keep sharing what they are already sharing (p. 64, italics in original). However, in this episode, their reaction seemed more aligned to positioning him as 'other' than as protection of their play. While the incident seems minor:

... such moments ... constitute swift lessons in the operation of power, mere glimpses of what friendship might entail, tiny gestures of disapproval or acclaim. This is how children come to understand their social world and it is this struggle for effective participation which makes the term 'socialization' seem woefully inadequate. (James, 2005, p.328)

If such positioning of Robert continued it would be likely to affect the way he defined himself within the centre as McCadden (1998) recognised. Robert T. was the clearest case of a child who was positioned as an outsider; other examples were typically situations where exclusion by one group was balanced by inclusion within another.

This discussion has suggested how important relationships were to children. Every child wanted to be an accepted participant (Corsaro, 1985; McCadden, 1998). Singer (1996) suggests children's “interest in one another and the value of these contacts are often underestimated" (p.36); these episodes suggested that the challenge involved in forming and sustaining peer relationships might be an underestimated area of curriculum. The primary focus of this discussion has been on children's role as friends, but these episodes have shown that issues of power and control were a frequent ingredient. While many relationships were richly satisfying, there was also potential for children to be rejected and dismissed by their peers. Finally, these observations were a reminder of how unique each child's curriculum experience was. Observations of Fleur throughout a day showed her path rarely intersected with James, which was a timely reminder of what might be glossed over in referring to children's collective curriculum experience. 


\subsubsection{Gender-defined roles}

This section describes how older children's interest in defining themselves by gender extended the boundaries of curriculum. It will be argued that the tension between being a participant within the community and exercising control was demonstrated most overtly in the older boys' superhero play, and in their personal interactions. Their activities also raised tensions around definitions of appropriate/inappropriate play which highlighted the teacher-child demarcation. It will be suggested that, for a range of reasons, the gendered role many girls adopted had less influence on the scope of curriculum.

The significance of gender in young children's peer groups is widely reported (Corsaro, 2003; Davies, 1989; Kyratzis, 2004; MacNaughton, 2000; Paley, 1984) and described in Chapter 3. Given others' descriptions of young children's vigorous appropriation of gendered roles (Blaise, 2005; Corsaro, 2003; Davies, 1989; Jordan, 2002; Martin, 1998; Paley, 1984) and their discussion of how these reflect wider social discourses (Davies, 1989; Jordan, 2002), the focus here is on noting what was atypical in this setting, and on showing how children's gender work demonstrated the themes of the thesis, and extended the scope of curriculum.

\section{Modelling yourself on the Action Man group}

The activities of a group of older boys, centred on James, provided a highly visible role model for others. On my first day observing:

\section{James rushed up to me and said urgently "Bad Jelly the witch is there. She's attacking. She can't get me because I'm in glass" staring at me urgently and holding a plastic spade which he had been wielding ... like a gun. [27/7/05]}

As well as being fast-paced and exciting, their play was often conspicuous because of their physical and verbal aggression towards each other, their threatening behaviour towards others, and their resistance to teachers. They were what Jordan (2002) calls "the fighting boys" (p.32) and Davies (1989) labels "the lords or superheroes of the playground" (p.122). The loose group around James included Walter, Aidy, Angus, Robert A., Rex, Levi and Alex. Of these only Walter, Robert A. and Levi attended for fewer than four days a week. Within the group Rex was an exception. He was the oldest boy in the centre, aligned himself with the group through his clothing, and often joined their play but was never observed using physical aggression. He was also unusual among the group in 
identifying a girl as a friend. His stance appeared to be accepted; he moved easily among groups and seemed universally liked. The example of Evie, discussed below, will show resisting gender roles was not always so easy.

Identification with Action Man figures was a strong theme in the play of these boys and in their own gender identification. They compared Action Man insignia on their clothing, and used Action Man references to define group membership.

James is inside again shouting "Batman". Aidy is there too and shouts "I'm Batman". Levi says "I'm Batman" and Aidy says "No you're not, you're not Batman". [9/8/05]

It seemed the personae were sufficient. No examples of the boys discussing indepth knowledge of Action Man figures were observed. When Rex's mother mentioned the Spiderman video he interrupted to say it was not a favourite.

The character of much of this group's play is demonstrated in the following episode, one of many similar observations. One morning James and Levi had been involved in a sequence of running-chasing-riding which had included a consistent thread of physical threatening and aggression. Aidy, Robert A. and Alex had repeatedly aligned themselves with the play, at times being absorbed and at times rejected. At one point, after all five had been riding circuits on the bikes, Aidy and Robert were standing with James and Levi, while Alex was some way off:

Levi and Robert A. begin what looks like a friendly tussle, with no clear cause. The boys yell "yeah". Aidy joins in and punches Robert hard. Alex [comes over and] also punches Robert. Robert is on the ground cryingand continues to cry for some minutes. A teacher comforts him and asks what happened. [22/8/05]

After being reprimanded, Alex and Aidy retreated to the fort. The teacher remained with Robert, comforting him and then sending him inside to have his face bathed. Aidy stood on the top platform of the fort, and holding his arm straight with his fingers pointed across the playground, made shooting noises.

Little Caitlin has approached the fort, and insists that a teacher support her. The teacher encourages her: "You'll be alright, you can go" but Aidy is standing with his legs askance on the stairs... The teacher says "Aidy, everyone's allowed". Caitlin goes past Aidy up the stairs to the top of the fort.

The teacher, standing below, asks Aidy who he is and he tells her he is Buzz Lightyear. He says: 
"I can kill you". And then a few moments later "I kill you—with my laser". Aidy makes a "di di di di di" sound, which is the sound that accompanies the weapon stance, pointing his arm.... The teacher encourages Caitlin to come down the slide, sitting down. She asks "How can [using her own name] talk to you if you kill her?"

This episode highlights the tension that existed around what constituted appropriate play. Such expression of power relationships in play is a frequently identified characteristic of boys' play (Jordan, 2002; Watson, 2005) but also recognised as an element of children's role play in general (Corsaro, 2003; Dockett \& Fleer, 1999; Lofdahl, 2002). Teachers supported the play with dress-ups such as a Spiderman costume, Batman masks and long capes and there was a Batman poster on the wall. They frequently allowed plastic shovels, rakes and sticks to be temporarily redefined as weapons, and on one occasion a teacher assisted in making rolled-paper swords. However, the fact teachers never participated in the superhero play suggests the ambivalence that Browne (2004) reports.

The frequent use of aggression within this play meant teachers repeatedly took a monitoring role, intervening and reprimanding. This acted to further embed the teacher-child demarcation, particularly in relation to this group of boys. This was not a role teachers sought; at times it seemed the boys' actions were intended to increase this sense of opposition. Aidy's threatening behaviour towards a younger female and his overtly aggressive language to the female teacher suggest an undercurrent of gender positioning within the expression of control/power that others have described (Browne, 2004; Keddie, 2003; Watson, 2005). The conflict that Jordan (2002) contends boys face between teacher-promoted values of nonviolence and media-derived images of masculinity linked to violence, power and autonomy seems pertinent here. It is likely the absence of adult males offering alternative models of masculinity in this setting, as in many centre settings, exacerbated the situation.

The episode also suggests Aidy understood there was a kudos associated with being aggressive, that it was a way of gaining approval within this subgroup of peers. It was striking that his apparently unprovoked physical attack was on Robert with whom he had largely been aligned through the earlier play. The physical force he used escalated the tussle to a different plane. It seemed those who were most peripheral to the play delivered the greatest physical aggression here. For teachers, such acts of physical aggression were entirely unacceptable, and they strove to channel the play of this group into more acceptable forms. 
Children were told "You need to play nicely OK?" but the reality for Aidy was that there were conflicting sources of approval and definitions of acceptability. I wrote "they are like moths to the flame" having seen how irresistibly Aidy and others were attracted to the meld of excitement, action and aggression James engendered. Their dilemma was that both definitions of what constituted appropriate play had attractions. Aidy struggled to find a balance between the two, between being a member of this boys' community, and/or of the teacher-approved community. Such interactions among these older boys underlined the depth of challenge they faced as they sought to mediate the complex intermix of conflicting impulses: the desire to establish their identities and to gain acceptance in multiple arena, and the impetus to exert some control in those arena, which needed to be balanced by an understanding of the multiple ways in which the exercising of control/power might assist or hinder the process.

These boys were influential models for others. This was partly because their play was conspicuous. In contrast to the girls' play it was faster, louder, took up more space, there was more verbal and physical aggression, and more teacher interventions (Fabes et al., 2004). These same qualities were also seen in their personal interactions with each other. The tension between the desire for relationships and the urge for control/power, and the ways in which they strove to mediate that tension were demonstrated most overtly in the interactions of this group.

Other children, and particularly younger boys, were tuned into this group. Twoyear-olds Thomas and Jordan, both with older brothers in the group, participated peripherally at times. Others observed from a distance and experimented with the roles. Anakin ( 1 year 8 months) outside in the playground heard one of the boys chanting "Da, di de da da". A second later, Anakin said "da da". I wrote:

He is clearly turned in to them-ignoring the two girls ... in his vicinity. Anakin comes up and sits beside me on the seat. Even here he shouts " $d a$, $d a " \cdot[4 / 10 / 05]$

Some younger boys seemed to feel a tension between attraction and intimidation. Cassidy hid behind me when Angus, wearing the dragon hood, came running to the fort.

When Angus has gone he says to me "I hate the monster, I hate the monster" in a serious voice.... A few moments later I hear Cassidy say "I'm the monster" and I am so surprised that I say "Did I hear you say that you were a monster?" and he looks at me with a little smile and nods his head. [17/8/05] 
Later observations suggested Cassidy was continuing to explore these roles; he referred to himself as Batman, and was particularly interested in the photograph of himself with a Batman face-paint. Identification with the Action Man figures was not restricted to boys. Amanda wore the Spiderman mask and Evie chose to have a Batman face-paint. Very occasionally girls were part of their fast-action episodes, but were more often aligned as the outsiders. When Aidy, Robert A. and Walter were role-playing tigers, roaring, and holding their hands threateningly:

Robert ... shouts out: "Watch out, I found some girls, they're here”.

(Aidy and Walter come out from under the fort) "They're here, over there. Get them, quick get them".

Aidy: "Rrrrrr".

Robert: "Get them". He throws a hoop at them, and then another. "Watch out, aaaarrrrh". [4/10/05]

While older boys seemed to be the source of physical aggression, observations showed how patterns of aggression rippled beyond this group. A much wider group of children were recipients of such actions, and some also used these strategies themselves. James stood intimidatingly in front of little Sunshine, Fleur pushed Sunshine away when she tried to reach into the box of trains, Jeff told me "Robert A. does lots of mean things to me", Robert T.'s mother suggested Alex would be an interesting child to study because he bites. Such examples made it clear that the older boys, through their consistent refusal to align their play with teachers' definition of what was appropriate, and through the way they interacted with each other, extended the boundaries of curriculum for all children in an unanticipated way.

\section{Pink is for girls}

A strongly stereotyped definition of girl-ness, was promoted most markedly by Grace and Maxine, two of the oldest girls. Defining oneself as female through wearing pink was as distinctive as the boys' display of action figures, and was conspicuous among the other older girls; Mooloo, Dora, Sina, Charlie, and Amanda all regularly wore pink. When Grace and Maxine dressed up in the centre, their choice was often glamorous: a bride, going to a ball, and they regularly requested Barbie face-paints. On their final day in the centre, Maxine dressed up in a purple velvet skirt, with a pink and purple scarf knotted around her bare chest:

Grace was even more gorgeous-the white and gold brocade long skirt, a golden sheer scarf tied around her top, and a golden apricot stiff sheer length of fabric held in a flowing mound on her head by the circlet of little white rosebuds. [1/9/05] 
Even in their day-to-day clothes they provided their peers with a visually more stereotyped female model than teachers did, who typically came dressed in practical clothes.

Many of Maxine and Grace's activities reflected a gendered role that revolved around cooking, and setting up private spaces which they filled with 'possessions'.

I see they have taken possession of the canvas tunnel, and have some things in there with them. As I walk past Grace is coming out saying "We need some more stuff" and Maxine rushes off saying "Be back in a flash". [8/8/05]

One afternoon they spent time in the sandpit making 'cakes' and talking about what Grace was bringing with her to Maxine's house. Later they moved inside and made 'muffins' at the dough table. However, the theme of 'being a girl' did not dominate their activities. On many occasions these were less gender-specific: they built a Duplo 'motor', rode fast on bikes, and dressed up as animals. When Grace drew what she liked doing at centre, it was a picture of the carpentry table.

While many of the themes of Maxine's and Grace's play were replicated in the activities of younger children, their play did not appear to be as influential a model as the older boys' play. There were several possible reasons for this. Their play was less conspicuous: it was less movement-oriented, they often played alone, and their play attracted less teacher intervention. Their play also 'fitted' the context better than the boys' in several ways. There were more resources that facilitated expression of their gendered identity: glamorous dresses, displayed images of Barbie (there were also images of women in non-traditional roles), facepaints, dolls and accessories for caring for them, and cooking equipment. Even the home-like aspects of the centre environment and the presence of seven female teachers supported their play themes. Teachers were comfortable and familiar with their play, and their approval was reflected in their easy engagement. A passing teacher asked what flavour their muffins were, saying her favourites were citrus. Finally, Grace and Maxine's influence as role models may have been diminished by the presence of Evie, who actively resisted the stereotyped gender role, and who attended every day. Maxine and Grace were present together on only two days each week and left during the research. 
Evie overtly resisted the stereotyped 'girl' role Maxine and Grace often promoted. When she put on a black dress she called herself Spiderman. When the teacher called her "surf girl" she replied "I'm surf boy". Evie's mother described her as "just a tomboy", adding that Evie liked blue rather than pink and resisted wearing skirts. Evie was the most enthusiastic participant in the house-frame building project, and one of the few girls who occasionally participated in the older boys' Action Man play, although it was typically in a peripheral role. She was one of the few older girls reprimanded for physical aggression, and the girl who most frequently resisted teachers. Often her aim seemed to be to draw attention to herself in a way more aligned to the boys' agenda. Sitting at a lunch table with a group of older boys:

Evie is crumbling her biscuits on to the table and then eating them. Evie turns so that she is sitting backwards on her chair, with one leg on either side of her seat. A few moments later she is crumbling her biscuit. She drops bits on the floor and then picks them up and eats them. She puts her feet on the table. She jumps up from her chair and opens the sleeproom door and looks in. [18/8/05]

Evie provided an alternative model for other girls. She was the girl whose behaviours most overtly indicated the tensions in mediating the dialectic of being an accepted member of the community and yet simultaneously exerting control/power. As with the older boys, her expressions of control/power sometimes appeared to be the route through which she sought approval and therefore relationships with her peers. Evie appeared uneasy in her role with both boys and girls at times, and seemed most comfortable in her interactions with the younger Cassidy. Towards the end of the research, it appeared she might be yielding to peer pressure when she came to the centre:

...dressed in pink skirt and striped pink and white t-shirt.... Charlie (also wearing a pink skirt and jersey) got Evie to stand up so she could see it. [1/11/05]

Sina was the only other girl who regularly resisted the gender model portrayed by Maxine and Grace:

Charlie [female] says "Barbie girl" and Sina says "I'm not Barbie girl, I'm Batman girl”. [15/8/05]

While the overt use of control/power was rarely evident in the older girls' interactions, the episode described earlier in which Maxine and Grace used their 'wings' to define themselves as 'other' reflects others' reports of girls' use of "more subtle methods for controlling one another" (Corsaro, 1997, p.154). It is noteworthy that the two girls Grace named as people she did not like to play with 
were Evie and Sina which echoes comments on the assured status of those who reflect the stereotype (Davies, 1989), and the dilemmas that may be experienced by those attempting to challenge gender roles (Davies, 1989; MacNaughton, 2004).

Children's interest in defining themselves by gender enlarged the scope of curriculum, and this was particularly so for the boys. Although there were also multitudes of observations of companionable cross-gender play, for some children, and particularly James, such interactions were much rarer.

\subsubsection{Children as peer teachers/learners}

With the description of this third role, children as peer learners/teachers, the focus shifts to children's role in introducing other aspects of curriculum. A range of indicative examples will be given, and these will be linked to the first theme, the adult-child demarcation and the separation from the 'real' world, because not all children's areas of knowledge were equally easily expressed within the centre. The final argument in this chapter returns to the opening discussion of teacher roles, and suggests that the invisibility to children of much of the educator role had implications for some children's view of themselves as learners in this setting.

There was a myriad of examples of children's peer learning/teaching. Sometimes a child took a momentarily expert role. Cassidy watched Mooloo drawing with five chalks in one hand and copied her.

David [10 months] discovers what a good sound the latch that holds the door back against the wall makes. He can also only just reach it which adds to the challenge of playing with it. ... in a moment it is Sunshine who is there having her turn at stretching up and handling it. [1/9/05]

Children had particular skills they demonstrated, taking a more consciously expert role. Maxine showed how she manoeuvred her body through a hole in the fort, and others tried to follow. Fleur knew how to wrap a doll, laying out the blanket carefully on the floor first; it was a skill she shared with Mulan.

More enduring examples related to areas of expertise which reflected out-of-centre interests. It was easier for some of these to be expressed than others. Children's interest in clothes was a constant and readily expressed interest. Discussions of food were supported by mealtimes, and particularly by the school-lunch routine when older children brought their own food, and by the weekly smorgasbord lunches for everyone. Interest in vehicles and 'work' was fed by watching activity on the nearby 
building site. Teachers arranged for police dogs to visit having seen the frequency of animal-themed play. Other areas of interest, however, sometimes highlighted the isolation of children from the real world (Brennan, 2005; Singer, 1996). Cassidy defined himself as interested in horses. The resources available to him in the centreplastic horses, horse books, a teacher-made book-helped him to share this area of expertise, but there was no access to a real horse. Aidy's interest in Thomas the Tank Engine was mentioned by his mother and by teachers, and was seen in his play with the trainset, and in his solitary dramatic play. Having the trainset and books present made it easier for him to share his knowledge with others. His interest in fishing was less frequently expressed. In the centre he fished with a stick from the fort, and carried a plastic fish in a container. It was only teacher questioning that elicited that his dad caught a kingfish, that the kingfish bit his finger, that the fish was too big for the trailer, that they cut it up, that it was hard get the knife through the skin, and that they ate it, even the eyeballs! [14/12/05].

There were new interests that arose. James's role as 'brother-to-be' caused a flurry of conversations among boys about having babies:

Aidy comes to me and tells me "I'm not a tiger any more. Cause I'm, cause I've got a baby. My mum didn't get a brother for me."

Researcher: "She just got a sister for you?"

Aidy: "No. I am going to have four babies, I'm going to have three babies."

Researcher: "Are you?"

Robert A.: “And I'm going to have three babies too." [4/10/05]

Having very young children in the centre gave them day-to-day experience of the reality of babies.

Some interests were less tangible but still influential. Macauley's passionate interest in exploring the environment was often a source of inspiration for others. After he had spent several minutes in engrossed exploration of a reclining baby bouncer, and began to lose interest:

David (who has been watching for some of the time) takes over the investigation. [22/8/05]

A few days later it was Sunshine who seemed inspired:

Macauley took three steps to great applause from the teachers-sitting at his level and clapping. ... Sunshine seemed to be inspired and stood up unsupported at more or less the same time. [30/8/05] 
As descriptions above showed, older boys were often role models for younger children. In a less obvious way younger children expanded the boundaries of curriculum for older children. They provided them with opportunities for expressions of nurturing and tenderness; this episode captures the gentleness that characterised many such interactions:

Macauley had just got up from his sleep and still had his dummy...in his mouth. He was sitting on the floor in his napkin and t-shirt. Sunshine...was sitting beside him. Walter was crouched down beside the two of them.

Sunshine was fascinated by his dummy and kept reaching out, and gently taking it out of his mouth. When she took it from him Walter would say "no" but quite gently, take it from her and put it back into his [Macauley's] mouth. Macauley was uncomplaining, but formed his mouth ready for it.

When it was not in, he took the opportunity to smile and verbalise a greeting to accompany his smile. [A teacher] intervened and said that she thought he could do without it now, and got Walter to hand it to her. Macauley roared in protest, so she handed it back to Walter who put it back into his mouth. [4/10/05]

Walter was never observed playing with dolls or soft toys, but babies were a source of continuing fascination and offered him a channel through which to express his tenderness.

Macauley's apparent fearlessness and physicality made him conspicuous amongst the younger children, and it was perhaps this that marked him out for particular attention from James. When Macauley's father picked him up at lunchtime one day:

James seems at a loose end. He wanders, and asks when Macauley will be back ... I realise there are no other big boys up. [24/8/05]

What was it that made Macauley a significant figure for James? Perhaps he felt a rapport with him, saw in his fearless exploration a reflection of his own persona as an Action Man? Perhaps it was his vulnerability which made possible a companionable relationship in which James's dominance was secure? Perhaps, as for Walter, Macauley provided him with an opportunity to safely express his gentle nurturing qualities?

Of course, not all interactions between Macauley and other children were peaceful. At times he interrupted their play, tried to take equipment they were using, or wanted to join in when he was not wanted. Even his impending approach could be seen as disruptive: 
The teacher warns Fleur “Macauley's coming to see you Fleur, to see your puzzle". Fleur cries momentarily as he tries to take a piece of her puzzle. The teacher says "Say 'It's Fleur's puzzle", and Fleur's cry turns to a laugh. [15/8/05]

Even occasions like these were, of course, learning opportunities because they gave children experience in finding socially acceptable ways of handling, or perhaps deflecting, his advances.

The presence of these younger children drew from the older children a more varied range of emotions and reactions than would have been elicited in a group of same-age peers. They offered opportunities for being responsible, being a carer and nurturer, being an authority, and being a teacher. Reflecting on the ways in which younger children enlarged the scope of curriculum helped me to recognise that every child, through their presence in the community, was a source of learning/teaching for others.

Learning the extent of Rex's interest in electronics from his mother underlined how easy it was for children's interests to remain hidden. Rex's mother identified his interest as "playing on the computer":

He can turn on the computer, knows to wait for the egg timer to go away, use the mouse, link on to the Internet, select favourites, maximise and minimise, shift the tool bars around. [2/11/05]

Rex showed aptitude in using the digital camera introduced for the research, but at that time there was no other electronic equipment accessible in the centre so there were limited opportunities for him to share his expertise with his peers. (A teacher reported she occasionally brought in her laptop for him to use, and a digital camera was later purchased.) Knowing it was only this conversation that drew my attention to Rex's interest underlined the significance of teacher-parent communication in identifying children's funds of knowledge (Hedges, 2007), and served as a reminder of how many other interests I might not identify.

Analysis of children's role as peer learners/teachers showed it was an expression of the adult-child demarcation. Because the centre was an isolated environment, apart from the real world, not all interests were equally easily shared. Sharing occurred most readily when resources supported their expression and when teachers acknowledged the interest. However, the many ways in which peer teaching/learning was occurring suggests potential for future blurring of the teacher-child demarcation; this will be discussed in Chapter 9. Because the focus has been on content rather than 
relationships through this discussion, the linked curriculum concerns of establishing relationships and exercising control/power within those relationships that were introduced as the second theme of the thesis have been less evident.

\subsubsection{Do children see themselves as learners?}

One final theme arising from data analysis of children's role as learners/teachers related to the older children's perceptions of themselves as learners. Analysis suggested that, for a range of reasons which related primarily to the teacher-child demarcation around the role of teacher as documenter/educator which was outlined in the first section, older children did not readily identify themselves as learners in this setting. The analysis that led to this conclusion has been detailed elsewhere (Stephenson, 2008, 2009). In summary, three lines of analysis contributed to this conclusion. The first was the infrequency with which the language or the concepts of learning and teaching were used in teacher-child interactions although this language was a feature of all teachers' planning documentation. Secondly, conversations with children and their responses to the New Kid book (Research strategy 6) suggested they did not readily identify what they or others were learning in the centre, or what teachers might teach, although it was clear some saw themselves as learners in other contexts. Thirdly, parents of older children who were interviewed found it harder to identify what their child might have learnt than parents of younger children.

The conclusion that older children seemed not to identify themselves as learners in the centre was challenging, but not unanticipated (Carr, 1997b) and followed logically from the invisibility of many aspects of the teacher as educator role, outlined in section 6.1.5. A consequence of this was the limited opportunity for teacher-child partnerships around children's learning. Identifying a link between older children not seeing themselves as learners in the centre, and the visibility/invisibility of the teacher as educator role was a finding unanticipated in the literature. This finding has a raft of implications for how and when the discourse of learning/teaching is used and who uses it, for children's role in discussions of learning and teaching, and perhaps also for the kind of experiences offered for older children within early childhood education. These lie beyond the scope of this thesis. 


\subsection{Conclusion}

The descriptions of children's roles as friends, as boys/girls and as learners/teachers, have identified an indicative range of peer-generated curriculum content which expanded the boundaries of curriculum in the setting. It seems likely that in our professional focus on the teaching role we may be in danger of ignoring these other layers of children's curriculum experience, and of underestimating the significance they have for children.

The second emergent theme was introduced in this chapter; this recognised that a predominant curriculum concern for children was establishing oneself within the community, with a subsidiary, but interweaving thread related to the exercising of control/power. These dual concerns were seen within children's role as friends and their gender-defined roles. Discussion of children's role as peer learners/teachers focused on curriculum content they introduced. The first theme, the adult-child demarcation as a source of curriculum boundaries, reappeared in the discussion on teachers' roles, and was identified as a factor that contributed to some children not perceiving themselves as learners in this setting. In Chapter 7, the third and final sensitising concept, the routines/rules/rituals, is used as the framework for further exploration of these two emergent themes. 


\section{CHAPTER 7}

\section{RULES, ROUTINES AND RITUALS}

I hold that orthodoxy is the death of knowledge, since the growth of knowledge depends entirely on the existence of disagreement. (Popper, 1994, p.34)

In this chapter the third concept from activity theory, the rules or norms, is used as the lens through which to examine the data. In considering how the norms or rules might impact on children's curriculum experience, it was decided to focus first on children's experience of centre rules, and then on their experience of some centre routines and rituals. Distinguishing between routines and rituals was not selfevident; for example, some routines contained ritual-like elements. Rather it seemed they lay on a continuum. However, mat-time, mealtimes and sleeptime were selected as typical routines, and the celebration of a fifth birthday was chosen as an example of a ritual.

In the first section it is argued that adult rules revealed adult-defined curriculum boundaries, but that children's resistance to rules both enlarged the scope of curriculum, and reinforced the status of power as itself an element of curriculum. An unanticipated finding here was that teachers and children used moments of resistance as opportunities for negotiation at times.

The content of the second and third sections act to consolidate the arguments put forward in the previous two chapters. They show how the two emergent themes of the thesis - the adult-child demarcation as a source of curriculum boundaries, and the dual focus on establishing relationships and exercising control/power as aspects of curriculum - reappeared in a variety of guises when this third perspective, the rules, was applied to the data. The research strategy the 'Naughty book' (Research strategy 7) is introduced in this chapter; see Chapter 4 section 4.4.3 for a description.

\subsection{Rules}

Children's experience of, and reaction to, rules and the influence this had on curriculum boundaries is the first area of analysis in this chapter. Rules were a mechanism through which teachers imposed parameters on children's curriculum experience and children, through their reaction to these centre rules, influenced 
those parameters. Therefore this analysis yet again reinforces what has been a continuing theme through the last two chapters, that the adult-child demarcation was a central source of curriculum boundaries.

An element within the second theme of the thesis is that issues of control/power are curriculum concerns for children. This line of reasoning is reinforced by descriptions of children's resistance to teacher-derived rules in this section. Children's experience showed there was variation in rule enforcement and this influenced the scope of children's resistance. Some children also used and developed their own rules as a way of exercising control/power in peer interactions; however, these incidents were found to have less impact on the boundaries of curriculum, than children's reaction to teachers' rules.

Focusing on teacher-derived rules is like stripping off the skin of community and seeing the bones of power exposed. This is the formal organisational structure that defines curriculum and holds the community together. The adult-child demarcation is clear; the vast majority of rules originate from teachers, and are underpinned by assumptions that adult rules are inherently 'right' and children's reasons for rule-breaking are inherently misguided (Waksler, 1991). For some rules, based on government regulation, teachers were enactors rather than initiators, but to children all rules appeared to be teacher-derived. Perhaps only for Angus, who was aware that teachers were complying with his parents' request that he have an afternoon sleep, was this not true.

Children understood there were rules. Mooloo explained the cones on the concrete were to stop the bikes. She described other centre rules as:

"No biting", and then elaborated "No fighting and no biting". When I asked if there were others she said "That's all". [29/7/05]

The unfinished storybook about the 'naughty child' (Research strategy 7) was devised to explore children's ideas about what was inappropriate centre behaviour. However, children's suggestions were limited: "smashed the toys", "throwing toys", "throwing everything", "fight everybody and punch everybody", "fight her brothers" and be "cheeky" [17/10/05, 20/10/05]. Perhaps the word "naughty", not one used by teachers, was confusing. The suggested ideas and the reference to fighting brothers seemed more indicative of what might be defined as 'naughty' at home. 
There was no list of centre rules. Most teacher-derived rules were inferred from observation of what others did, and from deduction, working primarily from episodes of infringement (Waksler, 1991). Rules were spoken when they were broken, or when there was a likelihood of infringement. (If compliance had been complete, there would apparently have been no rules.) The only observed examples of groups being formally 'taught' rules in the way others report (King, 1978; McCadden, 1998) were two teacher-facilitated mat-time discussions about what hands should be used for, and how school children sit, although planning documentation also recorded a discussion about rules. The infrequency of such sessions is likely to reflect the less formal programme, children's consequent greater freedom, and the higher adult:child ratio, which all contributed to less need for overt regulation. However, the fact that children were reminded of more than 60 guidelines and rules during observations attested to the high level of adult control embedded within the apparent freedom (Cannella, 1997). Alcock's (2005) comment, in relation to similar New Zealand settings, that children "were subject to a lot of control and surveillance" (p.244) suggests this may not be unusual.

These rules, the majority of which would be familiar in other centres, were aimed at ensuring children were safe, the programme operated smoothly, and relationships were harmonious. Collectively these rules set down expectations of curriculum boundaries. As discussion in Chapter 5 showed, there were rules covering the use of the environment ('No playing in the locker room') and resources ('Dough stays on the dough table'), the management of routines ('Put your cup on the trolley'), and personal possessions ('Wear your sunhat outside'). Rules relating to how children interacted with each other ranged from prohibitions of 'No interfering with others' work', and 'No touching others' (during mat-time) to the exhortations 'You need to take turns', 'You need to share'. Teachers also used broad injunctions. 'Sit up nicely' was easily understood because there were visible models. 'Make good decisions', 'Make wise decisions' and 'Play nicely' were less obvious and raised the question of whose definition held sway. The frequency with which teachers invoked rules with some groups and in some contexts underlined the fundamental tension between maintaining the harmonious community, yet enforcing rules when that harmony was threatened.

Children's experiences showed rules ranged from permanent to temporary, from immutable to variable, and from non-negotiable, to open-to-negotiation. There 
was also variation in how reminders/reprimands were delivered and in the consequences for infringement. Such variations encouraged children to use interactions around some rules as a site for expression of resistance to teachers. This variation largely reflected the source/s on which a rule was founded (Waksler, 1991). The more a rule drew on cultural and social norms the more immutable it was, and often the more covert its expression. For example, it was an unspoken rule children did not urinate outside. Had any child done so, this rule would have been invoked. Rules that were more strongly underpinned by social and cultural norms, or by regulation, were more consistently enforced. When a child put a foot on the table during mealtimes, they were invariably told to put it down, whereas children who forgot to put their mug on the trolley were not always called back. Children's experience of rules as variable was heightened by the fact teachers sometimes developed temporary rules. At a staff meeting a teacher explained she made a rule 'No play fighting' after children wrestled on the gym mats; "If you do it again—inside and time out and no warnings" [4/10/05].

For children, a teacher's personal values were the wild card (see Figure 9). These might comfortably reflect a centre rule, favour strict enforcement of that rule, or be in tension with it. If a teacher's personal values reflected or reinforced a rule, the child was less likely to experience the rule as negotiable. For example, while children were expected to be polite, some teachers put more emphasis on children saying please. When there was a tension between a teacher's personal values and a centre rule, children were more likely to experience the rule as variable. For example, when a child was found to have a possession from home, some teachers complied with the rule and requisitioned it while others advised the child to keep it safe. 


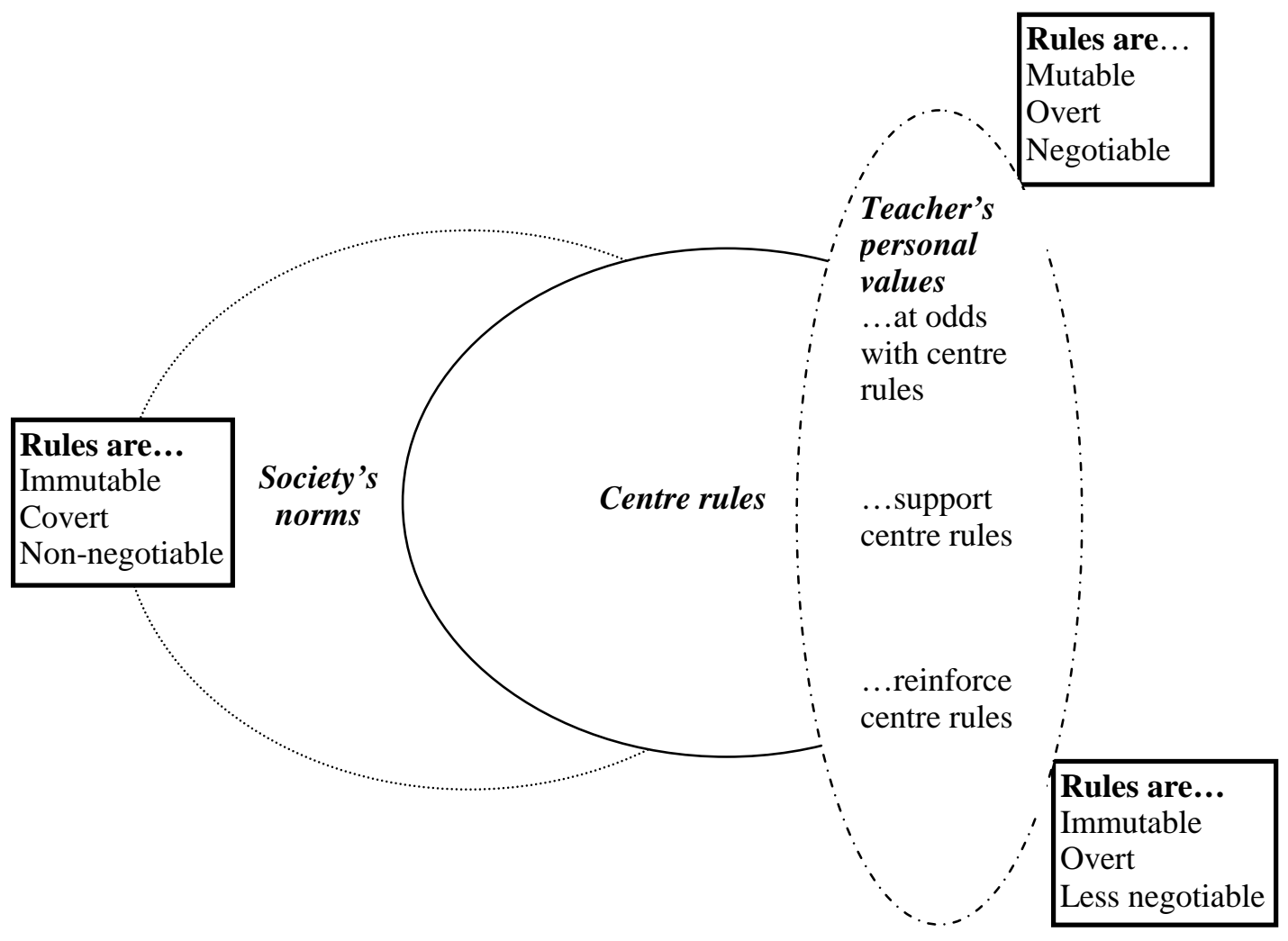

Figure 9: Factors contributing to variation in how children experienced rules

Variability in enforcing rules encouraged children's sense of agency (McCadden, 1998). Through testing rules, children developed their own personal response to them, and began to comprehend the existence of a range of varying interpretations within the context. However, for a novice like Robert T., such flexibility potentially added to confusion about what was permissible. Even when a rule was expressed as immutable and non-negotiable by teachers, children were sometimes still prepared to challenge it. This was conspicuously the case for some older boys. Their continued bouts of aggression, in the face of teachers' united and sustained disapproval, indicated the significance that issues of control/power had for them. However, it also suggested a fragility to teachers' control. When one of the teachers apparently jokingly commented in a staff meeting "I'm frightened of James", it seemed that the balance of power underpinning the adult-child demarcation might not always feel secure.

\subsubsection{Children's resistance to rules}

Resistance is a central concept in the writings of critical pedagogues, where counter hegemony is recognised as the resistance to and struggle against hegemonic control, and is therefore the potential source for change within the 
system (Kanpol, 1999). In his work with Brazilian peasants, Freire encouraged them to develop clearer antagonisms between themselves and the dominant group as part of a process of developing their own identity (Janmohamed, 1994). In this setting it will be argued that children at times emphasised the adult-child demarcation for their own purposes of gaining acceptance and status among their peers.

A variety of explanations have been offered for young children's acts of resistance within early childhood settings. Firstly, they have been seen as a reaction to adults' power (Cobb et al., 2005). Corsaro (1997) saw “children's desire to achieve autonomy from the rules and authority of adult caretakers and to gain control over their lives" (p.131) as a major theme of peer culture. The argument presented here confirms and extends that explanation, by showing how children did gain some level of control through resistance.

An allied explanation is that resisting adults' rules is a mechanism for strengthening group identity (Alcock, 2005, 2007; Brennan, 2005, 2007; Corsaro, 1985, 1997 , 2003; Kyratzis, 2004; McCadden, 1998). In a New Zealand setting Alcock (2007) found children's playing around with rules at mealtimes was a way they created a sense of "togetherness" (p.286). In the centre, there were demonstrations of resistance that reflected this sense of togetherness; for example, some older children's shared resistance to coming inside for mat-time bordered on becoming a ritualised example of resistance. However, it is argued that the reference group was typically a subgroup of peers rather than the whole peer group.

There are also explanations which focus on the broader social purpose. Alcock (2005) also argues children's playful subversion of rules, their testing and their innovating, are ways in which they are developing their understanding of rules and rule-breaking: "children actively appropriate, re-create, and learn rules for living through ongoing processes of internalisation and externalisation of the rules of their cultures" (p. 243). Brennan (2007) expresses more uncertainty about the motivation for children's resistance, but suggests “children's repeated transgressions signal to adults that the "fit" between cultural expectation and cultural reality may need realignment" (Brennan, 2007, p.4). While observations in the centre confirmed such a process of adaptation occurred, the focus of the argument developed here is on the child's role in that process. 
Teachers' programme planning documentation showed they understood and accepted children would experiment with resistance to rules:

Levi is developing a greater understanding of the limits/rules at centre and how far he can challenge these! [Progpl12]

Teachers typically responded positively, and sensitively, to children's resistance.

The youngest children's negotiations were often non-verbal:

When I arrive Anakin is just up from a sleep, and the teacher is taking her time getting him changed. Telling him before each thing she does, and letting him slowly readjust to the world. He babbles "mummy" slightly disconsolately.

She confers with him about his clothes. He points to a football shirt in his bag to put on, but she shows him it is wet-lets him feel it—and then asks if he wants the white jersey. ... When he alternatively shakes his head, and then points to it, she puts it over his head, to gauge what his reaction is. [27/9/05]

When children were divided into groups for mat-time, Fleur lay on the floor crying, apparently because she was separated from her favourite teacher, who then invited Fleur to join her in "the 4-year-old mat time". With older children the process of negotiation was more often verbal:

The teacher asks "Cassidy, would you like me to help you put your shoes on now, or would you just like to have morning tea in your socks? What would you like to do?"

He says "Have morning tea in socks"

She says "Ok, but remember if you go to play outside you need to put your shoes on" [26/9/05]

Some children rarely, if ever, overtly resisted teachers. As McCadden (1998) found, girls were more likely to accommodate to the rules. There were observations of minor or no resistance for 12 girls and 5 boys, but their names are not the ones dominating these pages. Again as McCadden found, it is the resisters who hold the limelight in the centre. And Anakin (1 year 8 months) and Emjay (1 year 5 months), who increasingly gained confidence in joint displays of overt resistance, served as a reminder that children's patterns of behaviour will change over time. As described in 6.3.2, Anakin's echoing of the older boys suggested how tuned in he was to them.

Aidy comes up to me, and roars, and uses 'claw' hands towards me.

He tells me he is a "tiger" and "goes off roaring".

Close by Anakin continues to echo his roars. [4/10/05] 
I note: He knows what you do to be a big boy.

Later, when James was sent from mat-time to sit on a chair, he put a box on his foot like a shoe:

Towards the end of mat-time, Anakin and Emjay got up and wandered over there. A teacher called their names—she was holding [a baby]—but they did not show any sign of responding. They wandered on past James, and around to the storage box for collage, where Anakin picked out a cardboard box, similar in size to James's.[4/10/05]

A teacher brought them back to the mat. Two months later their shared act of resistance at mat-time was more pronounced:

Emjay and Anakin are sitting close to each other and both lie on the floor on their fronts, and in sing-song voices chant "ahah ah ah aha ah ah". It is not clear who is starting but when one starts, the other joins in immediately. They do this with pauses, resisting "Sit down please", "Ankin and Emjay", "Shhhh", an adult moving over to sit beside them, a warning that they need to be quiet because the babies are asleep.[16/12/05]

Eventually they were led away to wash their hands for lunch.

Analysis of children's acts of resistance to teacher-imposed rules led back to the two emergent themes of the thesis. While it was argued in Chapter 5 that the adult-child demarcation was a central source of curriculum boundaries, here it is shown children at times made use of that demarcation for their own purposes. By identifying teachers as the ones-to-be-resisted, children reaffirmed their membership of the peer group, as others (Alcock, 2005, 2007; Brennan, 2005; Corsaro, 1985, 1997, 2003; Kyratzis, 2004; McCadden, 1998) have shown. However, in this setting it was found the reference group was typically a subgroup with which the child identified. Collective acts of resistance appeared to be a way of consolidating the sense of 'we-ness' within that group. Individual acts of resistance to teacher/s, in the presence of others, often seemed to be aimed at gaining/maintaining status within the subgroup. At times it was clear approval from the subgroup was of more immediate significance than teacher approval. This may be because children never appeared to doubt teachers' long-term acceptance of them. Some children, however, seemed to find it necessary to constantly reaffirm their acceptance and status among their peers.

Children demonstrated resistance in a variety of ways, as Brennan (2005) also found in a similar New Zealand setting. Most frequently, it was observed in their actions: disregarding teachers' requests, delaying compliance and/or continuing 
the infringement. As Corsaro (2003) also found, children's resistance to tidying was an everday example:

There is a call for tidy up time in a child's voice. The teacher says "Tidy up time" and then shouts "Everybody put your bikes up on the grass please, by the fence". None of the [older] boys respond. [7/10/05]

When another teacher called them to mat-time, they abandoned the bikes and went inside, using the second request as an excuse to ignore the first. Leaving an area was not always sufficient.

Robert T. tips the whole crate of Duplo out onto the mat, and then wanders outside. Evie is sent by a teacher to bring him back. [26/10/05]

The most unexpected example was demonstrated by Caitlin, aged 20 months, with food at the end of mealtimes.

Teacher: "Caitlin, you still got it in your mouth?"

Three seconds later, Teacher: "You still got it in your mouth? Have a little drink of water, ok?" which Caitlin did and then sat there with her cheeks bulging.

Thirty seconds later the teacher said: "Caitlin, swallow it" in a warning voice, but Caitlin just sat there.

Ten seconds: "Caitlin, swallow it".

An adult said: "She always does this". [12/12/05]

I wrote: An interesting way of exercising power.

Children also expressed resistance verbally. When a teacher asked Aidy, balancing on a shelf to reach the CD player, to get down:

He said "No, I won't" and she ... shifted the box on the shelf he was on, and commented on making it safe. [27/7/05]

Very occasionally a child responded to a reminder/reprimand with a direct verbal attack. When a teacher attempted to negotiate an alternative site for the crates:

He says "I don't like you".

Teacher: "I don't mind if you don't like me. You can't leave them there". [9/8/05]

Children's resistance was often the public display, in front of peers, that others describe (Alcock, 2007; Brennan, 2005; Corsaro, 1997).

Jordan had brought two plastic pigs with him to the table, and Alex had one, which he put into his cup. The teacher told him to take it out, "dirty" and he ignored her-and she told him he shouldn't drink it, and he did. [25/8/05] 
The only episode of resistance which united all children occurred during lunch. While teachers were busy James turned the lights off and, with Sina's help, pulled the curtains making the room interestingly dim. A few moments later teachers pulled them back, and turned the lights on, without comment. James and Sina repeated their actions. The curtains stayed shut but a teacher switched on the light. Other children joined in, and every time a teacher turned it on, a child would find a chance to run and switch it off. The light went on and off ten times. The changes in lighting meant everyone was aware and watching with increasing suspense as the interaction continued. Finally, a teacher intervened, explained the fuse would wear out, and stationed herself by the switch. For a few hushed moments, however, these acts of resistance had been the collective focus for all children, and allowed many to experience the thrill of transgression, without jeopardising their own acceptance within the teacher-child community.

It was clear children learned through watching peers:

Evie is at the door to the deck-she shuts it so Robert T can't come in, and holds it shut. A teacher opens it. Robert comes in-he then shuts the door so that Angus and Rex and the teacher are still outside. [18/8/05]

Fleur mirrored the older boys' resistance to the mat-time call, waving goodbye to the teacher from the top of the slide.

\subsubsection{What might resistance achieve?}

There often seemed to be a complex mix of potential motivation for children's acts of resistance. When such resistance was collective, the significance of the expression of control/power directed at adults seemed to be a strategy of relationship-building amongst peers. Friends followed each other:

James puts his socked feet up on the table. The teacher sees and says
"Please keep you feet under the table". Rex briefly puts his Spiderman
gumboot on the table, but takes it down before anyone notices. [18/8/05]

There were episodes which suggested knowing actions were disapproved added piquancy to the delight in a shared activity. Evie had been lifting up one side of the water trough, but was told:

“Evie, stop it, that's going to hurt somebody's toes".

When the teacher moved away, Evie and Robert A. returned to the task:

Evie: "We're going lifting this up eh? By ourselves".

Robert: "Whaaa, ooooh-I've got it, I've got it". 
They both laughed:

Evie: "Tip it out shall we?"

Robert: "Yeah".

They lifted the side again causing water to slurp, and giggled.

Robert: "Do it again, do it again". [22/8/05]

Here the act of bravado in resisting a teacher was exciting, but it was also a way of creating an alliance and perhaps gaining approval from peers. Evie and Robert, collaborating together, shared a moment of close companionship.

As indicated in Chapter 6, some episodes of boys' play suggested expressions of male dominance towards both younger female peers and female teachers, were elements within acts of resistance:

James stands in front of Sunshine who is on the mat-his legs askance in a menacing stance. He gazes at her and says "No" and moves closer. The teacher says from the table "You leave Sunshine alone please". James moves straight back to menace her again, his foot very close to her legs. [9/8/05]

While other scholars tend to emphasise the significance of resistance for children's sense of togetherness, and as an element of shared peer culture, evidence here suggested resistance was also important as a way in which children were experimenting with exercising control/power within an adult-dominated world. Here power was, as Foucault described, exercised and interwoven into relationships (McLaren, 2007). Individual acts of resistance, private interactions between a teacher and child, provided the clearest examples of children experimenting with the exercising of power/control with adults. With no peers present, it was clear the motivation was not about enhancing peer status. Often it seemed the immediate impetus was a child's level of involvement. Aidy's determination to reach the $\mathrm{CD}$ player, as he balanced on the shelf, was such an example. Another was Thomas's reluctance to leave the trainset and wash his hands. The teacher put his linked engines up high but:

... he still does not leave, and is carrying Bertie the Bus. She suggests he puts it in his pocket to keep it safe through morning tea. [19/10/05]

It seemed that inherent, usually implicitly, within such acts of resistance was a call for negotiation. In the example of Thomas it was signalled by his silent refusal to comply. The teacher's suggested compromise met his implicit request to negotiate, without relinquishing her broader aim of gathering everyone for 
morning tea. When Robert A. did not want to take off the Spiderman suit to sleep, he was allowed to keep it beside him. Occasionally children explicitly negotiated, or combined explicit and implicit negotiation. When Rex was asked to put a paper ball in his pocket, he explained he did not have one. The teacher told him to put it up his sleeve:

He does but takes it out again, and asks if he can put it on the chair. [12/10/05]

In such episodes the teacher still held the balance of power, usually proposed the compromise, and held the right to grant/refuse, nevertheless children learned resistance gave them some control, and that the potential for negotiation existed.

Such moments of individual resistance are not often described by others. Indeed Brennan (2005) writes "lone expressions of independence and resistance were meaningless empty acts without others to witness and sanction transgressions" (p.207). Here, I would argue, lone expressions of resistance were in fact moments in which children explored how they might exercise control/power in interactions with teachers, and as the examples showed, such resistance often achieved a measure of success. A compromise might be immediate, or it might be won as the result of repeated resistance, as the example of Angus and the requirement that he sleep will show. There his repeated physical demonstrations of resistance led teachers to change the requirement. From Angus's perspective this was an acknowledgement that he had some control/power within the situation; for the system as a whole it exemplified the process of cultural adaptation in the light of resistance that Brennan (2007) identifies. Within the context of this thesis, it is an example of teachers finding ways to mediate the dialectic tension between relating empathetically to children and exercising control over them.

For a child, achieving an immediate compromise was likely to contribute to their sense of empowerment and could strengthen the teacher-child relationship. More importantly for this thesis, such moments contributed to the blurring of the adultchild demarcation which underpinned the rules. This is in contrast to the teacher reaction to resistance described by McCadden (1998): "she did not see this challenge as an opportunity to develop negotiation skills in all the children, but rather saw their actions as manipulation on their part and therefore a threat, something to be quelled" (McCadden, 1998, p.66, italics in original). However, although centre teachers appeared more sympathetic to children's perspectives, 
they also did not often use resistance as an opportunity for verbal negotiation. Yet research by Singer and Hännikäinen (2002) indicates the effectiveness of teachers' use of child-centred mediating strategies to de-escalate children's conflicts, and they encourage greater use of negotiation. Interestingly centre children were encouraged to, and did, use negotiation skills in peer interactions. James approached Charlie and asked:

"Please can I have these?" He and the other boys have carried all the other crates up to the high platform on the fort. She says "No" very firmly. He says "We don't have enough". [8/8/05]

The most sustained adult-child negotiations heard were the interchanges between Rex and his mother about when she could leave. These examples indicate the potential for more verbal negotiation between teachers and children, as a way of supporting children in their experiments with the exercise of control/power, and of further moving towards Cannella's (1997) vision of children as emancipatory agents with voice in their own lives.

Reflecting on children's experiences of rule-making and rule-breaking has shown how children made use of the power relationships embedded in the adult-child demarcation. As children used their agency to test the boundaries, so they were also becoming knowledgeable about the exercising of power. Brandtzæg (2006) suggests children's relative powerlessness leads to the covert quality of children's resistance. This thesis offers an image of children as more robustly proactive, and shows children's resistance and challenge was a consistent and visible dimension of centre life. Finally, the experiences of rule-making and breaking also reiterated the complexity of judgements teachers must constantly make: "The action is too quick, the decisions have to be made too fast, and the situations never seem to fit theory or prior experience precisely" (McCadden, 1998, p.5).

\subsubsection{Children's own rules}

Three types of child-derived rules were observed. The first were rules created in the moment to protect ongoing play that others have identified (Corsaro, 2003; McCadden, 1998). Corsaro (2003) suggests excluding others is not a refusal to share but rather an attempt to protect the ongoing play. When Cassidy and Fleur were inside a circular enclosure laughing delightedly as they bounced a large ball, Cassidy says: 
"No, you can't get in" firmly and loudly when Anakin begins to climb in. [25/8/05]

Here the motivation seemed to be protecting their shared moments of mutual enjoyment. In other examples, a stronger element of 'power over' appeared within the exclusion. When Robert T. was attempting to retrieve a train that Thomas had taken from him:

James steps across and says very loudly "Robert, don't play with Thomas's
things" and stares at him. Angu steps across and bends down and goes
"Grrr" in Robert's face. James says "You're very naughty". [26/10/05]

The episode when Aidy blocked Caitlin's access to the fort (discussed in Chapter 5 ) is a similar example.

The second type of child-derived rules, were rules some children presented as teacher-derived. Although Jordan et al. (1995), in an Australian study, found children invoked adult rules with unexpected frequency, in this setting only four children used teacher-derived rules to exercise power/control over others, usually by drawing teachers' attention to transgressions:

James shouts out to the teacher "He [Angus] has gone through the cones". [18/8/05]

The same four children also used rules that sounded as though they were teacherdefined, yet were never heard enforced by teachers. When Mooloo was at the art table using the glue and brush, she announced:

"Babies aren't allowed to glue", but later the teacher says that they are only not allowed to eat the glue. [8/8/05]

Because Caitlin's gluing had distracted me, it seemed likely Mooloo's motive was to regain my attention. When Alex approached a group drawing with chalks on the concrete, took the chalk container from Emjay saying "Only one", then emptied them all out, the motive was less clear. Was it a strategy to draw attention to himself? A request to be included? Or was it expression of power in a social context where he assessed there would be no retribution?

The third type of child-derived rule was the setting of temporary rules within a play interaction. Usually there seemed to be no retribution for occasional infringements. When Maxine and Alex were playing a follow-the-leader game she instructed him: 
"You go this way", "Follow me" but while he largely followed her, he often took a short cut in the circuit, and came up somewhat in her way. [29/7/05]

Occasionally there was a greater element of power-over in a child's rule-making within a play interaction. When Walter and James parked their trucks alongside each other:

Walter ... approaches James and says "Hey mate, how are you?" He and James repeat this to each other, laughing. There are two tyres ... and Walter goes to pick up a tyre. James puts his foot on it, and says "No, no" loudly and firmly, angrily. Walter says "They're not so heavy". James says "It stays here" (loudly) and his foot is still on it. [18/8/05]

Here the shared moments of bonhomie are interwoven with James's struggle to control Walter's actions, yet again demonstrating the tightrope children walked at times, balancing the desire for companionship with the desire for control.

This discussion of rules has shown their influence, but has also highlighted the ways in which children resisted those boundaries, and through this resistance influenced the boundaries of curriculum. In particular it reaffirmed the importance of power as a curriculum concern for children. This discussion has also confirmed others' descriptions of resistance as a significant element in peer culture, but has extended and elaborated those descriptions.

\subsection{The centre routines}

"Routines" has two overlapping meanings. One is "the habitual way things are done" and the other, a use particular to early childhood education, refers to the regular events of arrival/departure times, mat-times, mealtimes, sleeping, toileting and tidying. This latter meaning was adopted here because of its greater relevance to the profession, and because it increased opportunities to build connections with other recent New Zealand studies (Alcock, 2005; Brennan, 2005). Because tidying has been well described (Corsaro, 1985, 2003) and similar patterns of resistance were observed here, and because toileting and arrivals/departures were not group events, it was decided to focus on mat-times, mealtimes, and the post-lunch sleeptime. While the adult-child demarcation was inherent in all three routines, the argument here focuses on the second theme of the thesis, the significance of relationships with others, and the exercising of control over others as aspects of curriculum. Mealtimes were important for peer relationships; mat-times were significant as the forum for teachers' building of community and shared culture, but 
could also be occasions of high teacher control and high child resistance; sleeptime was the site of both intimacy and conflict between children and teachers.

\subsubsection{Mealtimes}

Mealtimes were, par excellence, a time for peer relationships. Older children sat at tables, with the highchair children between them. Children usually chose their own seat, although occasionally teachers used nametags to define positions. At morning and afternoon teas a child was given responsibility for sharing the plate of fruit and toast/sandwiches at each table. Lunchtime began with children and teachers chanting the karakia (Māori grace) before a hot meal was served. Special versions of lunch occurred twice a week. One was the popular 'fun lunch' when children served themselves from a smorgasbord of finger-food, the other was 'school lunch' when four-year-olds brought a packed lunch from home and ate together.

Mealtimes were relaxed affairs for children. While teachers had clear expectations about behaviours, because they were busy, minor infringements often went unnoticed and the cheerful noise level meant teacher interventions were less obvious than at mat-time. Teachers coaxed but never required children to eat. Children were positive about mealtimes. In identifying liked/disliked activities (Research strategy 3), school lunch was a popular choice with older children, but was also chosen by younger children who did not participate in it. (There was no photograph of fun lunch).

Mealtimes were a chance for children to extend relationships with peers. While older children often tried to organise who they sat with, younger children appeared less concerned. At the most fundamental level, sitting in small groups allowed children to become acquainted with others. For new children, like Ruby, this was important, but even Evie, who had been coming to the centre for years, paused as she named the children at her table when she reached Jonathan, who only came twice a week.

It seemed that either she did not know Jonathan's name-or it certainly was not at the tip of her tongue. [30/8/05]

Realising how often children did not know each other's names continued to surprise. 
For a younger child, sitting with older peers offered a chance to be an audience, and perhaps participant in interactions. Sometimes these were moments of shared playfulness. A group of boys chuffing in unison were reminded:

"Excuse me at this train station. It is a bit noisy. Pretend you are coming into the station." [19/8/05]

The school-lunch children, sitting in a circle on the mat without a teacher, piled their hands in the middle, then peeled them off in sequence. As Alcock (2007) describes, mealtimes were also a site for playing with words and meanings. Younger peers overheard good-humoured ribald interchanges: "You're a bum, you're a bum" "Shooky lala, shooky lala". They watched how their peers captured attention with their bodies. Rex and Alex demonstrated burps, Sina pulled her eyes slanting, Angus draped his body across the rubbish bin. Occasionally younger children were the initiators. When Macauley began banging his mug, other highchair children joined in and, for a moment, there was shared delight as they banged mugs more or less in unison, before the teacher gently intervened.

Mealtimes were an opportunity for children to introduce topics, with less verbally adept children listening and occasionally contributing. Often it was the food itself; Fleur watched as Aidy ate the sausage skins he called "scraps", and they compared how many sausages each ate. Mooloo reproved Jonathan for not eating crusts. Rex looked in Caitlin's mouth when she refused to swallow. School lunches were a rich source of interest for older children. Dora showed her biscuit with hundreds and thousands on green icing. Evie confirmed with Rex:

"That's a pikelet, eh?"

Rex pulls the two apart and looks inside

Sina: “Can I see Rex?” (asks twice)

Rex: "Sticky butter" (three times)

Evie opens up her roll and shows the others the jam inside. [20/10/05]

Children also talked about food out of the centre: pizza, McDonalds, what they had for dinner, for breakfast, who had a milkshake machine. James said: "I've got chocolate-I eat chocolate at home-I eat chocolate in my bed". [20/10/05]

Being unwell was, as Brennan (2005) notes, a source of interest. When the teacher brought over Alex's medicine, he said "yummy, yummy" and everyone was allowed to smell it. James said he had a sore head and "spewed" when he was away. Rex displayed the mark from his injection. [20/10/05] 
Possessions were a favourite topic; often it was comparing clothes. James showing his Spiderman shirt led to others displaying what they were wearing. A shark design on a t-shirt led to a description of a shark puzzle at home. James showed his small plastic dragon. Rex's watch led others to describe theirs. Mooloo's comment about buying things led to a conversation about money:

Mooloo has money at her father's that she and her brother earned picking up pine cones .... Rex got \$5 at his party, and he bought the watch at a supermarket. [10/8/05]

Sometimes they talked about centre events. Mooloo told me a teacher made the 'angel wings' she was wearing. After James brought a replica crocodile head to show, he accused Alex of being too scared to touch it, and then admitted he was scared too when he first saw it. Children regularly checked with teachers about whether their name was listed on the whiteboard outside the sleeproom, which indicated they would be having a sleep:

\section{Angus asks about the sleep chart, is his name up? Aidy asks too. [15/8/05]}

They also spoke about family events. Sina talked about a park she visited in Japan, and others mentioned parks. Rex said his father had three babies inside his big tummy, and Aidy corrected him saying only girls could have babies.

Birthdays, and comparing ages, were recurring sources of interest. Maxine said: "It's my poppa's birthday tonight" adding that they were giving him a book. While mealtimes were controlled by teachers, children found opportunities to exercise some control/power. The close seating meant others observed their peers resisting teachers. Evie threw a pair of cardboard binoculars on the ground and ignored a teacher's request to pick them up. They also saw children attempting to exercise control/power over their peers:

James is sitting with Rex and Angus .... When Cassidy sits down, James tells him he can't but an adult intervenes. [7/10/05]

Children enjoyed the responsibility of handing around food, but sometimes used it as a chance to insist on compliance with rules:

When Rex was serving Jeff, he said "fruit" five times and waited until Jeff took fruit. [12/12/05]

Children were also bystanders in more sustained episodes of conflict. Cassidy was at the table when a prolonged episode of taunting by James and Alex reduced Angus to tears. Therefore, alongside the opportunity that eating together offered 
children to deepen their knowledge of each other and to discuss shared interests, it also allowed children to demonstrate their power and/or to be observers of such demonstrations. It was, however, also a place where children showed their concern for others; when Aidy mistakenly sat in Fleur's chair, he relinquished it giving her a kiss and a cuddle.

Busy routines meant teachers often missed out on taking part in these peer interactions, as Alcock (2005) also found, although children's frequent invitations for teachers to sit beside them suggested they were welcome. Prochner (2001) has drawn attention to the messages that may be embedded within the way centre mealtimes are organised; the fact teachers had little chance to sit and talk with children, rarely ate with children, and even more rarely were seen to eat the same food as children, all implicitly contributed to the adult:child demarcation.

\subsubsection{Mat-times}

Mat-time was a regular pre-lunch event, and there was often also one before morning and/or afternoon tea. Sometimes children were divided into two or three groups by age, but more often were gathered in a single group on the mat in the main room. There was also a quiet-time after lunch for those not sleeping. For teachers and children mat-times were explicitly about togetherness, experiences in being part of a community. They were significant as the only occasion when all children gathered in a single group, although the youngest children were typically peripheral participants, from their highchairs for the pre-lunch mat-time, and from the Babysafe for the postlunch quiet-time. Because mat-times were times of high teacher control, they were overt demonstrations of the adult-child demarcation. Children's occasional acts of resistance to teachers at mat-time emphasised that demarcation.

Children appeared to enjoy the experience of sitting together, participating and listening. Being part of the group was in itself an experience of belonging, and also a chance to learn about your peers. For Katie who came once a week, it was a chance to learn who others were, and teachers' frequent use of names assisted this process. Others may have enjoyed it for different reasons. For Robert T., who often played alone, the experience of joining in with singing and music-making was likely to contribute to feelings of inclusion. For a younger child like Anakin, being part of mat-time might be a rite of passage out of the highchair and into being 'big'. 
Children's responses indicated they enjoyed mat-times, in contrast to findings by others (Wiltz \& Klein, 2001). No-one put a sad face on mat-time in the like/dislike activity (Research strategy 3). Rather it was the first positive choice both Ruby and Fleur made, and it was among Mulan's positive choices. However, of the three older boys who did the chart activity, Rex identified it positively the first time but omitted it the second; and neither Angus nor Robert A. included it. (There was no photograph of mat-time in the earlier version which used a collection of photographs rather than a chart.)

Usually mat-time included a story and/or action songs and rhymes. Less frequently it might include musical instruments, a teacher-led discussion or a group game such as “Who's under the blanket”. Teachers' thoughtful preparation was often evident and contributed to making mat-times pleasurable oases of teacher-input within the wider context of self-directed activity. Children watched in suspense as a teacher drew items out one by one from a bag to introduce each activity. Another produced a puppet to demonstrate actions to a song. It was also often clear teachers were drawing on their knowledge of children's interests in their content. A teacher produced a plastic leopard, and talked about differences between leopards and lions; a story about starting school was read. Elements of children's popular culture were occasionally introduced; a teacher displayed a Batman car and demonstrated its features before chorusing "nananananana Batman!" with the children. While each teacher planned their own programme there was a core content that would be familiar to most mainstream New Zealand teachers.

Mat-times were the most controlled regular centre event, and as such were the clearest communal demonstration of the adult-child demarcation. Visually this was embodied in their relative positions, with children sitting on the floor, and the teacher typically sitting on a low chair. Children had little influence on content, although there was a weekly opportunity for them to share items brought from home and they were often asked to suggest songs. Only once did a child have a leading role in a regular mat-time; this was when James displayed his replica crocodile head and, supported by the teacher, showed it to every child. (The child's leading role in the fifth birthday mat-time ritual is described in the next section.) 
Mat-times were prime times when teachers built a sense of community, reflecting Singer's (2003) recognition of how such collective events can be "powerful tools" in building "feelings of solidarity between the children and in the whole group" (p.73). Teachers used them as opportunities to strengthen bonds; they introduced new children and visitors, farewelled those leaving and talked about forthcoming events. While planning documents did not explicitly refer to "communitybuilding" there were other references that suggested teachers appreciated the significance of children developing a sense of interdependence and connectedness.

These gatherings were also forums where teachers created a shared centre culture, a further dimension of community-building. The stories provided shared references; in the playground a teacher commented to children "It reminds me of Mrs Wishy washy", a character in a favourite book. A teacher-made book wove a story around photographs of teachers' cars. Children were delighted to see familiar stories; Angus greeted Hairy MacLary like an old friend. Mat-time was also a chance for revisiting centre events. After the broccoli was harvested, children curled up into 'broccoli' balls and waited for the teacher's touch on their back:

"Look out, here comes Cookie with the kitchen knife, she's going to chop you off to be cooked." [12/10/05]

There was a common repertoire of songs. Highchair children joined in with the actions for songs as they ate; Sina and Amanda knew one song well enough to compose a ribald version together.

Children's sense of belonging within the community was often heightened by having their own moment of recognition. A child might be chosen as a "round bun in the baker's shop" or asked to choose a song. Every child might hear their name whispered and receive a 'high five' as they departed. Hearing children call out "You haven't done me" when the teacher had 'unwrapped' five of the curled-up animal parcels was a reminder of how important such moments of personal recognition are for building esteem (McCadden,1998). The teacher responded and went on to unwrap every child. But it was also a time for understanding you only had one turn, the teacher would not unwrap you twice. 
Mat-times were also significant as the forum where the dialectic inherent in the teaching role was most apparent, as teachers sought to build and maintain a sense of harmonious community without resorting to exercising control in ways that would jeopardise that harmony. Teachers worked hard to make mat-times enjoyable experiences, a task made more difficult by the very different expectations of children in this setting. In contrast to play periods, here the requirement was not to initiate activity, not to communicate with peers, not to access resources, and not to move freely. The mat-time requirements were the clearest example of children learning the expectations of the student role (Apple \& King, 1977; King, 1978; McCadden, 1998). These were lessons in restraint many children found difficult. Coercing and coaxing children to meet these expectations was often a challenge. Although teachers' overt focus was on community, the underpinning dimension of teacher power was never far from the surface.

Teachers used a variety of strategies for initially quietening the group (e.g., "Hammer, hammer hammer" which ends with children whispering) and for dismissing the group (e.g., naming songs). Such transition routines McCadden (1998) believes establish the teacher as the authority. Minor disturbances during mat-times were often ignored or led to positive examples of behaviour being noted. Continued infractions led first to gentle reminders:

"I am just going to wait until Robert turns around and Alex sits up straight” [30/8/05]

and sometimes to more forceful comments. The readiness of other teachers to step in with support suggested teachers understood maintaining control could be challenging and were collectively committed to avoiding the need for overt displays of control. The teacher's embarrassment at shouting at a child and excluding him from mat-time, mentioned in Chapter 6, reflected her discomfort in having momentarily lost her temper.

As noted previously, teachers only occasionally used mat-times as a forum for discussing behaviour. At these times relationships and the ways in which children exercised control/power with each other were explicitly curriculum content.

The teacher... said how sad she was that she had seen hands used inappropriately during the morning. [20/10/05] 
In contrast, other researchers indicate teaching appropriate behaviours is often an element of such gatherings (Skinner et al., 1998).

Mat-time was also a time when a child might witness other children's resistance to teachers. This was most conspicuous in the older boys' reaction to the call for mat-time:

A teacher calls "Haere mai ki te whāriki" ["come to the mat"] and Aidy and Robert A. shriek. Robert dives through the tunnel. Aidy shouts "Climb onto our windows, climb onto our windows" urgently. [4/10/05]

The two boys ran under the fort. Walter, who was coming down the fort stairs, also ran under the fort and he and Robert tucked themselves beside the vertical ladder, while Aidy perched on the window frame.

A teacher approaches and calls "Boys, haere mai ki te whäriki" as she comes towards the fort. She begins to count "1, 2, 3, 4" and they shriek and run behind the fort, but then come around the side. One calls out "I'm going in to morning tea, and the others say "Me too" and "Me too" and head in to the mat.

Children also sometimes resisted the departure process from mat-time:

Angus does not go, but instead looks out the window. Walter also stays in the room. A teacher goes over and together she and Angus look out at the rain. Then after a few moments she says "Please go and wash your hands".

Angus: "I'm a policeman".

She says: "Policeman, please go and wash your hands".

Walter [who is also still on the mat] says "I'm not until Angus". [18/8/05]

Eventually both boys left to wash their hands after teachers had begun serving lunch. Moments of resistance also occurred during mat-time, such as the two episodes involving Anakin and Emjay described in 7.1.1, where it seemed their shared pleasure in their relationship was heightened by their sense of shared rebelliousness.

Finally, mat-time was a place where the use of physical aggression among peers might be observed. Among the older children there were more examples of both full engagement and occasionally also of disruption:

James grabs Alex who is sitting beside him, and wrestles him. Alex cries, and comes across to sit with a teacher where he remains for the rest of the mat-time. Later James hits Jonathan who is sitting in front of him. But when the story is on, he is engrossed. [5/8/05] 
For children who were never observed resisting teachers or using physical aggression, being close observers of such episodes was not an insignificant experience in the ways in which control might be wielded by both peers and teachers.

It is important to keep this account in perspective. All children appeared to enjoy participating in mat-times, and teachers were sensitive to children in planning for and managing them. Nevertheless it was also the site where every child was a close witness to teachers' exercise of control, children's resistance to teachers, and at times to children's use of physical aggression against their peers. These factors contributed to issues of control/power being part of every child's curriculum experience.

While the practice of such teacher-led mat-times is a familiar tradition within New Zealand early childhood education, there are descriptions in the literature of collective meetings with greater child input. References in the literature to children's participation in philosophical discussions (MacNaughton \& Williams, 2004), and descriptions of toddlers taking a leading role in circle time (Emilson, 2007) are thought-provoking examples of giving children a greater role. However, when centre teachers did attempt to do this, it was not always successful:

She is leading a discussion about what are the special things we do at Christmas.... At one stage there is the sound of crying and I hear her say

"You taught him that. You showed him the wrong way..."

Then a few moments later there is an ultimatum "It's your choice, move by Charlie, or join ----'s mat-time. You need to make a choice." [12/12/05]

\subsubsection{Sleeptimes}

Sleeptime contained extremes. Observations showed the after-lunch routine contained some of the most intimate interactions between teacher and child in a day, but very occasionally it was also the site of fierce clashes.

Because the door to the sleeproom was kept closed, children could not include it in their photographs of favourite places (Research strategy 2). However, children's preferences in the like/dislike activity (Research strategy 3 ) showed even older children who no longer slept might remember it fondly. Maxine, Grace, Mooloo and Evie all identified the sleeproom positively; Maxine said: "I like having a sleep". Among the children who still had sleeps, Robert A. and 
Ruby identified it positively, while Mulan and Cassidy did not select it. Angus was the only child who put a sad face on the sleeproom, and his experience will be described to show how issues of power could become a dominating feature of the sleep routine and cause untenable tensions for both child and teachers. It will also show how children may attempt to exercise control in ways that are not playful or oriented to their peers.

When children went to the sleeproom after lunch, the atmosphere was conducive to rest. The muted colours, drawn curtains and dim lighting contributed to the sense of calm. Mattresses were laid out, along with cots for younger children. As children came in teachers guided them to their beds. Children lay themselves down, were tucked in and perhaps talked quietly with teachers and children around them. Teachers ensured children had some control within the sleep routine. Aidy was allowed to keep an engine beside his bed; Mulan was asked if she preferred a cot or a bed.

Most children came readily to the sleeproom, and the following description captures how pleasant the typical routine was for children:

The two teachers pat backs, and piano music plays continuously-it is very peaceful and calm, and the music draws attention away from the still audible voices of children and adults in the rest of the centre.

It is at times possible to hear individual voices, but mostly it is a remote sound.... One by one children go to sleep. Amelia is the last to go to sleep, and that would have taken ... around 40 minutes. Very peaceful process probably no more than 10 soft comments "Lie down ...", "Be quiet ...", all gently and quietly spoken, and all accompanied with back rubbing. [7/10/05]

This routine allowed each child to feel a teacher's soothing physical touch; both going to sleep and awakening were potentially times for physical and emotional closeness between teacher and child. For a child who no longer wore nappies, and who did not initiate contact with teachers, this might be the only gentle close physical contact in the centre day.

Sometimes, despite teachers' efforts, children did not fall asleep:

A teacher came through carrying Mulan out of the sleeproom. Earlier I had heard her come out and consult about the fact that Mulan was not asleep yet. This time she says "she won" as she goes past me. [5/8/05]

As her language indicates sleeping could become a battleground; this was most disturbingly observed in an episode with Angus. His parents were firm in the 
requirement that he regularly slept; a teacher reported his mother frequently rang to check teachers complied with this request. Observations suggested the requirement had become a source of tension for Angus, which mounted during mat-time and lunch routines. The following episode conveys the intensity of the physical and emotional struggle, and the power that both adults and child exerted. Angus:

...goes to his bed, but gets increasingly restless - getting up, waving his legs, talking, rolling around, and sometimes walking around.... The second teacher spends some time with him, patting him, calming him down, then moves to the babies. [12/10/05]

Later, as he struggles, an adult uses the bedclothes to restrain him. He says:

"I don't like you”, "Bad Jelly will get you”, "Bad Jelly will cut you up with knives".

When another teacher took over, she curled around him, and negotiated that if he lay still and silent for 10 minutes, he could get up. He agreed, and she gave him her watch, but he still moved frequently and talked on and off. She says "Do you want me to restart it?" but in the end he was allowed to take his clothes to a teacher who talked about making good decisions while she helped him dress. This was not an isolated event:

As I go to leave I see Angus in his singlet, squeezed into his locker crying his heart out for mummy. [1/9/05]

Nor was Angus the only child who resisted sleeping at times:

Walter had to be carried shrieking through to the sleeproom. [22/9/05]

At a staff meeting teachers shared their deep unease. One teacher described spending an hour getting Walter to sleep, and said:

"I hate it because I feel I am not a nice person”, "a horrible person”.

Another teacher referred to a similar episode with Angus and described her role as "unethical". She said "I felt quite calm, but was holding down the bedding to restrain Angus". [4/10/05]

After long discussion teachers agreed they would allow children to get up if they were not asleep within a certain period.

Such episodes were the most overt teacher-child clashes observed. Brennan's (2005) reference to "lone expressions of independence and resistance [as]... meaningless empty acts" (p.207) seemed inadequate in this situation. Rather, 
Angus's resistance was an intense and private battle he waged with teachers; he appeared to have little regard for how his reactions might be received by peers.

Teachers' expression of their intense discomfort with the situation, as they tried to balance the parents' request with their sensitivity for Angus was the clearest articulation of their uneasy mediation of the dialectic tension that underpinned so much of their work in the centre-maintaining a balance between their sensitive relationships with children, and the need for them to take a controlling role.

\subsection{Rituals}

Finding the point of demarcation between routines and rituals seemed somewhat arbitrary. Birthdays, trips, Christmas and family social events were defined as rituals, in that they were out-of-the-ordinary planned events, which were of particular significance to both children and teachers. Brennan (2005) uses the term "traditional events" and describes teacher-led events such as birthdays, Halloween and Guy Fawkes. The centre ritual described here, the fifth birthday, was significant because it was an occasion that in many ways cut across the arguments that have been presented through these three chapters. Firstly, it was an occasion when the physical and social isolation of the centre was temporarily reduced with the presence of Mooloo's parents participating through the afternoon. It was an occasion when the teacher-child demarcation within the centre was diminished, individually for the birthday girl in the prominent role she was given in the mat-time, and collectively in the shared emotions as teachers and children farewelled her and the family. The significance of this section is that it offers a glimpse of how centre life might be, and provides a platform for the discussion in Chapter 9.

\subsubsection{The fifth birthday ritual}

The significance of birthdays and growing older/bigger recognised in other settings (James \& Prout, 1990; Paley, 1986) was seen here, but fifth birthdays were of particular importance because they also marked a child's departure from the centre. (In New Zealand children typically start school either on, or the day after their fifth birthday.) However, as well as marking the birthday and departure, the ritual can also be read as marking the child's transition from being a young child in the confines of the centre to being a big child in the wider world of 
school. On Mooloo's birthday her clothes symbolised that transition. Her long hair was loose and I noted she was:

... wearing a t-shirt with a sparkly motif, a flounced calf-length skirt, and black shiny boots. She looks very grown up.[19/8/05]

It was an emotional day for her. Several times she seemed upset, and at one stage she cried. At lunch she joined in the conversation about birthdays but ate nothing. She was upset when not chosen to leave quiet-time first; even on this most special of days, she was implicitly reminded she is only one in a community of many. Her parents arrived about 2pm, and having them there, participating in the centre community, highlighted the significance of the occasion, and offered Mooloo a rare bridge between home and centre life. As they sat outside, she alternated between demonstrating skills, like climbing the rope ladder, then returning to sit with them.

Afternoon mat-time began very typically with Mooloo, wearing the birthday hat she had made during the morning, sitting amongst others for the story; but for the rest of the event she sat on a chair beside the teacher, in front of the children, an overt demonstration of her new status. Her portfolio was displayed page by page, before it was handed to her parents. Then,

Mooloo is asked if she wants to choose the song. She does want to but can't think of any, so she is asked to choose a friend to think of one. Evie suggests "Bob the builder", then "Twinkle, twinkle". Mooloo can choose children to leave the mat. She names some children, but others she points at - I wonder if she knows all their names? [19/8/05]

The ritual concluded with the cake that her parents had brought, which was produced after the usual sandwiches and fruit. When Mooloo had blown out the 5shaped candle children spontaneously launched into "Happy birthday". While pieces of it were being handed around, a teacher gave Mooloo the card that had been made. Some children dispersed, but others gathered with teachers to make their final farewells to the family.

The uniting of teachers and children in this ritual, in the sadness of the farewell and in the pleasure of the birthday celebration enhanced the wider teacher-child community; teacher-child divisions were temporarily blurred in the shared emotions the occasion evoked. But hearing teachers later say to children about other events “Oh, it happened on a day when you weren't here" was a reminder that children who did not come every day often missed significant events. A child 
attending daily might feel part of a cohesive evolving community; for a child who came less often the experience could be more fractured.

There were also other layers of meaning embedded within the ritual. The handing over of the portfolio underlined Mooloo's transition from the familiar world of the centre, and her role of 'centre kid', out into the wider world of school. Chapter 5 began with a description of the physical and social isolation of the centre, which restricted contact with the community and with 'real life'. Now that argument comes full circle. That same isolation now meant Mooloo was very unlikely to have any further contact with the teachers or with most of the children in the centre unless her parents initiated it. She might find familiar peers at her new school, but even this was uncertain as the centre drew families from a wide catchment and departing children went to many different schools.

For the children remaining, the ritual was a reminder that one day each of them would leave the centre, that membership of this community was transitory, and that another wider world lay beyond. Some children looked forward to the maturity it implied, and this may have been reflected in children's use of the word "school" to refer to the centre. Jeff says "See how big I am, I'm a school boy now". Rex, who was closer to school-entry age, was not so sure; his mother reported he did not want to turn 5 .

But most significantly, this ritual seemed to contain within it the seeds of suggestions for doing things in other ways in the centre, in ways where the adultchild demarcation was more frequently blurred. Discussion in the final chapter will build on those seeds of suggestion, in considering the implications of the thesis for the profession.

This discussion of the fifth birthday ritual brings to a close this chapter, and the analysis based around the concepts of rules, routines and rituals. This also completes the analysis using the three sensitising concepts drawn from activity theory. Taking each of these perspectives in turn has shown the ways in which the assumed demarcation between adults and children was a fundamental source of curriculum boundaries that limited both teachers and children. But it has also shown that teachers found ways to blur the demarcation, and that children often and robustly challenged it. Taking these three perspectives has also highlighted that relationships with others 
were at the heart of curriculum for children. The dual constructs of establishing relationships with others, and of exercising control over others, were central to children's task of establishing their identities within the centre, and so were concerns which children introduced into the centre curriculum. Although there were many other ways in which children influenced the scope of curriculum, the focus on the exercising of control/power was the most unexpected.

To return to the image of concentric circles which was used in the first chapter (p.2), these three chapters have explored the area between the first and second circles, the area that constitutes the unintended, unplanned and often unacknowledged aspects of curriculum, and have considered how children experienced and influenced the boundaries. The next chapter describes what lay beyond the outer border, what constituted the null curriculum (Eisner, 1985), and how and who defined this. 


\title{
CHAPTER 8
}

\section{THE NULL CURRICULUM}

\author{
As I was walking up the stair \\ I met a man who wasn't there \\ He wasn't there again today \\ I wish, I wish he'd stay away. \\ (Mearns, 1875 - 1965)
}

In this chapter attention shifts to what was excluded from curriculum and to how that process of exclusion occurred. The subsidiary research question guiding analysis here is:

What potential aspects of learning and teaching, i.e., the null curriculum, are being excluded from the curriculum that young children experience and enact?

Discerning the boundary between 'appropriate' and 'inappropriate' was challenging because it required looking for absences, listening for silences. Consequently it must be acknowledged that at times the data here were less substantial than in previous areas of analysis; collating the data sometimes felt like catching feathers. It was made more challenging because it required stepping outside my own assumptions, as well as those of the profession. Yet abandoning assumptions meant curriculum possibilities were "virtually infinite" (McCutcheon, 1997, p.189). In order to contain the topic the "curriculum universe" (Flinders et al., 1986, p.38) was defined as: what was present or on the fringes of being present, but was disregarded, avoided, closed down or denied by children and/or teachers. This reflected the definition of null curriculum adopted for this thesis:

Curriculum that arose, or potentially arose, in the setting, but that children and/or teachers deemed to be inappropriate. (Chapter 2, section 2.1.6)

In thinking about what might be null curriculum in the centre, making comparisons proved a useful point of leverage. Descriptions of early childhood education in the literature made international comparisons possible, and these often offered alternatives to familiar practices. A diversity of services within New Zealand, with different philosophical approaches but with a shared commitment to implementing Te whāriki, made cross-service comparisons possible, for example making comparisons with Montessori or Steiner settings. There were, and still are, 
perplexing questions. Where is the distinction between inappropriate and irrelevant? How do you distinguish between what children have learnt not to do, and what they define as inappropriate, or is there no difference? What else in my own null curriculum was preventing me from seeing more?

A central argument presented here is that there were differing definitions of what constituted null curriculum. Previous descriptions have assumed the teacher/policy maker (Carpenter, 2001), and perhaps also the parent (McCutcheon, 1997), as the agent/s of exclusion, but the commitment to children's voices in this thesis meant they were the logical starting place. It quickly became apparent that what was null curriculum for teachers was not necessarily so for children. It was also found that individuals varied in their interpretation of null curriculum, reflecting the social mores of the other worlds in which they moved, and the identity they were currently establishing/inhabiting within the centre. That teachers' and children's definitions differed is a reflection of the first theme of the thesis, the adult:child demarcation, but more particularly, it is a demonstration of the second theme, because the differences between definitions of what constituted null curriculum arose largely from children resisting adult norms as a way of strengthening their own peer relationships.

What was null curriculum for children, and what they perceived to be null curriculum for teachers, are considered first. What constituted null curriculum for teachers is then described using the framework of content, intellectual processes, and emotions. Aspects of curriculum that seemed to lie on the border between appropriate/inappropriate for teachers were labelled 'borderline' (see Figure 10). 


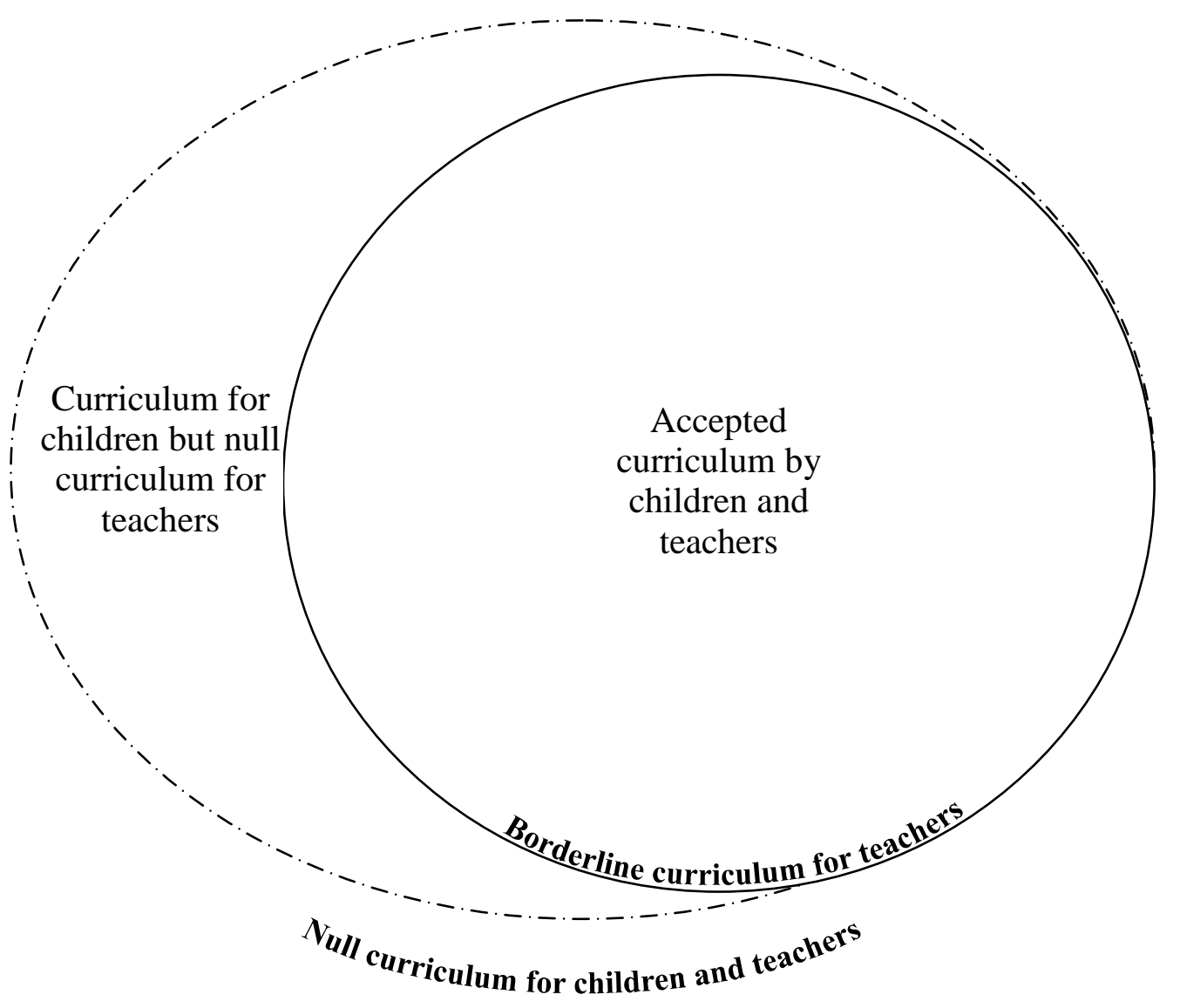

Figure 10: The differing boundaries of null curriculum for children and teachers

\subsection{What was null curriculum for children?}

No references to children's/students' definitions of null curriculum were found in the literature, but this was my starting place. Did children themselves set boundaries to the curriculum? They appeared so catholic in their interests and ideas it seemed unlikely anything that potentially arose would be discarded as inappropriate. Although these were not strong trends in the data, two aspects that seemed to be null curriculum for children were their bodies and naming another child as disliked.

\subsubsection{What was null curriculum for all/most children?}

Children's own naked bodies were largely null curriculum, a finding anticipated by Sanson $(2007,2008)$ in writing about dance, and in literature addressing issues around children as sexual beings (e.g., Silin, 1995; Surtees, 2003; Tait, 2001). Children frequently changed in the locker room, but nakedness was a momentary space through which they were moved/moved themselves. There seemed to be tacit agreement that unclothed was not ok. When Evie, so often the rebel, stripped to the waist she was told by a teacher: 
"I don't want your body to get burnt by the sun. I need you to go and get a t-shirt on." [26/10/05]

Although children changed together, were in the bathroom together, watched others having nappies changed, they almost never commented on each other's bodies, which was in sharp contrast to the shared interest in clothing among older children. A rare exception was when James looked down David's pants and said "David has a bum". Yet in the 1980s running naked through the hose was an unexceptional event in my children's centre experiences, and a photograph from the 1930s shows naked "sunny bunnies" in an Auckland kindergarten (H. May, personal communication, October, 10, 2008). While a detailed exploration of these changing perceptions lies beyond the scope of this thesis, the depth of shift seems to indicate more than protecting children from exposure to the sun. Wider concerns arising from issues of child abuse (Duncan, 1998, 1999) and the increasing discourse of risk within society (Tait, 2001) are also likely to underlie these changed notions of acceptability.

Yet, observations in the centre suggested a latent curiosity about others' bodies existed. Cassidy was curious Jeff's doll did not have "a button" on its stomach. Two sisters, on separate occasions, conducted almost clinical examinations of my face. The fact that Charlie, who was taking photographs of favourite places (Research strategy 2), said "No" when Evie took off the top layer of the pregnant woman puzzle so the baby in her belly was visible, was a shred of evidence that for Charlie if not for Evie, the clothed figure was more acceptable. Evie later took her own photos with the naked figure and baby showing. It seemed only a few children showed curiosity about others' bodies; most were not curious or had learned not to express such curiosity. Given the reported evidence of children's curiosity about bodies (Isaacs, 1933; James et al., 1998; Prout, 2005) the latter seems more plausible.

On the five occasions when I asked children if there was anyone they did not like to play with, their responses suggested this might be an uncomfortable topic. Grace and Maxine whispered together, before Grace whispered three names in my ear. Cassidy's first response was a very quick and repeated "No", although, as described in Chapter 6, he later talked of hating "monsters". Sina and Rex both answered with a "No". Yet parents readily named children whom their child complained about, and it was not uncommon for children themselves to exclude others. Was children's reluctance to label peers unfavourably to an adult a tribute to the energy with which teachers worked to create a positive feeling of 
community? My sense that children felt such conversations in the centre were inappropriate suggested they had absorbed this construction. However, the fact that I only asked children this question on five occasions reflects my own discomfort, my own assumption that the community should be harmonious. My unease may have been conveyed to children. On the rare occasions when children did express negative feelings to me about others, they chose private moments; Jeff and I were alone when he told me "Robert A. does lots of mean things to me". Earlier chapters have included examples of children explicitly and implicitly rejecting others, but only once was a child heard to say "I don't like you" to a peer:

When Aidy called "Come on, come on guys, do you want to look at my brand new truck?" Evie said "No we don't because we don't like you eh, we don't like you" and followed it with a cackle of laughter.

Aidy's response was to move away with his truck saying:

"I'm going to put my truck away 'cause it's broken, eh? It's broken. Stupid old truck."

He abandoned it, climbed onto the fort where Angus was, and called down to Evie:

"You're naughty, we're going away from you." [18/10/05]

His departure and his rejection of the truck indicated the impact of her rejection; his use of "naughty" suggested he recognised her statement as inappropriate in this context.

Two factors may have contributed to children's reluctance to express dislike for others. Other researchers have noted the characteristically positive, nonconfrontational approach teachers habitually take both with each other (Nuttall, 2004; Norberg, 2006) and with children (Brennan, 2005; Woodrow \& Fasoli, 1998), and the maintenance of a harmonious group has been identified as a deep-seated value within early childhood (Brennan, 2005; Rivalland, 2007). The same positive teacher behaviours, and the prioritising of community harmony were evident in this centre, meaning that the roles teachers took would have reinforced the inappropriateness of expressing dislike for others. Children's apparent reluctance to label others as disliked playmates may also be an indication of their dominating motivation to establish and maintain social relationships (Corsaro, 2003); it was argued in Chapter 6 that relationships were the core of curriculum for children in this setting. 


\subsubsection{What was null curriculum for some children?}

Was null curriculum shared across the whole group of children, or were there differences reflecting age, gender, or individuals? The only age-related aspect of null curriculum was the unexpected finding, outlined in Chapter 6, that the concepts of learning/teaching seemed to be null curriculum for older children. There seemed to be no other age-related division, beyond the limits imposed by physical mobility and verbal skill. Younger children, within the limits of their mobility, had free range of the environment for most of their waking day, and were peripheral participants in mat-times. Theoretically, older children had restricted access to the gated Babysafe area, but they often visited this space, and those toys often migrated past the fence. Older children were occasionally offered a cot to sleep in. Two-year-olds Jordan and Thomas were sometimes part of the superhero play.

Were there differences in what constituted null curriculum that reflected gender demarcations? Observations had shown that superhero persona, and the boys' superhero play, was not entirely null curriculum for girls. One distinction was that when girls participated they never physically attacked others. The fact that there was only one occasion when an older (aged 3 or 4) girl was observed hitting another person suggested physical aggression might be an aspect of the boys' interactions that girls defined as inappropriate. Dora and Charlie's reported enthusiasm "to do play fighting" when the gym mats were available, suggested light-hearted rough-and-tumble play was not unacceptable at least to them.

In contrast, some of the older boys (aged 3 and 4) never engaged in some activities favoured by girls. Alex, Walter, James and Rex were never seen playing with dolls. Neither was Evie but then, as Chapter 6 showed, she energetically resisted the stereotyped girl role. There was no Barbie doll in the centre, but she was occasionally present in girls' conversations. The only time boys talked about Barbie, was in a ritualised response game Rex, Angus and Aidy played with Dora, which provided further evidence of their positioning of dolls, including Barbie, as not-boy and not-acceptable:

They have set up a routine where she says "You've got a Barbie" and they cover their faces with their hands immediately. She says it about 15 times-with pauses - and they react with glee each time. When she pauses Angus says "Come on, come on, come on girl" and she starts it up again. [16/12/05] 
The pattern of boys dissociating themselves from activities perceived to be girls' is widely reported (Browne, 2004; Corsaro, 2003; Keddie, 2000; Paley, 1984) indicating that gender may be a frequent ingredient in defining what constitutes null curriculum for an individual.

Glamorous dress-ups were another aspect of the girls' world that was null curriculum for some boys, although flowing capes were often worn as superhero insignia. For many girls, a focus in dressing-up or face-painting was to look beautiful, but the aim for boys was typically to appear fierce and threatening, as Jarvis (2007) also reports. This was also demonstrated in their menacing handclawed gestures, their use of tools and arms as guns, their blocking body stances and their excluding verbal messages. (However, they did participate in having their hair gelled by a teacher one afternoon.) These two kinds of resources, dolls and glamorous dress-ups, seemed to be null curriculum for many boys.

There are inevitably questions that remain unanswered. Reflecting on the determination with which many older boys explored their ability to threaten highlighted the challenge boys faced in defining themselves as male in a womandominated world. Was being a male null curriculum? Girls had nine female adult role models in the centre, boys had none. How would the centre have changed if there had been a male teacher? Or if all the adults had been males? While the patterns of boys' behaviour seen here are widely reported (e.g., Corsaro, 1997; Fabes et al., 2004; Longwell-Grice \& Letts, 2001; Paley, 1984; Watson, 2005), it seems likely that the void in male role models may partly explain why so often boys seemed to explore their maleness in a stereotyped way focused on being 'other than' females. Did teachers' commitment to emotional warmth and harmony and their avoidance of overt control strategies accentuate aggression as the 'other', and therefore non-female, behaviour in this setting?

Were there individual differences in children's definition of what might be null curriculum? Comparing children of similar age and sex suggested there were. James seemed often to be motivated by a desire to control, and for him, being weak or a follower among his peers was currently null curriculum. Rex, the oldest boy in the centre, also wore Spiderman regalia and joined in the running/shooting games but he regularly interacted with girls and was never observed hitting or being hit. His actions showed he chose to define physical fighting, which was part 
of the curriculum currently on offer, as inappropriate and therefore as null curriculum. There are a myriad of other examples which yet again reinforce the individual nature of each child's experience. Earlier analysis had emphasised how vastly different a day might be for two children; here analysis underlined the differences in what children might currently define as being inappropriate/irrelevant for themselves.

\subsection{What children considered to be null curriculum for teachers}

The second aspect of null curriculum lies on the boundary between appropriate and inappropriate. It is the topics and language children assessed as beyond the bounds of appropriateness for teachers, yet chose to use, often as an expression of resistance. Teachers' reactions varied. A strong negative reaction confirmed that it was inappropriate, but more often teachers accepted or at least tolerated the language. Given the strength of exclusion around the naked body, it is perhaps not surprising that all these topics related to the body. The use of scatological language was the most obvious example.

The ways children sometimes used "bum", "wees" and "poohs" suggested they believed teachers defined them as inappropriate. When James was rebuked he said "gross as", and elaborated "gross as wee, pooh, bum" before riding away [18/8/05]. Only occasionally did a teacher's reaction indicate these words/topics might be null curriculum:

"If you say those words again you'll have to go inside." [9/8/05]

Chanting such 'forbidden' language together was a way of engendering a feeling of togetherness. Aidy, Robert A. and Walter chanted "Finger bum stink" as they kicked the back wall. Sina's and Amanda's refrain:

"bum bum bum bum" then "pooh pooh, pooh pooh" [16/12/05]

was a rare example of girls using language in a similar way. Older boys used this language in taunting others: Alex and James murmured "you're poohey" to Angus. However, children also used this language unselfconsciously as part of their regular conversation: "I need to go pooh", "going wees". Teachers used it similarly; for example, an entry in Cassidy's notebook read:

We are encouraging Cassidy to tell us when he has done a pooh in his nappy. [Nobk47201] 
But teachers did not use this language in reference to themselves, in fact it was not a topic they discussed. An exception occurred when a teacher read a story about Mr Mole trying to identify who had produced a strange-shaped pooh (Holzwarth \& Erlbruch, 1993). It seemed scatological language might constitute borderline curriculum for teachers-aspects of curriculum they acknowledged but rarely extended because they ran counter to wider social mores. It therefore seemed there was misalignment between children's and teachers' perceptions. Although teachers occasionally classified such language as inappropriate this was highly dependent on circumstances. But it seemed children found it satisfying to define this language as forbidden and make use of it in acts of defiance to adults, and in strengthening peer bonds. Others report similar use of such language (Alcock, 2007; Kyratzis, 2004). Using it to taunt peers, seen here occasionally, is also noted by Corsaro (1985).

References to other bodily functions seemed similarly blurred. Mooloo came to me to show me a glob of something on her hands she described as "sneeze", which I understood as an effort to shock, particularly when she ignored suggestions to wash it off. Boys demonstrated burping, and made comments like:

"My mummy burps", "My mummy farts” [9/8/05]

in ritualised exchanges. Teachers typically ignored or distracted children when they were aware of such exchanges, and did not discuss their own bodily functions. Again however, a teacher read a story about a dog whose overpowering farting saved the day (Kotzwinkle \& Murray, 2001), thus acknowledging children's fascination with such topics. It seemed reading books provided teachers with a 'safe' way to engage with borderline topics that were at odds with social mores and perhaps with their personal values.

Boys' references to female bodies, pregnancy and birth in their exchanges suggested they sometimes positioned these as 'forbidden' topics.

"My mummy's titties”, “My mummy has two babies in her”... “My mummy has three babies". [9/8/05]

Babies and births were a hot topic for teachers, parents and children because a family was looking forward to a multiple birth. Teachers readily followed up children's references with conversations that focused on such details as whose mother was pregnant, when the baby was due, and whether it might be a boy or girl. The details of how pregnancies begin-intercourse and the formation of the 
ovum-were never mentioned. These were, as will be seen below, details that were null curriculum for teachers.

\subsection{What was null curriculum for teachers}

What constitutes null curriculum for teachers is where other discussions of null curriculum begin (Carpenter, 2001; McCutcheon, 1997). There was a variety of ways in which the boundary of teachers' definition of appropriate curriculum could be deduced. There was content teachers concealed from children, possibilities they closed down, topics children raised but teachers deflected, aspects of play they tolerated but rarely engaged with, and teaching/learning routes they did not take.

\subsubsection{What was not null curriculum for teachers}

The first finding was that teachers blew away many preconceptions about what might be null curriculum for them. They entered into the children's world and largely delighted in their ideas and interests. For example, teachers represented children's popular culture in the centre, so ensuring it was not an area of null curriculum as others had so frequently reported (Fleet, 2002; Giugni, 2006; Jones \& Nimmo, 1994; King, 1978; Ryan \& Grieshaber, 2004). While watching TV or videos was not a centre activity, elements of what children liked to watch at home were reflected. Images of Batman, Barbie, Nemo, Winnie the Pooh, the Australian music group Hi 5, and the New Zealand rugby hero, Tana Umaga were on the walls. There were Spiderman and Batman dress-ups, and Thomas the Tank Engine trainsets. Children and teachers sang "Bob the Builder", and listened to "Bad Jelly the Witch" and "The Lion King". Many of the videos and TV programmes children mentioned as favourites were covered in this range.

Long-standing traditions have shaped ideas about teaching practices and content. Two areas of particular contention have been the introduction of school-type learning and the role of teachers in children's creative art; in these areas my preconceptions about what might be null curriculum were dispelled. While there is continuing debate over introduction of academic subjects into early childhood education, including the formal teaching of reading and writing (e.g., Katz, 2007), in this centre instruction in letter formation and reading was not null curriculum. While it was not a frequent event, there was a displayed chart recording an 
episode where children had practised the letter ' $\mathrm{t}$ ', and planning documentation included references to children taking early steps in learning to read:

During Yellow group mat time we have been using our "beginner reader" books. The sentences in the story are repeated each page with a slight variation, and are simple and short. [Nobk921d1]

A recommended role for teachers of encouraging but not intervening in young children's art has until recently been accepted practice for New Zealand teachers (Brownlee, 2004). With increasing emphasis on sociocultural teacher practices, and growing awareness of the model of the artelier in Reggio Emilia (Gandini, 2005), a more proactive role for teachers had been advocated (Farrant \& Wright, 2007). At times these teachers took that more involved role, working alongside or collaborating with children. Teachers and children worked together creating an underwater mural; a group of girls gathered around a teacher who was drawing, commenting and suggesting details she might add. Such episodes were striking for the sense of camaraderie between teacher/s and children, which enhanced the feeling of teacher-child community.

The remainder of this discussion on what constituted null curriculum for teachers is organised into the three areas that provided the framework for the literature review in Chapter 3: content, intellectual processes (Eisner, 1985), and affect (Flinders et al., 1986). Further aspects that were not null curriculum for teachers are touched on through this discussion.

\subsubsection{Content that was null curriculum for teachers}

Choosing to conceal potential curriculum content from children provided the clearest examples of what constituted null curriculum for teachers. There were two examples of this.

The teacher came to the door of the deck with something in a piece of paper. She said "Guess what they found in the mulch?" An adult said "A pooh?" and she said "No", and spelt out "A bird's head". She said that she assumed something had eaten the rest. [28/9/05]

Her decision to let children believe it was cat excrement, rather than a mutilated dead bird, was an example of the desire to protect children from the unpleasant (Silin, 1995). Although she told me dead birds had been shared with children in the past, observations showed death was a topic teachers rarely broached. This was in stark contrast to the frequent references to shooting, dying and killing in the older boys' play (Corsaro, 1985, 2003; Paley, 1984; Watson, 2005). Even in 
the familiar mat-time rhyme of "Five little monkeys" which the crocodile "snapped", it was a child who made their fate explicit, saying "This is where the dead people go" as she moved the monkeys to the corner of the magnetic board. Such teacher uneasiness around the topic of death is widely reported (King, 1978; Silin, 1995; Woodrow, 1999), and linked to images of children as innocent and needing protection (Woodrow, 1999).

That the two-child car, which was a permanent stationary feature of the playground, could move was concealed from children. The unexpected discovery of its potential came when visiting schoolboys pushed children around in it during the carol-singing evening. This was a conspicuous example of a consistent teacher practice described in Chapter 5, the defining of how equipment could be used. Defining the car as stationary meant children could not explore the relationship between the movement of the steering wheel, tyres, and trajectory, experience the physical exertion needed to shift it or the responsibility of steering to avoid others. While defining it as stationary was professionally justifiable in terms of management, supervision and safety, it nevertheless closed off a potential aspect of curriculum for children.

As noted above, some curriculum topics introduced by children were tolerated but not supported by teachers. While teachers saw participating in children's activities as part of their role, their role in relation to superhero play, as noted in Chapter 6, suggested ambivalence. When a teacher assessed that play was too aggressive she refused to tie a cloak around a boy's neck but said he could wear it tied round his head "pirate style", and the gym mats were put away to stop children wrestling. There was only one observation of a teacher taking a leading role facilitating noisy wild animal (lions) play with the older boys. Did teachers' lack of participation in superhero play indicate it was less valued, and bordered on being null curriculum for them? Did they sense, as Watson (2005) and Browne (2004) suggest, that in this play boys were defining themselves in opposition to female teachers? There are ideas within the professional literature of strategies for engaging with superhero play (Carlsson-Paige \& Levin, 1990; Watson, 2005). Could teachers have challenged those elements of threatening and physical aggression that sometimes occurred within the superhero play if they had engaged with it more proactively? Did these teachers believe their involvement would not be welcomed? Certainly they were not invited to participate. 
The use of 'guns' is banned in many early childhood education settings because of their connotations with violence and killing, and because of their role in boys' demonstrations of power (Davies, 1989). Here, however, the older boys' use of hands, arms and a range of resources to represent guns was usually accepted as an integral part of their play. Again, teachers' only engagement with them was in a monitoring role, suggesting this also was borderline curriculum. On the occasion when a teacher helped children make rolled paper swords, she took only a supervisory role in their use.

Physical aggression was sometimes a feature of older boys' play. Although it is regularly reported as a characteristic of boys' play (Fabes et al., 2004), physical fighting is so at odds with assumptions about what is valued in early childhood education (Gonzalez-Mena, 1997) that it may seem absurd to consider it potential curriculum. Observations showed rough-and-tumble play was an area bordering on being null curriculum for teachers, as it would be for most teachers in New Zealand centres (Watson, 2005); it was usually stopped, occasionally ignored, but never extended. It is, however, not inconceivable to imagine children's interest in physical combat being supported in ways that might extend and complicate their interest in physical prowess: demonstrating tae kwon do kicks, practising how to fall and roll, introducing a punch bag. Watson (2005) describes children and teachers making a superhero video in which children role-played "stylised fighting scenes" (p.37). Again, one wonders how different it would be in the hypothetical centre with only male teachers. The finding that rough-and-tumble play may have benefits for brain development (Hughes, 2003) is cause for thought. Even more interesting, in the context of this thesis, is Jarvis's (2007) comment that rough-and-tumble play offers children the chance to practise "spontaneous and autonomous competitive and co-operative interactions simultaneously, developing complex social skills that fundamentally underpin primate adult life" (Jarvis, 2007, p.185). Unexpectedly, that wording provides affirmation for an aspect of the second emergent theme of the thesis: the argument that a tension between being accepted and exercising control characterised many relationships in the centre. 
Children's use of sexual allusions suggested they understood this was null curriculum for teachers, and indeed teachers did not respond. The fact Mooloo waited until I left the room before she said into the tape recorder:

"Please, welcome to our house, please, you have to come to kiss me (pause) and then ----- [indistinguishable] you please won't you, please marry me" [17/8/05]

suggested she saw it as risqué. Most frequently sexual allusions were used in peergroup chanting sequences. Sina and Amanda's improvised song included: "boyfriend, boyfriend," and "my mummy got a boyfriend called Papa" [16/12/05]. James, Rex, Alex and Walter, on the top of the fort, participated in an exchange interspersed with wild laughter:

“My mum put on lipstick and kissed my dad".

"My mum kissed my dad".

"My mum and my dad kissed and she had lipstick".

"My mum put lipstick on my dad and then they kissed". [14/12/05]

The site they chose and their laughter indicated they saw these topics as less/not acceptable to teachers. The fact Rex called "Hey Alison, do you know what?" and repeated the last statement showed he at least did not see it as inappropriate to share with a non-teaching adult. As in all aspects of children's defining of null curriculum, definitions of inappropriate/appropriate from home contexts would have influenced their assessment of the demarcation in the centre.

The topic of sexuality was not broached by teachers. Given the deeply embedded social mores, it was not surprising to find teachers did not refer to children's, or their own, sexuality; this reaction is widely reported (Blaise \& Andrew, 2005; MacNaughton, 2004; Robinson, 2005; Surtees, 2003). Teachers' silence in response to actions and allusions that were potentially sexual from children suggested teachers positioned children as asexual. However, as noted above, there were no observations of children exploring their own bodies nor, perhaps surprisingly, of children masturbating. But children did use their bodies to express their emotions physically. When Evie and Cassidy were together in the little tent:

Evie lies full length on top of Cassidy for a few moments, then they lie next to each other in the cushions, giggling and laughing. [22/9/05]

Children hugged each other. Angus and Dora chose to have their photo taken giving each other a full bear hug. Children hugged adults; Aidy gave me a full frontal hug when I was sitting on the mat and kissed me on the lips. I recognised 
unease in my own reaction to children's sustained embraces, but children's willingness to share them suggested teachers might be comfortable accepting them. One further aspect of sexuality relates to the images of sexuality portrayed. Recent articles have challenged teachers to portray a wider range of family types and sexual orientations (Gunn, 2006; Robinson, 2005; Ryan \& Grieshaber, 2004; Surtees, 2003). In this setting there was nothing that challenged the pervasiveness of heterosexuality, although teachers' sensitivity to, and acknowledgement of, children in reconstituted families suggested they would have supported other examples of family diversity if this had been part of the wider centre community.

Although teachers' efforts and energy allowed the fringes of real life to creep in, as was indicated in Chapter 5, many aspects of the real world were null curriculum. There were five aspects of data that bordered on this topic: visitors, outings, children's interests, teachers' interests and children's involvement in real tasks.

There were many visitors-parents, siblings, grandparents, teachers' families, past teachers, tradespeople, health professionals-but only a few actively interacted with children: the policeman who brought the dogs, a teacher's aide who came to work with a child, the health nurse, and teachers' children, who were invariably a focus for attention if they were present. But there were many other people who might have come: school teachers, school classes, next-door neighbours, musicians, artists, potters, story-tellers, actors, family members sharing traditional food, stories or songs. Such visitors would have diminished the isolation of the centre from the wider community.

Trips out of the centre were highly controlled but intensely exciting events. Older children visited the school, several groups visited a walled garden within the worksite grounds, a group visited the construction site in the parking area. Even a 30-minute trip in which children travelled no more than 400 metres offered opportunities for picking daisies, running races, climbing trees and jumping from them, playing hide-and-seek, and collecting dropped camellia flowers and gum nuts to use in collage. Seeing the excitement generated among children at the prospect of even such a short local trip underlined how confined the centre might feel. 
A further aspect of the real world related to children's interests. Identifying and working with these was a priority for teachers, but as described in Chapter 6, only those interests that overlapped with the range of experiences regularly offered were easily supported. Aligned to the invisibility of some children's interests, was the lack of visibility of teachers' interests. Although teachers included photographs of themselves and their families in the whānau (family) book (a resource teachers were making that was to include photographs of teachers' and children's families), in other ways it was surprising how little they shared of their own interests and skills, a finding reported from other New Zealand settings by Manning (2008). Evidence within the centre showed interest or skills in: art, baking, carpentry, Christmas light displays, gardening, guitar playing, horseriding, and photography. Teachers told stories about their families, family members called in occasionally, and one teacher shared photographs of a family trip to Disneyland. However, when I reflected on how little children knew about them, and their out-of-centre lives, it seemed teachers might not see their own interests as appropriate curriculum content.

The scarcity of real tasks for children, and their limited access to 'adult' resources, were described in Chapter 5, yet the value of participating in such activities is recognised (Jones, 2005) and examples exist in other New Zealand settings. For example, from visiting students in centres, I know children in Steiner kindergartens regularly bake bread and that they wash dishes in Montessori centres. The only real task that was regularly part of the curriculum for centre children was tidying, and yet this task was one children resisted. Was this because it ran counter to their enthusiasm for continued play? Or was it because it provided a focus for playful expression of resistance to teachers?

In each of these five aspects of the data that broach the issue of the real world as null curriculum, teachers were proactive in extending the scope of curriculum for children. They did invite visitors, organise trips, support children's interests and share their own, and introduce real tasks. However, the setting apart and separation of young children from the adult world was so structurally embedded, that their best efforts could do little to bridge that division. 


\subsubsection{Intellectual processes that were null curriculum for teachers}

Were there intellectual processes, as Eisner (1985) suggests, that were marginalised by teachers in this setting? The line of reasoning used in constructing an answer to this question winds through the following topics: teachers' engagement with children's social skills, the kinds of learning prioritised in written documentation, pressures contributing to less emphasis on some aspects of children's thinking, opportunities for children's participation in decisionmaking, and teachers' role in challenging gender stereotypes.

A recurrent theme throughout this thesis has been recognition of the energy and commitment with which teachers worked to create a positive community. In their sensitive and empathetic relationships with children they modelled social skills, and collectively and consistently they reinforced the need for children to learn to consider and co-operate with others, to modulate their own desires, and to adapt to centre routines. This emphasis on social skills, which also featured in teachers' planning documentation, indicated their commitment to "fostering children's social skills and collective endeavour" (Ritchie, 2001, p.137), and suggested they recognised the importance of children's peer relationships.

It seems important to try to discern, beyond the focus on social learning, what other intellectual processes were advocated and valued? Twelve children shared their portfolios (Research strategy 5) and these have been used, along with observations, as the primary data sources here, because they provided a collection of learning stories and images intended to capture the emerging skills, strengths and interests of each child. Comparing the portfolios with teachers' six-weekly plans, and with children's notebooks, showed the portfolios covered a wider range of teaching/learning, with less emphasis on social skills. What did they suggest was valued?

The first impression was that artistic expression was highly valued. Each portfolio contained a significant number of children's artworks, proportionately more than might be expected from watching children's day-to-day activity. These were mainly paintings, but often also a variety of other media (e.g., collage, drawing). Children often turned these pages without pausing, perhaps because many paintings were folded and so not clearly visible; only two children indicated an artwork had particular significance for them. Photographs of creative works such 
as block constructions and cardboard box creations were more likely to receive comment. Other forms of creative expression captured less frequently were dance and music. Teachers' use of stickers and hand-drawn motifs, such as stars and smiley faces, to decorate the pages provided a thought-provoking counterpoint to children's artistic expression. Sina paid more attention to these decorative elements, particularly the stickers, than to her own artwork.

A varied range of physical skills, such as cutting with scissors, threading beads, swinging, climbing and ball skills were prioritised in photographs and accompanying notes. Written text explained children were developing such skills as accuracy, precision and competence in manipulative skills, hand-eye and footeye co-ordination, agility and balance. There were references to children developing dispositions such as determination, persistence, accuracy, competence and concentration, reflecting teachers' professional commitment to using the dispositional framework underpinning the learning story assessment framework that links with the Te whäriki goals (Carr, 1998, 2001). Many of these references were in the context of physical tasks.

There were frequent references to exploration as an aspect of children's learning, reflecting its position as one of the five strands of Te whāriki. There were descriptions of children exploring materials and the changing state of substances, such as sand and water, and to exploring maths and physics concepts through activities such as cooking, bike-riding and sandplay. This focus on exploration reflected teachers' philosophical commitment to children's freedom to make choices and access materials and equipment as needed. The exploration possibilities seemed vast for young Macauley who was just learning to walk. For Rex, who had been at the centre for years, it seemed that the social world might offer the greatest remaining opportunities for self-guided exploration.

Fewer portfolio entries referred to children's own ideas. In comparison to the many references to "exploring", there were only occasional descriptions of "discovering", or of children developing their own theories. Examples included descriptions of: a fascination with a particular story leading a child to recreate it with blocks and art materials; a child 'reading' books independently; children exploring transporting and trajectory schema. When the scope of data was extended to include all planning documentation for the year, there were further 
examples of children's intellectual skills. For example, Aidy's knowledge of the Thomas the Tank engines and of the words and music of "The Lion King" were noted, a pattern of trajectory schema was identified for Robert T, and a conversation about families was recorded to illustrate Cassidy's depth of understanding and mathematical skill. However, records focusing on children's thinking were in the minority in the portfolios and other documentation.

Observations showed many examples of teachers engaging with children's thinking. Frequently used strategies were: pointing things out to children, "When you put your hand in, water comes out"; sharing their own thinking, "It is stuck" [one container is stuck inside another]. "Banging it on the table, what will happen?" [as the teacher bangs it on the table in an attempt to dislodge it]; and, most particularly, asking children questions, "So how long will it take you to get all the water from that trough into this trough?" "What happens if you put one more in there?" The prioritising of questioning was unsurprising in light of references in the literature to the predominance of this as a teaching strategy (Bernstone, 2007). In quiet moments of interaction between one or two children and a teacher, such questions often prompted children to recall events and share ideas, and there were many examples of sustained satisfying exchanges. When it was busier the teacher's attention often shifted before a child answered or reacted. Indeed, at times there did not seem to be an expectation from either teacher or children that there would be an answer. Asking the question seemed sufficient. It was rare for a child's ideas to be challenged, unless those ideas impinged negatively on their behaviour within the community.

Observations had also shown examples of teachers sharing knowledge and/or demonstrating a variety of skills to children. For example, teachers did such things as: demonstrate sign language, use te reo Māori and Samoan language, read a book about magnets, show how to draw smiley faces, discuss a poster about transport, describe the physical actions for swinging, discuss what was happening on the building site, create a book about horses, demonstrate the use of a funnel and waterwheel, point out the features of the crocodile jaw. They taught new songs, rhymes and games at mat-time and read a multitude of stories. However, as these examples indicate, teachers rarely shared a depth of content knowledge with children. The teacher-made book about horses was an exception. The overall impression was that teachers did not pursue children's thinking with the same 
assiduousness and purpose that characterised their approach to social learning. Yet my professional judgement was that these teachers were committed professionals who were providing a programme that would compare very favourably with other early childhood centres, and the positive Education Review Office review they received endorsed this.

What factors might contribute to a relative lack of focus on children's thinking? At a concrete level, the use of photographs in recording children's learning (and they were the feature of portfolios children found most attractive) tended to result in a focus on activity. It is harder to photograph a child being curious, reasoning, or planning. Environmental aspects also worked against children focusing on challenging intellectual activities. The multiple distractions in the busy environment, the periodic requirement to tidy up, and the lack of space to store ongoing constructions were not conducive to concentration or to long-term projects. The many demands on teachers meant organising and supervising routines, and monitoring behaviour sometimes took precedence in their interactions with children.

There are also more philosophical issues that may have influenced teachers' responses to children's thinking. The first relates to teachers' attitudes to schooltype learning. As mentioned above, there is a widely held resistance to the infiltration of school-type teaching methods into early childhood education. The following passage is often quoted, almost as a mantra justifying the position:
Just because children can do something when they are young does not mean they should do it.... You can see in day care centres and kindergartens young children working on worksheets or reading from flash cards. You can make children engage in rote counting of large numbers and do exercises reading the calendar. But that doesn't mean you should do it. (Katz, 1988, p.29)

It is possible that commitment to protecting the traditions of early childhood, and the role of play in children's learning, made teachers uneasy about taking a more intellectually focused role with children.

Aligned with this is the question of whether teachers felt confident as purveyors of subject content. Within New Zealand a need for increased teacher content knowledge has been identified (Cullen, 1999; Garbett, 2003; Hedges \& Cullen, 2005). 
Currently, it appears that subject knowledge may be part of the hidden (i.e., underemphasised), or null (i.e., consciously excluded), curriculum offered in early childhood education. It may also be a missing element of early childhood teachers' professional knowledge. (Hedges \& Cullen, 2005, p.17)

Bernstein (2000) distinguished between discourses in types of knowledge. Horizontal discourse is segmented, local, oral, and everyday; and vertical is 'school discourse', hierarchical using specialised language. Here it seemed that vertical discourse was largely absent.

An English study suggested teachers tend to conflate thinking skills with problemsolving (Robson, 2006; Robson \& Hargreaves, 2005) and may not consider their role in supporting children's thinking in detail (Robson \& Hargreaves, 2005). Might the same be true of New Zealand teachers? Children's creative thinking (Robson, 2006), imagination and creativity (Robson \& Hargreaves, 2005) seemed well supported in the centre. They were supported by the provisions for dramatic play, by the supply of open-ended resources and by the relative freedom children had in using these. Children's verbal playfulness and humour, aspects that Alcock (2007) also identified, were further manifestations of their creativity.

In contrast, it seemed critical thinking might be less effectively promoted; the description, in Chapter 3, of strategies Davis-Seaver (2000) adopted to support children's critical thinking provided a useful starting point for considering this issue. Changes Davis-Seaver made in the array and organisation of resources were already largely duplicated in the centre, but were teachers using the other pedagogical strategies she introduced? Teachers here also questioned children when reading books, but did they encourage children to both predict and identify the grounds for their prediction? Often the pressures of mat-times made in-depth questioning unviable; it was more likely to occur in small group situations, but even then pressures of other children wanting books read, impending mealtimes and rostered duties often mitigated against such depth of discussion. When children had conflicts, teachers sometimes adopted the role advocated by DavisSeaver and others (Danby, 1997; Rivalland, 2007) and encouraged children to reach their own solutions through a process of discussion. When physical aggression was an element, they intervened more precipitately with their own solutions, a reaction to conflict others describe (Danby, 1997; Gonzalez-Mena, 1997), which meant on those occasions children were not able to practise reaching their own resolution. Finally, did teachers externalise their own thinking and talk 
about children's thinking? This did happen. A teacher discussed her thoughts about rules needed for the rolled-paper swords before settling on "Being sensible with them, not being scary"; a teacher described to Alex the strategies he was using in doing the alphabet puzzle. But such examples were not frequent. Collectively, these lines of analysis suggested children's critical thinking might be less effectively supported than their creative thinking.

Two further aspects of children's thinking were potential curriculum in the centre. Children invested much time and energy in exploring gendered roles, but were their stereotypes challenged? Children were given many choices in the centre, but did they have opportunities to participate in decisions affecting the whole community? Recognising how strongly some children promoted gendered roles led me to consider whether challenging children's gender stereotypes was null curriculum for teachers. Teachers presented alternative images of being a female with their practical clothing, their behaviour, and their support for Evie who challenged the image of girl-ness accepted by others. Similarly, although they supported boys' gendered positioning through their tolerance of superhero play, they also displayed images of men in nurturing and non-traditional workplace settings. At the time, the teachers' level of support for the differing positions adopted by Evie, Maxine and Grace, and by James and Rex, seemed appropriate. Reflecting on the data since has led me to recognise the depth of gender work taking place, the challenges confronting children who attempted to resist stereotyped roles and the disproportionate amount of teacher time and energy the older boys absorbed. The need for teachers to challenge children's gendered behaviours and thinking has drawn recent attention (Fleer, 1998; Hughes \& MacNaughton, 2001; Keddie, 2003; MacNaughton, 2000, 2001). It is suggested that when teachers' practices are underpinned by a discourse of children as innocent, which signals a response of care and responsiveness, they are unlikely to confront gendered issues of violence and aggression (Grieshaber, 2001; Keddie, 2003). Centre teachers by and large accepted children's gender stereotyping as an expression of their interests and worked to support these. Adopting a more critical approach would have led them to put a higher priority on challenging stereotypes, and the inequities inherent within those (e.g., Ryan \& Ochsner, 1999). In this setting, that deeper level of engagement and challenge to the stereotyped gender work occurring could be said to be null curriculum. 
Recent literature on children's rights (Brandtzæg, 2006; Dalli \& Te One, 2002; Smith \& Taylor, 2000; Taylor, 2000; Te One, 2004, 2005) has highlighted children's potential role in participating in decisions that affect their collective lives. Children were often asked their opinions; they were asked what activities they wanted, which dry clothes they would put on, what song they wanted to sing. Although mat-times, particularly for the older children, were sometimes used as an opportunity for discussion, they were not used as a decision-making forum. Many decisions that affected children were made without their input. Children were not asked their opinions about fixed or rolling morning teas, where trips should go, who could be invited to visit the centre, what new equipment should be purchased, or what they might like to be the focus for their learning. Within the context of New Zealand centres this is unremarkable; in fact these teachers were more proactive in seeking children's opinions than many. However, descriptions of children being part of decision-making (Broström, 2006; Clark, 2004; Paley, 1981; Pramling Samuelsson, 2000) in Chapter 3, section 3.3.5, offer models based on the reconceptualisation of children as thoughtful people (Cannella, 1997; Emilson, 2007; Laevers, 2005; Rinaldi, 2006). The difference between those descriptions and the role centre children were given takes us back to the themes at the heart of the thesis. While much of what happened in the centre was focused on supporting children's social skills of living within a community, the role they were given in decision-making reflects the inequality of their partnership with teachers. Underpinning the genuine warmth and companionship between children and teachers there was, as has been shown in these four chapters, a clear demarcation of power (Boyce, 2001). This is unremarkable; educational institutions are built around such assumptions about the roles of teacher and student. In a critique of contemporary early childhood curriculum, Silin (1997) describes the tension teachers experience between seeing "childhood as a separate life period requiring specialized protections and professionalized care" and as "a time for full participation in the ongoing life of the community" (p.233). The responsibility and pressures of caring for young children, and the traditions of early childhood education inexorably led teachers to focus on the first aspect, and the second consequently received less priority. It seemed children's potential role in participating in decision-making that affected their lives in the centre remained part of the null curriculum. 


\subsubsection{Emotional dimensions that were null curriculum for teachers}

The emotionally warm and positive manner of teachers described by Brennan (2005), and their promotion of "a culture of tenderness" (p.124) were also features of this centre. The converse of this, which Brennan also found, was that teachers rarely expressed negative emotions; this was therefore an aspect of the null curriculum. As suggested in Chapter 3, there are other precedents for this finding; Nuttall (2004) and Norberg (2006) both describe teachers avoiding disagreements with colleagues. Because being warm and responsive with children is a fundamental tenet of early childhood teacher practice in New Zealand, to raise other possibilities seems akin to heresy. Yet the question Woodrow and Fasoli (1998) ask again seems relevant; how beneficial is it for children to grow up with such perpetually unselfish, cheerful female role models?

Were other potential emotional aspects marginalised or excluded by teachers? The following discussion considers the teachers' role in providing children with opportunities to experience excitement and physical risk, and to engage in spiritual and religious experiences.

The excitement older boys generated in their fast-paced chasing games, and their unquenchable enthusiasm for such play, raised the question of whether teachers support activities which allow children to experience excitement, and even risk. For a young child like Robert T., momentary teacher involvement could generate excitement. His delighted reaction to a teacher stepping in front of his bike was described in Chapter 5, and when a teacher initiated spontaneous chasing races to the fence, he participated with excited laughter. Teachers taking an active role in activities that generated excitement with older children was rarer; the single example of a teacher facilitating wild animal play was referred to above. More often it seemed these children generated their own excitement. However, this also raised the allied question of the experience of risk. Given the increasing regulations covering children's safety, were teachers able to find ways that allowed children to experience physical risk, and the feelings of confidence that can come from confronting it (Maynard, 2007; Stephenson, 2003)? Do New Zealand female teachers value opportunities for excitement and challenge as highly as Norwegian teachers in forest kindergartens (mentioned in Chapter 3, section 3.4.3)? There were many moments outdoors when younger children felt at risk. Fleur called for help from the challenge course, Anakin asked for help on 
the triangular climber. For older children there seemed to be fewer opportunities for feeling risk, although Mooloo did retreat without reaching the top of the rope ladder, and James fell off his bike when he was cornering fast. On balance, it seemed excitement and risk were not priorities for teachers, but neither were they null curriculum.

Were spirituality and religion null curriculum for teachers? The exclusion of religion seemed likely given the legal commitment to secular education in New Zealand. However, the Biblical motifs of ark, rainbow and dove on the curtains, the regular karakia (Māori prayer) before lunch, the carol singing, the references to church-going in a teacher-led discussion, plus a mat-time story in which God was a character were all ways in which religious allusions were incorporated into children's centre experiences both overtly and covertly.

The place of spirituality in early childhood education (Bone et al., 2007) and schools (Kennedy \& Duncan, 2006) is an area of professional discussion that is particularly significant in light of the Te whäriki aspiration for children to grow up "healthy in mind, body, and spirit" (Ministry of Education, 1996, p. 9). In the centre, elements of "everyday spirituality" (Bone et al., 2007, p.344) might be seen in the regular Māori prayer, the occasional touch of putting flowers on every table, and less tangibly but more significantly in the depth of emotional rapport between children and teachers. Teachers in Kennedy and Duncan's (2006) study described relationships as a key element in their spirituality, but also felt spirituality could only be at the core of the teacher/child relationship if teachers were aware of, and nurturing, their own spirituality. However, spirituality in this setting was sparsely acknowledged in comparison to Steiner kindergartens (Oldfield, 2001) where moments of group celebration and reflection are woven into the daily, weekly and yearly programme.

\subsection{Extending outwards}

Given that the null curriculum is boundless, there is always more that could be said. The sections above have indicated aspects of curriculum that were potentially present. This section, drawing on a range of sources, suggests other aspects that might have been included in this setting. 
There were content areas and topics that were never included. Conservation was not discussed; children did not take part in recycling or composting. Rhymes were chanted, but poetry was not read. Classical music was not played outside the sleeproom, and there were no activities of focused listening to music. Children moved to music occasionally, but dances were not taught. Board games were rarely played.

Descriptions of children in Scandinavian forest kindergartens roaming in large and sometimes unfenced areas of forest, invisible to teachers, highlighted the supervision requirements New Zealand regulations impose. Were experiences of independence and autonomy null curriculum?

Although teachers encouraged discussions, particularly when older children had a separate mat-time, there were no examples of the kind of philosophical discussions that are described in the literature (MacNaughton \& Williams, 2004). Were there broader issues of social justice and social equity (Robertson, 2006; Silin, 1995) teachers might have raised?

A wealth of cultural and ethnic diversity among children and teachers ensured that, broadly speaking, cultural/ethnic difference was part of curriculum. Puzzles, books and posters carried a range of Māori and Pacific Nations images, and Māori motifs were incorporated into wall displays. Māori and Samoan languages were heard, and used by children in songs. Teachers made occasional references to racial and cultural differences, but was every child's cultural heritage acknowledged?

This section completes this chapter, and also the presentation of the findings. In Chapters 5, 6 and 7 the focus was on the ways in which children experienced, influenced and enacted the boundaries of curriculum. In this chapter, attention shifted to the boundary itself, to what might constitute the null curriculum, and who and how that was decided. Through these four chapters it has been shown that two factors were of particular significance in defining the boundaries of curriculum. The first was the demarcation between children and adults, and the second was that two areas of particular curriculum focus for children were their relationships with others, and the exercising of control/power. The next chapter summarises the arguments that have been presented, considers the implications, and indicates potential areas for future research. 


\section{CHAPTER 9}

\section{COMING FULL CIRCLE}

The wheel is come full circle

(Shakespeare, King Lear)

With this chapter, the thesis comes full circle; here the research questions are revisited and the findings and arguments reviewed. Those findings are then positioned in the broader context of the collective 'conversation', described in Chapter 1, which is emerging among recent New Zealand qualitative theses. Finally, implications for the profession are indicated.

In Chapter 1 a list of aims for the thesis were presented. Throughout this chapter, these aims are revisited in order to assess how effectively they have been met.

\section{Aims for the thesis}

- Provide insight into where the boundaries of curriculum lie

- Describe the process of demarcation, and show how children experience and influence this process

- Within the context of the above, offer insight into how indirect/unintended aspects of curriculum are experienced, influenced and enacted by children

- Provide insight into children's thoughts and feelings about their day-today experiences of centre life

Broader purposes were to:

- Contribute to accumulating research knowledge of curriculum implementation in New Zealand centres in the early $21^{\text {st }}$ century

- Build on and extend the foundations laid by others in exploring strategies for including young children as active participants in research

- Be a strategy of dislocation for others in assisting them to re-examine assumptions about early childhood education. 


\subsection{Revisiting the research questions}

The central research question was:

What are the boundaries of the curriculum in the early childhood setting, and how do young children experience, influence and enact these boundaries?

This was explored through three subsidiary questions:

- How do the hidden aspects of the curriculum, which are implicit in the ways the setting is organised, the kinds of resources provided, and the behaviours of the adults, affect the boundaries of the curriculum that children experience?

- How does the informal and less noticed or unacknowledged learning and teaching that occurs among children affect the boundaries of the curriculum that they experience?

- What potential aspects of learning and teaching, i.e., the null curriculum, are being excluded from the curriculum that young children experience and enact?

To examine the implications of the findings, each of the subsidiary research questions is revisited, before the central research question is addressed. In the process the two central themes that emerged through data analysis are described.

This account must begin by acknowledging that children in the centre were typically engaged, enthusiastic and happy. They were offered a wide range of activities and for much of the day could choose freely among the resources and areas available. Teachers were sensitive and thoughtful in their responses to children's needs and wishes, and they appeared to be motivated by a deep commitment to the children, and to their care and education. Although to a casual observer it might be the freedom with which children moved and chose that was most compelling rather than the ways in which their experiences were limited, it is nevertheless a logical necessity that if there is a curriculum there will by definition be boundaries to that curriculum. This thesis was undertaken in a spirit of curiosity to explore what those boundaries might be, and how children experienced, influenced and enacted them. 


\subsubsection{The adult-child demarcation as a source of curriculum boundaries}

The first research question asked 'How do the 'hidden' aspects of the curriculum, which are embedded in the ways the setting is organised, the kinds of resources provided, and the behaviours of the adults, affect the boundaries of curriculum?' I have argued that the assumed demarcation between adults and children was the most significant source of curriculum boundaries. Although this may seem selfevident, that is in fact characteristic of the hidden curriculum; it is "not something that we must look behind or around in order to detect; in most cases it is plainly in sight, and functions effortlessly" (Gair \& Mullins, 2001, p.23).

Defining children as 'other than' adults is linked to the image of the child as less-less competent, less thoughtful, less skilled; this leads inexorably to the assumption that adults are the decision-makers and children are those for whom decisions need to be made, and that education, together with age, are routes by which children will eventually bridge that generational gap and become competent, thoughtful and skilled older people (Cannella, 1997, 2001; Cannella \& Viruru, 2004; Dahlberg et al., 1999). Defining children as 'other than' adults is also linked to the assumption that children have no role in, or contribution to make to, the 'real world' of adults. These beliefs are deeply embedded within New Zealand society, and can be seen as a 'regime of truth' (McLaren, 2007) which underpins assumptions about children, about education, and about the roles adults and children play as teachers and students. In other times, and in other cultural contexts, different underpinning beliefs result in different relationship patterns between younger and older (Rogoff, 2003). However, the broadest social levels of analysis are beyond the scope of this thesis; rather the concern is with how those wider social discourses about adults and children influence the pedagogy of early childhood education.

The assumed demarcation between adults and children was manifest in the physical and social isolation of the centre, and therefore of children and teachers from the wider community. As Singer (1996) recognises, on the one hand such isolation reflects the child-centred approach, with the inherent valuing of children's education, but on the other hand it results in separation from the mainstream of life. This isolation limited the ways in which teachers could respond to children's interests. For example, teachers recognised children were interested in adults' work, it was a focus in their collective planning, but the 
physical isolation, the staff ratios and the legislated regulations around excursions meant that they were often only able to respond to this interest in peripheral ways. An unanticipated finding was that the adult:child demarcation was reflected within the structure of the centre. It was seen physically in the distinction between adult-only and communal areas, between adult-only resources and those provided for children, and in the differences between the roles which teachers and children took within the centre community. Further, I argued that the ways in which this generational division was embedded within the centre structure and organisation was in tension with, and influenced, the teachers' ability to implement sociocultural practices with children. Centre documentation confirmed teachers' commitment to the principles of Te whäriki, and to working with children in ways that reflected the sociocultural understandings it promotes. But the wider social assumptions about adults and children appeared to counteract these efforts. The demarcation between adults and children was a persistent feature that appeared through much of the data, and was the first emergent theme of the thesis.

A subsidiary finding that arose from detailed consideration of teachers' and children's roles was that the teacher role of educator was largely invisible to children (Stephenson, 2009). This was linked to the finding that older children did not tend to identify themselves as learners in this setting; when they identified hard things they were learning they tended to be out-of-centre experiences such as learning to write. Conversations with children as they shared their portfolios also generated very few references to learning.

There were incidents where the teacher-child demarcation was blurred and these offer kernels of opportunity for future change. Trips out of the centre and visitors coming in diminished the physical and social isolation. When teacher/s and children shared moments of genuine emotion together, as in the ritual of farewelling Mooloo, there was a sense of authenticity and shared experience in which age was irrelevant. When teacher/s and children engaged together on tasks, there was an opportunity to work in a more collaborative way. This seemed particularly significant when it was defined as a meaningful task, 'real' to both adults and children, such as building the house-frame. Involving children in regular tasks which teachers habitually performed, such as patting children to sleep, further blurred the assumption that children played while teachers did the real work. Some tasks also offered children access to resources defined as 'adult' 
in the centre — the knives for spreading biscuits, the battery-powered drill—and this provided a further way in which the generational distinction was diminished.

These findings demonstrate how the aim of providing insight into where the curriculum boundaries lay has been met within the thesis. They highlight the difficulties that we in the profession continue to face in making fundamental changes in the way we envision children and teachers. Such changes are made more challenging because familiar attitudes are constantly reconfirmed by the visible physical dependence and lack of verbal expertise of the youngest children. This thesis has underlined the difficulty of attempting to unwind habitual ways of thinking and to blur the categories of 'adult' and 'child' in the work of early childhood education.

\subsubsection{How did the learning and teaching among children affect the curriculum boundaries?}

The second subsidiary question asked 'How does the informal and less noticed or unacknowledged learning and teaching that occurs among children affect the boundaries of the curriculum?' A first step towards answering this was the finding that relationships with others were at the heart of curriculum for children, and that this was the matrix within which children developed their identity within the centre. A second step came through finding that a dialectic quality characterised many of the relationships within the centre; on the one hand there was a positive focus on establishing relationships with others and becoming/being part of the community, and on the other hand there was a concern with exercising control/power within those relationships. It was argued that every child appeared to be exploring the construct of relationships with others, peers and teachers, and becoming a part of the community, as part of a larger project of establishing/ defining their identity within the centre. While it was not clear that every child was exploring issues of exercising control/power, many clearly were, both with teachers and peers. The fact that some children exercised control/power in overt ways and in public forums such as mat-times ensured issues of power were a significant aspect of curriculum for all children, whether as a wielder, a recipient, or a witness. Although teachers, who held the balance of power, characteristically used positive approaches in gaining children's compliance, the exercising of control was a frequent, albeit often masked, aspect of their interactions with children. The finding that power was a pervasive and interwoven feature of 
relationships was unexpected, but reflects Gore's (1992) redirection of attention from the dualities of dominant and subordinate to "the exercise of power at the micro levels" (p.61).

Having identified these two elements-a positive focus on establishing relationships and becoming/being part of the community, and a subsidiary concern with exercising control/power within those relationships-as persistent threads intertwined in very many of the interactions both between teachers and children, and among children, I argued that these were central concerns of children, and therefore curriculum content which they introduced. Without children's concern with the exercising of control/power, teachers' preferred strategies would have ensured issues of power remained covert. As analysis of rules showed it was only when children were, wittingly or unwittingly, noncompliant to adults, that issues of control/power became overt. The recognition that these two elements were curriculum concerns for children was identified as the second emergent theme of the thesis. The teacher-child demarcation was an integral element within this theme.

It was argued that for children, resisting teachers served two different purposes. First, children often resisted teachers for their own purposes of reaffirming their position and status within their peer group as others (Alcock, 2005, 2007; Brennan, 2005; Corsaro, 1985, 1997, 2003; Kyratzis, 2004; McCadden, 1998) have shown, although it was found here that the reference group was typically a subgroup rather than the wider group of all peers. Such acts of defiance were typically short-term acts in front of peers, and often suggested peers' approval was of more immediate significance than teachers' disapproval. Secondly, it was argued that children's resistance at times allowed them to exercise some control within the adult-dominated world. Individual acts of resistance, private interactions between a teacher and child, provided the clearest examples and teachers' responses showed they recognised children's resistance as a gesture towards negotiation, and typically responded to this favourably. While the power imbalance remained with the teacher, who typically proposed the compromise, and who held the right to grant/refuse, children learned that resistance gave them some leverage, and the potential for negotiation existed. 
There were also other, more obvious ways in which children influenced the scope of curriculum. A central one was their interest in defining themselves by gender. Stereotyped images of what it is to be female and male were promoted by children, with only a few appearing to resist those roles. With hindsight, I recognise that for many of the older children, this was a third avenue through which they were exploring and establishing their identity within the centre, along with building relationships and exercising power/control. Indeed, the exercising of power/control was an integral aspect of the gendered role some older boys were adopting. However, for younger children the interest in gender was less evident and so it was not identified as part of that second emergent theme.

Finally, individual children brought their own interests into the centre. When the environment, physical and social, was conducive to the expression of these, the funds of knowledge (Hedges, 2007) children brought enlarged the curriculum for their peers. Even in the absence of real horses, Cassidy's knowledge about horses was shared with others in play with the small plastic horses, and in sharing books about horses.

\subsubsection{What was null curriculum?}

What potential aspects of learning and teaching, i.e., null curriculum, were excluded from the curriculum that young children experienced and enacted? In asking this question, the thesis has raised issues that are infrequently addressed; indeed no research with a focus on the boundaries of curriculum in early childhood education was located. In preparation for the research, a review of relevant literature was undertaken, and this may itself prove useful to others.

In introducing this area of findings, I acknowledged that the nature of the question meant data were less substantial. However, it was clear that, although there was broad consensus about what was null curriculum, there were also differences between teachers' and children's definitions. For example, the body appeared to be null curriculum for all, but the ways in which children used some language associated with the body, including sexual allusions, indicated they considered it to be null curriculum for teachers. Individuals' definitions of what constituted null curriculum for them personally reflected the identity they had established within the centre. For example, playing with dolls appeared to be null curriculum for James. It was argued that children accepted some boundaries, such as the 
invisibility of the body, but actively resisted others. Two common forms of resistance were bringing items from home and using 'disapproved' language. A number of preconceptions about what might be null curriculum for teachers were overturned: religion, popular culture and teaching reading were all, at least on occasion, part of the centre programme. Other aspects of curriculum that children introduced, such as superhero play, appeared to be borderline curriculum for teachers, tolerated but rarely actively extended. Undertaking analysis from this perspective reinforced earlier findings and showed that the wider world of adults was by and large null curriculum, despite teachers' efforts to bridge this gap. In describing what intellectual processes might be null curriculum, the discussion referred particularly to children's critical thinking, considered whether their acceptance of gendered stereotypes might be challenged, and suggested the role children might play as decision-makers within the centre. In considering what emotional dimensions might be null curriculum, the habitually positive persona of teachers, the opportunities for children to experience risk, and the place of religion and spirituality within the programme were discussed.

\subsubsection{What are the boundaries of curriculum, and how do young children experience, influence and enact these?}

In reviewing the findings in relation to the subsidiary questions, the answer to the overall research question has been summarised. However, alongside the specific findings revisited above, other arguments emerged which hinge around the understanding of curriculum as complex (Haggerty, 1998, 2003).

Recognising how greatly each child's experience of curriculum may vary was the first way in which the complexity of curriculum was demonstrated. Each child is likely to have a very different experience of curriculum, even on a single day in a single setting. Children move individually, in subgroups, and even when they come together they may have entirely different experiences of a collective episode such as mat-time. It is likely that taking a teacher-centric viewpoint often leads to the range of variation in children's experience being overlooked. James (2004) warns against using the 'child' in talking about children's collective experience; I am wary that the habitual use of 'curriculum' as a singular implies all children collectively share the same experiences. This thesis demonstrates how untrue that image is. 
The second way in which the complexity of curriculum was demonstrated was through recognising the complexity of children's social lives. Singer (1996) writes that children's "interest in one another and the value of these contacts are often underestimated" (p.36); the episodes described here indicate that the difficulties involved in forming and sustaining peer relationships might also be an underestimated area of curriculum. Conversations with parents and children confirmed observational data that suggested how challenging learning the social intricacies of relationships with others could be (Corsaro, 2003; McCadden, 1998).

To return to the aims this thesis set itself, how effectively do these findings and arguments describe the boundaries of curriculum and the process of demarcation? How effectively do they show children's role in that process? How much insight do they offer into how indirect/unintended aspects of curriculum might be experienced, influenced and enacted by children? While it is the nature of qualitative research to produce findings that are indicative rather than definitive, this thesis has generated a number of insights into how the unplanned and less obvious processes of doing and defining curriculum occur within a centre, and has described ways in which children influence these processes. Given the broad scope of the question, and the under-researched nature of the topic, it is not surprising that questions still remain. However, the fact that many aspects of the themes and findings were consistent threads within the analysis, regardless of the perspective adopted, is an indication of their inherent robustness. (These questions are addressed again in section 9.3.)

\subsection{Taking unfamiliar perspectives}

In drawing the thesis to a close it is appropriate to reflect on how the theoretical framework and strategies of dislocation have contributed to the investigation. The central thrust that underpinned the selection of the theoretical framework, the methodology and the use of sensitising concepts was that each required me to step outside my assumptions and expectations, and take different and unfamiliar perspectives.

\subsubsection{The theoretical framework}

The theoretical framework selected combined the theoretical perspectives of critical pedagogy with the philosophical understanding of children's roles from 
the sociology of childhood. Neither of these were familiar approaches. Critical pedagogy was the context in which the research questions were framed, and it was the source of literature and research on the constructs of the hidden and null curricula. Although a focus on the assumptions and power differentials that underpin early childhood education may seem alien to those within the profession, others have indicated its value (Keesing-Styles, 2002; Kilderry, 2004).

"Schooling, even nursery schooling, is one of the central ways that society organizes power and influence. Recognizing this means that early education should not be exempt from a more political analysis of its program" (Silin, 1995, p.105). Brandtzæg (2006) has highlighted how a discourse of empowerment can distract from wider dimensions of power, that we convince ourselves "that a child who can decide how to dress or what to eat, is 'performing power'; even though that same child is sent to a day care institution every day" (p. 91). At the level of the educational institution, the framework of critical pedagogy assisted in identifying the ways in which the apparently benevolent discourses of early childhood education overlaid a deeper discourse which positioned children as other than adults. This in turn helped me to understand the ways in which this deeper discourse was influencing and restricting the structures and practices of early childhood education, and was therefore a central source of boundaries to the potential scope of curriculum. But critical pedagogy was also useful in understanding the significance of issues of power and control at a micro-level within the setting. Gore (1993) challenged critical pedagogy for perpetuating "a simplistic dichotomy between empowerment and oppression" (p.59) and argued for a shift towards "a problem of multiplicity and contradiction" (p.61). Recent writing by McLaren (2007), influenced by the work of Foucault, was useful in understanding power as interwoven through relationships. Therefore, at both the macro- and micro-levels, critical pedagogy offered a framework for understanding issues of power and control within and beyond the centre.

However, there were areas where my assumptions and experience blinkered me. Kincheloe and McLaren (2005) warn that "oppression has many faces and that focusing on only one at the expense of others (e.g., class oppression versus racism), often elides the interconnections among them" (p. 304). They also argue that "mainstream research practices are generally, although most often unwittingly, implicated in the reproductions of systems of class, race, and gender oppression (Kincheloe \& Steinberg, 1997)” (Kincheloe \& McLaren, 2005, p.304). 
I am aware that racial issues were not prominent in my analysis, and yet there were two occasions when I wondered if there was an element of racial discrimination within an interaction, which makes me conscious of how much I suspect I missed. I would see this as a limitation of the thesis.

The second and intersecting framework used within the thesis was the sociology of childhood. This was important initially as a source of research strategies in supporting the methodological focus on young children. However, it gained additional significance with the identification of the first emergent theme, the influence of the adult-child demarcation. Further reading in this area reinforced the importance of generational issues in understanding children's lives (Mayall, 2000) and underlined the complexity of children's positioning in the modern world (Mayall, 2002; Prout, 2005; Wyness, 2006).

There was unexpected resonance between the two frameworks. Both contributed to my theoretical understanding of the significance of the demarcation between teachers and children that was identified. Critical pedagogy offered a way of understanding it within the field of educational institutions, and the sociology of childhood showed it to be a reflection of a wider social phenomenon. Both contributed to the microanalysis of the relationships between teachers and children within the setting, and to my growing understanding of how wider social discourses were embedded within the structure and organisation of the centre. In turn, this indicated why implementing the sociocultural practices inherent in Te whăriki might be so difficult; both critical pedagogy and the sociology of childhood highlighted the issues of power inherent within the assumed adult-child demarcation which were in tension with sociocultural approaches. Siting the thesis at the point of intersection between the two frameworks proved to have been a robust option.

Other theoretical frameworks could have been used. Activity theory was originally considered as a third dimension, alongside critical pedagogy and sociology of childhood, but the complexity of managing a three-theory framework did not appear to be warranted by the additional benefits it would have brought. Instead it was decided to use the three elements from activity theory as sensitising concepts; this is discussed below. Foucauldian concepts appeared increasingly pertinent as the theme of power and control emerged during data analysis. However, the framework of critical pedagogy offered a focus on issues of power specifically within educational 
contexts which meshed with the thesis topic. Because critical pedagogues have increasingly acknowledged Foucault's work (Kincheloe, 2004; McLaren, 2007) it was possible to incorporate concepts such as the regime of truth, and the notion of power not as possessed, but rather as something which is exercised and interwoven into relationships, without changing the theoretical framework.

\subsubsection{The strategies of dislocation}

Strategies of dislocation (introduced in Chapter 2, section 2.4) were not a concept that I had met in the literature. They were strategies that were created for the purposes of this thesis, to assist in seeing the structures and practices of early childhood education with untrammelled eyes. Each required taking an unfamiliar perspective to consider a very familiar context. The first was a focus on what constituted the hidden and null curricula. The second was foregrounding the perspectives of children. The third was the use of the three concepts from activity theory - the physical resources, the roles, and the routines, rituals and rules-as sensitising concepts (Bowen, 2006) in the analysis and as a focus in writing Chapters 5,6 and 7. Although weaving these elements together was at times conceptually challenging, collectively they constantly reinforced the requirement that I step beyond my assumptions. Although each perspective revealed new aspects, the central themes of the thesis were affirmed in each. For example, while the second theme of the thesis was not introduced until Chapter 6, the description of the teachers' control of the environment, and of how children resisted this at times, was an early indication of the significance of issues of control/power within the setting. One indication of the success of adopting these strategies is that the findings generated were both unexpected, and somewhat uncomfortable.

\subsubsection{The focus on children's voices}

Increasingly research in early childhood education includes children as participants and informants (e.g., Einarsdottir, 2005, 2007; Greenfield, 2004, 2007; Hedges, 2007; Smith et al., 2005), and one of the aims for this thesis was to 'Build on and extend the foundations laid by others in exploring strategies for including young children as active participants in research'. This section describes how each of the child-focused strategies (introduced in section 4.4.3) was used and offers insights that might assist other researchers. 


\section{Strategy 1: What children call this place}

The folder of photographs of sites within the centre was designed for initial gettingto-know-you conversations during the first days in the centre, with a subsidiary focus of discovering the names children used for places. A number of children participated, but only six were identified, as I was still learning names. Even very young children were interested. Information was recorded in notes during and immediately following conversations, as I was reluctant to audio-tape during those first days of relationship-building. The interactions alerted me to the importance of 'stepping back' in my listening. For example, I learnt the significance of bagsCharlie volunteered who owned three of the bags showing in the locker room photo, Sina knew who owned the yellow pack visible above the changing table.

\section{Strategy 2: Child-led photo tour of the centre}

Child-led photo tours were initially planned as a way of exploring children's thoughts about the environment. They began with a request to show me the places they liked, their favourite places. Thirteen children chose to do photo-tours (seven girls and six boys, aged 2,3, and 4), three undertook more than one, and one boy did three tours. Three children (aged 2 and 3 ) requested I take all the photographs, some children elected that I take some so that they could appear, but for many older children being in control of the camera appeared to be an attraction. Two-year-olds enjoyed the chance to see themselves immediately by pushing the 'quick view'.

This strategy generated information about children's preferences. Tallying the photographs showed just under a third were of places or of objects with no person, or only peripheral/distant figures. The other two-thirds contained people, with equal proportions showing the child leading the tour, obvious people in a centre context, and close-ups of people. The most photographed activities/places were books, bikes, general views of the playground, swings, and the fort structure. Although most children chose to move outside to take their first photographs, and the majority of individual tours contained more outdoor than indoor shots, collectively there were more indoor photographs. This anomaly reflects the number of indoor photographs James took, and Evie's decision to take a large number of photographs of her portfolio as part of her photo tour.

The tours also opened up a much wider range of data. The significance for Grace of her friendship with Maxine was indicated by her allowing Maxine's choices to 
guide her own selection of sites, and by her request to know "What did Maxie say?" when asked why the tunnel was a special place. Rex's home-based interest in electronic equipment was evident in his enthusiasm for, and expertise in using the camera. The significance of people was underlined by the frequency with which children took photographs of others, although this modification of the process by children may in part be explained by the emphasis on people in customary family snapshots and in centre documentation. Restricting the number of photographs and/or providing a clearer rationale, (for example, collecting ideas for a new playground design (Clark, 2007)), might have led to more easily quantifiable data.

A selection of photographs from each tour was printed and returned to the child. Initially it was intended to print every photograph, and for many children this occurred, but sometimes the number taken made this impractical. Using the digital camera meant, however, that a full record of each child's photographs could be preserved. Returning the folder of photographs to the child provided further opportunity for discussion about why some places were special, but sometimes also led to quite different data. It was when Mooloo was talking about her photographs that she told me she didn't like it "when James hurts me". While some children appeared to treasure the folder-Sina carried it with her all morning - my impression was most children valued the process of taking photographs more than the photographs themselves.

\section{Strategy 3: Photos and stickers>photo chart and counters}

This evolving strategy was designed to explore children's reactions to centre activities/events. Fourteen children undertook the activity, two of whom completed it twice, and one did it three times. They ranged in age from 2 years (two girls) to 4 years (five girls and two boys), and even younger children delighted in the container and counters. Occasionally this activity was done with one or two children in a retreat room, but more often in the main room surrounded by other activity. This had the advantage of engaging other children, which could become a disadvantage if they were impatient for a turn. Converting the photographs to a chart meant less space was required, and the introduction of happy and sad counters introduced a clearer element of decision-making. Children enjoyed the novelty of equipment, and being in charge of the process. Sometimes it was clear children understood the happy/sad distinction-Mulan ( 2 years 3 months) placed counters "slowly and thoughtfully", often using naming the places and used only two sad 
faces. Other times I was less sure-Evie's concern appeared to be to include every photo. Using a tape recorder to capture interactions was useful, and I named photos as children placed counters which provided an audio record of the order of their choices. Taking a photograph of the completed chart/photographs was a quick way of recording the placements before another child began. Because a majority of photographs were of indoor activities/events, it was difficult to draw conclusions about indoor/outdoor preferences. I was also aware the quality of the photograph probably influenced children's selections; for example, a distant photograph of the fort was not selected by any child, and yet the fort was frequently photographed during tours. The most popular activities/events were the Babysafe, the display of books and soft toys, the dress-ups, the school-lunch gathering, and the car in the barkchips. Only girls positively selected pictures of the challenge course, carpentry, and painting and drawing. Only boys positively selected the picture of the collage area. There was no clear pattern in the use of the sad/dislike category, except that most children chose not to use it; only 14 of the 169 choices made were sad/dislike.

Again, the interactions often provided more interesting insights than the tallied choices. The photographs were chosen as depicting activities/events but children often re-directed my attention back to the significance of people-looking for themselves, identifying others. It was after completing this activity that Maxine told me Grace laughed at her (described in 6.3.1). Cassidy's response to being asked to make a selection highlighted how my assumptions constrained the scope of children's responses. He circumvented the absence of horses in the photographs by placing a plastic horse on the photograph of the sandpit and putting all his stickers there. Had I insisted on my intention of one sticker per photograph I would not have understood his meaning (Stephenson, 2009a). When a child repeated the activity, their selections changed-Rex did it twice within a week, duplicated seven of his original 24 choices, added three new ones, and put two sad faces on what had been positive choices. This was a useful reminder of the danger of overestimating the significance of any individual response.

\section{Strategy 4: Picture questionnaire}

The picture questionnaire-a 4-page document, 2 questions per page, with space below each-was introduced to offer children a chance to respond through 
drawing, and was intended to explore both their relationships with peers, and their perceptions about their own learning. The questions were:

- Things I like to do at centre are

- People I like to play with are

- People I don't usually like to play with are

- These are things that I am really good at doing

- These are things that I still find hard to do

- Things that I have learned while I have been at centre are

- I have shown other kids how to

- Things that I like to do at home.

Reluctance to initiate discussion of disliked peers made me hesitant to introduce this; it was used with only four children, three girls aged 4, one boy aged 3 . Two girls worked alongside each other, the others were presented with it individually in a withdrawal room. Audio-taping the interactions was valuable, although Sina's interest in the tape exceeded her interest in drawing, and I could have dispensed with the artwork. No one completed the questionnaire; four questions would have been a more appropriate length.

\section{Strategy 5: Sharing learning portfolios}

Sixteen children (seven boys and nine girls, aged between 2 and 4) either offered or agreed to show me their portfolios, and three showed me theirs three times. The strategy of using a camera to photograph favourite pages was introduced after the first children approached were unenthusiastic. The disadvantage of using the camera was that interest in photographing at times took precedence over the portfolio-I even had to turn the pages for Aidy. Sharing portfolios was almost always done in the quiet room where the portfolios were stored, often with other children watching, commenting and/or waiting for a turn. Whenever possible the interactions were audio-taped, and the transcripts of the conversations plus the children's photographs provided detailed data. This strategy might have generated more conversations about learning if revisiting portfolios had already been a frequent centre event, as it has since become.

\section{Strategy 6: The New Kid book}

The book was created using clip-art, but also included a photograph of all the teachers and children as a reminder to children of who might be potential teachers 
(see section 4.4.3 for the storyline). The new kid was a genderless figure to give children freedom in identifying and naming her/him. The intention was that a child would name the new kid, answer the printed questions about who would teach her/him what, and receive their own version of the book, containing their responses. However, using the book showed it did not hold children's attention, and at least one child was confused by the fact the new kid's appearance changed. (This was then amended.) It was used eight times, with boys and girls aged from nearly 3 to 4 . Although it prompted some interesting comments around learning/teaching, it was not a strategy that children requested; I suspect more professional illustrations and production would have increased its appeal.

\section{Strategy 7: The Naughty book}

This book had a similar format and suffered from the same problems regarding quality of presentation (see section 4.4.3 for the storyline). Children seemed to struggle to think of 'naughty things' a child might do in the centre, and so it was used only five times with boys and girls aged from nearly 3 to 4 .

\section{Strategy 8: Informal conversations}

These were a constant-almost always when I was present in the communal areas, children chose to interact, keen to have access to an adult who had no other duties and was interested in spending time with them. Many conversations related to the immediacy of what the child/children were doing, much was not audio-recorded, although I typically jotted down details which I expanded on later. I re-learned how many insights came from spending time with a child, talking within the context of their play (Stephenson, 2009b) and the benefits of a prolonged datageneration period (Stephenson, 2009a). On each visit I attempted to spend some time without any equipment, interacting with children, as they wanted, as reciprocation for the time they spent with me. Retrospective notes were often made of aspects of these interactions.

\section{Strategy 9: Observations}

As described in 4.4.3, observations were the matrix. While they lack the panache of more child-focused strategies, observations were a very significant source of data, and often provided a context which helped me to comprehend children's responses. Angus's decision to put a sad face on the sleeproom would have seemed less significant if I had not observed his struggles; I never knew why he 
also put one on the zoom slide. Cassidy's decision to place a plastic horse on a photo of the sandpit, and put all his stickers there made sense when I reflected that in 12 of the 28 observed episodes which included him, he had initiated references to horses.

I believe this thesis has built on and consolidated what others have done in a number of ways. Firstly, a point of difference with much earlier research is that every child attending the centre was a potential participant. (Although six children were identified as focus children towards the end of data generation, the most significant difference in data collection for these was an interview with a parent; to the end all participants were important.) This was partly a matter of equity; all children were offered access to whatever privileges they might consider involvement in the research had to offer. But it was also a response to MacNaughton's (2003a) question, cited in Chapter 4 "how will I honour those children whose voices struggle to be heard?" (p.41). I was wary any process of pre-selection might highlight children whose voices were already dominant. It was fortuitous that parents' reactions overturned my initial plan to include only children aged 2 and over. With hindsight, not to include all children on agerelated grounds would have sat uneasily with the broader aims of this thesis. While no data in response to the child-focused strategies was recorded from children aged under 1 at the start of data-generation, they shared ideas effectively in non-verbal ways-for example, Macauley's determination to get outside.

Secondly, this thesis demonstrates the benefits of spending a prolonged time in the setting (Corsaro, 1985, 2000; Hadley \& Nenga, 2004; McCadden, 1998; Paley, 1984, 1986, 1990). This meant there was time for relationships to develop and for children to approach me when and how they chose. It meant there was a chance for all children to choose to engage in the research strategies and to repeat them if they wished over the months. And whenever a child repeated a process, there were inevitably changes in their responses which I believe contributed additional depth and complexity to their original response. This suggested how much the immediate 'here' and 'now' influences a child's (or an adult's) responses, and how precarious it could be to base conclusions on single research interactions. It was only with this deeper contextual knowledge that possible implications of children's comments were recognised, yet I always knew there were others the teachers would have understood. While there are inevitable 
weaknesses in using a single setting for research, it seemed the benefits of prolonged involvement in the setting outweighed these.

Thirdly, this thesis has continued the recent focus on finding ways to include children as actively as possible. This was seen in the care taken to make the assent process for children authentic, and in the range of strategies used in talking with them. The child-focused strategies were devised within the context which allowed me to use what I found children enjoyed, while at the same time following up successive research hunches. It became clear children enjoyed strategies that maximised their control, and the digital camera was particularly important here, especially for older children. As Clark and Moss (2001) found, using a range of strategies was effective because children could select the activity they preferred. However, taking a respectful approach meant I was also aware that children often preferred to spend time with me, without the focus of the research strategies.

Fourthly, I learned the importance of 'stepping back'. Stepping back from my preconceived plans, and allowing children to guide the process (for example, not insisting that they only photograph places), freed them to respond in unexpected ways. Stepping back from the data (Stephenson, 2009a) allowed often unanticipated messages to be heard. With a more specific research question, or one which was more easily explained to children, such 'stepping back' might have been less necessary.

The tools themselves were important. The digital camera was invaluable for recording visual data quickly which could then be downloaded, coded and revisited, and it was also useful for recording written records which could later be transcribed. Having a colour printer meant children's photographs could be printed and returned on the next visit. The tape recorder was important for recording interactions and allowing children to revisit them.

This thesis has led me to reconsider the emphasis within research on children's perspectives. I introduced children's perspectives (in 1.1) as including both children's knowledge (listening to and hearing what children say) and taking the perspective of the child (observing and interacting with them, and on that basis imagining and identifying with how they think and feel). While the former is increasingly and rightly gaining prominence, I argue for the value of marrying the 
two approaches together. The data gathered here using both approaches provided greater depth of understanding and insight into children's thoughts and feelings about their day-to-day experience than either could have in isolation.

Finally, a comment written after a challenging session of looking at children's portfolios should dispel any illusion that data generation was an untroubled process:

Issues persuading them to take turns, Amanda finding my cards and unpacking them, Aidy finding us and coming in and running a tractor up and down the back of my head making loud noises, Cassidy wanting company ... Maxine losing interest, and consequently Grace losing interest, [a teacher] doing a drumming session immediately outside the door, the tape running out (and I thought the batteries were dead so didn't turn it over) etc, etc. [29/8/05]

\section{3 In the context of other recent New Zealand theses}

Qualitative research is, by nature, a complex process that reveals multiple realities and truths. While the generic inductive qualitative approach was used to ensure that emerging themes and categories were grounded in the data and therefore credible, the findings cannot be replicated because no other study will ever engage with the same aspects of reality. Cullen (2003b) draws attention to the limitations of small-scale qualitative studies such as this, and Graue and Walsh (1998) suggest "much interpretive work falls short through failure to connect to the larger context" (p.10). One possible response to these critiques is to compare the findings from this thesis, with those from other recent qualitative studies completed in similar New Zealand settings. (These theses were introduced in Chapter 1, section 1.2.) Collectively, these have provided an unprecedented depth of insight into current practices, and links to these have been profiled throughout. The further step is now taken of considering the conclusions from this thesis within the context of this collective conversation, and identifying areas of alignment and difference; this provides a dimension of external triangulation. While this pool of research is limited, each researcher has taken a different focus, drawn on different areas of international research, and used different theoretical frameworks and methodologies; collectively these differences contribute to the significance of identified common themes. The fact that the data for this thesis were collected in 2005, and the initial analysis was completed before I read the theses by Alcock (2005), Brennan (2005), Bernstone (2007), and Hedges (2007), increases the significance of the areas of alignment in the findings. The following discussion will therefore demonstrate how this thesis has met the broader purpose 
of contributing 'to accumulating research knowledge of curriculum

implementation in New Zealand centres in the early $21^{\text {st }}$ century' and in particular to the emerging collective conversation among the recent qualitative theses.

\subsubsection{Alignment of findings}

Many points of alignment emerged. Some were points of detail:

- A recognition of mealtimes as a significant forum for consolidating children's peer relationships and shared peer culture (Alcock, 2005)

- the importance of shared rituals such as birthdays in reinforcing children's sense of belonging (Brennan, 2005)

- teachers' differential engagement with children's interests (Hedges, 2007).

But there were more significant areas of alignment and difference that were not always so overt.

One less obvious area of alignment is the growing confidence to critique and challenge, which has made it easier for each successive scholar to take a critical stance, not as a criticism of individual settings, but rather for the insights it may offer the profession. As Giroux (2001) warns, in the context of the hidden curriculum, analyses will "gain some theoretical mileage only when they move from description to critique" (p. 47) and address broader underlying issues. The culture of critique others had established made it easier for me to select a topic that would potentially challenge aspects of practice and tradition, and to present critical findings and less benevolent images of centre life.

I have argued that there are unresolved tensions for teachers in the interplay between wider social assumptions about the roles of adults and children and their commitment to implementing socioculturally oriented practices with children. Whereas Brennan (2005) relates teachers' struggle to implement sociocultural understandings to the structural arrangements, I argue that at a deeper level, those arrangements are a reflection of wider social assumptions around adults and children. In arguing this, I am referencing earlier contributions from McLeod (2002) and Nuttall (2004). McLeod (2002) shows the significance that differing constructions of the child have for leadership and management practices, and yet how rarely those underpinning images of the child are consciously recognised, 
acknowledged or discussed in centres; she recognises this can lead to a lack of congruence between philosophy, centre structures and centre activities. Nuttall (2004) identifies how pressures of work and a prioritising of collegiality can lead teachers to side-step tensions and ambiguities in their beliefs and practices; she describes teachers glossing over the differences in their notions of curriculum, and discrepancies between these and their enactment of curriculum. Collectively, these findings suggest misalignment between professed beliefs and the reality of practice continues to be a concern.

I have argued firstly that the structures of early childhood education settings isolate children and teachers from the wider community, and secondly that the demarcation between adults and children is embodied within the architecture and organisation of the centre itself. Brennan (2005) also reaches the first of these conclusions, and her findings lead her to question the structural arrangements which separate children and teachers from the wider community. Hedges (2007), acknowledging Brennan (2005), also recognises the limited opportunities for children to be part of adult settings, and proposes that "the physical structure and activities of an early childhood centre could be reviewed to be less institutional and separate from the adult world" (p.271). In contrast, Wright's study of two home-based settings showed that "moving out of the home and into the community was a significant and integral feature of the environment" (p.176). There is, therefore, a growing strand of critique which questions the traditional physical and social isolation of early childhood education centres. None of these scholars has directly addressed the second part of the argument that was presented here; that the demarcation between children and adults was also embedded within the centre structure and organisation, although aspects of findings from both Brennan (2005) and Hedges (2007) provide some peripheral support.

I have argued that offering children more opportunities to engage collaboratively with teachers in tasks that are meaningful within the centre community is one strategy for blurring the teacher-child demarcation. Along similar lines, Brennan (2005) comments on the authenticity of moments when teachers and children were engaged in 'real life' interactions and conversations (p.210). Hedges (2007) suggests teachers could engage publicly in relevant work activities that are likely to be of interest to children such as using a sewing machine, marking the roll, writing shopping lists, or buying equipment. Wright's (2003) description of 
children's participation in a range of authentic tasks that are more frequently available in home-based settings suggests the satisfaction children may derive from such opportunities.

This thesis has underlined the extent of the power imbalance between teachers and children and argued it is masked by wider social assumptions about the positioning of adults and children, by teachers' use of positive strategies to gain compliance, and by traditional early childhood education discourses of empowerment and choice. Others have approached the issue of power peripherally. Both Jordan (2003) and Bernstone (2007) have drawn attention to implicit power-orientations in teaching strategies, and highlighted difficulties teachers seem to face in shifting their practices to include those such as co-construction and negotiation which allow children a more equal role. The finding in this thesis that teachers avoided overt displays of authority, and habitually used positive strategies affirms findings from Brennan (2005). Although only early results are available from Te One's investigation of how teachers and children perceive children's rights in early childhood settings (Te One, 2004, 2005), and how these rights are enacted, her work is likely to provide further insight relating to power dynamics.

A second dimension of power investigated in this thesis relates to children's responses to teacher-derived rules, and the findings build on arguments proposed by both Alcock (2005) and Brennan (2005). While children's resistance to rules was not a central focus for any of the three studies, it nevertheless emerges as a significant aspect of findings in each. The preponderance of rules, noted in this thesis, was a feature Alcock (2005) also comments on. More significantly children's resistance and the reasons for it were themes that emerged for both Alcock (2005) and Brennan (2005). Alcock (2005) observed many examples of children's playful subversion of rules, which she sees as a process by which children adjust to those rules, and to notions of rules and rule-breaking in general. However, she also recognises the importance of resistance for children's developing sense of peer agency. Brennan (2005), with a focus on enculturation, concludes children's resistance provides opportunities for teachers to clarify, and to adjust the fit between individual desire and group expectations. So, more strongly than Alcock (2005), she positions resistance to rules as a potential avenue for change within the community. The arguments proposed in this thesis offer support for both perspectives. The finding that resistance was a way in which 
centre children reconfirmed their peer status and membership aligns with Alcock's finding regarding peer group agency. There was also support for Brennan's finding that resistance may be linked to change, although our lines of reasoning differed. I argued children's resistance, particularly when expressed in one-to-one interactions with a teacher, was often an implicit request for negotiation, which teachers frequently acknowledged by making a compromise. Therefore, while my argument focused on the individual and Brennan's focused on the system-wide function of resistance, the potential for change through resistance was common to both.

I have argued relationships were the core of curriculum. Alcock (2005) also highlights the significance of peer relationships, suggesting that the focus on individuals means these are often overlooked. Similarly, Brennan (2005) found that "children's need to affiliate with others presented as the basis of most interactions" (p.209), and argues "relationships were the essence and the basis of enculturation" (p.205). However, in keeping with her research focus on enculturation, her interest was more particularly on teacher-child relationships, and indeed she proposes 'teacher-and-child' as the unit of analysis for sociocultural research. My findings suggest this over-prioritises the role of the teacher, and underestimates the importance of peer interactions.

Both Hedges (2007) and Brennan (2005) argue for recognition that children are interested in complex issues. Hedges (2007) believes children's interests need to be interpreted in ways that allow "meaning-seeking about deep and serious issues of citizenship, culture and identity" (p.268) to be included. The argument in this thesis that issues of relationships with others, the exercising of control/power, and often also gender, were constructs children were using in establishing their identities, and were central curriculum concerns, provides support for Hedges.

At a more fundamental level, there is considerable alignment between the identified themes in Brennan's work and in this thesis. Brennan (2005) notes that the themes of "relationships and relating to others" and "agency and resistance to participation or acceptance of social suggestions" (p.107) are thematic elements that appear repeatedly through her data. This provides unexpected resonance with the second central theme of this thesis - that establishing relationships and exercising control/power were central curriculum interests for children. However, 
Brennan adds a third element, "tools and tool use" (p.107), which was not a feature of the current analysis. Unexpectedly, Nuttall's (2004) findings also provide a point of resonance with this theme, in relation to the teachers. Drawing on the work of Wenger (1998) she introduces the two processes of identification and negotiability; identification is the process of belonging within a community, and negotiability is "the ability, facility, and legitimacy to contribute to, take responsibility for, and shape the meanings that matter within a social configuration" (Wenger, 1998, p. 197, cited in Nuttall, 2004, p.170). Although her focus is teachers not children, her discussion resonates with the description of the two constructs children explored in the process of establishing their identities within the community.

\subsubsection{Alignment of methodological features}

This thesis contributes to the growing expertise in research that foregrounds children's voices. Like several other New Zealand qualitative theses, the emphasis was on children's perspectives (Brennan, 2005; Greenfield, 2007), but even more particularly on what children were doing together (Alcock, 2005). Both Brennan (2005) and I, for similar reasons, took the still unusual decision of not interviewing teachers or including teachers' voices, as a way of foregrounding the voices of children. Collectively these theses are building up clearer pictures of children's experience in early childhood education settings, and allowing some children's voices to be heard. A residual uneasiness is that other researchers may be selecting, as I overtly did, centres that are identified as 'high quality' for their research, and that voices of children in less favourable settings may still remain silent.

This thesis also contributes to the growing research expertise in participant observation (Alcock, 2005; Brennan, 2005; Hedges, 2007; Stephenson, 1998), but more particularly in the use of participatory approaches with young children (Brennan, 2002; Carr, 1997b; Greenfield, 2007; Hedges, 2007; Te One, 2005). Other New Zealand work, beyond this group of theses, is also relevant here (e.g., Carr, 2000; Smith et al., 2005). The work in this thesis contributes to a level of expertise in using participatory research strategies that is of international significance. In turn, such work builds on earlier New Zealand work (Hedges, 2002a; Cullen et al., 2005) in which the responsibilities and issues inherent in undertaking research with young children were spelt out. 


\subsection{Implications for the early childhood education profession}

In this section potential implications for the profession arising from the findings are described. In writing this, I have considered all levels of the professionteachers, teacher educators, researchers and policy makers-in offering avenues forward, in an effort to avoid Gore's (1992) critique of critical pedagogy as engendering its own regime of truth, which may be inaccessible to all but academics.

\subsubsection{The complexity of curriculum}

In Chapter 1 a need for research that focuses on curriculum was identified, particularly in light of growing interest in indirect aspects of curriculum (Alcock, 2005; Brennan, 2002, 2005, 2007; Carr, 1997a, 1997b, 2000; Hedges, 2007). Although the focus in this thesis has been on the boundaries of curriculum, it has highlighted the complexity of curriculum per se. Observations suggested the curriculum children were experiencing was far wider and more complex than is perhaps often acknowledged; it seems likely that our adult-centric focus leads us to overemphasise the significance of teachers as generators of curriculum, and to underestimate children's role in the process. The focus on curriculum boundaries and the role that the adult-child demarcation played in how these were defined highlighted the notion of curriculum as contested (Haggerty, 1998, 2003). The boundaries were indeed a site where "dominant and subordinate voices define and constrain each other, in battle and exchange" (Giroux, 1997, p.133), a setting in which generations struggle "to define themselves and the world" (Pinar et al., 1995, p.848). A further contributing factor to the complexity was the variation in individual children's experiences of curriculum. Again, it is likely that a focus on the collective group of children may mask how significantly individual children's experiences differ. These insights underscore the importance of curriculum as an area for continuing professional attention. But this attention needs to be directed to the messiness and complexity of curriculum as children experience it, to curriculum as "about life itself" (Hill, 2005, p.26), rather than to the neat packages of teacher intentions.

\subsection{Addressing generational issues}

Teachers had warm, loving relationships with children. Yet I have argued these were underpinned by wider social assumptions which positioned children as lesser and 'other' than adults. Mayall (2000) writes: 
Adults have divided up the social order into two major groups-adults and children, with specific conditions surrounding the lives of each group: provisions, constraints and requirements, laws, rights, responsibilities and privileges. Thus, just as the concept of gender has been key to understanding women's relationships to the social order, so the concept of generation is key to understanding childhood. (Mayall, 2000, pp.120-121)

I have argued that the influence of such generational thinking can and does operate within early childhood education to structure the ways we think, and the possibilities we can envisage. The discovery of how deeply the assumptions about adults and children were entrenched within the structure and organisation of the centre, and acted to embed the power imbalance between teachers and children, was an unanticipated and thought-provoking finding. McLaren (1998) identifies a structural contradiction facing schools: "the school functions simultaneously as a means of empowering students around issues of social justice and as a means of sustaining, legitimizing, and reproducing dominant class interests directed at creating obedient, docile, and low-paid future workers" (McLaren, 1998, p.172, italics in original). Here, analysis suggests there is an inherent contradiction between the professed focus on empowering children, and an embedded structure which acts to reinforce generational boundaries and so disempower them. While acknowledging the tension that exists "between agency and dependency" (Smith \& Taylor, 2000, p.4), when working with children who perhaps cannot yet walk or talk, acknowledging the pervasiveness of the 'regime of truth' that positions children as other and lesser is a step towards the reconsideration of children's and teachers' potential roles. The following suggestions relate to ways in which that typically unquestioned teacher:child demarcation might be blurred.

This research reinforces the need to debate the question "What image of children is implied by this practice?" As alluded to above, there is increasing evidence (Gibbons, 2005; Hill, 2003; McLeod, 2002; Nuttall, 2004) that more attention needs to be paid to teachers' beliefs, to how teachers can identify the image of the child that is reflected in their practices, how they can confront and resolve contradictions between this implied image and their professed beliefs, and how they can identify misalignments in their collective beliefs as a team.

Identifying misalignment between the implied image of children and the professed philosophy has particular significance in light of continuing findings that the profession is struggling with implementing the sociocultural principles of $T e$ whāriki (Bernstone, 2007; Brennan, 2005; Cullen, 2003a; Jordan, 2003; Nuttall, 
2004; Hedges, 2007; Hill, 2001, 2005). It seems likely that the assumptions around the generational demarcation between adults and children may be impeding teachers' attempts to implement these principles and may counteract teachers' commitment to the image of the child as competent, thoughtful and skilled. While the arguments put forward in this thesis may contribute to awareness of the social forces at work, this needs to be a continuing area of attention for the profession.

A focus on issues of power and control, and how these are exercised by teachers (Boyce, 2001) may be an uncomfortable but valuable area of professional focus. In particular, there needs to be a deeper understanding of the ways in which teachers can empower children, but with recognition of the inherent complexities within this concept. The notion of empowerment itself implies adults as powerful, and children as less powerful (Gore, 1992; Nuttall, 2004), which is inherently at odds with advocated sociocultural approaches. Further research, building on the work of Jordan (2003) and Bernstone (2007), which explores teachers' effectiveness in implementing strategies that blur the adult:child demarcation, and diminish the associated power imbalance would further assist teachers and teacher educators in understanding what positioning children as competent and capable learners can look like in practice. As Bernstone (2007) and Jordan (2003) have shown, teachers may not appreciate how infrequently children are offered powersharing, rather than power-allowing opportunities. Brandtzæg (2006) argues we convince ourselves that the choices we offer children allow them to be powerful "even though that same child is sent to a day care institution every day" (p. 91). A continuing focus on how children can be given authentic opportunities to experience responsibility and control and be positioned alongside teachers as competent and capable would help to blur the teacher:child power imbalance.

The social isolation of early childhood settings from the wider community is one aspect of the adult:child demarcation. Malaguzzi offers us the image of a child as "rich in potential, strong, powerful, competent, and, most of all, connected to other adults and children" (1993, p.10). Current assumptions about the isolation of teachers and children from the wider world make it difficult for children to have this conncection with the world of adults. Children in this centre were fortunate because there were opportunities for them to visit the school, the building site, and explore the grounds. Do other children have the same 
opportunities? Wright's (2003) image of the home-based care setting extending into the wider community provides food for thought for those in group care settings. Recognising the structural boundaries that exist, and the social assumptions and legislated requirements that underpin them, indicates the importance of finding ways in which these boundaries can be adjusted to enable children and teachers to more easily move in the wider world outside the centre walls.

Are there ways in which more of the real world can be brought into early childhood settings? Bringing visitors into a centre is one way of connecting children and teachers with the wider world. Again, these children were lucky; teachers recognised children's interest in the outside world and responded, for example, by inviting the police to bring the police dogs to visit. Who else could be invited? Could a truck and a group of workmen call in (Ministry of Education, 2004b)? Are there ways within the centre of giving children a greater role in socalled adult tasks? A fortuitously timed visit to a Montessori centre allowed me to see a boy (admittedly aged 5) choosing to undertake the task of ironing and folding all the laundered table napkins, which led me to wonder about what other centre tasks children might do. Again, children in this centre were fortunate; they had opportunities to be involved in authentic jobs such as gardening, constructing the house-frame, baking. Do children in all centres have such opportunities? Can children prepare food, fold washing, stack the dishwasher, put up wall displays? As noted in Chapter 1, some examples already exist; there are descriptions of children making mosaic tiles, cooking jam, growing potatoes (Ministry of Education, 2004b), making videos (Watson, 2005) and developing technological expertise (Ramsey et al., 2006).

One further aspect of bringing the real world into the lives of children is reconsidering how so-called 'difficult' topics are handled. Should children be shielded from issues of death, disaster or sickness? Scholars (Brennan, 2005; New et al., 2005; Silin, 1995) suggest children are aware of, and concerned about, such authentic life issues. Should we share with children the larger problems facing the world, such as global warming? There are no simple answers to these questions, but the contention (McLaren, 1998; Silin, 1995) that teachers take the concerns and questions of students as their starting place provides a useful platform for discussion. What is a teacher's role in the social issues in which children are daily 
engaging around friendship, inclusion and exclusion, gendered roles, and the exercising control and power with peers. Gender identity continues to be an area that attracts significant interest in early childhood research (Blaise, 2005; Davies, 1989; Jordan, 2002; Kyratzis, 2004; MacNaughton, 2000; Martin, 1998; Paley, 1984) although less so recently in New Zealand (Norris, 2001). Given the disproportionate teacher-time and energy which the older group of boys absorbed, and reports of similar groups in other settings (Jordan, 2002; Keddie, 2003) indicates this as an area for both professional and research focus. Those who advocate a transformational approach to curriculum (e.g., MacNaughton, 2003b) argue teachers have a responsibility to intervene more proactively in children's inequitable practices. Observing how easy it was for children to be positioned as 'outsider' by their peers, seeing how firmly children were entrenching the stereotypes they had met in other areas of their lives within the social world of the centre, and recognising how difficult it could be for children who attempted to resist these, has led me personally to shift towards a more transformative approach to curriculum.

A further way of blurring the adult:child demarcation relates to increasing children's participation in making decisions that affect their centre lives. Teachers frequently consulted individual children about their preferences; it is only a further step to allow children collectively a more significant role in centre decisions that affect them. Offering children such a role would parallel changes occurring in Scandinavia where practices are shifting towards "a situation where children actually influence and participate in planning of the education process" (Broström, 2006, p.230). Those examples (Emilson, 2007; Hắy, 2005) may provide further ideas for how children can be given greater influence within curriculum, and suggest methodologies to use in research on this topic.

Giving children greater input into decisions leads into the arena of children's rights, which is an emerging debate for the New Zealand profession (Te One, 2004, 2005). Laevers (2005) suggests the consequences of respecting children's rights are only just becoming apparent. He believes that an attitude of respect for children "goes beyond being 'nice' to children. Every phase in history where rights were given to a certain group of people, this went along with a deeper struggle and a dramatic change in the view of the world" (p. 21). The distinction Mayall (2002) draws between actor and agent is helpful in suggesting the way 
forward. She describes actor as implying "children are of the social world: beings rather than becomings" (p.236), whereas the use of agent implies that "children have an influence; their views are taken seriously" (p.236) and that they make a difference. Currently it seems we in the profession may still be struggling in making the transition to seeing children as beings rather than becomings. Acknowledging and allowing children to be agents, and advocating for them to have this role requires taking a further step.

\subsubsection{Recognising the complexity of children's social lives}

The complexity of children's social lives within the centre has been a continuing feature of the findings. While teachers were not interviewed, it seems likely that the complexity of children's social lives may not always be obvious to adults. Although the complexities of teacher-child and peer social interactions described here were just a tiny fraction of those that occurred during the research period, nevertheless they illustrate the intricacies and challenges children faced in their relationships. James (2005) conveys a sense of this in describing children's identities as "precarious" (p.323). She suggests "the tensions between individuality and conformity, between equality and hierarchy create a delicate framework around which children's identities are strung" (p.323). Her words evoke such struggles as Robert T.'s attempt to engage with others, Aidy's efforts to align himself with James, and Evie's rejection of the gendered roles. Very quickly my generational assumptions that children's social lives would be simple because they were young were dispelled. In many ways being unpractised in social skills, in the process of building relationships with peers, and being nonconformists to social niceties, made their relationships more challenging. Understanding the complexities children face highlights children's relationships and the processes of children establishing their identities within the centre community as important areas for professional attention. The complexities of peer relationships described here provide support for McCadden's (1998) assertion that teachers need to engage proactively with children who are having difficulties establishing themselves in the centre milieu. Teachers typically work proactively with children who are involved in conflict, but do other children whose behaviour attracts less immediate attention need their support just as keenly? Like Alcock (2005), I believe there is need for more research that focuses on children's peer relationships, and on how centres function as communities. 
The importance of particular parts of the centre day were highlighted by this thesis. Teacher-led communal group times, such as mat-times, are very significant opportunities when teachers can ensure all children know each other, but also can build a shared centre culture which will contribute to children's sense of collective identity and to their feelings of being a part of that community. The informality of mealtimes needs to be acknowledged and valued as a prime setting for children's interactions; it is a time when their sense of themselves as a group of peers with their own interests and culture can evolve. Mealtimes offer teachers an opportunity to engage with children and follow their agenda (Alcock, 2005).

\subsubsection{Adopting an attitude of openness}

Finally, and most fundamentally, this thesis demonstrates the importance of continuing to encourage openness, questioning and debate at all levels. It is important, Laevers (2005) argues, for educational models to adopt an 'open framework' approach, and for teachers to constantly ask themselves "how the organisation of the day, the materials and activities on offer, stimulating impulses and sensitive responses, can raise the quality of life of the child and support its development" (p.22). I would argue that the profession as a whole needs to adopt the same questioning approach. Although it is possible to consider that the issues raised here around the teacher:child demarcation, and the influence these have on curriculum, are problems imposed on early childhood education by wider social assumptions, there are many ways in which we, as a profession, unwittingly reinforce those assumptions. Adopting an open and questioning approach which encourages debate and critique is a way to guard against the profession becoming a closed community in which "old and tired knowledge" (Edwards, 2000, p.188) is recycled. A growing confidence to critique and challenge has been seen to be a characteristic of recent New Zealand research; it is important that these attitudes are encouraged at every level of the profession.

\subsection{Final words}

In these closing paragraphs, three things remain to be said: these concern the teachers, children, and the final purpose of this thesis. The overarching intent of this thesis has always been forward-looking, with an eye to future potential rather than present critique. However, it has done this by exploring the experiences of children and teachers in a single centre in ways that may appear at times critical of that setting and those teachers. I want again, at the end, to acknowledge the 
enormous contribution of the teachers whose dedication and passion for the profession and for their work with young children led them to agree to participate in this research. I am sensitive to the fact that others may take what I have intended as critique of the profession, and dismiss it as criticism of the team. The following words from Waksler (1991) are a timely reminder of what the close scrutiny of research might reveal for any of us:

In what follows, readers might be tempted to criticize the teacher's activities, policies, etc. I want to emphasize that the kind of close observation in which I engaged necessarily brings to light those features of everyday activity which are commonly concealed, ignored, covered up, explained away, etc. If every piece of my everyday life were brought forth for public scrutiny, behaviors would be disclosed that I myself might well deny or want to deny. (Waksler, 1991, p.100)

This thesis has argued for a reconceptualisation of the role which we as adults, allow for children within early childhood education. In doing this, it is reflecting a deeper social change that is taking place. At the beginning of the $21^{\text {st }}$ century, Prout (2005) suggests there is a feeling of the social order "fragmenting under the pressure of rapid economic, social and technological change" (p.7) and of a blurring in "the distinction between adults and children" (p.7). He believes these changes require new approaches that move beyond basic ideas such as 'children are actors in their own right' and urges for recognition that childhood is "like all phenomena, ... heterogeneous, complex and emergent" (Prout, 2005, p.2) and that understanding childhood will require "a broad set of intellectual resources, an interdisciplinary approach and an open-minded process of enquiry" (p.2). In a very small way this thesis may be seen both as a reflection of, and a contributor to, the process of blurring the distinction between adults and children, and as a small step towards the kind of open-minded process of enquiry that Prout proposes.

Finally, in the process of reviewing findings, aligning these with findings from other recent New Zealand theses, and identifying areas for future focus, all but one of the aims for this thesis have been revisited. The one remaining purpose was for the thesis to be 'a strategy of dislocation for others in assisting them to reexamine assumptions about early childhood education'. How well that purpose is achieved remains to be answered. 


\section{REFERENCES}

Ailwood, J. (2003). Governing early childhood education through play. Contemporary Issues in Early Childhood, 4(3), 286-299.

Aird, E. G. (2004). Advertising and marketing to children in the United States. In P. B. Pufall \& R. P. Unsworth (Eds.), Rethinking childhood (pp.141-153). New Brunswick: Rutgers University Press.

Alcock, S. (2005). A socio-cultural interpretation of young children's playful and humorous communication. Unpublished doctorate thesis, Massey University, Palmerston North.

Alcock, S. (2007). Playing with rules around routines: Children making mealtimes meaningful and enjoyable. Early Years, 27(3), 281-293.

Alldred, P. (1998). Ethnography and discourse analysis: Dilemmas in representing the voices of children. In J. Ribbens \& R. Edwards (Eds.), Feminist dilemmas in quantitative research: Public knowledge and private lives (pp.147-170). London: Sage.

Alloway, N. (1997). Early childhood education encounters the postmodern: What do we know? What can we count as 'true'? Australian Journal of Early Childhood, 22(2), 1-5.

Anderson, A. (2000). Making art with children - much more than 'doing it properly': A response to Felicity McArdle. Contemporary Issues in Early Childhood, 1(3), 329-332.

Anning, A. (1991). The first years of school. Milton Keynes: Oxford University Press.

Anning, A. (2004). The co-construction of an early childhood curriculum. In A. Anning, J. Cullen \& M. Fleer (Eds.), Early childhood education: Society and culture (pp.57-68). London: Sage.

Anning, A., \& Edwards, A. (2006). Promoting children's learning from birth to five: Developing the new early years professional ( $\left.2^{\text {nd }} \mathrm{ed}.\right)$. Maidenhead: Open University Press.

Apple, M. W. (2004). Ideology and curriculum ( $3^{\text {rd }}$ ed.). New York: RoutledgeFalmer.

Apple, M. W., \& King, N. R. (1977). What do schools teach? Curriculum Inquiry, 6(4), 341-358.

Arthur, L. (2001). Popular culture and early literacy learning. Contemporary Issues in Early Childhood, 2(3), 295-307. 
Ashton, J. (2005). Barbie, the Wiggles and Harry Potter. Can popular culture really support young children's literacy development. European Early Childhood Education Research Journal, 13(1), 31-40.

Athey, C. (1990). Extending thought in young children: A parent-teacher partnership. London: Chapman.

Barker, P. (1991). Michel Foucault: An introduction. Edinburgh: Edinburgh University Press.

Bassey, M. (2003). Case study research. In J. Swann \& J. Pratt (Eds.), Educational research in practice: Making sense of methodology. London: Continuum.

Batycky, J. (2008). Early childhood voices: Who is really talking? Contemporary Issues in Early Childhood, 9(2), 173-177.

Bayes, C. (1999). Making Reggio our own. Wellington: Winston Churchill Memorial Trust.

Berg, B. L. (2004). Qualitative research methods for social sciences $\left(5^{\text {th }}\right.$ ed.). Boston: Pearson Education.

Bergen, D., Reid, R., \& Torelli, L. (2001). Educating and caring for very young children: The infant/toddler curriculum. New York: Teachers College Press.

Bernstein, B. (1997). Class and pedagogies - Visible and invisible. In A. H. Halsey, H. Lauder, P. Brown \& A. S. Wells (Eds.), Education: Culture, economy and society (pp.59-79). New York: Oxford University Press. (Original work published 1975)

Bernstein, B. (2000). Pedagogy, symbolic control and identity (Rev. ed.). Lanham, MD: Rowman \& Littlefield.

Bernstone, H. (2007). The relationship between the beliefs of early childhood teachers and their use of scaffold, instruction and negotiation as teaching strategies. Unpublished doctorate thesis, Brunel University, London.

Birbeck, D., \& Drummond, M. (2005). Interviewing, and listening to the voices of very young children on body image and perceptions of self. Early Child Development and Care, 175(6), 579-596.

Blaise, M. (2005). Performing femininities through gender discourses. In L. D. Soto \& B. B. Swadener (Eds.), Power and voice in research with children (pp.105-116). New York: Peter Lang.

Blaise, M., \& Andrew, Y. (2005). How 'bad' can it be? Troubling gender, sexuality, and early childhood teaching. In N. Yelland (Ed.), Critical issues in early childhood education (pp.49-57). Maidenhead: Open University. 
Bloch, M. N. (1992). Critical perspectives on the historical relationship between child development and early childhood education research. In S. A. Kessler \& B. B. Swadener (Eds.), Reconceptualizing the early childhood curriculum (pp.3-20). New York: Teachers College Press.

Bloch, M. N., \& Popkewitz, T. S. (2000). Constructing the parent, teacher, and child: Discourses of development. In L. D. Soto (Ed.), The politics of early childhood education (pp.7-32). New York: Peter Lang.

Bodrova, E., \& Leong, D. J. (2003). The importance of being playful. Educational Leadership, 60(7), 50-53.

Bolstad, R. (2004). The role and potential of ICT in early childhood education: A review of New Zealand and international literature. Wellington: New Zealand Council for Educational Research.

Bone, J. (2005). An ethical journey: Rights, relationships and reflexivity. Australian Journal of Early Childhood, 30(1), 1-5.

Bone, J., Cullen, J., \& Loveridge, J. (2007). Everyday spirituality: An aspect of the holistic curriculum in action. Contemporary Issues in Early Childhood, 8(4), 344-354.

Bowen, G. A. (2006). Grounded theory and sensitizing concepts. International Journal of Qualitative Methods, 5(3), 1-9.

Boyce, D. (2001). Issues of power in early childhood education: Reflections of a beginning teacher. The First Years: Ngā Tau Tuatahi New Zealand Journal of Infant and Toddler Education, 3(2), 6-9.

Brandtzæg, K.-M. (2006). Underground: Early childhood and ageism in a perspective of power and disempowerment. Journal of Australian Research in Early Childhood Education, 13(2), 91-101.

Brennan, M. (1999). Playing in the zone: A Vygotskian analysis of televisioninspired play. Unpublished master's thesis, Massey University, Palmerston North.

Brennan, M. (2001). Television advertising: Children participating in adult worlds. Early Education, (26), 23-30.

Brennan, M. (2002). "She can't be Baby Spice... Because she's got black hair!" Young children talking television. New Zealand Research in Early Childhood Education, 5, 133-143.

Brennan, M. (2005). "They just want to be with us". Young children learning to live the culture: A post-Vygotskian analysis of young children's 
enculturation into a childcare setting. Unpublished doctorate thesis, Victoria University, Wellington.

Brennan, M. (2007). Young children breaking the rules: A sociocultural interpretation. Early Childhood Folio, 11, 2-4.

Broström, S. (2003). Understanding Te Whäriki from a Danish perspective. In J. Nuttall (Ed.), Weaving Te Whāriki: Aotearoa New Zealand's early childhood curriculum document in theory and practice (pp.215-242). Wellington: New Zealand Council for Educational Research.

Broström, S. (2006). Children's perspectives on their childhood experiences. In J. Einarsdottir \& J. T. Wagner (Eds.), Nordic childhoods and early education: Philosophy, research, policy, and practice in Denmark, Finland, Iceland, Norway, and Sweden (pp.223-255). Greenwich, CT: Information Age Publishing.

Browne, N. (2004). Gender equity in the early years. Maidenhead: Open University Press.

Brownlee, P. (2000). Computers and playcentre: Are they compatible. The First Years: Ngā Tau Tuatahi New Zealand Journal of Infant and Toddler Education, 2(1), 21-22.

Brownlee, P. (2004). Magic places: A guide for adults to encourage young children's creative art work (Rev. ed.). Auckland: New Zealand Playcentre Federation.

Bryant, A., \& Charmaz, K. (Eds.). (2007). The Sage handbook of grounded theory. Los Angeles: Sage.

Burbules, N. C., \& Berk, R. (1999). Critical thinking and critical pedagogy: Relations, differences, and limits. In T. S. Popkewitz \& L. Fendler (Eds.), Critical theories of education (pp.45-65). New York: Routledge.

Burton, D. (2000). The use of case studies in social science research. In D. Burton (Ed.), Research training for social scientists. London: Sage.

Campbell, S. (1999). Making the political pedagogical in early childhood. Australian Journal of Early Childhood, 24(4), 21-26.

Campbell, S., \& Smith, K. (2001). Equity observation and images of fairness in childhood. In S. Grieshaber \& G. S. Cannella (Eds.), Embracing identities in early childhood education: Diversity and equity (pp.89-102). New York: Teachers College Press.

Cannella, G. S. (1997). Deconstructing early childhood education: Social justice and revolution. New York: Peter Lang. 
Cannella, G. S. (1999). The scientific discourse of education: Predetermining the lives of others - Foucault, education, and children. Contemporary Issues in Early Childhood, 1(1), 36-44.

Cannella, G. S., \& Viruru, R. (1997). Privileging child-centered play-based instruction. In G. S. Cannella, Deconstructing early childhood education: Social justice and revolution (pp.117-136). New York: Peter Lang.

Cannella, G. S., \& Viruru, R. (2002). (Euro-American constructions of) education of children (and adults) around the world: A postcolonial critique. In G. S. Cannella \& J. L. Kincheloe (Eds.), Kidworld: Childhood studies, global perspectives, and education (pp.197-213). New York: Peter Lang.

Cannella, G. S., \& Viruru, R. (2004). Childhood and postcolonization: Power, education, and contemporary practice. New York: RoutledgeFalmer.

Carlsson-Paige, N., \& Levin, D. E. (1990). Who's calling the shots?: How to respond effectively to children's fascination with war play and war toys. Philadelphia: New Society.

Carpenter, V. M. (2001). Curriculum and the (re)production of education. In V. M. Carpenter, H. Dixon, E. Rata \& C. Rawlinson (Eds.), Theory in practice for educators (pp.109-136). Palmerston North: Dunmore Press.

Carr, M. (1997a). Persistence when it's difficult: A disposition to learn for early childhood. Early Childhood Folio 3, 9-12.

Carr, M. (1997b). Technological practice in early childhood as a dispositional milieu. Unpublished doctorate thesis, University of Waikato, Hamilton.

Carr, M. (1998). Assessing children's learning in early childhood settings [kit]. Hamilton, Wellington: University of Waikato; New Zealand Council for Educational Research.

Carr, M. (2000). Seeking children's perspectives about their learning. In A. B. Smith, N. J. Taylor \& M. M. Gollop (Eds.), Children's voices: Research, policy and practice (pp.37-55). Auckland: Pearson Educational.

Carr, M. (2001). Assessment in early childhood settings: Learning stories.

London: Paul Chapman.

Carr, M., \& Claxton, G. (2004). Tracking the development of learning dispositions. In H. Daniels \& A. Edwards (Eds.), The RoutledgeFalmer reader in psychology of education (pp.106-131). London:

RoutledgeFalmer.

Carr, M., Hatherly, A., Lee, W., \& Ramsey, K. (2003). Te Whāriki and assessment: A case study of teacher change. In J. Nuttall (Ed.), Weaving 
Te Whäriki: Aotearoa New Zealand's early childhood curriculum document in theory and practice (pp.187-212). Wellington: New Zealand Council for Educational Research.

Carr, M., \& May, H. (1996). Te Whāriki, making a difference for the under-fives?:

The new national early childhood curriculum. Delta, 48(1), 101-112.

Carr, M., \& May, H. (2000). Te Whāriki: Curriculum voices. In H. Penn (Ed.),

Early childhood services: Theory, policy and practice (pp.53-73).

Buckingham: Open University Press.

Carr, M., May, H., Podmore, V. N., with, Cubey, P., Hatherly, A., et al. (1999).

Learning and teaching stories: Action research on evaluation in early childhood. Final report to the Ministry of Education. Wellington: New Zealand Council for Educational Research.

Carspecken, P. F. (1996). Critical ethnography in educational research: A theoretical and practical guide. New York: Routledge.

Carspecken, P. F., \& MacGillivray, L. (1998). Raising consciousness about reflection, validity, and meaning. In G. Shacklock \& J. Smyth (Eds.), Being reflexive in critical educational and social research (pp.171-189). London: Falmer.

Charmaz, K. (2003). Grounded theory: Objectivist and constructivist methods. In N. K. Denzin \& Y. S. Lincoln (Eds.), Strategies of qualitative inquiry (pp.249-291). Thousand Oaks, CA: Sage.

Charmaz, K. (2006). Constructing grounded theory: A practical guide through qualitative analysis. London: Sage.

Christensen, P., \& James, A. (Eds.). (2000). Research with children: Perspectives and practices. London: Falmer.

Clark, A. (2004). The mosaic approach and research with young children. In V. Lewis, M. Kellett, C. Robinson, S. Fraser \& S. Ding (Eds.), The reality of research with children and young people (pp.142-156). London: Sage.

Clark, A. (2007). A hundred ways of listening: Gathering children's perspectives of their early childhood environment. Young Children, 62(3), 76-81.

Clark, A., \& Moss, P. (2001). Listening to young children: The mosaic approach. London: National Children's Bureau and Joseph Rowntree Foundation.

Claxton, G. (1999). Wise up: The challenge of lifelong learning. London:

Bloomsbury. 
Claxton, G. (2002). Education for the learning age: A sociocultural approach to learning to learn. In G. Wells \& G. Claxton (Eds.), Learning for life in the 21 st century (pp.21-33). Oxford: Blackwell.

Cobb, C., Danby, S., \& Farrell, A. (2005). Governance of children's everyday spaces. Australian Journal of Early Childhood, 30(1), 14-20.

Connerton, B., \& Patterson, C. (2006). Growing into documenting: Stories of war and secret places. In A. Fleet, C. Patterson \& J. Robertson (Eds.), Insights: Behind early childhood pedagogical documentation (pp.97-113). Castle Hill: Pademelon.

Connolly, P. (1998). Racism, gender identities and young children: Social relations in a multi-ethnic, inner-city primary school. London: Routledge.

Corsaro, W. A. (1985). Friendship and peer culture in the early years. Norwood, $\mathrm{NJ}$ : Ablex.

Corsaro, W. A. (1997). The sociology of childhood. Thousand Oaks, CA: Pine Forge Press.

Corsaro, W. A. (2003). We're friends right?: Inside kids' culture. Washington, DC: Joseph Henry.

Corsaro, W. A., \& Nelson, E. (2003). Children's collective activities and peer culture in early literacy in American and Italian preschools. Sociology of Education, 76(3), 209-227.

Corsaro, W. A., \& Molinari, L. (2000). Entering and observing in children's worlds: A reflection on a longitudinal ethnography of early education in Italy. In P. Christensen \& A. James (Eds.), Research with children: Perspectives and practices (pp.179-200). London: Falmer.

Costa, A. L., \& Kallick, B. (2000). Preface to the series. In A. L. Costa \& B. Kallick (Eds.), Activating and engaging habits of mind (pp. xii-xvi). Alexandria, VA: Association for Supervision and Curriculum Development.

Cremin, H., \& Slatter, B. (2004). Is it possible to access the 'voice' of pre-school children? Results of a research project in a pre-school setting. Educational Studies, 30(4), 457-470.

Crook, S. (Ed.). (2004). Just improvise! Innovative play experiences for children under eight. Croydon, Australia: Tertiary Press.

Cuffaro, H. K. (1995). Experimenting with the world: John Dewey and the early childhood classroom. New York: Teachers College Press. 
Cullen, J. (1996). The challenge of Te Whäriki for future developments in early childhood education, Delta, 48(1), 113-126.

Cullen, J. (1999). Children's knowledge, teachers' knowledge: Implications for early childhood teacher education. Australian Journal of Teacher Education, 24(2), 15-25.

Cullen, J. (2003a). The challenge of Te Whāriki: Catalyst for change. In J. Nuttall (Ed.), Weaving Te Whāriki: Aotearoa New Zealand's early childhood curriculum document in theory and practice (pp.269-296). Wellington:

New Zealand Council for Educational Research.

Cullen, J. (2003b). The growth of an early childhood research culture: Implications for future directions in early childhood research. New Zealand Research in Early Childhood Education, 6, 1-12.

Cullen, J., Hedges, H., \& Bone, J. (2005). Planning, undertaking and disseminating research in early childhood settings: An ethical framework. ChildForum Early Childhood Research Network. Retrieved December 12, 2008, from http://www.childform.com/publication_details.asp?REF_NO=17

Curtis, A. (1998). A curriculum for the pre-school child: Learning to learn. London: Routledge.

Dahlberg, G., \& Moss, P. (2005). Ethics and politics in early childhood education. London: RoutledgeFalmer.

Dahlberg, G., Moss, P., \& Pence, A. (1999). Beyond quality in early childhood education and care: Postmodern perspectives. London: Falmer.

Dalli, C. (1999). Starting childcare before three: Narratives of experience from a tri-partite focus. Unpublished doctorate thesis, Victoria University, Wellington, New Zealand.

Dalli, C., \& Te One, S. (2002). Early childhood education in 2002: Pathways to the future. New Zealand Annual Review of Education, 12, 177-202.

Danby, S. (1997). "What's the problem?" One teacher's response to conflict in an early childhood classroom. In M. Goos, K. Moni \& J. Knight (Eds.), Scholars in context: Prospects and transitions (pp.23-27). Brisbane: Post Pressed.

Danby, S., \& Farrell, A. (2004). Accounting for young children's competence in educational research: New perspectives on research ethics. The Australian Educational Researcher, 31(3), 35-49. 
Darder, A., Baltodano, M., \& Torres, R. D. (2003). Critical pedagogy: An introduction. In A. Darder, M. Baltodano \& R. D. Torres (Eds.), The critical pedagogy reader (pp.1-21). New York: RoutledgeFalmer.

Davies, B. (1989). Frogs and snails and feminist tales: Preschool children and gender. Sydney: Allen \& Unwin.

Davies, B. (1993). Shards of glass: Children reading and writing beyond gendered identities. Sydney: Allen \& Unwin.

Davies, C. A. (1999). Reflexive ethnography: A guide to researching ourselves and others. London: Routledge.

Davis-Seaver, J. (2000). Critical thinking in young children. New York: Edwin Mellen Press.

Denscombe, (2007). The good research guide for small-scale social research projects $\left(3^{\text {rd }}\right.$ ed.). Maidenhead: Open University.

Denzin, N. K. (1997). Interpretive ethnography: Ethnographic practices for the 21st century. Thousand Oaks, CA: Sage.

Denzin, N. K., \& Lincoln, Y. S. (2002). The qualitative inquiry reader. Thousand Oaks, CA: Sage.

Dockett, S., \& Fleer, M. (1999). Play and pedagogy in early childhood: Bending the rules. Sydney: Harcourt.

Dockrell, J., Lewis, A., \& Lindsay, G. (2000). Researching children's perspectives: A psychological dimension. In A. Lewis \& G. Lindsay (Eds.), Researching children's perspectives (pp.46-58). Buckingham: Open University Press.

Drummond, M.-J. (1999). Another way of seeing: Perceptions of play in a Steiner kindergarten. In L. Abbott \& H. Moylett (Eds.), Early education transformed (pp.48-60). United Kingdom: Falmer.

Drummond, M.-J. (2000). Starting with children: Towards an early years curriculum. In C. Clouder, S. Jenkinson \& M. Large (Eds.), The future of the child (pp.27-36). Stroud, UK: Hawthorn Press.

Duncan, J. (1998). I spy: Sexual abuse prevention policies - protection or harm? Wellington: Institute for Early Childhood Studies, Victoria University.

Duncan, J. (1999). New Zealand kindergarten teachers and sexual abuse protection policies. Teaching and Teacher Education, 15, 243-252.

Duncan, J. (2004, May). "She's always been, what I would think, a perfect daycare child": Constructing the subjectivities of a New Zealand child. Paper 
presented at the 12th Reconceptualizing Early Childhood Education Conference on Research, Theory and Practice, Oslo.

Duncan, J., Jones, C., \& Carr, M. (2008). Learning dispositions and the role of mutual engagement: Factors for consideration in educational settings. Contemporary Issues in Early Childhood, 9(2), 107-117.

Edwards, A. (2000). Research and practice: Is there a dialogue? In H. Penn (Ed.), Early childhood services (pp.184-199). Buckingham: Open University Press.

Edwards, C., Gandini, L., \& Forman, G. (Eds.). (1994). The hundred languages of children: The Reggio Emilia approach to early childhood education. Norwood, NJ: Ablex.

Einarsdottir, J. (2005). Playschool in pictures: Children's photographs as a research method. Early Child Development and Care, 175(6), 523-541.

Einarsdottir, J. (2006). Between two continents, between two traditions:

Education and care in Icelandic preschools. In J. Einarsdottir \& J. T. Wagner (Eds.), Nordic childhoods and early education: Philosophy, research, policy and practice in Denmark, Finland, Iceland, Norway, and Sweden (pp.159-182). Greenwich, CT: Information Age.

Einarsdottir, J. (2007). Research with children: Methodological and ethical challenges. European Early Childhood Education Research Journal, 15(2), 197-211.

Eisner, E. W. (1985). The educational imagination: On the design and evaluation of school programs (2 $2^{\text {nd }}$ ed.). New York: Macmillan.

Ellsworth, E. (1989). Why doesn't this feel empowering?: Working through the repressive myths of critical pedagogy. Harvard Educational Review, 59(3), 297-324.

Emilson, A. (2007). Young children's influence in preschool. International Journal of Early Childhood, 39(1), 11-38.

Engeström, Y. (1987). Learning by expanding: An activity-theoretical approach to developmental research. Helsinki: Orienta-Konsultit.

Engeström, Y. (1999). Activity theory and individual and social participation. In Y. Engeström, R. Miettinen \& R.-L. Punamaki (Eds.), Perspectives on activity theory (pp.19-38). Cambridge: Cambridge University Press.

Epstein, A. S. (2003). How planning and reflection develop young children's thinking skills. Young Children, 58(5), 28-36. 
Fabes, R. A., Martin, C. L., \& Hanish, L. D. (2004). The next 50 years: Considering gender as a context for understanding young children's peer relationships. Merrill-Palmer Quarterly, 50(3), 260-274.

Farquhar, S., \& Fleer, M. (2007). Developmental colonisation of early childhood education in Aotearoa/New Zealand and Australia. In L. Keesing-Styles \& H. Hedges (Eds.), Theorising early childhood practices: Emerging dialogues (pp.27-49). Castle Hill: Pademelon.

Farquhar, S.-E. (2003). Quality teaching: Early foundations best evidence synthesis. Wellington: Ministry of Education.

Farrant, A., \& Wright, J. (2007). Relationships in action in the area of visual art: Alana's story of learning at New Beginnings Pre-school. In A. Meade (Ed.), Cresting the waves: Innovation in early childhood education (pp.1928). Wellington: New Zealand Council for Educational Research.

Farrell, A., Tayler, C., \& Tennent, L. (2002). Early childhood services: What can children tell us? Australian Journal of Early Childhood, 27(3), 13-17.

Fasoli, L. (2003). Reflections on doing research with young children. Australian Journal of Early Childhood, 28(1), 7-11.

Fleer, M. (1998). 'Me not a boy, me a person!': Deconstructing gendered interactional patterns in early childhood. Australian Journal of Early Childhood, 23(1), 22-28.

Fleer, M. (2003a). Early childhood education as an evolving 'community of practice' or as lived 'social reproduction': Researching the 'taken-forgranted'. Contemporary Issues in Early Childhood, 4(1), 64-79.

Fleer, M. (2003b). The many voices of Te Whāriki: Kaupapa Māori, sociocultural, developmental, constructivist and ...? Australians listen carefully. In J. Nuttall (Ed.), Weaving Te Whāriki: Aotearoa New Zealand's early childhood curriculum document in theory and practice (pp.243-268). Wellington: New Zealand Council for Educational Research.

Fleer, M. (2006). Troubling cultural fault lines: Some indigenous Australian families' perspectives on the landscape of early childhood education. Mind, Culture and Activity, 13(3), 191-204.

Fleer, M., \& Robbins, J. (2004). "Yeah that's what they teach you at uni, It's just rubbish": The participatory appropriation of new cultural tools as early childhood student teachers move from a developmental to a sociocultural framework for observing and planning. Journal of Australian Research in Early Childhood Education, 11(1), 47-62. 
Fleer, M., \& Robbins, J. (2007). A cultural-historical analysis of early childhood education: How do teachers appropriate new cultural tools? European Early Childhood Education Research Journal, 15(1), 103-119.

Fleet, A. (2002). Revisiting adult work in early childhood settings: Shifting the frame. Australian Journal of Early Childhood, 27(1), 18-23.

Flewitt, R. (2005). Conducting research with young children: Some ethical considerations. Early Child Development and Care, 175(6), 553-565.

Flinders, D. J., Noddings, N., \& Thornton, S. J. (1986). The null curriculum: Its theoretical basis and practical implications. Curriculum Inquiry, 16(1), 3342.

Formosinho, J., \& Araujo, S. B. (2004). Children's perspectives about pedagogical interactions. European Early Childhood Education Research Journal, 12(1), 103-114.

Formosinho, J., \& Araujo, S. B. (2006). Listening to children as a way to reconstruct knowledge about children: Some methodological implications. European Early Childhood Education Research Journal, 14(1), 21-31.

Fraser, S., \& Gestwicki, C. (2000). Authentic childhood: Exploring Reggio Emilia in the classroom. New York: Delmar.

Freire, P. (1990). Pedagogy of the oppressed. London: Penguin. (Original work published 1972)

Freire, P., \& Macedo, D. P. (1987). Literacy: Reading the word and the world. South Hadley, MA: Bergin \& Garvey.

Gair, M., \& Mullins, G. (2001). Hiding in plain sight. In E. E. Margolis (Ed.), The hidden curriculum in higher education (pp.21-41). New York: Routledge.

Gandini, L. (2005). From the beginning of the atelier to materials as languages. In L. Gandini, L. Hill, L. Cadwell \& C. Schwall (Eds.), In the spirit of the studio: Learning from the atelier of Reggio Emilia (pp.6-15). Columbia: Teachers College Press.

Gandini, L., Hill, L., Cadwell, L., \& Schwall, C. (Eds.). (2005). In the spirit of the studio: Learning from the atelier of Reggio Emilia. New York: Teachers College Press.

Garbett, D. (2004). Science education in early childhood teacher education: Putting forward a case to enhance student teachers' confidence and competence. Research in Science Education, 33(4), 467-481.

Gibbons, A. (2007). Playing the ruins: The philosophy of care in early childhood education. Contemporary Issues in Early Childhood, 8(2), 123-131. 
Gibbons, P. (2004). Learning through participation in a playcentre routine. Unpublished master's thesis, Massey University, Palmerston North.

Gibbons, P. (2005). "The way we do things around here": Environmental and social considerations of the organisational culture of two playcentres. New Zealand Research in Early Childhood Education, 8, 11-27.

Giroux, H. A. (1997). Pedagogy and the politics of hope: Theory, culture, and schooling. Boulder, CO: Westview Press.

Giroux, H. A. (2001). Theory and resistance in education: Towards a pedagogy for the opposition (Rev.ed.). Westport, CT: Bergin \& Garvey.

Giugni, M. (2006). The power and the passion: Popular culture and pedagogy. In A. Fleet, C. Patterson \& J. Robertson (Eds.), Insights: Behind early childhood pedagogical documentation (pp.205-224). Castle Hill: Pademelon.

Goffin, S. G., \& Wilson, C. S. (2001). Curriculum models and early childhood education: Appraising the relationship ( $2^{\text {nd }}$ ed.). Upper Saddle River, NJ: Prentice Hall.

Gonzalez-Mena, J. (1997). Multicultural issues in child care $\left(2^{\text {nd }}\right.$ ed.). Mountain View, CA: Mayfield.

Gore, J. (1992). What we can do for you! What can "we" do for "you"?: Struggling over empowerment in critical and feminist pedagogy. In C. Luke \& J. Gore (Eds.), Feminisms and critical pedagogy (pp.54-73). New York: Routledge.

Gore, J. (1993). The struggle for pedagogies: Critical and feminist discourses as regimes of truth. New York: Routledge.

Goulart, M. I. M., \& Roth, W.-M. (2006). Margin|centre: Towards a dialectic view of participation. Journal of Curriculum Studies, 38(6), 679-700.

Graue, M. E. (2005). (De) centering the kindergarten prototype in the childcentered classroom. In S. Ryan \& S. Grieshaber (Eds.), Practical transformations and transformational practices: Globalization, postmodernism, and early childhood education (pp.39-58). Amsterdam: Elsevier.

Graue, M. E., \& Walsh, D. J. (1998). Studying children in context: Theories, methods, and ethics. Thousand Oaks, CA: Sage.

Grebennikov, L. (2006). Preschool teachers' exposure to classroom noise. International Journal of Early Years Education, 14(1), 35-44. 
Greenfield, C. (2004). Transcript: 'Can run, play on bikes, jump on the zoom slide, and play on the swings': Exploring the value of outdoor play. Australian Journal of Early Childhood, 29(2), 1-5.

Greenfield, C. (2007). A case study of children's and adults' perceptions of "being outside" in one early childhood centre. Unpublished master's thesis, Massey University, Palmerston North.

Greenman, J. (1998). Places for childhoods: Making quality happen in the real world. Redmond, WA: Child Care Information Exchange.

Greenman, J. (2005). Caring spaces, learning places: Children's environments that work. Redmond, WA: Exchange.

Greenman, J. (2007). Places to live: Important dimensions of child care settings. Exchange, (178), 21-24.

Grey, A. (1974). Learning through play. Auckland: New Zealand Playcentre Federation.

Grieshaber, S. (2001). Beating Mom: How to win the power game. In J. A. Jipson \& R. T. Johnson (Eds.), Resistance and representation: Rethinking childhood education (pp.223-237). New York: Peter Lang.

Gross, T., \& Clemens, S. G. (2002). Painting a tragedy: Young children process the events of September 11. Young Children, 57(3), 44-51.

Gruss, C., Jackson, I., Grimson, A., \& Hedgcock, D. (1998). Antisocial play in the preschool: Survey data on children's behaviour and some response strategies in current use. Australian Journal of Early Childhood, 23(1), 1015.

Gunn, A. (2006). Rainbow families: A selection of children's literature inclusive of 'rainbow families' and 'queer' cultures. Early Education, (39), 29-31.

Haas, M. (1996). Children in the junkyard. Childhood Education, 72(6), 345-351.

Hadley, K. G., \& Nenga, S. K. (2004). From Snow White to Digimon. Childhood, 11(4), 515-536.

Haggerty, M. (1998). Sighting, citing and siting Te Whäriki: Exploring the use of video feedback as a tool for critical pedagogy. The experience of five early childhood centres. Unpublished master's thesis, Victoria University, Wellington.

Haggerty, M. (2003). Reconceptualising notions of curriculum: The case of $T e$ Whāriki. New Zealand Research in Early Childhood Education, 6, 35-49.

Hammersley, M., \& Atkinson, P. (1995). Ethnography: Principles in practice $\left(^{\text {nd }}\right.$ ed.). London: Routledge. 
Håøy, A. (2005). Children's say in early childhood education. Australian Journal of Early Childhood, 12(1), 59-67.

Harper, S. (2004). Schemas in areas of play. Playcentre Journal, (121), 8-9.

Hedges, H. (2002a). Beliefs and principles in practice: Ethical research with child participants. New Zealand Research in Early Childhood Education, 5, 3147.

Hedges, H. (2002b). Subject content knowledge in early childhood curriculum and pedagogy. Unpublished master's thesis, Massey University, Palmerston North.

Hedges, H. (2003). Teaching and learning: Theories that underpin 'wise' practice in Aotearoa/New Zealand. Early Education, (31), 5-12.

Hedges, H. (2007). Funds of knowledge in early childhood communities of inquiry. Unpublished doctorate thesis, Massey University, Palmerston North.

Hedges, H., \& Cullen, J. (2005). Meaningful teaching and learning: Children's and teachers' content knowledge. ACE papers, (16), 11-24.

Hill, D. (2001). Passion, power and planning in the early childhood centre. The First Years: Ngā Tau Tuatahi New Zealand Journal of Infant and Toddler Education, 3(2), 10-13.

Hill, D. (2003). Theory as story: A framework for understanding and articulating the narrative of personal practice. The First Years: Ngā Tau Tuatahi New Zealand Journal of Infant and Toddler Education, 5(2), 24-26.

Hill, D. (2005). Curriculum: Challenges of context and complexity in early childhood settings. The First Years: Ngā Tau Tuatahi New Zealand Journal of Infant and Toddler Education, 7(1), 21-26.

Holzwarth, W., \& Erlbruch, W. (1993). The story of the little mole who went in search of whodunit. Pymble, NSW: HarperCollins.

Hood, J. C. (2007). Orthodoxy vs. power: The defining traits of grounded theory. In A. Bryant \& K. Charmaz (Eds.), The Sage handbook of grounded theory (pp. 151-164). Los Angeles: Sage.

hooks, b. (1994). Eros, eroticism, and the pedagogical process. In H. A. Giroux \& P. McLaren (Eds.), Between borders: Pedagogy and politics of cultural studies (pp.113-118). London: Routledge.

Howard, J. (2002). Eliciting children's perceptions. Early Child Development and Care, 172(5), 489-502. 
Hughes, F. P. (2003). Spontaneous play in the 21 st century. In O. N. Saracho \& B. Spodek (Eds.), Contemporary perspectives on play in early childhood education (pp.21-39). Greenwich, CT: IAP.

Hughes, P., \& MacNaughton, G. (2001). Fractured or manufactured: Gendered identities and culture in the early years. In S. Grieshaber \& G. S. Cannella (Eds.), Embracing identities in early childhood education: Diversity and possibilities (pp.114-130). New York: Teachers College Press.

Hyun, E., \& Dong, H. C. (2004). Examination of young children's gender-doing and gender-bending in their play dynamics: A cross-cultural exploration. International Journal of Early Childhood, 36(1), 49-65.

Isaacs, S. (1933). Social development in young children. London: Routledge \& Kegan Paul.

Jackson, P. W. (1968). Life in classrooms. New York: Holt, Rinehart \& Winston. James, A. (2004). Understanding childhood from an interdisciplinary perspective. In P. B. Pufall \& R. P. Unsworth (Eds.), Rethinking childhood (pp.25-37). New Brunswick: Rutgers University Press.

James, A. (2005). Learning to be friends: Methodological lessons from participant observation among English schoolchildren. Childhood, 3(3), 313-329.

James, A., Jenks, C., \& Prout, A. (1998). Theorizing childhood. New York: Teachers College Press.

James, A., \& Prout, A. (1990). Constructing and reconstructing childhood: Contemporary issues in the sociological study of childhood. London: Falmer.

Janmohamed, A. R. (1994). Some implications of Paulo's Freire's border pedagogy. In H. A. Giroux \& P. McLaren (Eds.), Between borders: Pedagogy and the politics of cultural studies (pp.242-252). London: Routledge.

Jarvis, P. (2007). Monsters, magic and Mr Psycho: A biocultural approach to rough and tumble play in the early years of primary school. Early Years, 27(2), 171-188.

Jenks, C. (1996). Childhood. London: Routledge.

Jenks, C. (2000). Zeitgeist research on childhood. In P. Christensen \& A. James (Eds.), Research with children: Perspectives and practices (pp.62-76). London: Falmer. 
Jipson, J. A. (2000). The stealing of wonderful ideas: The politics of imposition and representation in research on early childhood. In L. D. Soto (Ed.), The politics of early childhood education (pp.167-177). New York: Peter Lang.

Jipson, J. A. (2001). Introduction: Resistance and representation: Rethinking childhood education. In J. A. Jipson \& R. T. Johnson (Eds.), Resistance and representation: Rethinking childhood education (pp.1-12). New York: Peter Lang.

Jipson, J. A., \& Johnson, R. T. (Eds.). (2001). Resistance and representation: Rethinking childhood education. New York: Peter Lang.

Jones, E., \& Nimmo, J. (1994). Emergent curriculum. Washington DC: National Association for the Education of Young Children.

Jones, L. (2002). Derrida goes to nursery school: Deconstructing young children's stories. Contemporary Issues in Early Childhood, 3(1), 139-146.

Jones, N. P. (2005). Big jobs: Planning for competence. Young Children, 60(2), 86-93.

Jordan, B. (2003). Professional development making a difference for children: Co-constructing understandings in early childhood centres. Unpublished doctorate thesis, Massey University, Palmerston North.

Jordan, B. (2004). Scaffolding learning and co-constructing understandings. In A. Anning, J. Cullen \& M. Fleer, (Eds.), Early childhood education: Society and culture (pp.31-42). London: Sage.

Jordan, E. (2002). Masculinity in early childhood: Gender identity and the "civilising process". Journal of Australian Research in Early Childhood Education, 9(1), 27-36.

Jordan, E., Cowan, A., \& Roberts, J. (1995). Knowing the rules: Discursive strategies in young children's power struggles. Early Childhood Research Quarterly, 10, 339-358.

Kanpol, B. (1999). Critical pedagogy: An introduction ( $2^{\text {nd }}$ ed.). Westport, CT: Bergin \& Garvey.

Kaptelinin, V., \& Nardi, B. A. (2000). Activity theory: Basic concepts and applications. New York: ACM.

Katz, L. (1988). What should young children be doing? American Educator, 12(2), 28-33, 44-45.

Katz, L. (1994). What can we learn from Reggio Emilia? In C. Edwards, L. Gandini \& G. Foreman (Eds.), The hundred languages of children: The 
Reggio Emilia approach to early childhood education (pp.19-37).

Norwood, NJ: Ablex.

Katz, L. (2007). Standards of experience. Young Children, 62(3), 94-95.

Keddie, A. (2000). Emerging masculinities. Critical Pedagogy Networker, 13(3), $1-10$.

Keddie, A. (2003). Little boys: Tomorrow's macho lads. Discourse: Studies in the cultural politics of education, 24(3), 289-306.

Keesing-Styles, L. (2002). A critical pedagogy of early childhood education: The Aotearoa/New Zealand context. New Zealand Research in Early Childhood Education, 5, 109-122.

Keesing-Styles, L. (2003). The relationship between critical pedagogy and assessment in teacher education [Electronic Version]. Radical pedagogy, (5). Retrieved September 28, 2006, from http://radicalpedagogy.icaap.org/content/issue5_1/103_keesing-styles.html

Kennedy, A., \& Duncan, J. (2006). New Zealand children's spirituality in Catholic schools: Teachers' perspectives. International Journal of Children's Spirituality, 11(2), 281-292.

Kennedy, A., \& Ridgway, A. (2005). 'Catch the future" Literacy and numeracy pathways for young children: Voices from the field. Journal of Australian Research in Early Childhood Education, 12(1), 43-57.

Kincheloe, J. L. (2002). The complex politics of McDonald's and the new childhood: Colonizing kidworld. In G. Cannella \& J. L. Kincheloe (Eds.), Kidworld: Childhood studies, global perspectives, and education (pp.75121). New York: Peter Lang.

Kincheloe, J. L. (2004). Critical pedagogy primer. New York: Peter Lang.

Kincheloe, J. L., \& Berry, K. S. (2004). Rigour and complexity in educational research: Conceptualizing the bricolage. Maidenhead: Open University Press.

Kincheloe, J. L., \& McLaren, P. (2005). Rethinking critical theory and qualitative research. N. K. Denzin \& Y. S. Lincoln (Eds.), The Sage handbook of qualitative research ( $3^{\text {rd }}$ ed., pp.303-341). Thousand Oaks, CA: Sage.

King, R. (1978). All things bright and beautiful? A sociological study of infants' classrooms. Chichester: John Wiley \& Sons.

Knight, S. (2007). Maggie and the pirate: What role do circumstances play in judging moral rightness and wrongness? Critical and Creative Thinking, 15(2), 55-60. 
Kotzwinkle, W., \& Murray, G. (2001). Walter, the farting dog. Berkeley: Frog.

Krieg, S. (2003). Ethical research with children: Practices and possibilities.

Journal of Australian Research in Early Childhood Education, 10(1), 8192.

Kristensen, K. (2006). Boys' and girls' dramatic play: Gender and dramaturgy. Journal of Australian Research in Early Childhood Education, 13(1), 91101.

Kutti, K. (1996). Activity theory as a potential framework for human-computer interaction research. In B. A. Nardi (Ed.), Context and consciousness: Activity theory and human-computer interaction (pp.17-44). Cambridge, MA: MIT.

Kyratzis, A. (2004). Talk and interaction among children and the co-construction of peer groups and peer culture. Annual Review of Anthropology, 33, 625650.

Laevers, F. (2005). The curriculum as means to raise the quality of early childhood education: Implications for practice. European Early Childhood Education Research Journal, 13(1), 17-29.

Lather, P. (1991). Getting smart: Feminist research and pedagogy with/in the postmodern. London: Routledge.

Lawrence, R. (2004). Making sense of planning - A teacher's story. Early Education, (36), 15-19.

Lee, D. (2005). Early childhood social sciences: Social justice education or social engineering? ACE papers, (16), 54-66.

Lenz Taguchi, H. (2006). Reconceptualizing early childhood education:

Challenging taken-for-granted ideas. In J. Einarsdottir \& J. T. Wagner (Eds.), Nordic childhoods and early education: Philosophy, research, policy, and practice in Denmark, Finland, Iceland, Norway, and Sweden (pp.257-287). Greenwich, CT: Information Age Publishing.

Lewis, A. (2001). Research involving young children. In T. David (Ed.), Promoting evidence-based practice in early childhood education: Research and its implications (pp.253-271). Oxford: Elsevier Science.

Lewis, A., \& Lindsay, G. (Eds.). (2000). Researching children's perspectives. Buckingham: Open University Press.

Lewis, V., Kellett, M., Robinson, C., Fraser, S., \& Ding, S. (Eds.). (2004). The reality of research with children and young people. London: Sage. 
Lincoln, Y. S. (2002). Emerging criteria for quality in qualitative and interpretative research. In N. K. Denzin \& Y.S. Lincoln (Eds.), The qualitative inquiry reader (pp.327-345). Thousand Oaks, CA: Sage.

Lofdahl, A. (2002). Children' narratives in play - 'I put this rice pudding here, poisoned, so that Santa Claus will come and eat it!" Early Childhood Practice, 4(2), 38-47.

Loizou, E. (2005). Humour: A different kind of play. European Early Childhood Education Research Journal, 13(2), 97-109.

Longwell-Grice, H., \& Letts, W. J. (2001). Why doesn't the creed read "always be critical"? An examination of a liberal curriculum. Anthropology and Education Quarterly, 32(2), 191-214.

Lysklett, O. B. (2006, August). Outdoor play year-round in familiar surroundings. Paper presented at the $16^{\text {th }}$ European Early Childhood Research Association Conference, Reykjavik, Iceland.

MacNaughton, G. (2000). Rethinking gender in early childhood education. Sydney: Allen \& Unwin.

MacNaughton, G. (2001). Good teacher or feminist teacher?: Investigating the ethics of early childhood curriculum. In J. A. Jipson \& R. T. Johnson (Eds.), Resistance and representation: Rethinking childhood education (pp.59-76). New York: Peter Lang.

MacNaughton, G. (2003a). Eclipsing voice in research with young children. Australian Journal of Early Childhood, 28(1), 36-42.

MacNaughton, G. (2003b). Shaping early childhood: Learners, curriculum and contexts. Maidenhead: Open University.

MacNaughton, G. (2004). Exploring critical constructivist perspectives on children's learning. In A. Anning, J. Cullen \& M. Fleer (Eds.), Early childhood education: Society and culture (pp.43-54). London: Sage.

MacNaughton, G., Smith, K., \& Davis, K. (2007). Researching with children: The challenges and possibilities for building "child friendly" research. In J. A. Hatch (Ed.), Early childhood qualitative research (pp.167-184). New York: Routledge.

MacNaughton, G., \& Williams, G. (2004). Techniques for teaching young children: Choices in theory and practice $\left(2^{\text {nd }}\right.$ ed.). Frenchs Forest, NSW: Pearson Prentice Hall.

Malaguzzi, L. (1993). For an education based on relationships. Young Children, 49(1), 9-12. 
Mandell, N. (1988). The least-adult role in studying children. Journal of Contemporary Ethnography, 16(4), 433-467.

Manning, S. (2008). Playcentre parents-as-educators: Links between background experiences and teaching practice. Unpublished master's thesis, Victoria University, Wellington.

Margolis, E. E., Soldatenko, M., Acker, S., \& Gair, M. (2001). Peekaboo: Hiding and outing the curriculum. In E. E. Margolis (Ed.), The hidden curriculum in higher education (pp.1-19). New York: Routledge.

Martin, K. A. (1998). Becoming a gendered body: Practices of preschools. American Sociological Review, 63(4), 494-511.

Matusov, E. (2007). In search of 'the appropriate' unit of analysis for sociocultural research. Culture and Psychology, 13(3), 307-333.

May, H. (2001). Politics in the playground: The world of early childhood in postwar New Zealand. Wellington: Bridget Williams Books with New Zealand Council for Educational Research.

Mayall, B. (2000). Conversations with children: Working with generational issues. In P. Christensen \& A. James (Eds.), Research with children: Perspectives and practices (pp.120-135). London: Falmer.

Mayall, B. (2002). Towards a sociology for childhood: Thinking from children's lives. Buckingham: Open University Press.

Maynard, T. (2007). Forest Schools in Great Britain: An initial exploration. Contemporary Issues in Early Childhood, 8(4), 320-330.

McArdle, F. (2005). Teaching notes. In S. Ryan \& S. Grieshaber (Eds.), Practical transformations and transformational practices: Globalization, postmodernism, and early childhood education (pp.59-92). Amsterdam: Elsevier.

McCadden, B. M. (1998). It's hard to be good: Moral complexity, construction, and connection in a kindergarten classroom. New York: Peter Lang.

McCutcheon, G. (1997). Curriculum and the work of teachers. In D. J. Flinders \& S. J. Thornton (Eds.), The curriculum studies reader (pp.188-197). New York: Routledge.

McDonald, G., Le, H., Higgins, J., \& Podmore, V. (2005). Artifacts, tools and classrooms. Mind, Culture and Activity, 12(2), 113-127.

McLaren, P. (1994). Multiculturalism and the post-modern critique: Toward a pedagogy of resistance and transformation. In H. A. Giroux \& P. McLaren 
(Eds.), Between borders: Pedagogy and the politics of cultural studies (pp.212-220). London: Routledge.

McLaren, P. (1998). Life in schools: An introduction to critical pedagogy in the foundations of education ( $3^{\text {rd }}$ ed.). New York: Longmans.

McLaren, P. (2007). Life in schools: An introduction to critical pedagogy in the foundations of education ( $5^{\text {th }}$ ed.). Boston: Pearson / Allyn \& Bacon.

McLeod, L. S. (2002). Leadership and management in early childhood centres: A qualitative case study. Unpublished doctorate thesis, Massey University, Palmerston North.

McNaughton, S. (1996). Co-constructing curricula: A comment of two curricula (Te Whāriki and the English curriculum) and their developmental bases. New Zealand Journal of Educational Studies, 31(2), 189-196.

Meade, A. (Ed.). (2005). Catching the waves: Innovation in early childhood education. Wellington: New Zealand Council for Educational Research.

Meade, A. (Ed.). (2006). Riding the waves: Innovation in early childhood education. Wellington: New Zealand Council for Educational Research.

Meade, A. (Ed.). (2007). Cresting the waves: Innovation in early childhood education. Wellington: New Zealand Council for Educational Research.

Meade, A., \& Cubey, P. (2008). Thinking children: Learning about schema. Maidenhead: McGraw Hill/Open University Press.

Mearns, H. (n.d.). As I was going up the stair. Retrieved January 28, 2009, from http://www.nationmaster.com/encyclopedia/Hughes-Mearns

Middleton, S., \& May, H. (1997). Teachers talk teaching 1915-1995: Early childhood, schools and teachers' colleges. Palmerston North: Dunmore Press.

Millei, Z. J. (2005). The discourse of control: Disruption and Foucault in an early childhood classroom. Contemporary Issues in Early Childhood, 6(2), 128139.

Ministry of Education. (1993). New Zealand curriculum framework: Te anga marautanga o Aotearoa. Wellington: Learning Media.

Ministry of Education. (1993). Te whāriki: Early childhood curriculum. He whāriki mātauranga mō ngā mokopuna o Aotearoa. Draft guidelines for developmentally appropriate programmes in early childhood services. Wellington: Learning Media. 
Ministry of Education. (1996). Te whäriki: Early childhood curriculum. He whāriki mātauranga mō ngā mokopuna o Aotearoa. Wellington: Learning Media.

Ministry of Education. (2002). Pathways to the future: Ngā huarahi arataki. Wellington: Learning Media.

Ministry of Education. (2004a). Basic equipment list for licensed early childhood centres. Wellington: Learning Media.

Ministry of Education. (2004b). Kei tua o te pae Assessment for learning: Early childhood exemplars. Wellington: Learning Media.

Murfin, B., \& Butterworth, D. (1999). Researching children: The woes and joys of observing and interviewing four-year-olds. Australian Journal of Early Childhood, 24(2), 6-9.

Mutch, C. (2003). One context, two outcomes: A comparison of Te Whāriki and the New Zealand Curriculum Framework. In J. Nuttall (Ed.), Weaving Te Whäriki: Aotearoa New Zealand's early childhood curriculum document in theory and practice. Wellington: New Zealand Council for Educational Research.

Nardi, B. A. (1996). Activity theory and human-computer interaction. In B. A. Nardi (Ed.), Context and consciousness: Activity theory and humancomputer interaction (pp.7-16). Cambridge, MA: MIT Press.

Nespor, J. (1998). The meanings of research: Kids as subjects and kids as inquirers. Qualitative Inquiry, 4(3), 369-389.

New, R. S., Mardell, B., \& Robinson, D. (2005). Early childhood education as risky business: Going beyond what's "safe" to discovering what's possible. Early Childhood Research and Practice, 7(2), 1-18.

Nimmo, J. (2008). Young children's access to real life: An examination of the growing boundaries between children in child care and adults in the community. Contemporary Issues in Early Childhood, 9(1), 3-13.

Norberg, K. (2006). Morality at the margins: A silent dimension of teaching and learning. Journal of Curriculum Studies, 38(2), 189-204.

Norris, J. (2001). Crossing the threshold: Masculinities and the transition to school. Wellington: Institute for Early Childhood Studies, Victoria University of Wellington.

Nutbrown, C. (2006a). Key concepts in early childhood education and care. London: Sage. 
Nutbrown, C. (2006b). Threads of thinking: Young children learning and the role of early education. London: Sage.

Nuttall, J. (2002). Negotiating the meaning of 'curriculum': Can we awaken the 'sleeping beauty'? Early Education, (28), 5-9.

Nuttall, J. (2003). Weaving Te Whāriki: Aotearoa New Zealand's early childhood curriculum document in theory and practice. Wellington: New Zealand Council for Educational Research.

Nuttall, J. (2004). Why don't you ask someone who cares? Teacher identity, intersubjectivity, and curriculum negotiation in a New Zealand childcare centre. Unpublished doctorate thesis, Victoria University, Wellington.

Oldfield, L. (2001). Free to learn: Introducing Steiner Waldorf early childhood education. Stroud: Hawthorn Press.

Oldridge, L. (2009). Examining early childhood teachers' perceptions of ICT. Journal of Australian Research in Early Childhood Education, 15(2), 5364.

Olds, A. R. (2001). Day care centers: Design and construction. New York: McGraw Hill.

O'Rourke, M. (1988). Curriculum objectives in early childhood education. Wellington: New Zealand Council for Educational Research.

Pacini-Ketchabaw, V. (2007). Child care and multiculturalism: A site of governance marked by flexibility and openness. Contemporary Issues in Early Childhood, 8(3), 222-232.

Paley, V. G. (1984). Boys and girls: Superheroes in the doll corner. Chicago: University of Chicago Press.

Paley, V. G. (1986). Mollie is three: Growing up in school. Chicago: University of Chicago Press.

Paley, V. G. (1990). The boy who would be a helicopter: The uses of storytelling in the classroom. Cambridge, MA: Harvard University Press.

Paley, V. G. (1992). You can't say you can't play. Cambridge, MA: Harvard University Press.

Paley, V. G. (1995). Kwanzaa and me: A teacher's story. Cambridge, MA: Harvard University Press.

Paley, V. G. (2001). In Mrs Tully's room: A childcare portrait. Cambridge, MA: Harvard University Press.

Paley, V. G. (2004). A child's work: The importance of fantasy play. Chicago: University of Chicago Press. 
Patton, M.Q. (2002). Qualitative research and evaluation methods $\left(3^{\text {rd }} \mathrm{ed}.\right)$. Thousand Oaks, CA: Sage.

Pinar, W. F., Reynolds, W. M., Slattery, P., \& Taubman, P. M. (1995). Understanding curriculum: An introduction to the study of historical and contemporary curriculum discourses. New York: Peter Lang.

Podmore, V., Sauvao, L. M., \& Mapa, L. (2003). Sociocultural perspectives on transition to school from Pacific Islands early childhood centres. International Journal of Early Years Education, 11(1), 33-42.

Podmore, V. N., \& May, H. (2003). "The child's questions": Narrative explorations of infants' experiences of Te Whäriki. Journal of Australian Research in Early Childhood Education, 10(1), 69-80.

Pole, C., \& Morrison, M. (2003). Ethnography for education. Berkshire: Open University Press.

Popper, K. (1994). The myth of framework: In defence of science and rationality. London: Routledge.

Pramling Samuelsson, I. (2000). Teaching and learning in preschool and the first years of elementary school in Sweden. In J. Einarsdottir \& J. T. Wagner (Eds.), Nordic childhoods and early education: Philosophy, research, policy, and practice in Denmark, Finland, Iceland, Norway, and Sweden (pp.101-131). Greenwich, CT: Information Age Publishing.

Pramling Samuelsson, I., Sheridan, S., \& Williams, P. (2006). Five preschool curricula: Comparative perspective. International Journal of Early Childhood, 38(1), 11-31.

Prescott, E. (2008). The physical environment: A powerful regulator of experience. Exchange, (180), 34-37.

Prochner, L. (2001). "The proof of the home is the nursery": An American proverb revisited. In J. A. Jipson \& R. T. Johnson (Eds.), Resistance and representation: Rethinking childhood education (pp.205-221). New York: Peter Lang.

Prout, A. (2005). The future of childhood. New York: RoutledgeFalmer.

Prout, A., \& James, A. (1990). A new paradigm for the sociology of childhood? Provenance, promise and problems. In A. James, \& Prout, A. (Ed.), Constructing and reconstructing childhood: Contemporary issues in the sociology of childhood (pp.7-34). London: Falmer.

Provenzo, E. F. (2002). Teaching, learning and schooling: A twenty-first century perspective. Boston: Allyn \& Bacon. 
Ramsey, K., Breen, J., Sturm, J., Lee, W., \& Carr, M. (2006). Strengthening learning and teaching using ICT: Roskill South Kindergarten Centre of Innovation 2003-2006 Final research report. Wellington: Ministry of Education.

Ramsey, K., Sturm, J., Breen, J., Lee, W., \& Carr, M. (2007). Weaving ICTs into Te Whäriki at Roskill South Kindergarten. In A. Meade (Ed.), Cresting the waves: Innovation in early childhood education (pp.29-36). Wellington: New Zealand Council for Educational Research.

Rhedding-Jones, J. (2005). Questioning diversity. In N. Yelland (Ed.), Critical issues in early childhood education (pp.131-145). Maidenhead: Open University Press.

Rinaldi, C. (2006). In dialogue with Reggio Emilia: Listening, researching and learning. London: Routledge.

Ritchie, J. (2001). Reflections on collectivism in early childhood teaching in Aotearoa/New Zealand. In S. Grieshaber \& G. Cannella (Eds.), Embracing identities in early childhood education: Diversity and possibilities (pp.133-147). New York: Teachers College Press.

Ritchie, J. (2003). Te Whāriki as a potential lever for bicultural development. In J. Nuttall (Ed.), Weaving Te Whāriki: Aotearoa New Zealand's early childhood curriculum document in theory and practice. Wellington: New Zealand Council for Educational Research.

Ritchie, J. (2005). Implementing Te Whāriki as postmodernist practice: A perspective from Aotearoa/New Zealand. In S. Ryan \& S. Grieshaber (Eds.), Practical transformations and transformational practices: Globalization, postmodernism, and early childhood education (pp.109136). Amsterdam: Elsevier.

Rivalland, C. M. P. (2007). When are beliefs just 'the tip of the iceberg'? Exploring early childhood professionals' beliefs and practices about teaching and learning. Australian Journal of Early Childhood, 32(1), 3037.

Robertson, J. (2006). Reconsidering our images of children: What shapes our educational thinking? In A. Fleet, C. Patterson \& J. Robertson (Eds.), Insights: Behind pedagogical documentation (pp.37-54). Castle Hill: Pademelon.

Robinson, K. H. (2005). 'Queerying' gender: Heteronormativity in early childhood education. Australian Journal of Early Childhood, 30(2), 19-28. 
Robson, S. (2006). Developing thinking and understanding in young children. London: Routledge.

Robson, S., \& Hargreaves, D. J. (2005). What do early childhood practitioners think about young children's thinking? European Early Childhood Education Research Journal, 13(1), 81-96.

Rogoff, B. (2003). The cultural nature of human development. New York: Oxford University Press.

Roopnarine, J. L., \& Johnson, J. E. (2005). Approaches to early childhood education ( $4^{\text {th }}$ ed.). Upper Saddle River, NJ: Pearson.

Rosenthal, M. K. (2003). Quality in early childhood education and care: A cultural context. European Early Childhood Education Research Journal, 11(2), 101-116.

Russell, D. R. (2004). Looking beyond the interface: Activity theory and distributed learning. In H. Daniels \& A. Edwards (Eds.), The RoutledgeFalmer reader in psychology of education (pp.309-323). London: RoutledgeFalmer.

Rutanen, N. (2006, August). Two year old children as co-constructors of culture. Paper presented at the $16^{\text {th }}$ European Early Childhood Research Association Conference, Reykjavik, Iceland.

Ryan, M., \& Stower, L. (1998). A vision of the whole child: The significance of religious experiences in early childhood. Australian Journal of Early Childhood, 23(1), 1-4.

Ryan, S., \& Grieshaber, S. (2004). It's more than child development: Critical theories, research, and teaching young children. Young Children, 59(6), 44-52.

Ryan, S., \& Ochsner, M. (1999). Traditional practices, new possibilities: Transforming dominant images of early childhood teachers. Australian Journal of Early Childhood, 24(4), 14-20.

Sandester, E. B. (2006, August). Children's risky play in the Norwegian "barnehage": A pilot study. Paper presented at the $16^{\text {th }}$ European Early Childhood Research Association Conference, Reykjavik, Iceland.

Sanson, A. (2006). The body in early childhood: Revisioning dance. The First Years: Ngā Tau Tuatahi New Zealand Journal of Infant and Toddler Education, 8(2), 25-28. 
Sanson, A. (2007). Is the body an endangered species? Reconceptualizing the body in early childhood education. The First Years: Ngā Tau Tuatahi New Zealand Journal of Infant and Toddler Education, 9(2), 13-16.

Schwall, C. (2005). The atelier environment and materials. In L. Gandini, L. Hill, L. Cadwell \& C. Schwall (Eds.), In the spirit of the studio: Learning from the atelier of Reggio Emilia (pp.16-31). New York: Teachers College Press.

Scutt, J. (1992). Practice and professionalism: A positive ethos for early childhood. In B. Lambert (Ed.), Changing faces: The early childhood profession in Australia (pp.31-45). Watson, ACT: Australian Early Childhood Association.

Siegler, R. S., \& Alibali, M. (2004). Children's thinking ( $4^{\text {th }}$ ed.). Upper Saddle River, NJ: Pearson Prentice Hall.

Silin, J. (1995). Sex, death, and the education of children: Our passion for ignorance in the age of AIDS. New York: Teachers College Press.

Silin, J. (1997). HIV/AIDS education: Toward a collaborative curriculum. In D. J. Flinders \& S. J. Thornton (Eds.), The curriculum studies reader (pp.224-246). New York: Routledge.

Simmons, H., Schimanski, L., McGarva, P., Haworth, P., \& Cullen, J. (2007). A bilingual intercultural setting: What have we discovered?. In A. Meade (Ed.), Cresting the waves: Innovation in early childhood education (pp.6370). Wellington: New Zealand Council for Educational Research.

Sims, M. (2000). Young children's conflict behaviour in child care. Journal of Australian Research in Early Childhood Education, 7(1), 78-86.

Singer, E. (1996). Prisoners of the method: Breaking open the child-centred pedagogy in day care centres. International Journal of Early Years Education, 4(2), 28-40.

Singer, E. (2003). The logic of young children's (nonverbal) behaviour. In B. van Oers (Ed.), Narratives of childhood: Theoretical and practical explorations of the innovation of early childhood education (pp.68-80). Amsterdam: VU University.

Singer, E. (2005). The liberation of the child. Early Child Development and Care, 175(6), 611-620.

Singer, E., \& Hännikäinen, M. (2002). The teacher's role in territorial conflicts of 2- to 3-year old children. Journal of Research in Childhood Education, 17(1), 5-18. 
Siraj-Blatchford, I., \& Siraj-Blatchford, J. (2001). An ethnographic approach to researching young children's learning. In G. MacNaughton, S. A. Rolfe \& I. Siraj-Blatchford (Eds.), Doing early childhood research: International perspectives on theory and practice (pp.193-207). Maidenhead: Open University Press.

Skattebol, J., \& Ferfolja, T. (2007). Voices from an enclave: Lesbian mothers' experiences of child care. Australian Journal of Early Childhood, 32(1), $10-18$.

Skelton, A. (1997). Studying hidden curricula: Developing a perspective in the light of postmodern insights. Curriculum Studies, 5(2), 177-193.

Skinner, D., Bryant, D., Coffman, J., \& Campbell, F. (1998). Creating risk and promise: Children's and teachers' co-constructions in the cultural world of kindergarten. The Elementary School Journal, 98(4), 297-401.

Smith, A., Duncan, J., \& Marshall, K. (2005). Children's perspectives on their learning: Exploring methods. Early Child Development and Care, 175(6), 473-487.

Smith, A. B., \& Swain, D. A. (1988). Childcare in New Zealand: People, programmes, politics. Wellington: Allen \& Unwin/ Port Nicholson Press.

Smith, A. B., \& Taylor, N. J. (2000). The sociocultural context of childhood: Balancing dependency and agency. In A. B. Smith, N. J. Taylor \& M. M. Gollop (Eds.), Children's voices: Research, policy and practice (pp.1-18). Auckland: Pearson Educational.

Smith, A. B., Taylor, N. J., \& Gollop, M. M. (Eds.). (2000). Children's voices: Research, policy and practice. Auckland: Pearson Educational.

Somerset, G. L. (1967). I play and I grow. Auckland: New Zealand Play Centre Federation.

Sorin, R. (2003). Research with children: A rich glimpse into the world of childhood. Australian Journal of Early Childhood, 28(1), 31-35.

Soto, L. D., \& Swadener, B. B. (2002). Toward liberatory early childhood theory, research and praxis: Decolonizing a field. Contemporary Issues in Early Childhood, 3(1), 38-66.

Soto, L. D., \& Swadener, B. B. (Eds.). (2005). Power and voice in research with children. New York: Peter Lang.

Stephen, C., \& Plowman, L. (2002). ICT in pre-school: A 'benign addition'?: A review of the literature on ICT in pre-school settings. Dundee: Learning and Teaching Scotland. 
Stephenson, A. (1998). Opening up the outdoors: A reappraisal of young children's outdoor experiences. Unpublished master's thesis, Victoria University, Wellington.

Stephenson, A. (2003). Physical risk-taking: Dangerous or endangered? Early Years: An International Journal of Research and Development, 23(1), 3543.

Stephenson, A. (2008, January). Another way of looking: Considering adult roles from children's perspectives. Paper presented at the Australian Early Childhood Research Symposium, Melbourne.

Stephenson, A. (2009). Can teaching be invisible? Considering children's experience of the roles of teachers. Journal of Australian Research in Early Childhood Education, 15(2), 1-13.

Stephenson, A. (2009a). Horses in the sandpit: Photography, prolonged involvement and 'stepping back' as strategies for listening to children's voices. Early Child Development and Care,

Stephenson, A. (2009b). Stepping back to listen to Jeff: Conversations with a 2year-old. Young Children,

Stover, S. (2003). The history and significance of the Playcentre movement. In S. Stover (Ed.), Good clean fun: New Zealand's Playcentre Movement (Rev. ed., pp.1-21). Auckland: New Zealand Playcentre Federation.

Strandell, H. (2000). What is the use of children's play: Preparation or social participation? In H. Penn (Ed.), Early childhood services: Theory, policy and practice (pp.147-157). Buckingham: Open University Press.

Sumison, J. (2003). Researching with children: Lessons in humility, reciprocity, and community. Australian Journal of Early Childhood, 28(1), 18-23.

Surtees, N. (2003). Unravelling the woven mat: Queering the whāriki. Waikato Journal of Education, 9, 143-153.

Tait, G. (2001). 'No touch' policies and the government of risk. In A. Jones (Ed.), Touchy subjects: Teachers touching children (pp.39-49). Dunedin: University of Otago Press.

Taylor, A., \& Richardson, C. (2005). 'Home renovations, border protection and the hard work of belonging'. Journal of Australian Research in Early Childhood Education, 12(1), 93-100.

Te One, S. (2003). The context for Te Whäriki: Contemporary issues of influence. In J. Nuttall (Ed.), Weaving Te Whāriki: Aotearoa New Zealand's early 
childhood curriculum document in theory and practice (pp.17-48).

Wellington: New Zealand Council for Educational Research.

Te One, S. (2004). Children's rights and early childhood policy: Impacts and influences. New Zealand Annual Review of Education, 14, 171-193.

Te One, S. (2005). Children's rights and early childhood policy: A New Zealand story. European Early Childhood Education Research Journal, 13(2), 25-39.

Te One, S. (2007). Participatory-research methods with young children:

Experiences from the field. Early Childhood Folio, 11, 21-25.

Te One, S. (in preparation). Interwoven, interrelated, interdependent: Perceptions of children's rights in three early childhood settings. Unpublished doctorate thesis, Victoria University, Wellington.

Test, J. (2006). Infant and toddler teachers as transmitters of culture. International Journal of Early Childhood, 38(1), 47-63.

Thomas, J. (1993). Doing critical ethnography. Newbury Park: Sage.

Thompson, C. M. (2003). Kinderculture in the art classroom: Early childhood art and the mediation of culture. Studies in Art Education, 44(2), 135-146.

Tobin, J. J. (1997). Playing doctor in two cultures. In J. J. Tobin (Ed.), Making a place for pleasure in early childhood education (pp.119-158). New Haven: Yale University.

Tovey, H. (2008, July). The dangers of safety: Perceptions of risk and challenge in play outdoors. Paper presented at the International Froebel Society Third Biennial Conference, Boston.

van Wijk, N., \& Wilton Playcentre community (2007). Wilton Playcentre: A community of learners, tall and short. In A. Meade (Ed.), Cresting the waves: Innovation in early childhood education (pp.56-62). Wellington: New Zealand Council for Educational Research.

Viruru, R. (2001). Colonized through language: The case of early childhood education. Contemporary Issues in Early Childhood, 2(1), 31-47.

Viruru, R., \& Cannella, G. S. (2001). Postcolonial ethnography, young children, and voice. In S. Grieshaber \& G. Cannella (Eds.), Embracing identities in early childhood education: Diversity and possibilities (pp.158-172). New York: Teachers College Press.

Waksler, F. C. (1991). Studying children: Phenomenological insights. In F.C. Waksler (Ed.), Studying the social worlds of children: Sociological readings (pp.60-69). London: Falmer. 
Walkerdine, V. (1992). Progressive pedagogy and political struggle. In C. Luke \& J. Gore (Eds.), Feminisms and critical pedagogy (pp.15-24). New York: Routledge.

Walsh, P. (1996). Best practice guidelines in early childhood physical environments. Ashfield, NSW: NSW Department of Community Services.

Watson, J. (2005). Superheroes plus teachers - an unbeatable team! Early Education, (38), 35-40.

Wilks, A. (1993). Assessment of children in kindergartens and childcare centres. Wellington: Ministry of Education.

Williams, L. R., \& Fromberg, D. P. (Eds.). (1992). Encyclopaedia of early childhood education. New York: Garland.

Wiltz, N. W., \& Klein, E. L. (2001). "What do you do in child care?" Children's perceptions of high and low quality classrooms. Early Childhood Research Quarterly, 16, 209-236.

Wohlwend, K. (2007). Friendship meeting or blocking circle? Identities in the laminated spaces of a playground conflict. Contemporary Issues in Early Childhood, 8(1), 73-88.

Wood, E. (2004). Developing a pedagogy of play. In A. Anning, J. Cullen \& M. Fleer (Eds.), Early childhood education: Society and culture (pp.1930). London: Sage.

Wood, E., \& Attfield, J. (2005). Play, learning and the early childhood curriculum ( $2^{\text {nd }}$ ed.). London: Paul Chapman.

Woodrow, C. (1999). Revisiting images of the child in early childhood education: Reflections and considerations. Australian Journal of Early Childhood, 24(4), 7-12.

Woodrow, C., \& Brennan, M. (2001). Interrupting dominant images: Critical and ethical issues. In J. A. Jipson \& R. T. Johnson (Eds.), Resistance and representation: Rethinking childhood education (pp.23-43). New York: Peter Lang.

Woodrow, C., \& Fasoli, L. (1998). Change and criticism: Thinking critically in early childhood collaboration. Australian Journal of Early Childhood, $23(1), 40-44$

Wright, L. (2003). Living the early childhood curriculum: Five days in family day care settings. Unpublished master's thesis, Victoria University, Wellington. 
Wyness, M. (2006). Childhood and society: An introduction to the sociology of childhood. New York: Palgrave MacMillan.

Yelland, N., \& Kilderry, A. (2005). Against the tide: New ways in early childhood education. In N. Yelland (Ed.), Critical issues in early childhood education (pp.243-248). Maidenhead: Open University. 
APPENDICES 
[VUW Letterhead]

\section{Exploring the scope and boundaries of the curriculum}

In this research project I am interested in exploring the scope, and the boundaries of the curriculum that is experienced and enacted by children in an early childhood setting. The definition of curriculum in Te Whariki is

...the sum total of the experiences, activities, and events, whether direct or indirect, which occur within an environment designed to foster children's learning and development.

(Ministry of Education, 1996, p.10)

As early childhood teachers, we spend a great deal of time discussing and reflecting on the "direct" experiences, activities and events that are planned for children. In contrast, relatively little time is spent considering the "indirect" aspects. While I want to record details of the "direct" learning, I want to move beyond it and focus on the other things that children are, or are not learning while they are in a centre.

- Some of this less obvious learning will be conveyed in the environment of the centre, the kind of equipment available, in the way the centre is organized, and in things that teachers do and say. Collectively, these aspects have been called "the hidden curriculum".

- Some of the less obvious learning will also happen between children, and therefore peer group interactions will also be a focus.

- Finally, I want to explore the boundaries of the enacted curriculum, and consider the "null curriculum". These are the things that society, or the profession, or the teachers in the centre, have defined as not relevant or appropriate to the curriculum

In all stages of this research project, my primary focus is to capture the children's perspective, and the children's voices. I want to describe the curriculum, as it is enacted, from their perspective. I will therefore not be focusing on the teachers' perspectives.

While I plan to do this research in a single setting, my aim is to raise questions and provoke discussion about curriculum that is relevant to the whole of the early childhood profession. I am envisaging that the particular setting and children will provide me with illustrations of many aspects of early childhood education that are universally accepted. 


\section{Details about the research process}

This research project is being undertaken for my Ph.D. through Victoria University. My thesis supervisors are Professor Helen May (-----) and Dr Jim Neyland (----). The research has been approved by the Faculty of Education ethics subcommittee.

The sources of information that I plan to use in this research project are as follows. Initially I will collect information from:

- Children's portfolios

- Teachers' planning documentation

- Centre newsletters/information for families/whānau

- Photographs of the environment.

As I develop a rapport with children I will begin gathering information from them. I plan to try various strategies, and find what seems most appropriate:

- Observing children (at times when they agree to it)

- Talking with children, either singly or in groups, as it happens within the flow of the session, and only with their agreement

- Giving children the opportunity to take photographs within the centre as a way of identifying what for them are significant areas/equipment

- Using photographs (taken by me, or by children) as prompts for conversations with children (at times that they agree to)

- Completing a questionnaire with individual children using smiling and frowning faces (only if and when they volunteer) as a way of exploring if, and what, they consider they are learning in the centre

- Audio recording of children' conversations (with children's agreement)

- Child-led "tours of the centre".

Towards the end of the data gathering I may develop a few case studies of particular children, if this seems appropriate. This would be done with the knowledge of the educators, and of the parents/caregivers. Semi-structured interviews with the parents/ of these children would $b$ requested to gather additional information on children's of-of-centre interests.

It is anticipated that the data that I gather will be analysed using a software package for qualitative data analysis called NVivo, which involves coding data and searching for underlying patterns. All the data will be stored under password on my home computer, and files will be destroyed 3 years after the presentation of the thesis.

I would be very happy to come and talk to you in more detail about my proposed research

Alison Stephenson

---- day

---- evg 


\section{Appendix 2: Permission letter to Centre Management}

(There were only minor differences in the wording in permission letters for adults working in the centre and for parents)

[VUW Letterhead]

June 2005

\section{Kia ora}

I am planning a research project that looks at the scope and boundaries of what actually constitutes curriculum in one early childhood centre setting, and which will focus largely on the perspectives of the children. The flier I have included gives more information about the kinds of things that I am interested in finding out. This project is for my Ph.D., which I am doing through Victoria University, with Professor Helen May and Dr Jim Neyland as my supervisors. The project has received ethical approval from the School of Education Ethics Sub-committee of Victoria University. I want to focus on what happens in one childcare centre that caters for a mixed age range of children. I believe ... would be ideal and I wondered if you, and the teachers, would consider this request.

I have included an information sheet about the research, and also a copy of the full thesis proposal that has been approved by Victoria University. There are a few points that I would like to clarify.

\section{The time in the centre}

I would like to complete the information-gathering in the centre between July and late November 2005, as I am able to take leave from my position at the University for the second half of the year. Initially I would like to visit the centre for a few hours over several days at the time when I send out the consent forms to families and children. Having this time in the centre will help me to become familiar with the routines so I can plan my research in ways that fit in with these. It will also give the children and their families a chance to meet me, and to ask any questions they may have. 
While it is not easy to predict how long data gathering will take in an ethnographic research, I anticipate that once I begin the project I would be in the centre relatively frequently for an initial period of six weeks, and usually staying for 3 or 4 hours at a time. After that, as I begin more in-depth analysis, I would visit less frequently, and I plan to have the data gathering completed by the end of November.

\section{The process of gathering information}

When I am visiting the centre I would like at first to gather information through reading documents such as children's portfolios, teachers' planning documentation and centre newsletters. As I get to know the children and teachers, I would spend time observing children, taking notes and photographs, talking with children and perhaps occasionally tape recording. I understand the challenges of working in a centre, and am mindful of the need for my activities to fit within the daily flow of the session, and for the research not to be a burden on either the teachers or the children.

\section{Keeping the participants informed}

I will take a number of steps to keep all the participants informed about the ongoing project. With the children's permission, my observations, and transcripts will be available to all the teachers. Families, teachers and the Management Committee will receive a regular newsletter about the project. Each family can request a copy of the information that relates to their child/children, with the proviso that I will check with their child/children first before handing it over. Each child will receive copies of photographs in which they are included. The centre will receive a copy of the full final thesis, and families can receive a summary of the findings if they wish.

\section{The process of seeking consent from participants}

I will approach each staff member individually, to ask them to consider participating, with a written permission form to complete. I will also approach each family, and each child whose family feels they are old enough to give their own consent. Any individual or family can withdraw from the study at any time, and the centre can also ask that the research cease at any stage of the data gathering. 


\section{Confidentiality}

In order to keep the information that I gather secure, it will be kept in a locked filing cabinet at my home, and under password on my home computer and my computer at Victoria University. I will also ensure that the identity of the centre, and the participants, remains confidential. In the thesis, and in any conference presentations or written papers that arise from this study, the centre and all participants will be identified by pseudonyms that they have chosen.

I have enclosed a consent form for you to complete, and an addressed envelope for you to return it to me. If you have any questions I would be very happy to come and talk with you. I can be contacted most easily at home in the evening $(\operatorname{xxxxxx})$.

If you have questions for the supervisors of my research, Professor Helen May can be contacted at Otago University on xxxxxx and Dr Jim Neyland can be contacted at Victoria University ( $x x x x x x)$. It is possible that my Ph.D. will be transferred to Otago University as Helen May has just been appointed there. I will keep you informed of any changes.

I look forward to hearing from you
Alison Stephenson
Senior lecturer
Victoria University of Wellington 


\section{Appendix 3: Consent form for Centre Management}

[VUW Letterhead]

\section{CONSENT FORM FOR MANAGEMENT COMMITTEE}

Please tick each of the following if you agree:

We agree that Centre can participate in the

YES NO research conducted by Alison Stephenson.

We have been provided with adequate information relating to the nature and objectives of this research project, we have understood that information, and have YES NO been given the opportunity to seek further information from the researcher.

We understand that the centre can ask to withdraw from the research at any stage before the start of data analysis without needing to give a reason.

We understand that any participant, adult or child, has the right to withdraw from the research at any stage before the YES NO final data analysis without needing to give a reason.

We understand that the identity of the centre, and of all the participants will be kept confidential, and that pseudonyms will be used in the thesis, in conference and teaching presentations, and in any published papers. 
We understand that the information gathered will be used only for reporting this research project in the thesis, in conference presentations, in teaching sessions relating to this topic, and in published academic YES NO papers.

We understand that all the data gathered will be destroyed three years after the thesis is presented.

We understand that any member of the Management Committee, any staff member, or any parent or child is able to approach Alison at any stage of the data YES NO gathering, if they have questions or concerns.

We understand that we will receive regular newsletters that inform us of the progress of the data gathering, and that the YES NO centre will receive a full copy of the final thesis.

We would like to receive a summary of the final research report when it is completed.

Signed:

Dated: 


\section{Appendix 4: Consent form for adults in the Centre}

[VUW Letterhead]

\section{CONSENT FORM FOR ADULTS WORKING IN THE CENTRE}

Please tick each of the following if you agree:

I have been provided with adequate information relating to the nature and objectives of this research project, I have understood that information, and have been given the opportunity to seek further information from the researcher needed.

I understand that any participant, adult or child, has the right to withdraw from the research at any stage before the start of data analysis without needing to give a reason.

I understand that the identity of the centre, and of all the participants will be kept confidential, and that pseudonyms will be used when referring to individuals in the thesis, in conference and teaching presentations, and in published papers that report on this research.

I understand that the information gathered will be used only for reporting this research project in the thesis, in YES NO conference presentations, in teaching sessions relating to this topic, and in published academic papers.

I understand that all the data gathered will be destroyed YES NO three years after the thesis is presented. 
I understand that any member of the Management committee, any staff member, or any parent or child is able to approach the researcher at any stage of the data gathering, if they have questions or concerns.

I understand that I will receive regular newsletters that inform me of the progress of the data gathering, and that the centre will receive a full copy of the final thesis.

YES NO

I understand that I can request copies of all information gathered which relates to

YES NO me

I understand that the adults who work in the centre will all have a chance to comment on the information that relates YES NO collectively to them.

I understand that the teachers can have request to have copies of the information that is gathered about the children. (I understand that if a child has signed their own participation form, they will be asked for their verbal agreement before this information is given to teachers.)

I would like to receive a summary of the final research report when it is completed.

YES NO

I agree to be a participant in the research conducted by Alison YES NO Stephenson

Signed:

Dated: 


\section{Appendix 5: Consent form for parents/guardians}

\section{CONSENT FORM FOR PARENTS/FAMILIES/WHANAU}

Please tick each of the following if you agree:

I have been provided with adequate information relating to the nature and objectives of this research project, I have understood that information, and have been given the opportunity to seek further information from the researcher.

I understand that any participant, adult or child, has the right to withdraw from the research at any stage before the start of data analysis without needing to give a reason.

I understand that the identity of the centre, and of all the participants will be kept confidential, and that pseudonyms will be used in the thesis, in conference and teaching presentations, and in any published papers.

I understand that the information gathered will be used only for reporting this research project in the thesis, in conference presentations, in teaching sessions relating to this topic, and in published academic papers.

I understand that all the data gathered will be destroyed three YES NO years after the thesis is presented. 
I understand that any member of the Management Committee, any staff member, and any parent or child is able to approach the researcher at any stage of the data YES NO gathering, if they have questions or concerns.

I understand that I will receive regular newsletters that inform me of the progress of the data gathering, and that the centre YES NO will receive a full copy of the final thesis.

I understand that I can request copies of all information gathered which relates to my child/children. (I understand YES NO that my child/children will be asked for their verbal agreement before this information is given to me.)

I would like to receive a summary of the final research report when it is completed

I give permission for my child / children:

to be a participant / participants in the research conducted by Alison Stephenson at Centre.

Signed:

Dated: 
Please tick one of the following:

\section{EITHER}

I have included an assent form/s completed by my child/children with my help

YES NO

OR

I consider my child/children is not able to give an

informed decision about participation

YES NO 


\section{ASSENT FORM FOR CHILDREN}

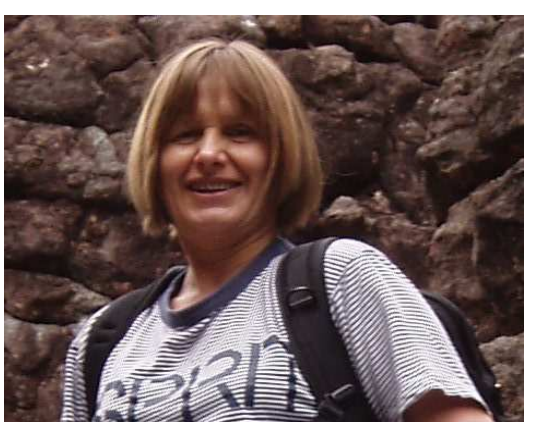

Hi

My name is Alison Stephenson and I am going to be spending some time in your centre doing research.

I am interested in finding out about all the different things you, the other children, and the teachers do while you are at the centre. The ways I am planning to do this are by looking at your portfolio, by watching what teachers and children do, by listening to them, and by talking with the children.

You can choose whether you would like to be part of this research or not by answering each of these questions:

Are you happy that I look at your portfolio? 
Are you happy that I sometimes watch you playing, and YES NO write notes about what you are doing?

Are you happy that I take photographs of you at the centre? (You can have your own copies of the photographs of you)

YES NO

(You can have your own copies of the photographs of you)

Are you happy if I sometimes use a tape recorder to record what is being said?

YES NO

(I use this when everyone talks fast and I find it hard to write all the words down)

Are you happy to talk with me sometimes?

(I will let you choose when you talk to me, so that it doesn't interrupt your playing)

It is fine if you change your mind about what you have said. You just need to tell your family, or the teachers and they will let me know.

I am looking forward to seeing you in the centre

\author{
Alison Stephenson \\ Senior lecturer \\ Wellington College of Education, Victoria University
}




\section{Appendix 7: Data analysis categories and codes}

Initial coding categories were manifest; for example, all data relating to a child were coded to that child, and details of the environment were coded to the physical location and to the play context (e.g. puzzles, quiet room). Other initial categories were broadly analytical; for example, some data were coded 'Null curriculum' reflecting the focus of one research question. As themes emerged, coding became increasingly focused. For example, a cluster of coding was developed around the concept of power, who wielded it, in what circumstances, and to what purpose.

The final and most detailed levels of analysis occurred alongside and as part of the writing process. During this stage as well as revisiting the data as coded, extensive use was made of the Text Search facility of the program. Having the data so easily accessible helped to ensure that the final levels of analysis were firmly grounded in the data.

\section{Activities}

Art outdoors

Art indoors

Balls

Bikes

Books

Carpentry

Clay

Collage

Diggers

Dough

Dramatic

Duplo

Facepaint

Music

Puzzles

Railway

Sand

Swings

Teacher-led activities 
Vehicles

Water

Writing

Adults

Children

\section{Community}

- $\quad$ Adult and child

- Adult and community

- Peer community

○ Anti-adult

- Dramatic themes

○ Historical

○ Older-younger

- Rejection among peers

- Small groups of children

○ Youngest

- Separate adult and child tasks

- Wider community

\section{Curriculum}

- Adult and child tasks

- At a loose end

- Bicultural

- Children's interest

○ Animals/pets

- Being with an adult

○ Birthdays

- Building site

- Building the house

- Dramatic play

- Superhero 
○ Food

○ Horses

○ Listening to stories

○ Making a place

- Media

○ My family

○ Nurturing

○ Physical

- Schema

○ Technology

○ Trains

○ Wild animals

○ Writing

- Children's learning

- At school

- Learning documented

- Discourse of teaching/learning

- Hidden curriculum - teachers

- Individual curriculum

- Mat-times

○ Children's power

○ Quiet times

○ Teacher control

- Meal times

- Peer learning

- Teacher initiated

- Teacher-response activities

- Walks

- Teacher planning

○ Meetings

○ Portfolios

- Toileting

\section{Environment}

- Available to children

○ Closing down

- Baby area 
- Bathroom

- Bicultural

- Changing environment

- Child world/adult world

- Child's perspective

- Distraction and withdrawal

- Dramatic room

- Doors

- Entrance

- Equipment not for children

- Foyer

- Kitchen

- Laundry

- Locker room

- Main room

- Office

- Outside
- Car
- Covered area
○ Deck
○ Fort
○ Garden
○ Playground

- Quiet room

- Sleep room

- Soft environments

- Staff room

- Walls

○ Children's art

- Communication with parents

- Gender

- Media images

- Murals

- Star charts

- Windows 


\section{Families}

- whiteboard notices

\section{Gender}

- boys

- girls

\section{Null curriculum}

- computer

- guns

- dying/death

\section{Planning}

- Notebooks
○ -
○ -
o -

- Six-week planning
○ Aug-Sept
- April - June
○ Feb- April
○ Jan- Feb
- Nov- Dec
○ Sept - Nov

\section{Power}

- Adults power

○ Control strategies

- Acknowledge children's perspective

- Bend the rules

- Concern for injured

- Crowd control

- Direct request

- Discourse of good/wise

- Discourse of teacher I/me

- Distraction 
- Environment

- Indoors and outdoors

- Give reasons

- Give choice

- Ignore minor

- Involve in community

- Negotiate

- Non-verbal communication

- Physical control

- Positive feedback

- Negative feedback

- Question

- Remove child

- Rhymes, songs and games

- Road signs to hold

- Safety

- Teachers support each other

- Threaten

- Within dramatic theme

- Control strategies - why used

- "Appropriate behaviour"

- "Rules"

- Distance disruptive children

- Food

- Involve in community

- Manners

- Obey adult

- Physical wellbeing

- Sleep

- Protect adults

- Protect children from children

- Protect children's play

- Protect environment

- Safety issues

- Tidying 
○ My power as researcher

○ Pressure on teachers

○ Punishments

○ Rules

- Children's power

○ Boys' power

○ Children's use of rules

○ Older-younger

- Power over adults

o With adults

- With me

- Regulations

\section{Research methods}

- Baby book

- Children's names for places

- Favourite activities

- Interviews with parents

- Naughty book

- Photo tour

- Photos - non-tour

- Picture questionnaire

- Portfolios

- Taping

- Why come?

\section{Research role}

- with children

- With families

- With teachers

\section{Rituals}

- Birthdays

- Christmas 
- Family events

- Trips

\section{Routines}

- Mat-times

- Meal times

- Sleeptimes

- Tidying

\section{Teacher role}

- Carer

- Community member - equals

- Controller

- Distracter/guider

- Initiator

- Play companion

- Protector

- Provoker

- Reflector on role

- Responder/appreciator

- Revisiter

- Supervisor

- Supplier

- Supporter

\section{Writing}

